\title{
The use of the latissimus dorsi muscle for cardiac assist
}

Citation for published version (APA):

Lucas, C. M. H. B. (1992). The use of the latissimus dorsi muscle for cardiac assist. [Doctoral Thesis, Maastricht University]. Datawyse / Universitaire Pers Maastricht. https://doi.org/10.26481/dis.19921008cl

Document status and date:

Published: 01/01/1992

DOI:

$10.26481 /$ dis. $19921008 \mathrm{cl}$

Document Version:

Publisher's PDF, also known as Version of record

\section{Please check the document version of this publication:}

- A submitted manuscript is the version of the article upon submission and before peer-review. There can be important differences between the submitted version and the official published version of record.

People interested in the research are advised to contact the author for the final version of the publication, or visit the DOI to the publisher's website.

- The final author version and the galley proof are versions of the publication after peer review.

- The final published version features the final layout of the paper including the volume, issue and page numbers.

Link to publication

\footnotetext{
General rights rights.

- You may freely distribute the URL identifying the publication in the public portal. please follow below link for the End User Agreement:

www.umlib.nl/taverne-license

Take down policy

If you believe that this document breaches copyright please contact us at:

repository@maastrichtuniversity.nl

providing details and we will investigate your claim.
}

Copyright and moral rights for the publications made accessible in the public portal are retained by the authors and/or other copyright owners and it is a condition of accessing publications that users recognise and abide by the legal requirements associated with these

- Users may download and print one copy of any publication from the public portal for the purpose of private study or research.

- You may not further distribute the material or use it for any profit-making activity or commercial gain

If the publication is distributed under the terms of Article $25 \mathrm{fa}$ of the Dutch Copyright Act, indicated by the "Taverne" license above, 
THE USE OF THE LATISSIMUS DORSI MUSCLE FOR CARDIAC ASSIST 
(C) 1992 CMHB Lucas, Maastricht

Productie: Datawyse I Universitaire Pers Maastricht

\section{CIP-GEGEVENS KONINKLIJKE BIBLIOTHEEK, DEN HAAG}

Lucas, Caroline Marie Henriette Bernardine

The use of the latissimus dorsi muscie for cardiac assist I Caroline Marie Henriette Bernardine Lucas. - Maastricht : Universitaire Pers Maastricht. -III.

Proefschrift Maastricht. - Met lit. opg. - Met samenvatting in het Nederlands.

ISBN 90-5278-038-2

NUGI 742,743

Trefw.: cardiomyoplastie / musculus latissimus dorsi / hartchirurgie. 


\title{
THE USE OF THE LATISSIMUS DORSI MUSCLE FOR CARDIAC ASSIST
}

\author{
PROEFSCHRIFT \\ ter verkrijging van de graad van doctor \\ aan de Rijksuniversiteit Limburg te Maastricht, \\ op gezag van de Rector Magnificus, Prof. Mr. M.J. Cohen, \\ volgens het besluit van het College van Dekanen, \\ in het openbaar te verdedigen \\ op donderdag 8 oktober 1992 om 14.00 uur \\ door \\ Caroline Marie Henriette Bernardine Lucas \\ Geboren te Etten-Leur
}

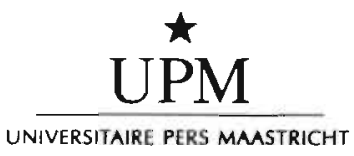




\section{Promotor:}

Prof. Dr. H.J.J. Wellens

\section{Co-promotor:}

Dr. Ir. F.H. van der Veen

Beoordelingscommissie:

Prof. Dr. R.S. Reneman, voorzitter

Prof. Dr. J.W. Arends

Prof. Dr. G. Elzinga

Prof. Dr. W.C. Hülsmann

Prof. Dr. S. Salmons, University of Liverpool, UK

Het in dit proefschrift beschreven onderzoek werd mede mogelijk gemaakt door financiële steun van de Nederlandse Hartstichting.

De uitgave van dit proefschrift werd mede mogelijk gemaakt door financiële steun van: Medtronic Inc., Bakken Research Center, Maastricht; Stichting Wetenschappelijk Durrer-Fonds; Stichting Dr.Ir. J.H.J. van de Laar voor wetenschappelijk biochemisch onderzoek; Stichting RESCAR 
Aan mijn ouders 


\section{CONTENTS}

\section{CHAPTER 1}

Introduction 9

1.1 Historical review 10

1.2 Clinical results of dynamic cardiomyoplasty 16

1.3 The Latissimus Dorsi muscle 18

1.4 Skeletal muscle versus cardiac muscle 18

1.5 Aims of the study 19

\section{CHAPTER 2}

Changes in canine latissimus dorsi muscle during 24 weeks of continuous electrical stimulation $\quad 27$

CHAPTER 3

Adaptations of goat latissimus dorsi muscle using two different protocols of continuous electrical stimulation 45

\section{CHAPTER 4}

Imipramine induced heart failure in the dog: A model to study the effect of cardiac assist devices

\section{CHAPTER 5}

Hemodynamic study following cardiomyoplasty: Comparison of different stimulation protocols 
CHAPTER 6

Long-term follow-up after dynamic cardiomyoplasty in goats 99

\section{CHAPTER 7}

What is the ideal pulse frequency for skeletal muscle stimulation after cardiomyoplasty $\quad 123$

\section{CHAPTER 8}

The importance of muscle relaxation in dynamic cardiomyoplasty 131

\section{CHAPTER 9}

A new stimulation protocol for cardiac assist using the latissimus dorsi muscle 143

Summary 159

Samenvatting 161

Publications 165

Nawoord $\quad 171$

Curriculum Vitae 173 
Chapter 1

\section{INTRODUCTION}

Severe chronic cardiac failure is worldwide an important cause of morbidity and mortality. In the United States alone about 2 million patients suffer from severe pump failure. It is expected that this number will increase in the coming years because of a decrease in mortality from acute myocardial infarction and a growing number of elderly people $(1,2,3)$. Prognosis for patients is poor with a 50\% one-year mortality for patients in New York Heart Association functional class IV (4) with the majority dying suddenly (5).

Pharmaceutical interventions are the major mode of treatment in these patients. In spite of the recently reported beneficial effects of both a combination of hydralazine plus isosorbide dinitrate (6) and angiotensin-converting-enzyme inhibitors $(4,7)$, the chronic nature of the disease with repeated long lasting and costly hospitalizations calls for new therapeutic approaches.

A treatment possibility with good long-term results is cardiac transplantation. However, scarcity of donor hearts and the need for life-long immunosuppressive drugs make this expensive option of limited use.

In recent years a total artificial heart and heart assist devices have been used either for continuous use or as a temporary bridge to transplant $(8,9)$. Unfortunately problems with thromboembolic complications and infection make the long-term use of these devices impossible at the present time.

Not surprisingly therefore, the introduction, during the last decade, of a skeletal muscle, wrapped around the heart and electrically stimulated to contract synchronously with cardiac contraction received considerable attention as a new method to improve cardiac function in patients with severe heart failure. In two different ways one aimed to improve cardiac function; First by adding an extra muscular wall against the myocardium resulting in a decrease in cardiac wall stress. Secondly, to improve cardiac output by electrically stimulating the wrapped muscle resulting in its contraction. In this chapter we will start by reviewing the history of the different ways in which different skeletal muscles have been used for this purpose. 
10

\section{$1.1 \quad$ Historical review}

The idea of using skeletal muscle to assist a failing heart is not new. The use of a variety of skeletal muscles either as free grafts, pedicled grafts or grafts with microvascular anastomoses with or without neural innervation has been investigated. The different muscles include the diaphragm, pectoralis major, rectus abdominis, intercostal, pronator teres, internal oblique, vastus lateralis, sternocleidomastoid, gluteal, sternohyoid, rectus abdominis and the latissimus dorsi (LD). Different ideas were behind the use of these muscles. For example skeletal muscle has been used as a means to revascularize the ischemic myocardium or to enlarge a cardiac chamber to treat congenital defects. Other applications have been the reinforcement of infarcted myocardium or aneurysms. Only more recently, increasing attention has been given to improve cardiac output by electrically stimulating skeletal muscle positioned against or around the heart. Skeletal muscle has also been applied as a tool for counterpulsation by wrapping it around the thoracic or abdominal aorta or by the construction of pouches connected to the systemic circulation functioning as so-called skeletal muscle ventricles.

The first report on the use of skeletal muscle appeared in 1931 when De Jesus described a patient in whom the pectoralis muscle was transplanted to repair a traumatic laceration of the left ventricle (10). Leriche and Fontaine in 1933 investigated the long-term viability of free muscle grafts positioned at the side of an excised part of the left ventricle in dogs (11). In 1935 Beck was the first to describe the transplantation of a pedicled pectoralis major flap into the ischemic myocardium of a patient to relieve anginal symptoms (12). For this purpose diaphragm muscle was used by OSlaughnessy in 1936 (13). In 1938 Griffith and Bates repaired a defect in the right ventricle using a pedicled pectoral major graft (14). In 1946 Weinstein and Shafiroff (15) sutured free muscle grafts from the vastus lateralis muscle and internal oblique muscle around canine hearts resulting in vital muscle parts in two out of six dogs after a follow-up period of 15 weeks.

Petrovsky, starting in 1948, used diaphragm muscle to reinforce aneurysms of the heart in more than 100 patients $(16,17)$.

In 1959 Kantrowitz and McKinnon were the first to apply electrically stimulated skeletal muscle to assist the heart $(18,19)$. They wrapped a pedicled diaphragm graft in one group of dogs around the ventricles and in a second group around the distal aorta. Contraction of the graft was seen on stimulating the phrenic nerve. However. only the wrap around the distal aorta resulted in significant hemodynamic changes as demonstrated by an increase in diastolic pressure when stimulation was performed during cardiac diastole. This started the idea of diastolic counterpulsation using stimulated skeletal muscle.

In 1.964 Nakamura and Glenn transplanted pedicled diaphragm grafts in dogs to enlarge the right atrium and to reinforce the ventricles (20). Both vascularization and innervation was left intact to keep the graft vital. 
Contraction of the graft by stimulating the phrenic nerve was still seen after a follow-up of maximally 9 months. They were the first to demonstrate a temporary positive effect both on right atrial and aortic pressure with the diaphragm muscle wrapped around the ventricles.

In the same year Kusserow and Clapp used electrically stimulated quadriceps femoris muscle for a different purpose. They were able to power a ventricle-type pump successfully during 8 hours, in spite of the fact that some muscle fibre damage occurred (21).

Termet in 1966 was the first to use pedicled latissimus dorsi (LD) muscle flaps sutured onto the ventricles (22). After 8 months of follow-up the majority of the grafts had a normal skeletal muscle structure and contraction of the wrapped muscle at that time resulted in sustained blood pressures of 60 to $80 \mathrm{mmHg}$ during periods of ventricular fibrillation, lasting 15 to 20 minutes.

Shepherd demonstrated the value of paced denervated diaphragmatic pedicled grafts sutured to the right ventricle. He showed that by direct skeletal muscle stimulation vitality of the graft could be preserved (23). Moreover, anastomoses developed between the graft vessels and the coronary circulation.

Another person using diaphragm muscle was Phillips in 1969. In contrast to Nakamura however, he could not obtain hemodynamic improvement with the stimulated graft wrapped around the heart (24).

Kusaba et al applied a diaphragmatic graft to the left ventricle resulting in an acute increase in cardiac output during stimulation of the phrenic nerve synchronous to cardiac contraction. Furthermore they could demonstrate good vitality and intact vascularity of the graft after 4 months of follow-up (25).

In 1978 Thompson used free grafts from canine pronator teres muscle which were applied to the myocardium. During the procedure part of the divided phrenic nerve was implanted into the graft. Six months later all three grafts appeared histologically normal and vital (26).

During the seventies interest also started to assist cardiac function by constructing ventricles from skeletal muscle. Spotnitz et al reported on skeletal muscle ventricles made from canine rectus abdominis muscle (27). Vachon et al used denervated canine diaphragm muscle for this purpose and demonstrated the capability of these pouches to produce pressures of up to $176 \mathrm{mmHg}(28)$. Both groups showed that both muscles functioned according to Starling's law of the heart. Unfortunately, the rectus abdominis muscle appeared to be less compliant compared to cardiac muscle, thus requiring high end-diastolic pressures for optimal performance.

Von Recum also used diaphragm muscle pouches which were able to contract during many hours (29).

Juffe in 1978 used canine gluteus maximus muscle for construction of a counterpulsation pump which was capable to function up to 26 days using 70 electrical stimuli/min during 24 hours a day (30). 


\section{2}

During the last decade the application of skeletal muscle for cardiac assist has benefitted from 2 important new developments. Originally the use of electrically stimulated skeletal muscle was hampered by the inevitably occurrence of muscle fatigue. However, already in 1960 Eccles and Eccles had demonstrated that the physiological and biological properties of skeletal muscle could be changed by cross innervation of two different muscles (31). Then. Salmons and Vrbova made the important discovery that muscle characteristics could be changed by continuous low frequency stimulation (32). Particularly the demonstration of the development of increased fatigueresistance has been of major significance (33). Macoviak showed excellent fatigue-resistance in canine diaphragm muscle which had been stimulated for up to 135 days (34). In 1986 Mannion et al (35) and Acker and coworkers (36) proved that canine LD muscle could be conditioned in such a way that contraction became possible for 24 hours a day.

Another breakthrough was the development of a skeletal muscle stimulator allowing more optimal stimulation of skeletal muscle used for cardiac assistance. By application of a train of pulses instead of single pulse stimulation a tetanic skeletal muscle contraction could be provoked which resembled myocardial contraction $(37,38)$.

During the eighties the possibilities to assist cardiac function with skeletal muscle have been expanded into 3 main directions.

First the replacement of myocardial tissue by stimulated or non-stimulated skeletal muscle with the intention to provide bulk, neovascularization of the myocardium and, when stimulated, new contractile tissue.

Macoviak in 1981 reported about the replacement of a part of the right ventricle by a pedicled diaphragmatic graft which developed tension and muscular thickening on electrical stimulation (39).

Christ and Spira used pedicled non-stimulated LD muscle to reinforce the left ventricular myocardium in dogs, resulting in signs of neovascularization of the heart originating from this skeletal muscle after a maximal follow-up of 1.5 years $(40)$.

To establish revascularization of ischemic myocardium, pectoral muscle with intact neurovascular pedicle was used in dogs by Appiani (41).

Sola, in 1985, used free flaps from the sternocleidomastoid muscle and sternohyoid muscle to replace parts of the left and right ventricle respectively (42).

In the same year Gaines et al published results from the application of a free flap gracilis muscle. revascularized by anastomoses to the right internal mammary vessels, to reconstruct the right ventricular outflow tract. After 10 weeks they found a vital muscle flap with intact blood supply without thrombus formation on the chamber surface (43).

However, full-thickness replacement of myocardial tissue by skeletal muscle resulted in important problems, both in relation to hemostasis between myocardium and the attached skeletal muscle graft and high thrombogenity of the surface of the graft, when used as a new inner lining of a part of the ventricle. To overcome this problem, Dewar and coworkers used a pericar- 
dial patch covered by a LD muscle graft, resulting in early endothelization of this neoendocardium in dogs (44).

In 1987, Cohen et al used a skeletal muscle to repair a myocardial defect in a patient. They successfully closed a full-thickness right ventricular hole with a fascia lata graft reinforced with a rectus abdominis muscle (45).

The second direction has been the application of skeletal muscles to provide effective diastolic counterpulsation.

Neilson et al developed assist devices constructed from rectus abdominis muscle and LD muscle, which contracted against a bladder connected to the aorta or to a conduit between the left ventricle and the aorta. In acute experiments they could show effective counterpulsation (46).

Stevens et al also constructed a counterpulsation device, consisting of a double open-ended compressible pouch, wrapped with canine rectus abdominis muscle, and interpositioned between the left ventricle and the aorta. Effective diastolic pressure augmentation and increase in cardiac output was obtained in acute experiments (47).

A large number of experiments using skeletal muscle in counterpulsation devices have been performed by Chiu et al and Stephenson and coworkers. Chiu's group used canine LD muscle in situ. A balloon was inserted beneath this muscle which was subsequently connected to the aorta with a dacron graft. The main advantages of this technique were the absence of extensive dissection of the LD muscle, needed for this device, and the possibility to use the original stretch of the LD muscle to transport power to the balloon. In this way the risk of loosing part of the power after distal dissection of the muscle could be minimized. They demonstrated that effective diastolic pressure augmentation could be produced with canine LD muscles, which were preconditioned for 8 to 18 weeks (48). However, long-term thromboembolic sequelae due to the presence of blood inside the balloon forced them to look for different solutions. This led to the construction of a dual-chamber extra-aortic counterpulsation system consisting of a hydraulic bulb, positioned beneath the left LD muscle in dogs. The bulb was connected to a graft positioned parallel to the aorta. The entire device was filled with fluid. Compression of the bulb by the stimulated LD muscle resulted in fluid displacement within the system, leading to narrowing of two membranes within the graft and elevation of diastolic pressure (49). Optimal timing of the skeletal muscle contraction during diastole was accomplished by the development of a new pacemaker, which automatically adapted its pacing delay to changes in heart rate (50).

A large series of experiments has been performed by the group of Stephenson, using so-called skeletal muscle ventricles (SMV). These ventricles consist of a pouch constructed from LD muscle wrapped in a multilayered conical fashion. During their first experiments. using such a device, these investigators could demonstrate the capability of these ventricles to produce flow during 4 hours, using a hydraulic test system (51). Acute studies, done with SMVS constructed from preconditioned LD muscles, showed that stroke work of the device was intermediate between that of the dog's left and 
right ventricle (52). Subsequent studies showed that a vascular delay period between SMV construction and stimulation of the LD muscle and also conditioning of the LD muscle by electrical stimulation had a positive effect on the output produced by these ventricles (53). In 1986, it became possible to obtain effective diastolic counterpulsation for 1 hour after connecting the SMV to the aorta in a dog (53). The next step in their investigations was the construction of a totally implantable mock circulation to which the SMV was connected. That device allowed the separate regulation of pre- and afterload and long-term pressure and flow measurements. They could demonstrate substantial flow and pressure build-up after maximally 9 weeks of continuous pumping underlining the capability of the LD muscles to perform long-term work (54).

In 1987, they showed that LD muscles also could be conditioned to perform continuous work during their functioning as SMV's, indicating that in situ preconditioning of the LD muscle was not necessary (55). In the same year they reported about the ability of the SMVS to function in the systemic circulation as diastolic counterpulsators during maximally 11 weeks (56). However, disadvantages of this application became apparent like aortic dissection at the site of the connection and renal infarction following thromboembolic episodes. To prevent thromboembolic complications a new experimental device was tested by Anderson et al (57), using a SMV made from wrapped LD which was connected to a pump device inserted into the thoracic aorta. This device consisted of two shells. Air could be compressed in between those shells by the contracting LD muscle leading to displacement of blood within the aorta. This construction prevented the stasis of blood. Diastolic counterpulsation was achieved in dogs up to 42 days after the start of skeletal muscle stimulation. Main cause of death after this period was disruption of the aorta. Unfortunately, thromboemboli were still seen in the spleen and kidneys in 2 of the 4 dogs.

During the last years much attention has been given to further improvement of the functional properties of the SMV's. In 1989, Bridges and coworkers (58) constructed a SMV which was capable to function optimally at physiological preloads from 5 to $15 \mathrm{mmHg}$ in contrast to the higher preloads used previously. Also the effect of different ways of LD preconditioning and the application of stretch to the muscle was tested. In this way the maximal attained SMV stroke work amounted to $59 \%$ of the canine left ventricular stroke work. The same group also showed that SMV, made from preconditioned canine LD muscles, were capable to perform the work of the right ventricle with physiological filling pressures (59). Pochettino et al demonstrated that SMV's, 18 weeks after their construction from unconditioned LD muscles, could produce $194 \%$ of left ventricular stroke work (60). After a period of LD electrical preconditioning. this decreased to 54\%. Their experiments stressed the need to allow the wrapped LD muscle an adequate vascular delay period between wrapping and electrical stimulation.

Recently, Anderson and coworkers (61) reported on their long-term experience with LD SMV's functioning in the systemic circulation. The survival period in 10 dogs ranged from 1 week to 9 months with all animals 
demonsirating effective diastolic counterpulsation. However, only 3 dogs remained free from thrombus formation in the SMV, all 3 having a pleural lining of their SMV cavity. This suggests that autogenous lining of the inner cavity of the SMV's might be the solution to prevent thromboembolic complications as a major obstacle, preventing widespread use of SMVs.

It has also been shown recently that adequate stroke work can be maintained in SMVS made from conditioned LD muscles, even in the presence of scvere hypotension during heart failure (61).

In 1990 a different method applying skeletal muscle for diastolic counterpulsation was described by Chachques et al (62). In goats, they wrapped the right $\mathrm{LD}$ muscle around the ascending aorta with or without enlargement by a pericardial patch. Effective diastolic counterpulsation was obtained, both before and after the induction of heart failure.

So far, the counterpulsation techniques mentioned have not been applied in patients because problems remain to be solved in particular in relation to thromboembolic complications.

The third main area of research using skeletal muscle for cardiac assist has been the concept of dynamic cardiomyoplasty. During that procedure, a skeletal muscle is wrapped around the heart and electrically stimulated synchronously with cardiac contraction. In most cases the latissimus dorsi muscle is used for this purpose. Pioneers in this field have been Carpentier and Chachques. In 1985 their first report about animal research was published using a canine LD flap wrapped around the heart (63). After 3 to 6 months follow-up without stimulation the LD muscle appeared histologically well preserved and capable to increase cardiac output for 2 hours when stimulated acutely. This article was very soon followed by the publication of the first clinical case (64). The left LD muscle was wrapped around the heart in a female patient, to replace and reinforce a part of the ventricle. after resection of a large cardiac tumor. Since then several articles have been published by this group, reporting on experimental data from goats and clinical results in patients (65-67).

Important observations have also been made by the group of Jatene in South America $(68,69)$. In that part of the world, use of this technique has been promoted by the presence of a large group of relatively young patients suffering from dilated cardiomyopathy due to Chagas disease. Jatene et al published on the results of long-term in situ conditioning of canine LD muscle (68) and the effect on cardiac output when the stimulated preconditioned LD muscle was wrapped around the heart (69).

In the United States of America both Stephenson et al and Magovern and coworkers investigated the use of the LD muscle for dynamic cardin. myoplasty. Stephenson et al reported disappointing long-term results from this technique due to deterioration of the LD muscle (70). Results from the group of Magovern were more promising demonstrating in dogs an increase in cardiac output due to the contracting wrapped LD muscle after the induction of cardiac failure (71). Unfortunately, no histological data from these wrapped LD muscles were supplied.

Soberman et al described that the electrically stimulated canine LD muscle 


\section{6}

wrapped around the right ventricle resulted in increased right ventricular pressure and ejection fraction (72).

Lee et al investigated the effect of dynamic cardiomyoplasty in dilated canine hearts during acute experiments (73). They demonstrated augmentation of cardiac performance and a lowered myocardial oxygen consumption.

\section{2}

\section{Clinical results of dynamic cardiomyoplasty}

In contrast to SMV's, which have not been used clinically, dynamic cardiomyoplasty has been applied in over 100 patients (74). At the present time several groups throughout Europe, South America, the United States and Canada are investigating the usefulness of the dynamic cardiomyoplasty procedure to treat severe heart failure in patients, as shown by the communications during several meetings which were held during the last years (75-77).

Published clinical data have been controversial. Some centers described significant increases in cardiac function, as evaluated by a large variety of methods like invasive hemodynamic measurements, the application of both M-mode echo-evaluation, echo-Doppler flow measurements and nuclear techniques (78-80). Whereas left ventricular ejection fraction showed a significant increase in only the minority of cases most clinical data showed significant improvement in functional class of the New York Heart Association (81-83). Follow-up of a group of 74 patients was summarized by Grandjean in 1991 (74). From this series 27 patients with a follow-up of more than 6 months showed a mean increase in left ventricular ejection fraction of $3.7 \%$. Eightyfive percent of these patients demonstrated a significant improvement in New York Heart Association functional class.

Evaluation of the effectiveness of dynamic cardiomyoplasty in patients is difficult for a number of reasons.

First; the methods to evaluate the cardiac and functional condition of the patients are not standardized.

Second; in a number of centers concomitant surgery was performed like aneurysm or tumor resection, valve replacement and/or coronary bypass grafting. thereby obscuring the effect of dynamic cardiomyoplasty alone. Only Jatene reported follow-up data of patients with dilated cardiomyopathy treated solely by dynamic cardiomyoplasty (78).

Third; the clinical condition of the patients before operation varied. Until now no strict rules have been given for selection of patients for this procedure. Clinical results indicate that patients in severe cardiac failure (New York Heart Association functional class IV) have a large peri-operative mortality $(79,81,74)$. Moreover, improvement in cardiac function has been less in patients with a very large left ventricular end-diastolic diameter, probably because the LD muscle can not be completely wrapped around the heart. Recently Magovern and coworkers reported that the optimal longterm outcome depended in particular on the right ventricular ejection fraction, which had to be above 30\% (84). 
How to explain subjective improvement in a number of patients with only a small or even no increase in cardiac output.

First of all, a placebo effect can not be excluded. Secondly, it has been reported that adding an extra muscular wall by the $\mathrm{LD}$ muscle leads to a decrease in both systolic and diastolic wall stress with a concomitant decrease in myocardial oxygen consumption (73). This may lead to partial recovery of myocardial function $(78,85)$ or even to in future reversal of the remodeling, which the myocardium undergoes during the progressive course of chronic congestive heart failure (86-88). Moreover, it has been described that increased wall thickness relative to ventricular volume is related to increased survival in patients with cardiomyopathy (89). Also, recovery of myocardial function because of the development of collateral circulation from the LD muscle to the myocardium has to be kept in mind. Thirdly, by preventing further dilatation of the heart the wrapped LD muscle could halt or delay the progressive downward spiraling clinical course which these patients often experience (90). Both this point and the possible development of collateral circulation from the wrapped muscle could be responsible for the decrease in ventricular arrhythmias which has been described in patients after dynamic cardiomyoplasty (91). Fourthly, it could be that in particular improvement of right ventricular function is important in the increase in exercise tolerance in a number of these patients. As already mentioned, Baker et al (92) and Franciosa and coworkers (93) reported that maximal oxygen consumption and exercise tolerance was correlated with right ventricular ejection fraction, rather than left ventricular ejection fraction.

Fifth, an indirect effect of dynamic cardiomyoplasty on skeletal muscle can not be excluded. As described by Sullivan and coworkers, chronic congestive heart failure leads to abnormalities in peripheral vasculature and skeletal muscle fibers (94-96). Exercise training in these patients has a significant positive effect on exercise tolerance $(97,98)$. Such improvement can be seen in patients after coronary artery bypass grafting, even in the absence of a formal training programme following the operation (99). It could be that the increased confidence and motivation in patients after a cardiomyoplasty procedure to exercise more, either or not guided by an exercise training programme, does contribute to the increased exercise tolerance and improved well-being.

\section{3}

\section{The Latissimus Dorsi muscle}

In most cases the latissimus dorsi muscle is chosen for application in a dynamic cardiomyoplasty procedure. Use of this muscle has important advantages. First of all, it has a mass which is comparable to the left ventricle. It can easily be mobilized and is located not too far from the heart, making it possible to transpose a large part of the muscle into the thoracic cavity around the heart. Moreover, the main part of the muscle is connected 


\section{8}

to a large neurovascular pedicle, containing the thoracodorsal artery, vein and nerve $(100,101)$. Also, a clean dissection of this muscle from its original location is possible, without major damage to the muscle.

\section{4}

\section{Skeletal Muscle versus Cardiac Muscle}

A key question, when investigating the use of skeletal muscle to assist the heart. inevitably is how much of the function of the specialized myocardial muscle fibers can be taken over by skeletal muscle fibers.

Considering this question. how do skeletal and cardiac muscle compare to each other (102-104)?

Both are striated muscle, composed of muscle cells with longitudinally oriented myofibrils which are organized in sarcomeres. The mechanism of contraction of the individual cells of both muscle types is basically the same and based upon the shortening of sarcomeres by the sliding-filament theory, due to the binding of $\mathrm{Ca}^{2+}$ to troponin. Also the process of relaxation is comparable. resulting from the active transport of $\mathrm{Ca}^{2+}$ from the cytoplasm into the sarcoplasmic reticulum depending upon the activity of Ca-ATPase. However, there are important differences both structurally and functionally. Cardiac muscle cells are branched cells (maximally $100 \mu \mathrm{m}$ in length) with one central nucleus and an abundance of mitochondria. This is needed because the heart has to perform aerobic work for 24 hours a day, without creating an oxygen debt. Skcletal muscle cells are long with variable length. sometimes running along the entire length of the muscle. They have a considerably smaller amount of mitochondrial volume percentage because no continuous activity is required and a deficit of oxygen can be dealt with for a short period of time. Also important differences exist between the mode of excitation of both types of muscle. Firstly, the heart has its own pacemaker in contrast to skeletal muscles. Excitation of cardiac muscle cells results from a depolarization wave spreading from the pacemaker site (normally the sinus node) to the myocardial muscle cells by way of a specialized conduction system and, more distally, along the intercalated discs which connect the individual muscle cells. In this way the heart act as a functional syncytium and contracts in an all-or-none fashion. In contrast. skeletal muscle cells, organized in motor-units, are separately innervated by individual nerve branches. Important differences exist in the way excitationcontraction coupling is organized. In cardiac muscle, apart from $\mathrm{Ca}^{2+}$ originating from the sarcoplasmic reticulum, extracellular $\mathrm{Ca}^{2+}$ is necessary for coupling. In contrast, skeletal muscle cells can rely on their sarcoplasmic reticulum as a source for the $\mathrm{Ca}^{2+}$, needed for the subsequent crossbridge cycling. Also, gradation of force is organized in a different way. As mentioned, the entire myocardium is activated in an all-or-none fashion. Myocardial contractile force is mediated both by the number of activated actin-myosin crossbridges per sarcomere and the degree of activation of the individual muscle fibers. Availability and utilization of $\mathrm{Ca}^{2+}$ play a central role in this process. Skeletal muscle force, however, is regulated by activa- 
tion of a larger or smaller number of motorunits (spatial summation) and by increasing the number of consecutive depolarizations of the muscle cell membrane, resulting in a tetanic contraction (temporal summation). Finally, different skeletal muscle fibre types exist in contrast to a single type of cardiac muscle fibers. Two main skeletal muscle fibre types have been described with several intermediate forms. Slow-twitch type I fibers, having a large number of mitochondria, which are loaded with an extensive amount. of aerobic enzymes, thus enabling contractile activity of long duration. This is in contrast to the fast-twitch type II fibers, with a smaller mitochondrial volume and a high activity of anaerobic enzymes to perform fast forceful contractions of short duration.

These differences between cardiac and skeletal muscle fibers do have important implications when skeletal muscle is used to assist the heart.

Firstly, skeletal muscle has to be able to function continuously during 24 hours a day. Therefore a number of adaptations within the skeletal muscle fibers have to take place to make this possible. Buller et al in 1960 (105) and Salmons and Vrbova in 1969 (32) described the impressive capacity of skeletal muscle to adapt itself to changing activation patterns. However, are these adaptations sufficient to assist a failing heart full-time?

Secondly, a skeletal muscle does not have a pacemaker. Stimulation has to be performed by an external puIse stimulator. This raises the question about the optimal way of stimulating the LD muscle both when electrical stimulation is started and after the muscle has been stimulated for several weeks

In this thesis, our experience with dynamic cardiomyoplasty in dogs and goats is presented. The study was done first of all to investigate if a skeletal muscle (LD) can be conditioned to produce tetanic burst contractions during 24 hours a day. Both dogs and goats were used for this purpose.

A second problem, addressed in this thesis, is the optimal way of stimulation of both unconditioned and conditioned LD muscle.

The most important question to answer was: "Is the LD muscle, when wrapped around the heart able to improve normal and depressed cardiac function?". We studied this both immediately after wrapping and after a prolonged period with continuous electrical stimulation following wrapping.

The content of the different chapters is as follows:

Chapter 2 discusses functional, structural, histological and biochemical changes which occur during long-term in situ stimulation of the canine LD muscle.

The same features are investigated in the in situ stimulated goat LD muscle (Chapter 3).

To evaluate the usefulness of dynamic cardiomyoplasty, it is essential to test the effect of this surgical technique in a failing heart model. We used 
imipramine to create a safe, reproducible and reversible failing heart model (Chapter 4).

In Chapter 5 the acute effects of dynamic cardiomyoplasty, both in the normal and depressed goat heart, are shown using different modes of electrical stimulation of the wrapped LD muscle.

Chapter 6 gives the long-term hemodynamic and histologic results of dynamic cardiomyoplasty in a large series of goats.

The functional characteristics of the conditioned LD muscle in dogs using different modes of electrical stimulation are presented in Chapter 7.

Chapter 8 presents data indicating that the currently used clinical stimulation protocol might be hazardous for the wrapped LD muscle in the end. This is done by presenting both goat and human data.

In view of the observations described in Chapter 8 , an alternative stimulation protocol is suggested and the functional and histological data of that protocol are presented in Chapter 8.

The thesis closes with a summary in the English and Dutch language. 


\section{REFERENCES}

1. Yusuf S. Thom T, Abbott RD: Changes in hypertension treatment and in congestive heart failure mortality in the United States. Hypertension 1989: 13(suppl I):74-79.

2. Franciosa JA: Epidemiologic patterns, clinical evaluation, and long-term prognosis in chronic congestive heart failure. Am J Med 1986;80(suppl 2B): 14-21.

3. De Vreede JJM, Gorgels A, Verstraaten G, Vermeer F, Dassen WRM, Wellens HJJ: Did prognosis after acute myocardial infarction change during the past 30 years? A meta-analysis. JACC 1991;18:698-706.

4. The CONSENSUS Trial Study Group: Effects of enalapril on mortality in severe congestive heart fallure: results of the Cooperative North Scandinavian Enalapril Survival Study (CONSENSUS). N Engl J Med 1987;316:1429-1435.

5. Franciosa JA, Wilen M, Ziesche S, Cohn JN: Survival in men with severe chronic left ventricular failure due to either coronary heart disease or idiopathic dilated cardiomyopathy. Am J Cardiol 1983:51:831-836.

6. Cohn JN, Archibald DG, Phil M, Ziesche S, Franciosa JA, Harston WE. Tristani FE, Dunkman WB, Jacobs W, Francis GS, Flohr KH, Goldman S, Cobb RF. Shah PM, Saunders R, Fletcher RD, Loeb HS. Hughes VC, Baker B: Effect of vasodilator therapy on mortality in chronic congestive heart failure. Results of a Veterans Administration Cooperative Study. N Engl J Med 1986;314:1547-1552.

7. The SOLVD Investigators: Effect of enalapril on survival in patients with reduced left ventricular ejection fractions and congestive heart failure. $N$ Engl $J$ Med 1991:325:293-302.

8. Davis PK, Rosenberg G, Snyder AJ, Pierce WS: Current status of permanent total artificial heart. Ann Thorac Surg 1989:47:172-178.

9. Hill JD: Bridging to cardiac transplantation. Ann Thorac Surg 1989:47:167-171.

10. De Jesus JR: Breve concideraciones sobre un case de herida penetrante del corazon. Bol Asoc Med (Puerto Rico) 1931;23:380-382.

11. Leriche R. Fontaine R: Essai experimental de traitement de certains, infaretus du myocarde et de l'aneurisme du coeur par une greffe de muscle strie. Bull Soc Nat Chir 1933;59:229-232.

12. Beck CS: The development of a new blood supply to the heart by operation. Ann Surg 1935;102:801-807

13. O'Slaughnessy L: An experimental method of providing a collateral circulation to the heart. Br J Surg 1936:23:665-669.

14. Griffith GC. Bates W: A ventricular perforation in transplanting a new blood supply. New Internat Clinics NS 1938:2:17-20.

15. Weinstein M. Shafiroff BG: Grafts of free muscle transplants upon the myocardium. Science 1946; 104(2705):410-412.

16. Petrovsky BV: The use of diaphragm grafts for plastic operations in thoracic surgery. J Thorac Cardiovasc Surg 1961:41:348-355.

17. Petrovsky BV: Surgical treatment of cardiac aneurysms. J Cardiovasc Surg (Torino) 1966:2:87-91.

18. Kantrowitz A, McKinnon W: The experimental use of the diaphragm as an auxiliary myocardium. Surg Forum 1959;9:266-268.

19. Kantrowitz A: Functioning autogenous muscle used experimentally as an auxiliary ventricle. Trans Am Soc Artif Int. Organs 1960;6:305-310.

20. Nakamura K, Glenn WL: Grafts of diaphragm as a functioning substitute for the myocardium. J Surg Res 1964:4:435-439. 


\section{2}

21. Kusserow BK, Clapp III JF: A small ventricle-type pump for prolonged perfusions: Construction and initial studies, including attempts to power a pump biologically with skeletal muscle. Trans Amer Soc Artif Int Organs 1964:10:74-78.

22. Termet H, Chalencon JL, Estour E, Gaillard P, Favre JP: Transplantation sur le myocarde d'un muscle strie par excite par pacemaker. Ann Chir Thorac Cardio 1966:5:568-571.

23. Shepherd MP: Diaphragmatic muscle and cardiac surgery. Ann R Coll Surg Engl 1969;45:212-231.

24. Phillips WL, Pallin S. Crastnopol P: Diaphragm transplantation. Angiol 1969; 20:635-640.

25. Kusaba E, Schraut W, Sawatani S, Javon D, Freed P, Kantrowitz A: A diaphragmatic graft for augmenting left ventricular function: a feasibility study. Trans Am Soc Artif Int Organs 1973; 19:251-257.

26. Thompson N: Preliminary report on experimental free autografts of skeletal muscle to the myocardium. Scand J Plast Reconstr Surg 1978;12:189-193.

27. Spotnitz HM. Merker C. Malm JR: Applied physiology of the canine rectus abdominis: Force-length curves correlated with functional characteristics of a rectus powered "ventricle". Potential for cardiac assistance. Trans Am Soc Artif Int Organs 1974:20:747-756.

28. Vachon BR, Kunov H: Mechanical properties of diaphragm muscle in dogs. Med Biol Eng Comput 1975;13:252-260.

29. Von Recum A. Stule JP. Hamada O: Long-term stimulation of a diaphragm muscle pouch. Surg Res 1977;23:422-427.

30. Juffe A, Ricoy. JR, Marquez J, Castillo-Olivares JL. Figuera D: Cardialization: a new source of energy for circulatory assistance. Vasc Surg 1978:12:10-17.

31. Eccles JC. Eccles RM: Interaction between motor neurons and muscles in respect of the characteristic speeds of their responses. J Physiol Lond 1960;150:417439.

32. Salmons S. Vrbova G: The influence of activity on some contractile characteristics of mammalian fast and slow muscles. J Physiol Lond 1969:210:429439.

33. Salmons S. Henriksson J: The adaptive response of skeletal muscle to increased use. Muscle \& Nerve 1981:4:94-105.

34. Macoviak JA. Stephenson LW. Armenti F, Kelly AM, Alavi A, Mackler T, Cox J, Palatianos G. Edmunds LH: Electrical conditioning of in situ skeletal muscle for replacement of myocardium. J Surg Res 1982:32:429-439.

35. Mannion JD, Bitto T, Hammond RL, Rubinstein NA. Stephenson LW: Histochemical and fatigue characteristics of conditioned canine latissimus dorsi muscle. Circ Res 1986;91:534-544.

36. Acker MA, Hammond RL. Mannion JD, Salmons S, Stephenson LW: An autologous biologic pump motor. J Thorac Cardiovasc Surg 1986:92:733-746.

37. Drinkwater DC Jr, Chiu RC-J. Modry DL. Wittndch C: Cardiac assist and myocardial repair with synchronously stimulated skeletal muscle. Surg Forum 1980:31:271-274.

38. Dewar ML, Drinkwater DC, Wittnich C, Chiu RC-J: Synchronously stimulated skeletal muscle graft for myocardial repair. J Thorac Cardiovasc Surg 1984; 87:325-331.

39. Macoviak JA. Stephenson LW. Spielman S, Greenspan A. Likoff M, St John Sutton M. Reichek N. Rashkind W. Edmunds Jr LH: Replacement of ventricular myocardium with diaphragmatic skeletal muscle. Short-term studies. J Thorac Cardiovasc Surg 1981:81:519-527. 
40. Christ JE, Spira M: Application of the latissimus dorsi muscle to the heart. Ann Plast Surg 1982;8:118-121.

41. Appiani E, Almada H, Garlando C, Milei J, Bastardi J: Potential myocardial revascularization and mechanical effects of pectoral muscle transplants. Ann Plast Surg 1983:10:252-263.

42. Sola AM, Dallard DH, Ivey TD, Haneda K, Itoh T. Thomas R: Autotransplantation of skeletal muscle into myocardium. Circulation 1985;71:341-348.

43. Gaines WE. Goldberg NH. Mergner WJ. Tommaso CL, Moulton AL, McLaughlin JS: Reconstruction of the right ventricular outflow tract with a vascularized free flap of striated muscle. Surg Forum 1985;36:250-252.

44. Dewar M. Walsh G, Abraham A, DeSimon J, Foot E. Stewart DVMJ, Fraser R, Chiu RC-J: Left ventricular full-thickness cardiomyoplasty with pericardial neoendocardium: Experimental development of a surgical procedure. Ann Thorac Surg 1987:44:618-624.

45. Cohen M. Marshall MA, Goldfaden DM, Silverman NA: Repair of right ventricular free wall defect with a pedicled muscle flap. Ann Thorac Surg 1987:44:651-652.

46. Neilson IR, Brister SJ. Khalafalla AS: Left ventricular assistance in dogs using a skeletal muscle powered device for diastolic augmentation. Heart Transplant $1985 ; 4: 343-347$

47. Stevens L. Badylak SF. Janas W. Gray W, Geddes LA, Voorhees III WD: A skeletal muscle ventricle made from rectus abdominis muscle in the dog. J Surg Res 1989:46;84-89.

48. Chiu RC-J. Walsh GJ, Dewar ML, DeSimon J-H, Khalafalla AS, Ianuzzo D: Implantable extra-aortic balloon assist powered by transformed, fatigue-resistant skeletal muscle. J Thorac Cardiovasc Surg 1987:94:694-701.

49. Kochamba G. Desrosiers C, Dewar M, Chiu RC J: The muscle-powered dual chamber counterpulsator: Rheologically superior implantable cardiac assist device. Ann Thorac Surg 1988;45:620-625.

50. Li CM. Hill A. Colson M. Desrosiers C, Chiu RC-J: Implantable rate-responsive counterpulsation assist system. Ann Thorac Surg 1990:49:356-362.

51. Mannion JD. Acker MA, Hammond RL: Four-hour circulatory assistance with canine skeletal muscle ventricles. Surg Forum 1986;37:211-213.

52. Mannion JD. Acker MA. Hammond RL. Faltemeyer W. Duckett S. Stephenson LW: Power output of skeletal muscle ventricles in circulation: short-term studies. Circulation 1987;76:155-162.

53. Mannion JD. Hammond RL, Stephenson LW: Hydraulic pouches of canine latissimus dorsi: Potential for left ventricular assistance. J Thorac Cardiovasc Surg 1986:91:534-544.

54. Acker MA. Hammond RL. Mannion JD, Salmons S. Stephenson LW: An autologous biologic pump motor. J Thorac Cardiovasc Surg 1986:92:733-746.

55. Acker MA. Hammond RL. Mannion JD. Salmons S. Stephenson LW: Skeletal muscle as the potential power source for a cardiovascular pump: Assessment in vivo. Science 1987:236:324-327.

56. Acker MA. Anderson WA. Hammond RL, Chin AJ, Buchanan JW, Morse CC, Kelly AM. Stephenson LW: Skeletal muscle ventricles in circulation: One to eleven weeks' experience. J Thorac Cardiovasc Surg 1987;94:163-174.

57. Anderson WA, Bridges CR, Chin AJ. Andersen JS. Acker MA. Hammond RL, Dimeo F, Calahan PT, Gale DR, Brown WE, Stephenson LW: Long-term neurostimulation of skeletal muscle: Its potential for a tether-free biologic cardiac assist device. PACE 1988:11:2128-2134. 


\section{4}

58. Bridges CR, Brown WE, Hammond RL. Anderson DR, Anderson WA. Dimeo F, Stephenson LW: Skeletal muscle ventricles: Improved performance at physiologic preloads. Surgery 1989; 106:275-282.

59. Bridges CR. Hammond RL, Dimeo F, Anderson WA, Stephenson LW: Functional right-heart replacement with skeletal muscle ventricles. Circulation 1989 (suppl III):80: $183-191$.

60. Pochettino A, Spanta AD. Hammond RL, Anderson DR, Bridges CR, Samet $P$, Ninami H, Hohenhaus E. Salmons S. Stephenson LW: Skeletal muscle ventricles for total heart replacement. Ann Surg 1990;212:345-352.

61. Anderson DR. Pochettino A, Hammond RL. Hohenhaus E, Spanta AD, Bridges CR, Lavine S. Bhan RJ. Colson M. Stephenson LW: Autogenously lined skeletal muscle ventricles in circulation: Up to nine months' experience. J Thorac Cardiovasc Surg 1991; 101:661-670.

61. Pochettino A. Anderson DR. Hammond RL, Salmons S. Stephenson LW: Skeletal muscle ventricles. Sem Thorac Cardiovasc Surg 1991:3:154-159.

62. Chachques JC, Grandjean PA, Cabrera Fischer EI, Latremouille C, Jebara VA, Bourgeois I, Carpentier A: Dynamic cardiomyoplasty to assist left ventricular failure. Ann Thorac Surg 1990:49:225-230.

63. Chachques JC, Mitz V. Hero M. Arhan P, Gallix P. Fontaliran F, Vilain R: Experimental cardioplasty using the latissimus dorsi muscle flap. J Cardiovasc Surg 1985;26:457-462.

64. Carpentier A. Chachques JC: Myocardial substitution with a stimulated skeletal muscle: First successful clinical case. Lancet 1985;1:1267.

65. Chachques JC. Grandjean P. Schwartz K. Mihaileanu S. Fardeau M, Swynghedauw B. Fontaliran F, Romero N, Wisnewsky C, Perier P, Chauvaud S, Bourgeois I, Carpentier A: Effect of Latissimus dorsi dynamic cardiomyoplasty on ventricular function. Circulation 1988:78(suppl III);203-216.

66. Carpentier A. Chachques JC: Latissimus dorsi cardiomyoplasty to increase cardiac output. In: Rabago G, Cooley A (Eds.). Heart valve replacement: Current status and future trends. Futura Publishing Company Inc., Mount Kisco. NY. 1987:pg473-486.

67. Chachques JC. Grandjean PA. Carpentier A: Latissimus dorsi dynamic cardiomyoplasty. Ann Thorac Surg 1989;47:600-604.

68. Leirner AA. Moreira LFP, Chagas ACP. Cestari LA. Oshiro MS, Nakayama E, da Luz PL, Stolf NAG, Jatene AD: Biomechanical circulatory assistance. Importance of aerobic capacity of normal and conditioned skeletal muscles. Trans Am Soc Artif Int Organs 1988;34:716-720.

69. Chagas ACP. Moreira LFP. da Luz PL, Camarano GP, Leirner A, Stolf NAG, Jatene AD: Stimulated preconditioned skeletal muscle cardiomyoplasty. An effective means of cardiac assist. Circulation 1989(suppl III);80:202-208.

70. Anderson WA, Andersen JS, Acker MA, Hammond RL, Chin AJ, Douglas PS, Khalafalla AS. Salmons S, Stephenson LW: Skeletal muscle grafts applied to the heart. A word of caution. Circulation 1988;78(suppl III);180-190.

71. Kao RL. Christlteb IY, Magovern GJ, Park SB, Magovern Jr GJ: The importance of skeletal muscle fiber orientation for dynamic cardiomyoplasty. J Thorac cardiovasc Surg 1990:99:134-140.

72. Soberman MS, Wornom III IL, Justicz AG, Coleman III JJ, Austin GE, Alazraki NP. Sink JD: Latissimus dorsi dynamic cardiomyoplasty of the right ventricle. Potential use as a partial myocardial substitute. $J$ Thorac Cardiovasc Surg 1990:99:817-827. 
73. Lee KF, Dignan RJ. Parmar JM, Dyke CM, Benton G, Yeh T. Abd-Eilfattah AS, Wechsler AS: Effects of dynamic cardiomyoplasty on left ventricular peiformance and myocardial mechanics in dilated cardiomyopathy. J Thorac Cardiovasc Surg 1991;102:124-131.

74. Grandjean PA, Austin L, Chan S. Terpstra B. Bourgeois IM: Dynamic cardiomyoplasty: Clinical follow-up results. J Cardiac Surg 1991;6:80-88.

75. Chiu RC-J, Bourgeois IM (Eds.): Transformed muscle for cardiac assist and repair. Futura Publishing Company Inc., Mount Kisco, NY, 1990.

76. Carpentier A, Chachques JC, Grandjean P (Eds.): Cardiomyoplasty. Futura Publishing Company Inc. Mount Kisco, NY, 1991.

77. Stephenson LW (Ed.): J Cardiac Surgery 1991;6 (suppl).

78. Jatene AD, Moreira LFP, Stolf NAG, Bocchi EA, Seferian P. Fernandes PMP, Abensur $H$ : Left ventricular function changes after cardiomyoplasty in patients with dilated cardiomyopathy. J Thorac Cardiovasc Surg 1991;102:132-139.

79. Carpentier A, Chachques JC: Clinical dynamic cardiomyoplasty: Method and outcome. Sem Thorac Cardiovasc Surg 1991:3:136-139.

80. Almada H. Molteni L, Ferreira R, Ortega D, Cianciulli T, Llanos S, Prezioso H: The value of the echo-Doppler in cardiomyoplasty procedures. J Cardiac Surg 1991: 6(suppl): 113-118.

81. Magovern JA. Furnary AP, Christlieb IY, Kao RL, Park SB, Magovern GJ: Indications and risk analysis for clinical cardiomyoplasty. Sem Thorac Cardiovasc Surg 1991;3:145-148.

82. Delahaye F, Jegaden O, Montagna P, Desseigne P, Blanc P, Vedrinne C. Touboul P. Saint-Pierre A. Perinetti M. Rossi R, Itti R, Mikaeloff P: Latissimus dorsi cardiomyoplasty in severe congestive heart failure: The Lyon experience. $J$ Cardiac Surg 1991;6(suppl): 106-112.

83. Magovern GJ, Christlieb IY, Kao RL: The Allegheny Hospital experience. In: Carpentier A. Chachques JC, Grandjean P (Eds.). Cardiomyoplasty. Futura Publishing Company Inc., Mount Kisco, NY, 1990;159-170.

84. Magovem JA, Christlieb IY, Orie JE: Early results of cardiomyoplasty. Circulation 1990;82 (suppl III):383.

85. Moreira LFP, Stolf NAG, Jatene AD: Benefits of cardiomyoplasty for dilated cardiomyopathy. Sem Thorac Cardiovasc Surg 1991:3:140-144.

86. McKay RG. PfelTer MA, Pasternak RC: Left ventricular remodeling after myocardial infarct: A corollary to infarct expansion. Circulation 1986;74:693-702.

87. Pfeffer MA, Pfeffer JM: Ventricular enlargement and reduced survival after myocardial infarct. Circulation 1987;75 (suppl IV) 93-97.

88. Pfeffer MA. Braunwald E: Ventricular remodeling after myocardial infarction. Experimental observations and clinical observations. Circulation 1990:81:1 1611172.

89. Benjamin IJ, Schuster EH, Bulkley BH: Cardiac hypertrophy in idiopathic dilated congestive cardiomyopathy: a clinicopathologic study. Circulation 1981: 64:442-447.

90. Pfeffer MA. Lamas GA. Vaughan DE, Parisi AF, Braunwald E: Effect of captopri] on progressive ventricular dilatation after myocardial infarction. $N$ L.ngl J Med 1988:319:80-86.

91. LFP Moreira, Jatene AD: Cardiomyoplasty in dilated cardiomyoplasty. In: Carpentier A. Chachques JC, Grandjean P (Eds.). Cardiomyoplasty. Futura Publishing Company Inc.. Mount Kisco, NY. 1990;171-179.

92. Baker BJ. Wilen MM, Boyd CM, Dinh H, Franciosa JA: Relation of right ventricular ejection fraction to exercise capacity in chronic left ventricular failure. Am J Cardiol 1984:54:596-99. 
93. Franciosa JA, Baker BJ. Seth L: Pulmonary versus systemic hemodynamics in determining exercise capacity of patients with chronic left ventricular failure. Am Heart J 1985; 1 10:807-813.

94. Sullivan MJ, Green HJ, Cobb FR: Skeletal muscle biochemistry and histology in ambulatory patients with long-term heart failure. Circulation 1989;81:518-527.

95. Sullivan MJ, Knight D. Higginbotham MB, Cobb FR: Relation between central and peripheral hemodynamics during exercise in patients with chronic heart failure: Muscle blood flow is reduced with maintenance of arterial perfusion pressure. Circulation 1989;80:769-781.

96. Sullivan MJ, Green HJ, Cobb FR: Altered skeletal muscle metabolic response to exercise in chronic heart failure: relation to skeletal muscle aerobic enzyme activity. Circulation 1991;84:1597-1607.

97. Sullivan MJ, Higginbotham MB, Cobb FR: Exercise training in patients with severe left ventricular dysfunction: hemodynamic and metabolic effects. Circulation 1988;78:506-515.

98. Sullivan MJ. Higginbotham MB. Cobb FR: Exercise training in patients with chronic heart failure delays ventilatory anaerobic threshold and improves submaximal exercise performance. Circulation 1989;79:324-329.

99. Karlsson J: Onset of blood accumulation, exercise capacity, skeletal muscle fibers and metabolism before and after coronary artery bypass grafting. Am $J$ Cardiol 1988;62:108E-1 14E.

100. Stevenson TR, Rohrich RJ, Pollock RA, Dingman RO, Bostwick B: More expertence with the "reverse" latissimus dorsi musculocutaneous flap: Precise location of blood supply. Plast Reconstr Surg 1984:72:237-243.

101. Tobin GR. Schusterman M, Peterson GH, Nichols G. Bland KI: The intramuscular neurovascular anatomy of the latissimus dorsi muscle: The basis for splitting the flap. Plast Reconstr Surg 1981:67:637-641.

102. Adams RJ, Schwartz A: Comparative mechanisms for contraction of cardiac and skeletal muscle. Chest 1980;78 (suppl): 123-139.

103. Parmley WW: Mechanics of ventricular muscle. In: Levine HJ. Gaasch WH (Eds.). The ventricle: Basic and clinical aspects. Martinus Nijhoff Publishers, The Hague. 1985:41-62.

104. Murphy RA: Contraction of muscle cells. In: Berne RM, Levy MN (Eds.). Physiolagy. The CV Mosby Company. St Louise, Washington. DC. Toronto 1988:315342.

105. Buller AJ, Eccles JC, Eccles RM: Interaction between motoneurones and muscles in respect of the characteristic speed of their responses. J Physiol Lond 1960; 150:417-439. 
hapter 2

\section{Changes in Canine Latissimus Dorsi muscle during 24 weeks of continuous electrical stimulation}

Caroline MHB Lucas

Miek G Havenith

Frederik $\mathrm{H}$ van der Veen

Jo Habets

Theo van der Nagel

JM Schrijvers - van Schendel

Olaf CKM Penn

Hein JJ Wellens

Presented in part at the American College of Cardiology. J Am Coll Cardiol 1989,13:214 and at the North American Society of Pacing and Electrophysiology, PACE 1990,13:531.

Published in the J Appl Physiol. 1992;72(3):828-835. 


\title{
Changes in Canine Latissimus Dorsi muscle during 24 weeks of continuous electrical stimulation
}

\begin{abstract}
To study functional, structural and biochemical adaptations to electrical stimulation of striated muscle in a large animal, the canine latissimus dorsi (LD) muscle was conditioned continuously for 24 weeks with an increasing number of pulse bursts (burst duration $=250 \mathrm{msec}$, burst frequency $=30 \mathrm{~Hz}$.

Force measurements in vivo after 12 weeks showed a significant decrease in the ripple (= ratio of interstimulus amplitude to peak force amplitude) from $0.94 \pm 0.03$ to $0.13 \pm 0.08$ (SE, $n=8, \mathrm{P}<0.05$ ) indicating reduction in contractile speed. Also the steep part of the force-frequency relation shifted to lower frequencies.

A significant change in fibre-type composition was seen with both enzyme- and immunohistochemistry, manifested by an increase of type I fibres from $29.5 \pm 2.9 \%$ to $83 \pm 8 \%$ ( $\mathrm{SE}, \mathrm{n}=8, \mathrm{P}<0.05$ ). During this period a transient rise in the number of type IIC/IC fibres (from 3 to $10 \%$ was seen. In the stimulated muscle, capillary-to-fibre ratio increased from $1.9 \pm 0.4$ to $2.7 \pm 0.1$ (SE, $P<0.05$ ). A significant increase in mitochondrial volume percentage was also seen, especially in the peripheral part of the fibre.

Both creatine kinase and lactate dehydrogenase revealed a significant decline in activity within 12 weeks. At the same time a shift in lactate dehydrogenase-isozyme pattern was observed towards the cardiac composition.

No additional changes occurred after 12 weeks of stimulation, indicating that conversion of the canine LD muscle was complete within this period.
\end{abstract}




\section{INTRODUCTION}

After the initial reports of Buller and co-workers in the early 1960s (1) and Salmons and coworkers in 1969 (2), several investigators confirmed profound functional and structural adaptation of skeletal muscle fibres due to electrical stimulation $(3,4,5)$. There have been important clinical uses of electrically stimulated muscle, including correction of spine malalignement in scoliosis patients (6), restoration of walking in paraplegia (7), assistance of cardiac function in dynamic cardiomyoplasty $(8,9,10)$ and a biomechanical component of a counterpulsation device $(11,12,13,14)$. These applications all require the shift from fast-twitch, fatigue-susceptible into slow-twitch, fatigue-resistant muscle fibres.

The purpose of our study was to describe and compare the functional, structural and biochemical changes in latissimus dorsi muscle (LD) of eight dogs during 12 weeks of electrical stimulation. Four of these dogs were followed for an additional 12 weeks. The LD muscle was selected because it is used in studies concerning the effect of dynamic cardiomyoplasty to improve impaired cardiac function.

We believe that this paper adds new information about prolonged stimulation of LD muscle, especially concerning the time course in functional, biochemical and structural changes.

\section{MATERIALS AND METHODS}

Eight mongrel dogs of either sex, with body weights ranging from 25 to 35 $\mathrm{kg}$, were used. All experiments were performed in accordance with the 'Guide for the Care and Use of Laboratory Animals' (15).

\section{Preparation of the dogs}

The animals were anesthetized initially with pentobarbital (Narcovet; 10 $\mathrm{mg} / \mathrm{kg}$ iv), intubated, and then ventilated with oxygen-nitrous oxide and $0.5 \%$ halothane (Fluothane). Subsequently, under sterile conditions and preoperative antibiotic cover (Amfipen, $1000 \mathrm{mg}$ iv), a 10-cm longitudinal skin incision was made in the left midaxillary line. Two intramuscular electrodes (Medtronic SP5528) were sewn $\approx 6 \mathrm{~cm}$ apart into the proximal part of the LD muscle at its thoracic side, perpendicular to the main branches of the left thoracodorsal nerve (16). These electrodes were connected to an implantable Itrel pulse generator (Medtronic SP7420) positioned in a subcutaneous pocket in the interscapular region. All dogs received buprenorfine (Temgesic, $0.6 \mathrm{mg} \mathrm{im}$ ) for 2 days for analgesia. Postoperative antibiotic treatment (Amfipen $1000 \mathrm{mg} \mathrm{im}$ ) was administered on 2 consecutive days.

\section{LD muscle stimulation}

After a recovery period of 2 weeks, conditioning of the LD muscle was started with a progressive stimulation protocol. Because this research was related to a cardiomyoplasty project, we chose a progressive stimulation protocol 
30

comparable to the one used by Chachques et al (17). The following parameters were programmed into the stimulator: an individual pulse width of $210 \mu$ seconds, a burst frequency of $30 \mathrm{~Hz}$ and a burst duration of $250 \mathrm{~ms}$. The pause between the bursts was $1750 \mathrm{~ms}$ at the start of the training period. This pause between bursts was shortened every 2 weeks, resulting in a progressively increasing number of contractions per minute, from 30 to 80 , after 10 weeks (Table 1). The voltage that produced an easily palpable and visible contraction of the entire LD muscle without discomfort to the animal was selected and, if necessary, adapted during the conditioning period. The muscles were stimulated continuously $24 \mathrm{~h} / \mathrm{day}$. In four dogs stimulation was performed for 12 weeks and, in the other four dogs, this period was extended to 24 weeks.

TABLE 1.

Mode of latissimus dorsi muscle stimulation during the periods of conditioning and follow-up.

\begin{tabular}{llll}
\hline week & $\begin{array}{l}T_{\text {on }} \\
\text { (sec) }\end{array}$ & $\begin{array}{l}\mathrm{T}_{\text {oft }} \\
\text { (sec) }\end{array}$ & contractions/min \\
\hline $1-2$ & 0.25 & 1.75 & 30 \\
$3-4$ & 0.25 & 1.25 & 40 \\
$5-6$ & 0.25 & 0.93 & 50 \\
$7-8$ & 0.25 & 0.75 & 60 \\
$9-10$ & 0.25 & 0.62 & 70 \\
$11-24$ & 0.25 & 0.50 & 80 \\
\hline
\end{tabular}

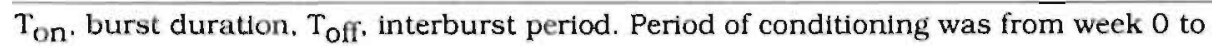
12: follow-up was from week 13 to 24 .

All dogs were investigated at the start and at 4, 8, and 12 weeks; four dogs were also investigated at 24 weeks of stimulation. These investigations included in vivo force measurements of the left LD muscle. Also biopsies from the left and right LD muscle were analyzed to obtain information on fibre type, number of capllaries per muscle fibre, mitochondrial volume density, and enzyme activities.

In vivo force measurements of the LD muscle

After induction of anesthesia with pentobarbital sodium ( $10 \mathrm{mg} / \mathrm{kg}$ iv), the dogs were intubated and ventilated with oxygen-nitrous oxide and $0.5 \%$ Fluothane. Force measurements were performed after positioning of the dog on its right side on the operating table. Subsequently, the left forefoot was connected with straps to a force transducer (Grass FT 10, Grass Instruments, Guincy, MAJ in a position in line with the paw and the LD, that allowed us to measure the force of the LD. The optimal positioning of the paw was tested by administration of twitch contractions at varying length of the LD muscle; the length that yielded maximal force was used for further testing. To restrict compensatory movements of the dog during contraction 
of the left LD muscle, the dog was fixed against two vertical bars, one in front of the thorax and the other at the back of the animal against the scapulae. With this test protocol, the contractions of the muscle can be considered isometric. Subsequently, the following parameters were programmed into the Itrel stimulator: Individual pulse width of $210 \mu$ seconds, a burst duration of $1 \mathrm{~s}$ and an interburst interval of $10 \mathrm{~s}$. A supramaximal voltage (that voltage above which no further rise in isometric force during twitches was measured) and burst frequencies ranging from 2 to $256 \mathrm{~Hz}$ were selected. Force measurements were recorded on a multichannel Schwarzer chart recorder (frequency response $300 \mathrm{~Hz}$ measured for $\pm 3 \mathrm{~dB}$ points) at a paper speed of 25 or $50 \mathrm{~mm} / \mathrm{s}$.

\section{Biopsies}

Immediately after the force measurements, transmural biopsies were obtained from the stimulated and the contralateral LD muscle. These were performed through a small skin incision of $\approx 2 \mathrm{~cm}$ at the midportion of the LD muscle distal to the intramuscular electrodes. Two small samples of $\approx 100 \mathrm{mg}$ each were taken. One sample was quickly frozen in isopentane, quenched in dry ice and stored at $-70^{\circ} \mathrm{C}$ for fibre typing. A very small part of this was used for electronmicroscopic investigation. The second sample was split in two 50-mg portions; one was fixed in neutral buffered $4 \%$ paraformaldehyde and embedded in paraffin. the other was stored at $-70^{\circ} \mathrm{C}$ for measurement of enzyme activities. To minimize damage to the muscle, the biopsies were taken as small as possible, and the localization of subsequent biopsies was varied within the midportion of the muscle, to avoid overlap in biopsy sites.

\section{Histological techniques}

All tissue pieces were embedded in a position suitable for transverse sectioning. Enzyme histochemical fibre typing was performed on serial air-dried transverse cryostat sections of $12-\mu \mathrm{m}$ thickness by use of myosin adenosinetriphosphatase (ATPase) staining, as described by Staron (18) in his modification of Brooke's method (19). A slight $\mathrm{pH}$ adaptation was introduced with preincubation at $\mathrm{pH} 4.15,4.35$ and 10.4 , to improve staining of the type IIC/IC muscle fibres (20). Immunohistochemical fibre typing was performed on 4- $\mu \mathrm{m}$ cryostat sections submerged in cold acetone $\left(-20^{\circ} \mathrm{C}\right)$ for $20 \mathrm{~min}$. After these sections were washed three times for 10 minutes in phosphate-buffered saline (PBS) they were incubated at room temperature with the mouse monoclonal antibody R11D10 (Centocor Europe), diluted 1:2000 in PBS with 1\% bovine serum albumin (BSA) for $60 \mathrm{~min}$. RI ID 10 was raised against human left ventricular heavy-chain myosin (21), an antibody that shows immunoreactivity to type I muscle fibres in human and canine skeletal muscle (20). Sections were again washed three times for 10 min in PBS, followed by application of rabbit anti-mouse antiserum, diluted 1:200 in PBS with 1\% BSA and peroxidase labeled, for $1 \mathrm{~h}$ at room temperature. The peroxidase activity was visualized with diaminobenzidine as chromogen (20). 
In each section, 300 muscle fibres were identified both enzyme histochemically and immunohistochemically. The percentage of types I, II and IIC./IC, as an expression of an intermediate fibre type $(22,23)$ was determined by use of an interactive image analysis system (MOP Videoplan Kontron). Fibre diameter measurements were performed by examining enzyme histochemical (at pH 4.35) and immunohistochemical preparations with a Zeiss microscope connected to a Hitachi FP-10 camera and the MOP Videoplan Kontron analysis system. At a final magnification of $\times 250$, the smallest diameter of each fibre was measured (24).

To obtain information about the number of capillaries per muscle fibre, 300 muscle fibres with adjacent capillaries were counted at the start, at 12 weeks ( $n=8$ ) and 24 weeks ( $n=4)$ of stimulation. This was done both on enzyme histochemical preparations at $\mathrm{pH} 4.15$ and on immunohistochemical preparations with the use of monoclonal antibodies to the basement membrane component type IV collagen, which enables the visualization of basement membranes of both muscle fibres and capillaries (25).

Electronmicroscopic investigation was performed in biopsies obtained from the stimulated LD muscle at 0 and 12 weeks in eight dogs and at 24 weeks in four dogs. These biopsies were fixed in a solution of $2.5 \%$ glutaraldehyde

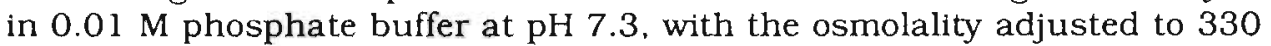
mosmole by addition of sucrose. Transverse sections were routinely stained with lead citrate and uranyl acetate and examined with a Philips 300 electronmicroscope. From each muscle biopsy six fields at the peripheral and six at the central part were selected and photographed at a final magnification of $\times 14,000$. A digitizer (MOP Videoplan Kontron) with a cross-hair cursor was used. The perimeter of mitochondria and myofibrillar bundles present in a reference space, including those crossing the top-left boundaries, was outlined manually. Reproducibility was found to be within $\pm 2 \%$ for ten repeated measurements. Volume fraction of mitochondria and myofibrils was calculated for peripheral and central fields in the muscle fibres.

\section{Enzyme activity}

The LD muscle samples obtained in the biopsy sessions, as described above, were allowed to thaw at room temperature and subsequently homogenized in $20 \mathrm{ml}$ of Tris(hydroxymethyl)aminomethane-(Tris) $\mathrm{HCl}$ buffer $(0.05 \mathrm{M}, \mathrm{pH}$ 8.5), in bursts of 7-s with intervals of 7-s with a Janke Kunkel Ultraturrax (type 18-10). The buffer contained $0.05 \mathrm{M} \mathrm{NaCl}, 0.015 \mathrm{mM}$ pyridoxal-5phosphate and $3 \mathrm{mM}$ mercaptoethanol. After homogenization, samples were sonicated for 3 minutes, in 5-s bursts at 5-s intervals with a MSE Mark II 150-W disintegrator. During homogenization and sonication the tubes were kept on ice. From the thoroughly mixed suspension a 2 -ml sample was taken for the determination of dry weight and total protein content. The activities of creatine kinase (CK) and lactate dehydrogenase (LDH) were determined spectrophotometrically with commercially available test kits (NAC-activated CK-test. Merck Diagnostica; optimized LDH test. Boehringer) on a centrifugal analyzer (Cobas Bio System, Hoffman La Roche). 
LDH-isozymes were measured (26) after electrophoresis (Beckmann Instruments). Activities were expressed in micromoles of substrate converted per minute per litre (U/liter). Total protein content was determined with the Folin reagent. Dry weight was determined by lyophilization of $1 \mathrm{ml}$ of suspension in a Leyboldy-Heraeus vacuum freeze drier (GT 2) after previous freezing of samples at $-70^{\circ} \mathrm{C}$.

\section{Statistical analysis}

A repeated-measures analysis of variance test was performed on our whole data set, followed by a paired Student's $t$ test, to locate significant differences between the control measurements and measurements done during the training protocol. Differences were accepted to be significant at $\mathrm{P}<0.05$. Values are presented as mean $\pm \mathrm{SE}$.

\section{RESULTS}

All dogs tolerated the period of electrical stimulation very well, ambulation not being disturbed by a continuously contracting left LD muscle. Small increments in voltage were necessary to maintain a visible and palpable contraction of the entire muscle, especially during the lst weeks of stimulation. These increments resulted in a voltage of $3.2 \pm 0.5 \mathrm{~V}$ after 24 weeks of conditioning, in contrast to the $2.9 \pm 0.3 \mathrm{~V}$ at the beginning of muscie conditioning.

\section{Force measurements}

The left LD muscle showed an increase in contraction and relaxation time. reflected in the ripple, which is defined as the ratio of the interstimulus amplitude to the peak force amplitude (figure 1). The most impressive change in the ripple occurred within the first 4 weeks of electrical stimulation; after this period the decline was less pronounced. No significant change in ripple was observed after 12 weeks of muscle conditioning.

The force-frequency relation showed no significant change in frequencies at which maximal force of the LD muscle was obtained (figure 2). However, from 10 to $25 \mathrm{~Hz}$, a significant increase in relative maximal force occurred in the conditioned LD muscle. Maximal tetanic force $(11.7 \pm 1.1 \mathrm{~kg}$. at 0 weeks and $9.9 \pm 1.7 \mathrm{~kg}$. ( $(\mathrm{n}=8)$ after 12 weeks) did not change significantly. After 24 weeks the measured maximal force in the four remaining dogs was $9.4 \pm 1.1 \mathrm{~kg}$.

\section{Fibre typing}

As shown before, type I fibres reacted intensely on enzyme histochemistry at pH 4.1 and 4.35 and showed intense immunoreactivity with R1 1D 10 (27). The type IC and IIC fibres were characterized by moderate ATPase activity at $\mathrm{pH} 4.35$ and slight (IC) to intense (IIC) activity at $\mathrm{pH}$ 10.4. The immunoreactivity of the type IC/IIC fibres with RIIDIO was less pronounced than that of type I fibres because of the reduced amount of type I myosin 
34:

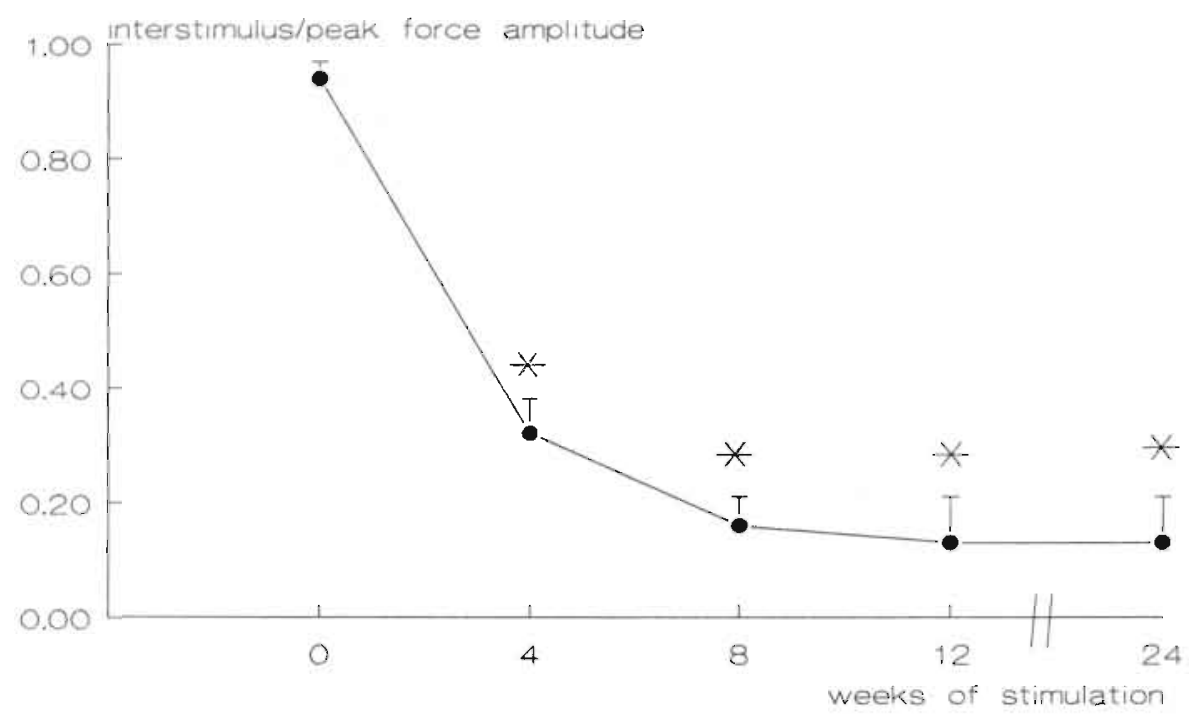

FIGURE 1 .

Ripple (ratio of interstimulus to peak force amplitude) of LD muscle calculated from $10 \mathrm{~Hz}$ bursts. Values are means $\pm S E$. *: Significant change of ripple compared with preconditioning value, $P$ $<0.05$

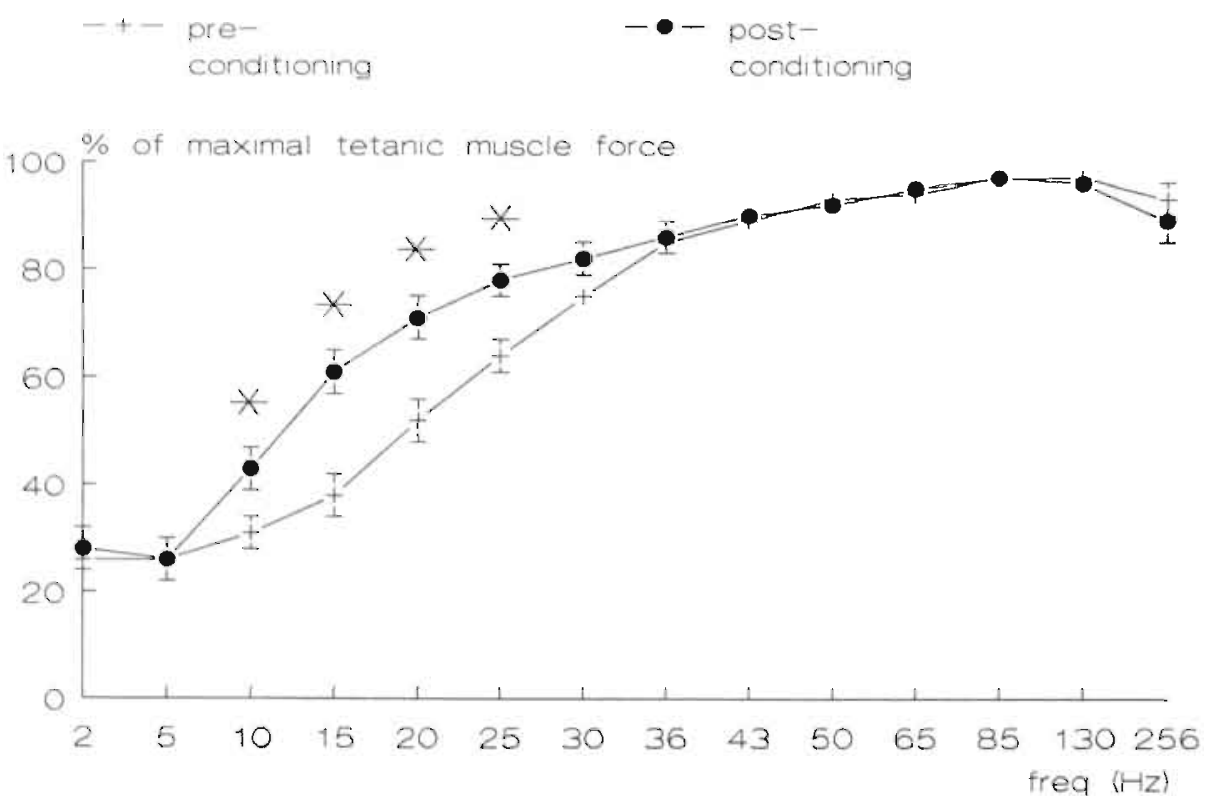

FIGURE 2.

Muscle force (means \pm SE) normalized to maximum tetanic tension, measured during test. stimulation with bursts at frequencies from 2 to $256 \mathrm{~Hz}$ both before and after a conditioning period of 12 weeks. ": Significantly different from value before stimulation, $P<0.05$. 

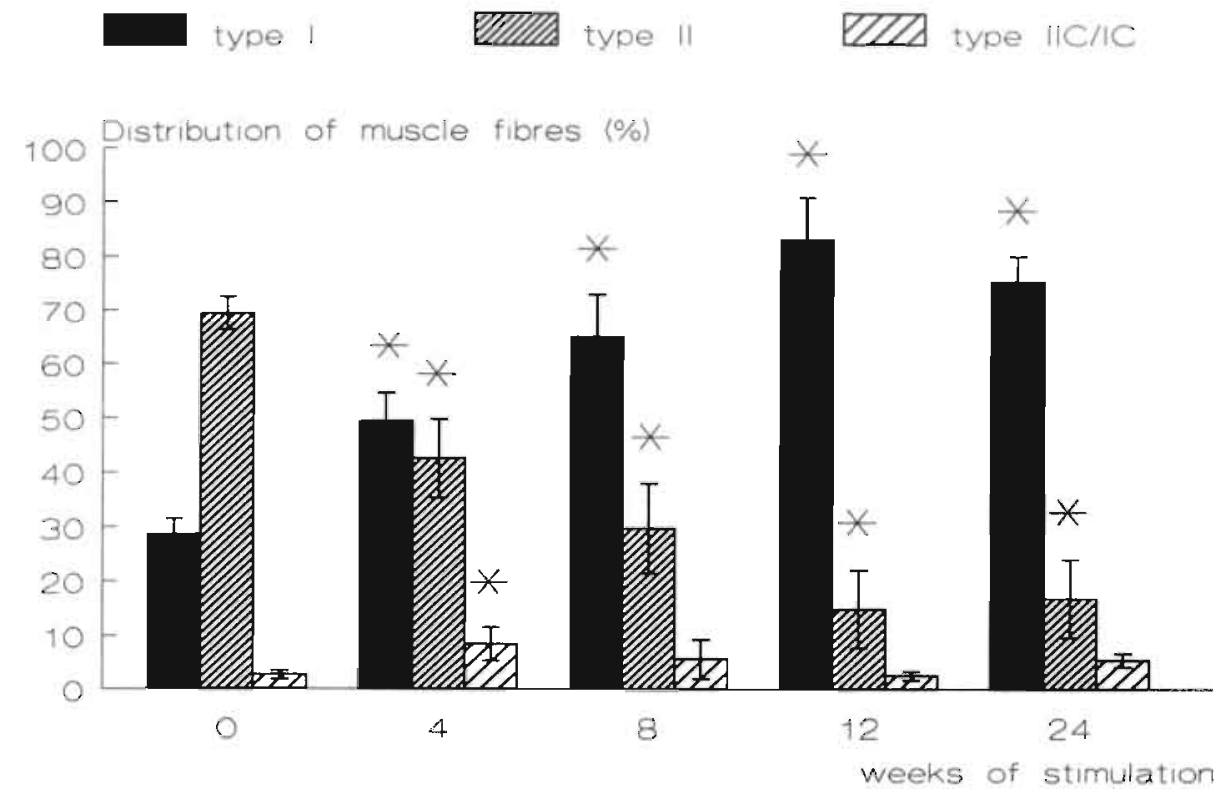

FIGURE 3.

Distribution of fibre types in LD muscle during 24 weeks of electrical stimulation. *: Significantly different from preconditioning value, $P<0.05$.

heavy chain (MHCI) in these fibres (18). Enzyme histochemically-identified type ILA fibres, further referred to as type II. lacked any reactivity with R11D10. Classical type IIB fibres cannot be found in dogs, as demonstratecl in earlier studies $(28,29)$. Enzyme histochemistry and immunohistochemistry yielded identical results. Before the start of LD muscle stimulation, a distribution of $28.5 \pm 2.9 \%$ type I, $69.2 \pm 3 \%$ type II and $2.6 \pm 0.8 \%$ type IIC/IC was observed in both LD muscles. Muscle conditioning resulted in a progressive increase of type I fibres to $82.8 \pm 7.0 \%$ and a decrease in type II fibres to $14.8 \pm 7.0 \%$ after 12 weeks (figure 3 ). Concomitantly, an increase in type IIC/IC fibres was observed during the first 8 weeks. An example of muscle fibre changes as obtained with immunohistochemical and enzyme histochemical methods in a LD muscle stimulated for 24 weeks is shown in figure 4 . In contrast to other investigators $(30,31)$, we did not observe a change in fibre type in the contralateral nonstimulated muscle during the conditioning period. No significant effect of continuous electrical stimulation on muscle fibre diameter was observed after either 12 weeks or 24 weeks (figure 5).

Capillary-to-fibre ratio increased significantly within 12 weeks in eight dogs. from $1.9 \pm 0.4$ to $2.7 \pm 0.1$. At 24 weeks this ratio was $2.8 \pm 0.1$ in the four remaining dogs, which was not significantly different from the value found at 12 weeks.

Total mitochondrial volume per muscle fibre increased significantly during the first 12 weeks of stimulation, both in the central and in the peripheral 

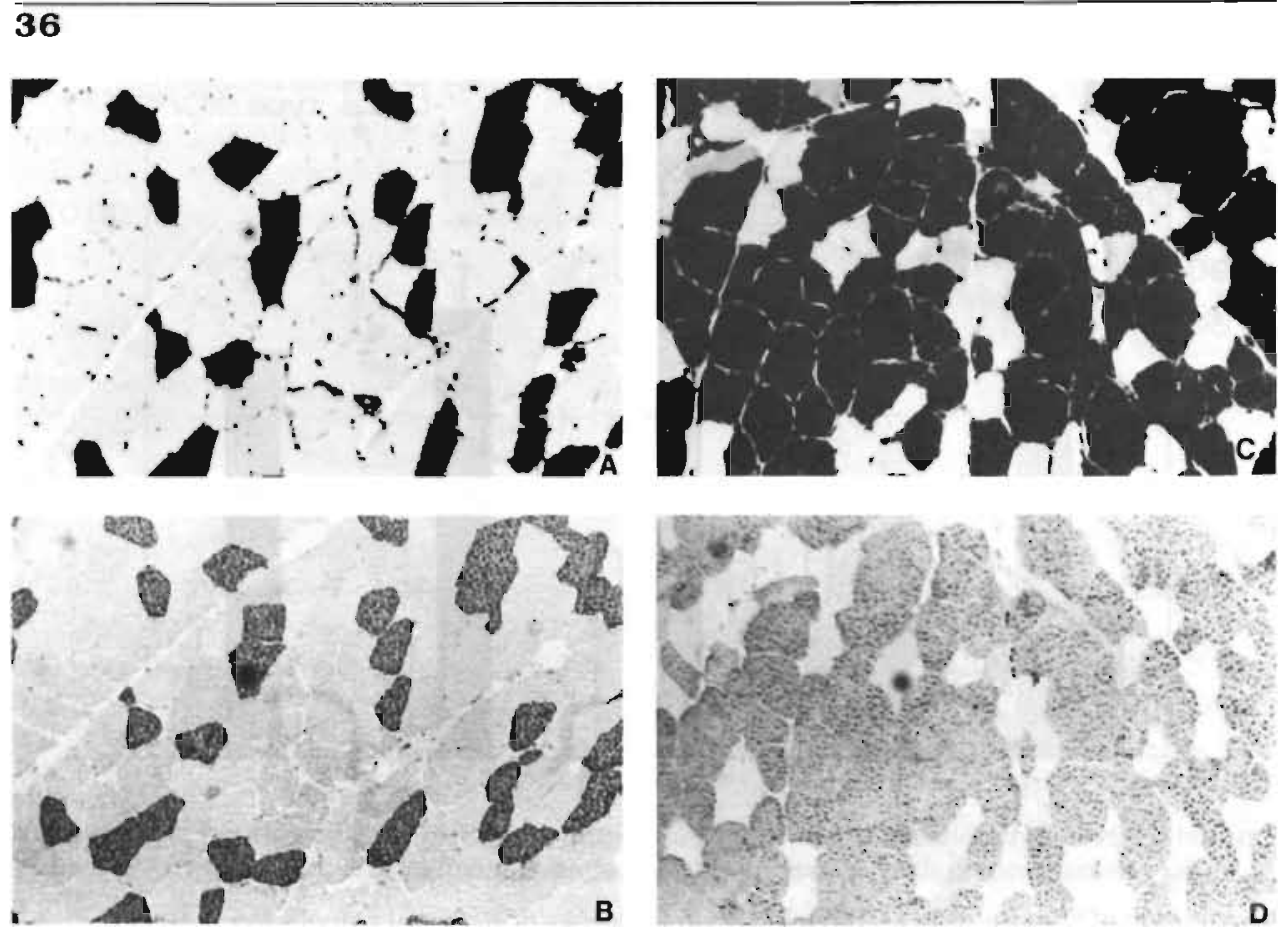

FIGURE 4.

Serial sections of unconditioned ( $A$ and $B$ ) and conditioned $(C$ and $D) L D$ muscle after a stimulation period of 24 weeks. Sections $A$ and $C$ are stained with myosin adenosinetriphosphatase after preincubation at $p H 4$ 4.4. Sections $B$ and $D$ are stained with immunoperoxidase with R1 $1 D 10$ monoclonal antibody raised against myosin heavy chain type I fibres (magnification $\times 210$ ). Note that 2 staining methods show identical fibres.

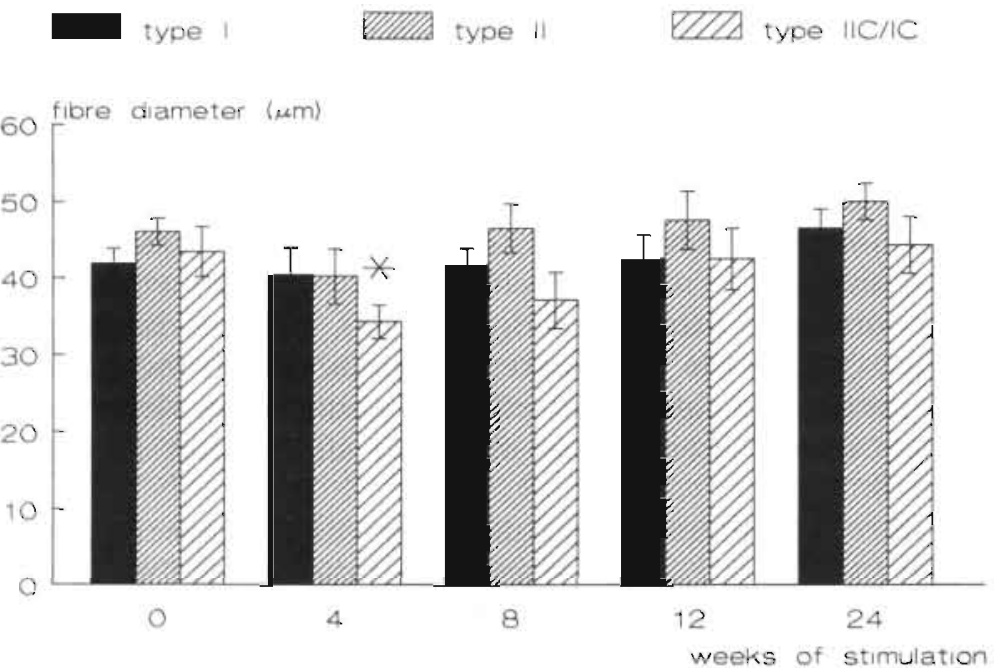

FIGURE 5.

Fibre diameter (means \pm SE) of type I, type II and intermediate type fibres during 24 weeks of stimulation. * Significantly different from value before stimulation, $P<0.05$. 
TABLE 2.

Percentage of mitochondrial volume and of myofibrillar volume in the nonstimulated and the stimulated latissimus dorsi muscle.

\begin{tabular}{lcllll}
\hline & & \multicolumn{2}{l}{ Myofibrillar (\%) } & \multicolumn{2}{l}{ Mitochondrial (\%) } \\
\cline { 3 - 6 } & $\mathrm{n}$ & Central & Peripheraj & Central & Peripheral \\
\hline $\begin{array}{l}\text { Nonstimulated LDi } \\
\text { Stimulated LD }\end{array}$ & 8 & $72.9 \pm 2.9$ & $72.1 \pm 2.0$ & $3.4 \pm 0.53$ & $5.5 \pm 0.5$ \\
12 weeks & 8 & $70.0 \pm 1.2$ & $64.2 \pm 1.0^{*}$ & $5.3 \pm 0.6^{*}$ & $10.3 \pm 1.1^{*}$ \\
24 weeks & 4 & $66.8 \pm 1.3$ & $65.3 \pm 1.6^{*}$ & $6.6 \pm 0.8^{*}$ & $9.3 \pm 2.3^{*}$
\end{tabular}

Values are means $\pm \mathrm{SE}$. LD; latissimus dorsi. Central and peripheral areas of muscle. fibres were used for morphometric analyses. * Significant difference from control.

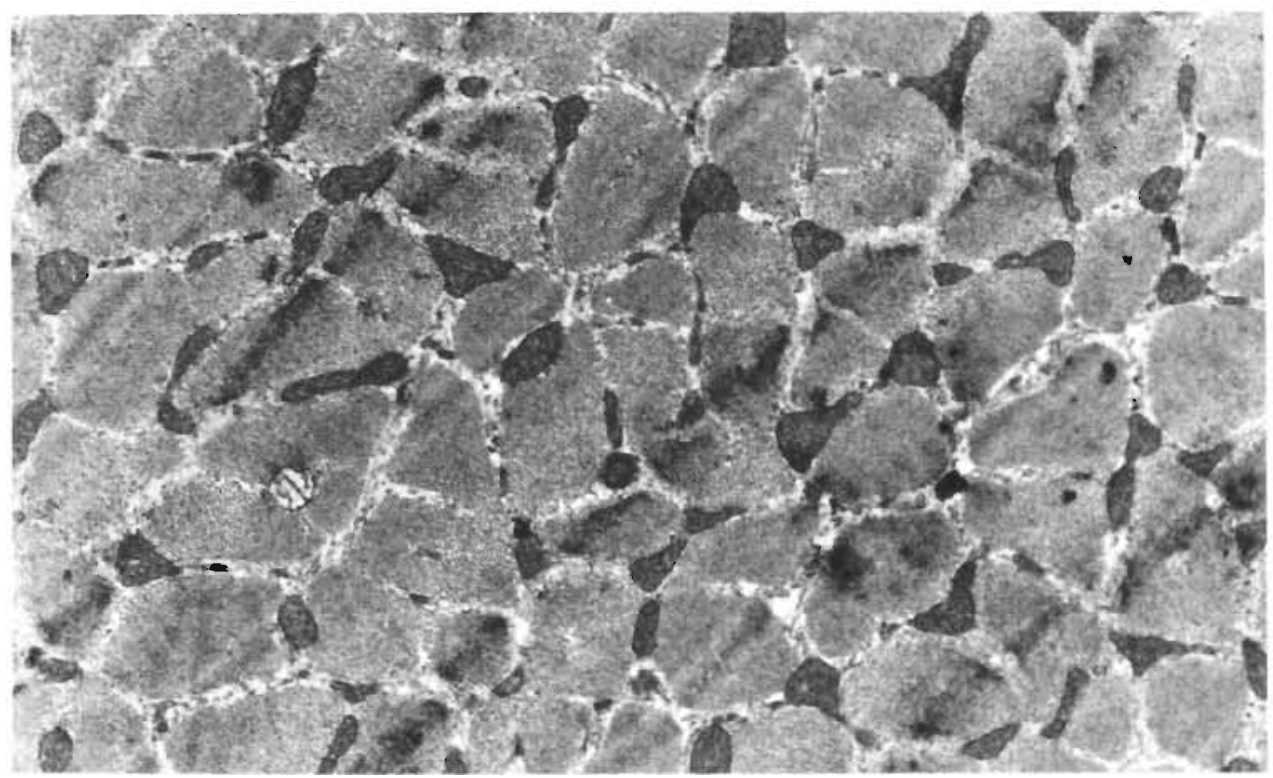

FIGURE 6.

Transverse section of $L D$ muscle fibre after 24 weeks of electrical stimulation showing abundance of mitochondria (magnification $\times 14.000$ ).

part of the fibres (Table 2). No additional increase in mitochondrial volume occurred after 12 weeks of muscle stimulation. Figure 6 demonstrates the abundance of mitochondria after conditioning. Clustering of mitochondria was observed in peripheral parts of some muscle fibres which is in accord with the report by Eisenberg and Salmons (32).

LDH and CK activity in the stimulated LD muscle gradually decreased compared with the contralateral muscle (figure 7). Either enzyme demonstrated a tendency to a decrease within 4 weeks of stimulation; however, for 
38

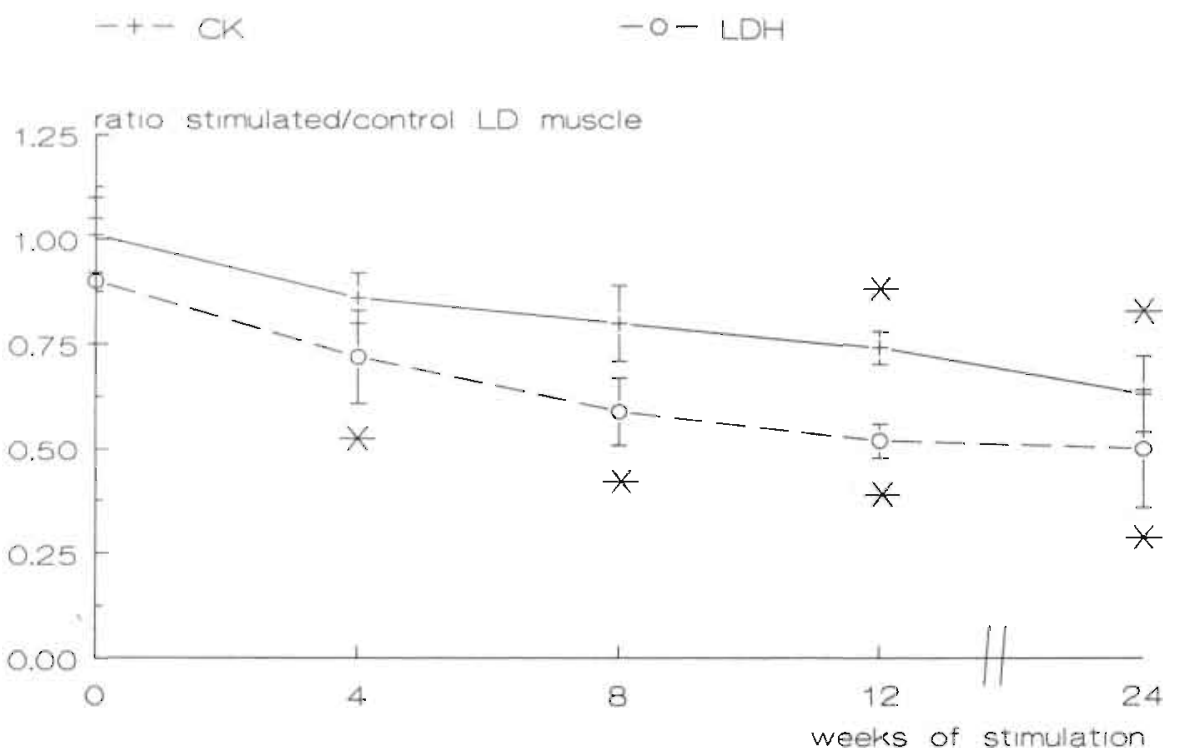

FIGURE 7.

Tissue enzyme activities of lactate dehydrogenase (LDH) and creatine kinase (CK) expressed as ratio of stimulated muscle to control $L D$ muscle. "Significantly different from initial activity before the start of stimulation, $P<0.05$.

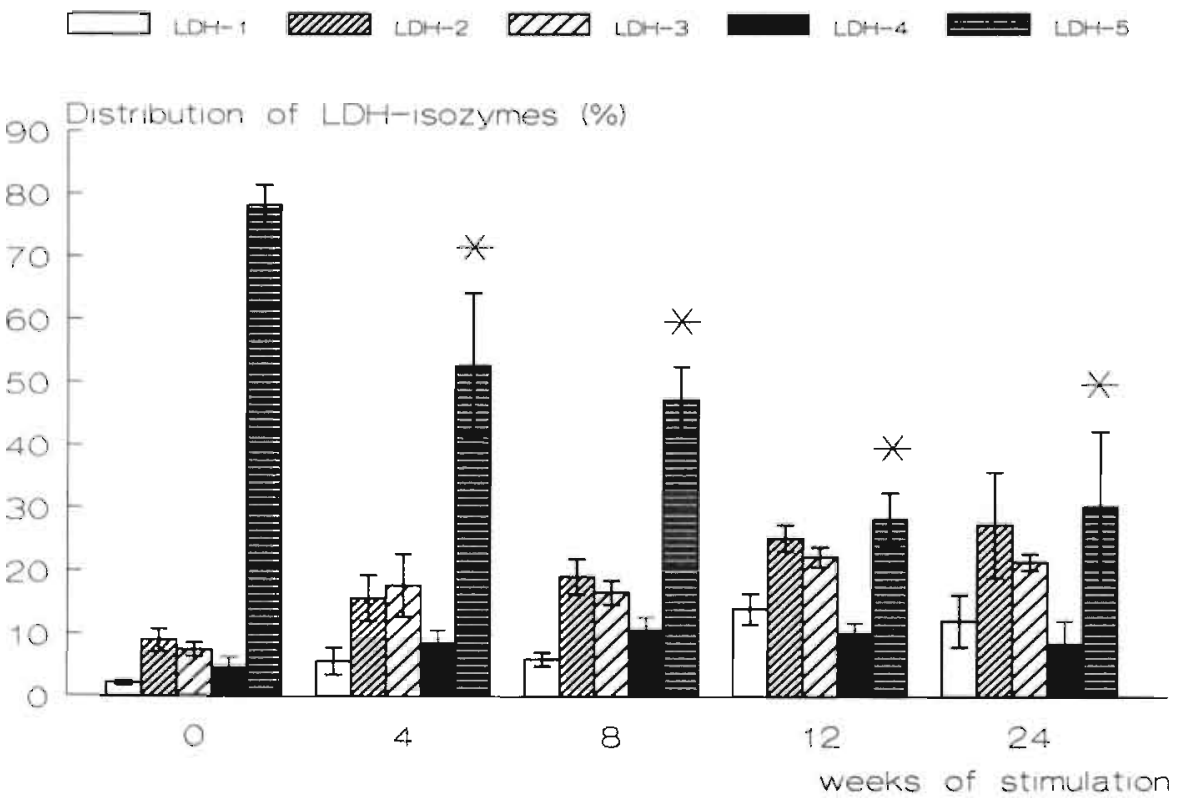

FIGURE 8 .

LDH-isozyme distribution as percentage of total LDH-activity during 24 weeks of muscle stimulation. "Signfficant decrease in LDH type 5 from value obtained at start of stimulation. $P$ $<0.05$. 
CK this was not significant before 12 weeks of LD muscle conditioning. There was no additional change in activity of either enzyme between 12 and 24 weeks of stimulation. A clear change in the distribution of LDH-isozymes was observed, from a predominance of LDH type 5 to a significant increase of LDH type 1, 2 and 3 within 4 weeks of stimulation (figure 8 ).

\section{DISCUSSION}

Chronic electrical stimulation of canine skeletal muscle resulted in significant changes in functional, biochemical and structural characteristics within 12 weeks. These findings are in accordance with experiments performed in small animals, such as rats and rabbits $(3,5,8,32-35)$.

Our experimental setup for in vivo force measurements on the LD muscle appeared adequate for obtaining information about the change in contraction and relaxation speed, as demonstrated in the ripple and force during progressive conditioning of the LD muscle. Chronic stimulation resulted in a decrease in the ripple of the stimulated canine LD muscle within a few weeks. This rapid decrease in contraction speed and relaxation speed is probably due to changes in $\mathrm{Ca}^{2+}$ handling $(4.5)$, although we did not measure it.

Changes in the myosin composition, as reflected in the appearance of slow myosin isoforms, occur at a slower rate during the transformation process of the muscle $(3,35)$. Our results of fibre typing indicate that this phenomenon can also be observed in the canine LD muscle. The appearance of IIC/IC fibres, in particular at $4-8$ weeks of stimulation, strongly suggests progressive transformation of muscle fibres. These fibres were also desicribed by Staron and co-workers (36), who explained their appearance as a temporary phenomenon during the transformation of type IIA, via the fibre types IIC and IC, to type I. Pette and co-workers (4) observed $\approx 10 \%$ type: IIC/IC fibres during electrical stimulation in rabbits, which is comparable to our findings. No additional increase in type I fibres, could be demonstrated between 12 and 24 weeks of stimulation.

In contrast to findings reported by Acker et al. (11) we did not observe complete conversion to type I fibres. In their experiments, however, the LD muscle was used to construct a skeletal muscle ventricle, with the LD muscle completely detached from its insertion. Because of this detachment, they could stimulate the LD muscle at a supra-maximal voltage without disturbing the gait pattern of the animal, which was not the case in our model. Also, stimulation via intramuscular electrodes, rather than a cuff electrode around the nerve, might be less efficient to stimulate all nerve branches (37). Both possible explanations are supported by our observation that after 24 weeks of stimulation some muscle fascicles were exclusively composed of type I fibres adjacent to non-transformed fascicles.

In the present study, conditioning of the LD muscle did not result in a significant change in frequency at which the relative maximal force was obtained $(85 \mathrm{~Hz})$. However, conditioning-induced leftward displacement of 


\section{$\mathbf{4 0}$}

the steep part of the force-frequency relation curve has an important implication for medical practice in which skeletal muscle is electrically stimulated. In the frequency range of $10-25 \mathrm{~Hz}$, programming of a lower burst frequency after conditioning of the muscle results in a higher relative force, which has a beneficial effect on the longevity of the pacemaker and constitutes a less strenuous burden for the muscle.

The absence of changes in fibre diameter of type I fibres due to electrical stimulation of canine LD muscle, confirms observations by Clark and co-workers (38). Their results and ours, however, seem to be in contrast to other reports that describe an overall decrease in diameter of all fibre types in the muscle and a substantial loss of force after a period of continuous electrical stimulation $(31,39,40,41,5)$. This apparent discrepancy could be due to differences in stimulation: single pulses vs bursts of pulses. It was shown that the application of bursts of stimuli, with a short interburst interval, rather than application of single-pulse stimulation, resulted in less pronounced or absent changes in diameter, loss of muscle mass, and loss in tetanic force $(16,30,31,42,43)$. In addition, both the duration of the burst and the number of bursts per minute appear to be important, because a significant decrease in maximal force of conditioned canine LD muscle was observed when a relatively short burst duration of $100-125 \mathrm{~ms}(13,44)$ was used concomitant with a high number of bursts.

The capillary-to-fibre ratio in our dogs before and after muscle stimulation was higher than that for extensor digitorum longus and flexor hallucis muscles in rabbits (45). Moreover, the stimulation-induced increase in this ratio is less prominent in canine LD muscle than it is in rabbit skeletal muscle. This is most likely related to the high initial oxidative capacity of canine skeletal muscle, which lacks genuine type IIB fibres. Cooper and Hudlicka (46) suggested that the effect of a small increase in capillary-tofibre ratio is an improved dispersion of blood flow between the fibres, which facilitates the transport of oxygen and metabolic substrates and improves rephosphorylation of ADP and creatine. This is in accordance with the findings of Clark and co-workers (38) who used ${ }^{31} \mathrm{P}$ nuclear magnetic resonance to demonstrate an increased capacity of oxidative phosphorylation in conditioned canine LD muscle. They suggested that this was partly due to an increase in mitochondrial volume. We indeed found a significant rise in the subsarcolemmal mitochondrial volume (Fig. 6, and Table 2). The presence of a large subsarcolemmal mitochondrial volume in these continuously stimulated muscles could contribute to facilitation of transsarcolemmal transport of metabolites from the circulation to the contracting muscle cells.

The decrease of muscle LDH activity and the shift in the isozyme pattern of LDH are comparable to observations during the transformation of the glycolytic type IIB fibres to the oxidative type I fibres in rabbits $(33,47,48)$. Even in type ILA muscle fibres, LDH type 5 is the predominant LDH isozyme type (49), which explains our finding that in canine LD muscle $[\approx 70 \%$ type 
ILA fibres) electrical stimulation results in a shift to LDH-isozyme type 1, 2 and 3. Thus the LDH-isozyme pattern seen after continuous electrical stimulation is more similar to the one found in cardiac tissue.

This study also shows the occurrence of a decline in CK-enzyme activity that is comparable to observations of rabbit tibialis anterior muscle $(33,35)$. Although no effort was made to separate cytosolic and mitochondrial CK-activity in our dog experiments, rabbits show a relative increase in mitochondrial CK-activity (50). This enzyme fraction is important in the creatine phosphate shuttle (51) and therefore might contribute to the improved metabolic capacity of conditioned skeletal muscle.

The variables selected for the evaluation of skeletal muscle adaptation all showed maximal transformation within 12 weeks of stimulation, a time period that is consistent with the results of other studies of canine L.D muscle $(12,13,37,38,44,52)$.

We conclude that continuous electrical stimulation of canine LD muscle results within 12 weeks in a transformation to a muscle consisting of mainly type I fibres, with an increased capillary-to-fibre ratio, an elevated mitochondrial volume density, and a decrease in CK- and LDH-activity concomitant with a shift in $\mathrm{LDH}$-isozyme distribution. 


\section{REFERENCES}

1. Buller AJ, Eccles JC, Eccles RM: Interaction between motoneurones and muscles in respect of the characteristic speed of their responses. J Physiol Lond 1960; 150:417-439.

2. Salmons S, Vrbova G: The influence of activity on some contractile characteristics of mammalian fast and slow muscles. J Physiol Lond 1969;210:535549 .

3. Pette D: Activity-induced fast to slow transition in mammalian muscle. Med Sci Sports Exc 1984:16:517-528.

4. Pette D. Vrbova G: Invited review: Neural control of phenotypic expression in mammalian muscle fibres. Muscle \& Nerve 1985:8:676-689.

5. Salmons S, Henriksson J: The adaptive response of skeletal muscle to increased use. Muscle \& Nerve 1981;4:94-105.

6. Axelgaard J. Brown JC: Lateral electrical surface stimulation for the treatment of progressive idiopathic scoliosis. Spine 1983;8:242-260.

7. Marsolais EB, Kobetic R: Functional electrical stimulation for walking in paraplegia. J Bone It Surg Am 1987;69:728-733.

8. Carpentier A. Chachques JC: The use of stimulated skeletal muscle to replace diseased human heart muscle. In: Chiu RC-J (Ed.). Biomechanical cardiac assist: Cardiomyoplasty and muscle-powered devices. Futura Publishing Company, Mount Kisco, NY, 1986;85-102.

9. Magovern G. Heckler F, Park SB, Christlieb IY, Burkholder J, Maher T, Benckart D. Magovern Jr GJ. Kao R: Paced skeletal muscle for dynamic cardiomyoplasty. Ann Thorac Surg 1988;45:614-619.

10. Molteni L, Almada $\mathrm{H}$ : Clinical cardiac assist with synchronously stimulated skeletal muscle. J Thorac Cardiovasc Surg 1988:95:940-941.

11. Acker MA. Hammond RL, Mannion JD. Salmons S, Stephenson LW: An autologous biologic pump motor. J Thorac Cardiovasc Surg 1986:92:733-746.

12. Anderson WA. Bridges CR, Chin AJ, Andersen JS. Acker MA. Hammond RL, Dimeo F, Calahan PT, Gale DR, Brown WE. Stephenson LW: Long-term neurostimulation of skeletal muscle: Its potential for a tether-free biologic cardiac assist device. PACE 1988:11:2128-2134.

13. Chiu RC-J, Walsh GL, Dewar ML, de Simon J-H, Khalafalla AS, Ianuzzo D: Implantable extra-aortic balloon assist powered by transformed fatigue-resistant skeletal muscle. J Thorac Cardiovasc Surg 1987:94:694-701.

14. Kochamba G. Desrosiers C, Dewar M. Chiu RC-J: The muscle-powered dualchamber counterpulsator: Rheologically superior implantable cardiac assist device. Ann Thorac Surg 1988:45:620-625.

15. National Academy of Sciences: Guide for the Care and Use of Laboratory Animals. DHHS publication No. NIH 85-23, revised 1985.

16. Chachques JC, Grandjean P. Schwartz K, Mihaileanu S, Fardeau M, Swynghedauw B. Fontaliran F, Romero N, Wisnewsky C, Perrier P. Chauvaud S, Bourgeois I. Carpentier A: Effect of Latissimus Dorsi dynamic cardiomyoplasty on ventricular function. Circulation 1988:78(suppl III):203-216.

17. Chachques JC, Grandjean PA. Carpentier A: Dynamic Cardiomyoplasty: Experimental Cardiac Wall Replacement with a stimulated skeletal muscle. In: Chiu RC-J (Ed.). Biomechanical cardiac assist: Cardiomyoplasty and musclepowered devices. Futura Publishing Company. Mount Kisco, NY. 1986:59-84.

18. Staron RS. Pette D: Correlation between nyofibrillar ATPase activity and myosin heavy chain composition in rabbit muscle tibres. Histochemistry 1986:86: 19-23. 
19. Brooke MH, Kaiser KK: Muscle fibre types: how many and what kind. Arch Neurol 1970:23;369-379.

20. Havenith MG, Visser R, Schrijvers - van Schendel JM, Bosman FT: Muscle fibre typing in routinely processed skeletal muscle with monoclonal antibodies. Histochemistry 1990;93:497-499.

21. Khaw BA, Mattis JA. Melincoff G, Straus HW, Gold HK, Haber E: Monoclonal antibody to cardiac myosin: imaging of experimental myocardial infarction. Hybridoma 1984:3:1 1-23.

22. Billeter R, Heizmann CW, Howald H, Jenny E: Analysis of myosin light and heavy chain types in single human skeletal muscle fibres. Eur $\mathrm{J}$ Biochem 1981: 116:389-395.

23. Staron RS. Pette D: Nonuniform myosin expression along single fibres of chronically stimulated and contralateral rabbit tibialis anterior muscles. Pflügers Arch 1987:409:67-73.

24. Dubowitz V: Muscle Biopsy. A practical approach. Second Edition. Balliere Tindall. London. 1982;89.

25. Havenith MG, Cleutjens JPM, Beek C, Van der Linden E. De Goei AFPM, Bosman FT: Human specific anti-type IV collagen monoclonal antibodies, characterization and immunohistochemical application. Histochemistry 1987:87:123-128.

26. Van der Veen FH, Visser R, Willems GM, Kop-Klaassen B, Hermens WT: Myocardial enzyme depletion in infarcted human hearts: infarct size and equivalent tissue mass. Cardiovasc Res 1988;22:611-619.

27. Havenith MG, Van der Veen FH, Glatz JFC, Lucas C, Schrijvers-van Schendel JMC. Penn OCKM. Wellens HJJ: Monitoring of muscle fibre type of canine latissimus dorsi muscle during chronic electrical stimulation by enzyme- and immunohistochemistry. In: Chiu RC-J, Bourgeois IM (Eds.). Transtormed muscle for cardiac assist and repair. Futura Publishing Company, Mount Kisco, NY, 1990;53-64.

28. Maxwell LC, Barclay JK, Mohrman DE, Faulkner JA: Physiological characteristics of skeletal muscles of dogs and cats. Am J Physiol 1977;233:C14-C18.

29. Snow DH, Billeter R, Mascarello F. Carpene E, Rowlerson A, Jenny E: No classical type IIB fibres in dog skeletal muscle. Histochemistry 1982;75:53-65.

30. Donselaar Y, Eerbeek D, Kernell D, Verhey BA: Fibre sizes and histochemical staining characteristics in normal and chronically stimulated fast muscle of cat. J Physiol Lond 1987:382:237-254.

31. Eerbeek O, Kernell D, Verhey B: Effects of fast and slow patterns of tonic long-term stimulation on contractile properties of fast muscle in the cat. J Physiol Lond 1984:352:73-90.

32. Eisenberg BR, Salmons S: The reorganization of subcellular structure in muscle undergoing fast-to-slow type transformation. Cell Tiss Res 1984:220:22 1-230.

33. Henriksson J, Chi M-Y, Hintz CS, Young DA, Kaiser KK, Salmons S, Lowry OH: Chronic stimulation of mammalian muscle: Changes in enzymes of six metabolic pathways. Am J Physiol 1986;251:C614-C632.

34. Klug G. Wiehrer W, Reichmann H, Leberer E, Pette D: Relationships between early alterations in parvalbumins, sarcoplasmatic reticulum and metabolic enzymes in chronically stimulated fast twitch muscle. Pflügers Arch 1983: 399:280-284.

35. Pette D, Müller W, Leisner E, Vrbova G: Time dependent effects on contractile properties, fibre population, myosin light chains and enzymes of energy metabolism in intermittently and continuously stimulated fast twitch muscles of the rabbit. Pllügers Arch 1976;364:103-112. 
36. Staron RS, Golsch B. Pette D: Myosin polymorphism in single fibres of chronically stimulated rabbit fast-twitch muscle. Pflügers Arch 1987:408:444-450.

37. Badylak SF, Hinds M. Geddes LA: Comparison of three methods of electrical stimulation for converting skeletal muscle to a fatigue resistant power source suitable for cardiac assistance. Ann Biomed Eng 1990;18:239-250.

38. Clark BJ, Acker MA. McCully K, Subramanian HV, Hammond RL, Salmons S, Chance B. Stephenson LW: In vivo ${ }^{31}$ P-NMR spectroscopy of chronically stimulated canine skeletal muscle. Am J Physiol 1988:254:C258-C.266.

39. Mannion JD, Bitto T, Hammond RL, Rubinstein NA, Stephenson LW: Histochemical and fatigue characteristics of conditioned canine latissimus dorsi muscle. Circ Res 1987:58:298-304.

40. Mannion JD, Velchik M, Hammond R. Alavi A. Mackler T. Duckett S, Staum M. Hurwitz S, Brown W, Stephenson LW: Effects of collateral blood vessel ligation and electrical conditioning on blood flow in dog Latissimus Dorsi muscle. J Surg Res 1989;47:332-340.

41. Walsh GL, Dewar ML, Khalafalla AS. Neilson IR. De Simon J-H, Chiu RC-J: Characteristics of transformed fatigue-resistant skeletal muscle for long-term cardiac assistance by extra-aortic balloon counterpulsation. Surg Forum 1986; 37:205-207.

42. Ferguson AS, Stone HE, Roessmann U, Burke M. Tisdale E, Mortimer JT: Muscle plasticity: Comparison of a $30-\mathrm{Hz}$ burst with $10-\mathrm{Hz}$ continuous stimulation. $\mathrm{J}$ Appl Physiol 1989:66:1143-1151.

43. Hudlicka O, Tyler KR. Srihari T, Heilig A, Pette D: The effect of different patterns of long-term stimulation on contractile properties and myosin light chains in rabbit fast muscles. Pflügers Arch 1982;393:164-170.

44. Leirner AA, Moreira LFP, Chagas ACP, Cestari IA, Oshiro MS, Nakayama E. da Luz PL. Stolf AG, Jatene AD: Biomechanical circulatory assistance: Importance of aerobic capacity of normal and conditioned skeletal muscle. Trans Am Soc Artil Int. Organs 1988;34:716-720.

45. Brown MD, Cotter MA. Hudlicka O. Vrbova G: The effects of different patterns of muscle activity on capillary density. mechanical properties and structure of slow and fast rabbit muscles. Pflügers arch 1976:361:241-250.

46. Cooper J. Hudlicka O: Effect of the change in the capillary bed induced by long-term stimulation on muscle fatigue. J Physiol Lond 1976:263:155-156P.

47. Pette D. Smith ME. Staudte HW, Vrbova G: Effects of long-term electrical stimulation on some contractile and metabolic characteristics of fast rabbit muscles. Pflügers Arch 1973;338:257-272.

48. Reichmann H, Hoppeler H, Mathieu-Costello O, von Bergen F. Pette D: Biochemi$\mathrm{cal}$ and ultrastructural changes of skeletal muscle mitochondria after chronic electrical stimulation in rabbits. Pflügers Arch 1985:404:1-9.

49. Leberer E. Pette D: Lactate dehydrogenase isozymes in type I, ILA and IIB fibres of rabbit skeletal muscles. Histochemistry 1984:80:295-298.

50. Schmitt T. Pette D: Increased mitochondrial creatine kinase in chronically stimulated fast-twitch rabbit muscle. FEBS 1985:188:341-344.

51. Jacobus WE: Respiratory control and the integration of heart high-energy phosphate metabolism by mitochondrial creatine kinase. Ann Rev Physiol 1985:47:707-725.

52. Bridges CR, Brown WE, Hammond RL, Anderson DR, Anderson WA, Dimeo F, Stephenson LW: Skeletal muscle ventricles: improved performance at physiologic preloads. Surgery 1989:106:275-282. 
hapter 3

\section{Adaptations of Goat Latissimus Dorsi muscle using two different protocols of continuous electrical stimulation}

Caroline MHB Lucas

Marie-Louise Dubelaar

Frederik $\mathrm{H}$ van der Veen

Elisa N Kloosterman - Castro-Ravelo

Miek G Havenith

Jo Habets

Theo van der Nagel

Olaf CKM Penn

Hein JJ Wellens

Presented at the North American Society of Pacing and Electrophysiology. PACE 1991;14:691.

Submitted for publication. 


\title{
Adaptations of Goat latissimus dorsi muscle using two different protocols of continuous electrical stimulation
}

\begin{abstract}
Electrical stimulation of the latissimus dorsi (LD) muscle in situ was performed in 6 goats over a 12 week period, as a model for LD muscle conditioning after cardiomyoplasty.

Two different stimulation protocols were used. One protocol (A) is currently in use in patients after a cardiomyoplasty procedure, starting with 1 pulse stimulation. The second protocol (B) started with a burst of 3 pulses. The number of pacing stimuli was progressively increased during 12 weeks, ending with burst stimulation ( 80 bursts/minute; 6 pulses per burst, interpulse intervals of $33 \mathrm{msec}=$ burst frequency 30 $\mathrm{Hz}$ ) in both protocols.

Force measurements in vivo showed both a significant decrease in the ripple (= ratio of interstimulus amplitude to peakforce amplitude, measured at a burst frequency of $10 \mathrm{~Hz}$ ) from $0.88 \pm 0.16$ to $0.01 \pm 0.01$ (P< 0.05 ) and a leftward shift of the force-frequency relation after 12 weeks. Fatigue-resistance improved significantly, as demonstrated by the percentage of the remaining force after an 8 minutes fatigue-test, which increased from $30 \pm 10 \%$ to $79 \pm 4 \%(\mathrm{P}<0.05)$. At 4 weeks of stimulation, the changes in ripple and fatigue-resistance tended to be more pronounced using protocol B.

Histological evaluation revealed typical signs of muscle transformation: Increased mitochondrial volume, nearly all type I fibres, increased capillary-to-fibre ratio and an increase in endomyseal connective tissue area/muscle fibre area. Also a significant decrease in muscle fibre area appeared obvious.
\end{abstract}


Enzyme-activity measurements in LD muscle biopsies revealed a significant decline in activity of both creatine kinase and lactate dehydrogenase within 12 weeks. At the same time a shift in lactate dehydrogenase isozyme pattern was observed, resembling that of cardiac muscle.

We conclude that in situ stimulation of latissimus dorsi muscle in goats results in mechanical, histological and biochemical changes, which are comparable to those described in small animals. Both protocols used, induce these changes within 12 weeks, with protocol $\mathrm{B}$ showing a tendency of a faster transformation.

\section{INTRODUCTION}

During recent years an increasing number of publications have appeared. describing a new surgical technique, called 'dynamic cardiomyoplasty' (1-5). During this procedure the left latissimus dorsi (LD) muscle is wrapped around a failing heart and stimulated synchronously with ventricular contraction. Following a period of electrical stimulation using an increasing number of stimuli, the wrapped muscle is conditioned to contract with a maximum of 100 times per minute after 12 weeks (3). Unfortunately, it is difficult to evaluate the mechanical, histochemical and ultrastructural changes that occur during and after this conditioning period, when the skeletal muscle is wrapped around the heart. Therefore, we studied these adaptations in the left LD muscle by stimulating the muscle in situ during a period of 12 weeks. The effect of stimulation on histochemical and ultrastructural changes was studied by comparing the stimulated with the non-stimulated contralateral LD muscle. We also evaluated the effect of two different stimulation protocols on the mechanical properties of the muscle in this model, during and at the end of the conditioning period.

\section{MATERIALS AND METHODS}

Six female goats with body weights ranging from 29 to $54 \mathrm{~kg}$ were used. All experiments were performed in accordance with the Guide for the Care and Use of Laboratory Animals' (6).

\section{Preparation of the goats}

The animals were anesthetized with thiopental (Nesdonal $15 \mathrm{mg} / \mathrm{kg}$ body weight) into the jugular vein, intubated and ventilated with oxygen-nitrous oxide (1:2) and 1.5\% halothane (Fluothane). A $15 \mathrm{~cm}$ longitudinal skin incision was made in the left midaxillary line under sterile conditions. Two intramuscular electrodes (Medtronic SP5528) were inserted $=6 \mathrm{~cm}$ apart into the proximal part of the LD muscle on the thoracic side. perpendicular to the main branches of the left thoracodorsal nerve (3). An implantable Itrel 
pulse generator (Medtronic SP7420), positioned in a subcutaneous pocket in the interscapular region, was connected to these electrodes. One dose of Amfipen (1000 $\mathrm{mg} \mathrm{im}$ ) was administered pre-operatively and one postoperatively for antibiotic cover, and analgesia was provided with buprenorfine (Temgesic $0.6 \mathrm{mg} \mathrm{im}$ ) for two days.

\section{LD muscle stimulation}

As shown in Table 1, conditioning of the LD muscle was started two weeks after the operation. Two different protocols were used. Three goats were used for each of the two protocols. Protocol A is routinely used in patients after a dynamic cardiomyoplasty procedure, starting with 1 pulse stimulation (3). In protocol B, conditioning of the LD muscle was started with a short burst of 3 pulses. Both protocols finally ended with a burst of 6 pulses with interpulse intervals of $33 \mathrm{~ms}$ (= burst frequency $30 \mathrm{~Hz}$ ) and a maximum rate of 80 bursts per minute. The voltage, that produced an easily palpable and visible contraction of the LD muscle without discomfort to the animal, was selected and, when necessary, voltage level was adapted during the conditioning period. The muscles were stimulated continuously 24 hours a day for a period of 12 weeks.

\section{In vivo force measurements of the LD muscle}

Contractile force of the left LD muscle was evaluated at $0,4,8$ and 12 weeks of electrical stimulation.

After induction of anesthesia with thiopental (Nesdonal; $15 \mathrm{mg} / \mathrm{kg}$ iv), the goats were intubated and ventilated with oxygen-nitrous oxide and $1.5 \%$ Fluothane. They were rigidly fixed with straps on their right side to avoid movement during contraction of the left LD muscle. Concomitantly, the left forefoot was connected to a force displacement transducer (Grass FT 10 , Grass Instruments, Quincy, MA] with the left paw and left LD muscle in a position in line with the transducer, to measure the force of the stimulated skeletal muscle. First, isometric twitch contractions at varying lengths of the LD muscle were obtained. The length which yielded maximal force was used for further testing. Subsequently the pulse stimulator was programmed as follows: 1. Individual pulse width $210 \mu$ second. 2 . Burst duration 1 $\mathrm{sec}$. 3. Interburst interval $10 \mathrm{sec}$. The same voltage, as programmed for conditioning of the LD muscle, was used for these measurements. Burst frequencies from 2 to $256 \mathrm{~Hz}$ were selected. All force measurement sessions were ended with a fatigue test of 8 minutes duration. For this test, a burst was used consisting of 6 pulses with interpulse intervals of $33 \mathrm{~ms}$ (= burst frequency $30 \mathrm{~Hz}$ ). The $L D$ muscle was stimulated at a rate of 100 bursts/ minute. Voltage and pulse width were not changed. Force, positive $\mathrm{dF} / \mathrm{dt}$ and negative $\mathrm{dF} / \mathrm{dt}$ of the $\mathrm{LD}$ muscle were recorded on a multichannel Schwarzer (frequency response $300 \mathrm{~Hz}$ measured for $\pm 3 \mathrm{~dB}$ points) at a paper speed of $25 \mathrm{~mm} / \mathrm{sec}$. 
TABLE 1.

Shape of the two latissimus dorsi stimulation protocols $A(n=3)$ and $B(n=3)$.

\begin{tabular}{lllll}
\hline & week & pulses/burst & $\begin{array}{c}\text { interpulse interval(msec]/ } \\
\text { burst frequency }(\mathrm{Hz})\end{array}$ & bursis/min \\
\hline A & $1-2$ & 1 & & 50 \\
& $3-4$ & 2 & $100 / 10$ & 50 \\
& $5-6$ & 3 & $67 / 15$ & 50 \\
& $7-8$ & 6 & $33 / 30$ & 50 \\
& $9-10$ & 6 & $33 / 30$ & 60 \\
& $11-12$ & 6 & $33 / 30$ & 80 \\
\hline B $1-2$ & 3 & $33 / 30$ & 30 \\
& $3-4$ & 3 & $33 / 30$ & 40 \\
& $5-6$ & 3 & $33 / 30$ & 50 \\
$7-8$ & 6 & $33 / 30$ & 50 \\
$9-10$ & 6 & $33 / 30$ & 80 \\
$11-12$ & 6 & $33 / 30$ & 80 \\
\hline
\end{tabular}

\section{Biopsies}

Before taking biopsies from left and right LD muscle, both muscles were exposed completely to have an optimal view of the LD muscle. Transmural biopsies were obtained from the midportion of the stimulated and from the same area of the non-stimulated contralateral LD muscle, distal to the intramuscular electrodes. All tissue pieces were carefully positioned for transverse sectioning.

For muscle fibre typing and the determination of the number of capillaries per muscle fibre, one biopsy was quickly frozen in isopentane, quenched in dry ice and stored at $-70^{\circ} \mathrm{C}$.

To evaluate the amount of connective tissue in the LD muscles, a second biopsy was taken from the adjacent location, fixed in $4 \%$ paraformaldehyde and subsequently embedded in paraffin.

For electronmicroscopic investigation of mitochondrial and myofibrillar volume, biopsies $(2 \times 2 \times 2 \mathrm{~mm})$ were obtained from the left and right LD muscle and fixed in a solution of $2.5 \%$ glutaraldehyde in $0.01 \mathrm{M}$ phosphate buffer at $\mathrm{pH} 7.3$ with the osmolality adjusted to 330 mosmole by addition of sucrose.

Specimen for the determination of lactate dehydrogenase (LDH) and creatine kinase (CK) enzyme-activity were taken from left and right LD muscles and immediately stored at $-70^{\circ} \mathrm{C}$ pending subsequent analyses.

Histochemical techniques

Combined immunohistochemical fibre typing (7) and enzymehistochemical capillary staining using alkaline phosphatase (8) was performed on $8 \mu \mathrm{m}$ cryostat sections submerged in cold acetone $\left(-4^{\circ}\right.$ C) for 5 minutes. Subse- 


\section{0}

quently, the sections were incubated for 60 minutes in a medium containing nitroblue tetrazolium, 5-bromo-4 chloro-3-indolyl phosphate. After washing these sections 3 times during 5 minutes each in tris(hydroxymethyl) aminomethane buffered saline (TBS), they were incubated at room temperature with the mouse monoclonal antibody R11D10 (Centocor Europe), 1:10000 diluted in TBS with $1 \%$ bovine serum albumin (BSA) for 45 minutes. This antibody is raised against human left ventricular heavy chain myosin and has immunoreactivity to type I muscle fibres in human, canine and goat skeletal muscle (9). Sections were washed again 3 times during 5 minutes in TBS, followed by application of 1:200 diluted (in TBS with 1\% BSA) peroxidase labeled rabbit anti-mouse antiserum for 1 hour at room temperature. The peroxidase activity was visualized by diaminobenzidine as chromogen. Finally. all sections were stained for 5 minutes using diluted eosine.

In each section, 300 muscle fibres were identified immunohistochemically. The percentage of types I. II and IIC/IC as an expression of an intermediate fibre type $(10,11)$ was counted using an interactive image analysis system (MOP Videoplan Kontron). The cross-sectional area of 300 individual muscle fibres in randomly distributed fascicles was measured and expressed as the diameter of the circle of an equivalent area (Dcircle). This was performed by examining immunohistochemical preparations with a Zeiss microscope in connection with a Hitachi FP- 10 camera and the MOP Videoplan Kontron analysis system. Concomitantly, the number of capillaries per muscle fibre was checked in the same specimen. All capillaries were counted which were adjacent to the muscle fibres used for fibre typing and fibre area measurements.

The Picrosirius-Polarization method, staining collagen type I, II and III, was used to demonstrate the amount of endomyseal connective tissue within the left and right LD muscle (12). Three micrometer sections were incubated for 5 minutes in aqueous phosphomolybdic acid (13), followed by staining in $0.1 \%$ Sirius red F3BA (C.I. 35780. Polysciences, Norhampton, UK), in saturated aqueous picric acid (90 minutes). Subsequently, the sections were washed with $0.01 \mathrm{~N} \mathrm{HCl}$, cleared and mounted. Imaging of the amount of Sirius red staining skeletal muscle tissue was performed using an image analyzer (CAS 200, Inc, Becton and Dickinson) with its Quantitative Nuclear Antigen program. Two masks are used in this program. One mask is used to define a threshold for the total area of skeletal muscle tissue. The other masks defines the threshold for the measurement of the amount of Sirius red staining area, which is expressed as the percentage of the total measured area. Five skeletal muscle fascicles were chosen randomly from each specimen. The amount of endomyseal connective tissue was measured at 5 different sites within each selected fascicle.

\section{Electronmicroscopic investigation}

Transverse sections of both left and right LD muscle were routinely stained with lead citrate and uranyl acetate and examined using a Philips 300 electronmicroscope. Six fields were selected at the peripheral and 6 at the 
central part from each muscle slide and photographed at a magnification of $\mathrm{x} 14.000$. Measurements of the perimeter of the mitochondria and myofibrillar bundles were done as described before, using a digitizer (MOP Videoplan Kontron) with a cross-hair cursor (14).

Enzyme activity measurements

All LD muscle samples were handled exactly as has been described before (14).

\section{Statistical analysis}

When appropriate repeated-measures analysis of variance was used followed by Student's t, test to analyze all data. Differences were accepted to be significant at $\mathrm{P}<0.05$. Values are presented as mean $\pm \mathrm{SD}$.

\section{RESULTS}

None of the goats showed signs of discomfort due to the continuously contracting left latissimus dorsi muscle. The voltage needed to obtain a visible and palpable contraction of the muscle was $2.6 \pm 0.4 \mathrm{~V}$ during the entire conditioning period of 12 weeks. There was no need for increments nor decrements in stimulation voltage.

In vivo force measurements of the LD muscle

A progressive increase in contraction and relaxation time occurred during the conditioning period of 12 weeks. As shown in figure 1 , this is reflected in the ripple, defined as the ratio of the interstimulus amplitude to the peak force amplitude using a burst frequency of $10 \mathrm{~Hz}(2)$. At 4 weeks of training, this ratio was significantly lower in the goats which were trained according to protocol B. Concomitantly, a significant difference was seen in fatigueresistance between both groups. This is demonstrated in figure 2 , showing the remaining force at 8 minutes presented as a percentage of the force at the start of the test. No differences in both parameters between both groups were seen at 8 and 12 weeks of conditioning.

Electrical stimulation resulted in a leftward shift in force-frequency relation (figure 3). As there was no significant difference between both groups after 12 weeks of conditioning, data of all 6 goats are summarized in this figure. Concomitantly a decrease in maximal force from $7.1 \pm 5.0$ to $3.6 \pm 1.1 \mathrm{~kg}$ (NS, $\mathrm{n}=6$ ) was observed. This was accompanied by a significant improvement in fatigue-resistance as demonstrated in figure $4 \mathrm{a}$. In this figure, the complete fatigue-test of 8 minutes both before and after 12 weeks of LD muscle stimulation is shown, summarizing the data of all 6 goats before and 5 goats after conditioning. As shown, percentage remaining positive $\mathrm{dF} / \mathrm{dt}$ (figure $4 \mathrm{~b}$ ) and negative $\mathrm{dF} / \mathrm{dt}$ (figure $4 \mathrm{c}$ ) also improved significantly. However the training period of the LD muscle did not prevent an important decrease in these last two parameters during the test. In one goat (subjected to protocol A), which did not show striking abnormalities in mechanical 


\section{2}
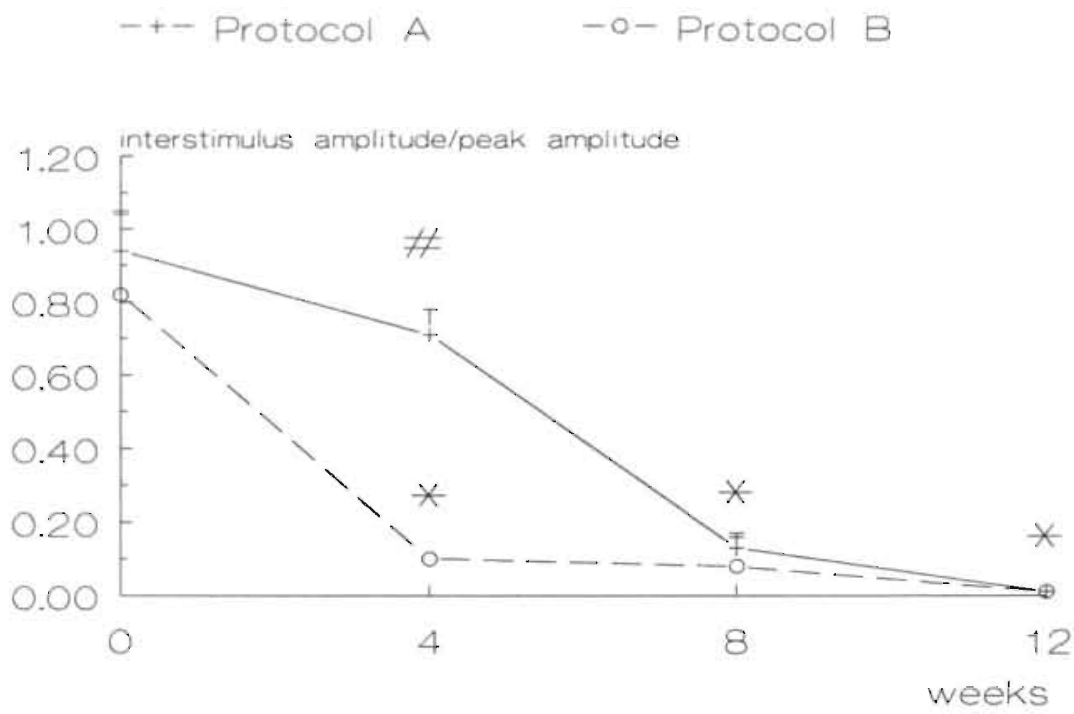

FIGURE 1 .

Ripple (ratio of interstimulus to peak force amplitude) of $L D$ muscle, calculated from $10 \mathrm{~Hz}$ bursts for both protocols $A$ and $B$. as observed during the 12 weeks stimulation period. Values are means $\pm S D$. "Significant change of the ripple compared with preconditioning value, $P<0.05$. \# Significant difference between protocols $A$ and $B . P<0.05$.
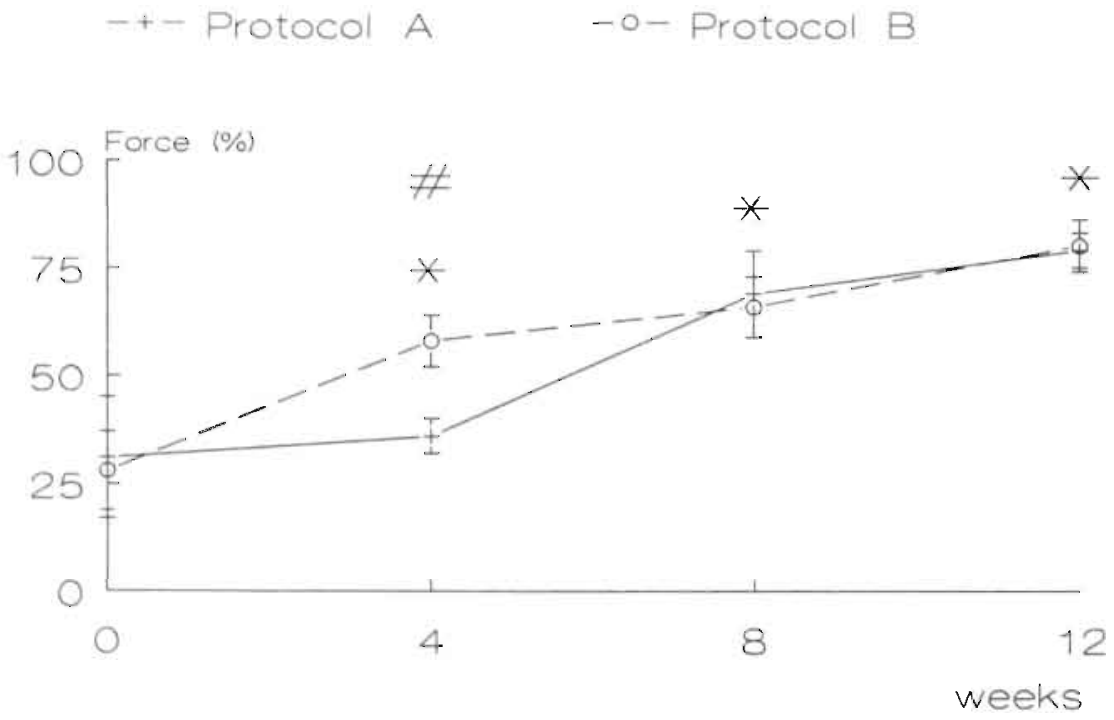

FIGURE 2.

Remaining force at the end of an 8 minutes fatigue test. pictured as a percentage of the force at the beginning of the test at 0.4 .8 and 12 weeks of stimulation. Significant change when compared with the preconditioning value, $P<0.05$. \#ignificant difference between protocols $A$ and $B, P<0.05$. 


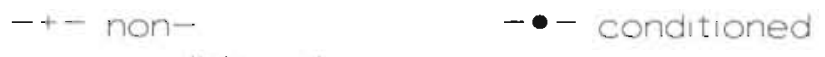

conditioned

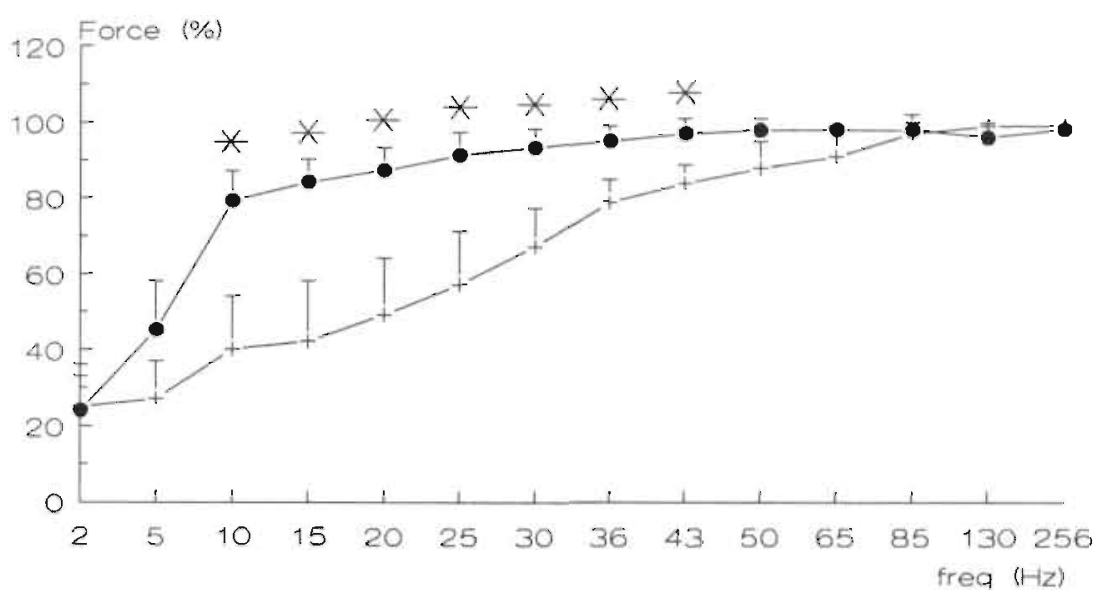

FIGURE 3.

Muscle force (means $\pm S D$ ) normalized to maximum tetanic tension, measured during test stimulation with bursts at frequencies from 2 to $256 \mathrm{~Hz}$, both before and after a conditioning period of 12 weeks. $n=6$ : ${ }^{*}$ Significant difference between non-conditioned and conditioned $L D$ muscles, $P<0.05$.

properties at 8 weeks of conditioning, the relaxation of the stimulated muscle was severely impaired already after 1 minute during the fatigue-test at 12 weeks. Therefore, results at 12 weeks in this goat are omitted from the figure. This LD muscle demonstrated severe pathological changes as will be described in the next section.

\section{Histochemical techniques}

In 5 goats no detrimental effect on the histological architecture of the muscle was seen, except for some slight increase in fatty cells in the interfascicular connective tissue. All muscle cells had a rounded appearence in comparison to the more angulated non-stimulated muscle fibres. One goat, however, which was trained according to protocol $\mathrm{A}$, demonstrated a large number of atrophic cells, intermingled with hypertrophic cells. Also other signs of muscular cell destruction were seen like central nuclei and coiled fibres (15). For this reason, results from this goat are omitted from the histological data. RIIDI0, staining myosin heavy chain from type I fibres, demonstrated almost complete conversion to type I fibres ( $98 \pm 3 \%$ ) compared to the control LD muscle $(19 \pm 4 \%)$. This full transformation was also seen in the goat with the pathological changes in the stimulated LD muscle. The amount of intermediate staining fibres (IC/IIC) appeared negligible.

Capillary-to-fibre ratio increased significantly in both groups resulting in a value of $1.80 \pm 0.35(n=5)$ compared to $1.02 \pm 0.12(n=6, P=0.01)$ in the non-stimulated muscle. 


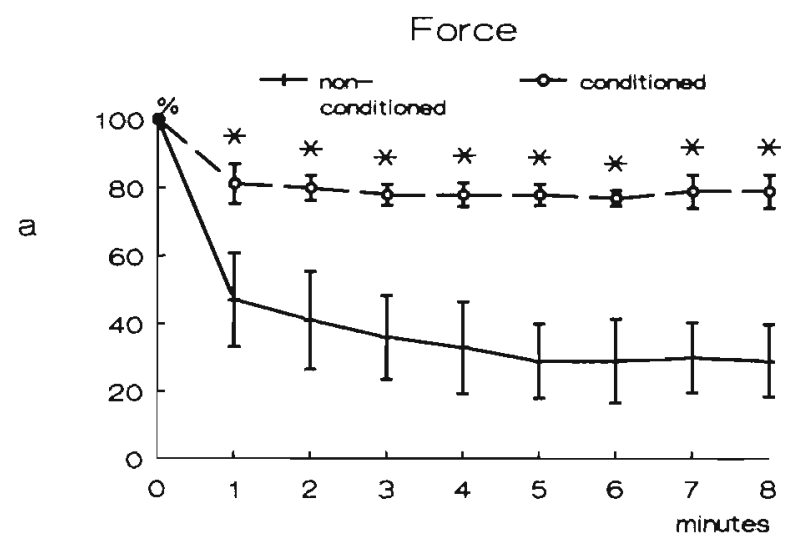

$\mathrm{dF} / \mathrm{dt}+$

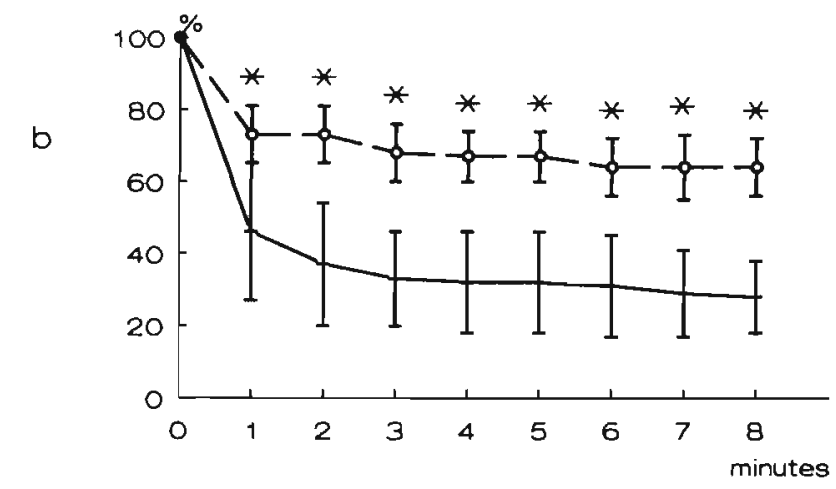

$d F / d t-$

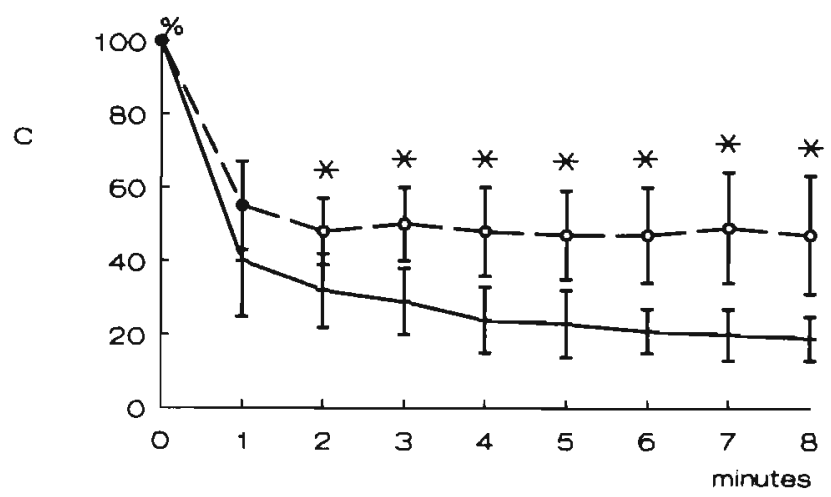

FIGURE 4.

Force (a), positive $\mathrm{dF} / \mathrm{dt}(\mathrm{b})$ and negative $\mathrm{dF} / \mathrm{dt}$ (c) measured during a fatigue test of 8 minutes, both before and after a conditioning period of 12 weeks. Non-conditioned: $n=6$. conditioned: $n$ =5: "Significant difference between non-conditioned and conditioned LD muscles, $P<0.05$. 


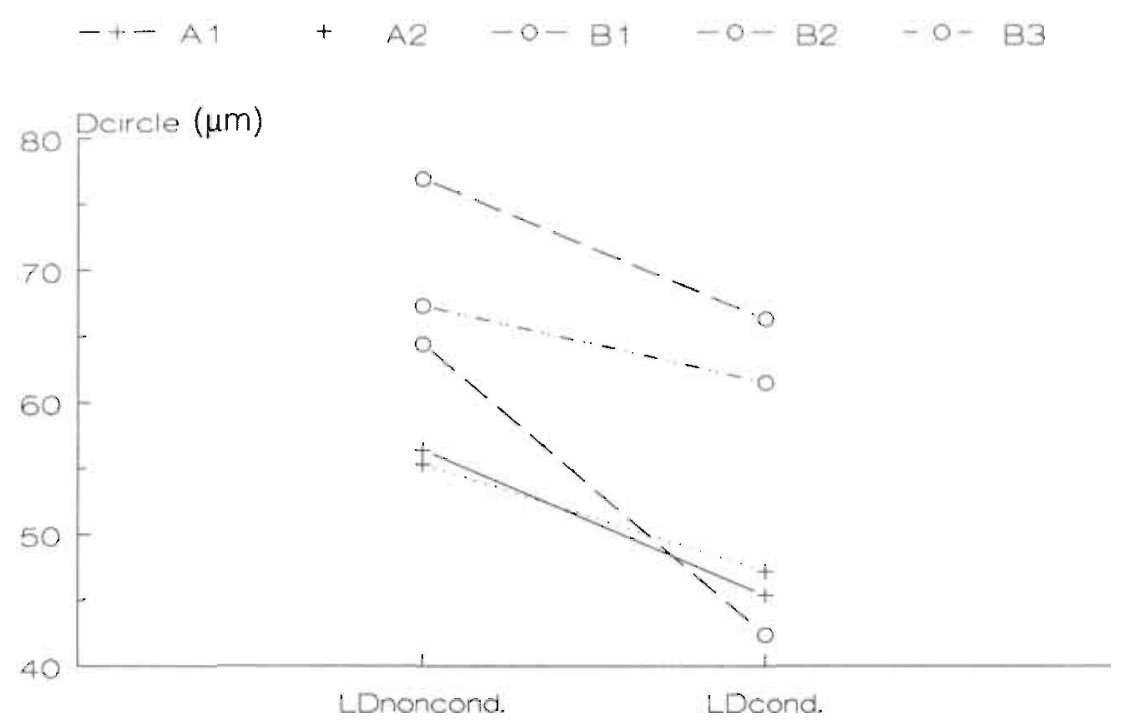

FIGURE 5.

Dcircle from type I muscle fibres from non-conditioned and conditioned LD muscles from 5 individual goats. A 1 and $A 2$ are data from 2 goats belonging to protocol $A$, whert 'us B1, B2 and $B 3$ are data from those 3 goats stimulated according to protor'il $B$.

A clear decrease was seen in the diameter of an equivalent circle, irrespective of the protocol of stimulation. In figure 5 Dcircle of the type I fibres of left and right LD muscle are compared for all individual goats, demonstrating for all animals $(\mathrm{n}=5)$ significant differences with a P-value $<0.001$. Summarizing all data. Dcircle of the type I fibres decreased from 64.1 \pm 7.9 $(\mathrm{n}=6)$ to $52.6 \pm 9.5 \mu \mathrm{m}(\mathrm{n}=5, \mathrm{P}=0.06)$. Both groups showed comparable relative decreases in Dcircle, that is $17.1 \pm 2.4 \%$ for group $\mathrm{A}(\mathrm{n}=2)$ and 18.9 $\pm 11 \%$ for group B $(n=3)$. The mean Dcircle of type II fibres in the control LD muscle measured $63.3 \pm 10.8 \mu \mathrm{m}$. In only 2 goats a few remaining type II fibres were seen, which also demonstrated a decrease in Dcircle (not shown).

Using the Picrosirius-Polarization method, the amount of endomyseal connective tissue appeared to be increased. The area occupied by collagen type I, II and III relative to muscle fibre area increased from $3.2 \pm 2 \%(n=6)$ to $9.0 \pm 4.3 \%(n=5, P<0.05)$.

\section{Electronmicroscopic investigation}

Total mitochondrial volume per muscle fiber increased significantly compared to the control LD muscle. These results are shown in Table 2 demonstrating increases in the peripheral as well as the central part of the stimulated LD muscle fibres. 
56

TABLE 2.

Percentage of mitochondrial volume ( $\%$ mitoch. volume) and of myofibrillar volume (\% myofibr. volumel in the non-stimulated latissimus dorsi muscle and the latissimus dorsi muscle, stimulated for 12 weeks.

\begin{tabular}{lll}
\hline & $\begin{array}{l}\text { \% myofibr. volume } \\
\text { central/peripheral }\end{array}$ & $\begin{array}{l}\text { \% mitoch. volume } \\
\text { central/peripheral }\end{array}$ \\
\hline control LD $(\mathrm{n}=6)$ & $81.7 \pm 6.5 / 81.4 \pm 7.6$ & $0.9 \pm 0.6 / 1.2 \pm 1.1$ \\
stimulated LD 12 weeks $(\mathrm{n}=5$ ) & $73.9 \pm 8.0 / 75.1 \pm 9.4$ & $3.4 \pm 1.7^{*} / 5.3 \pm 1.7^{*}$ \\
\hline
\end{tabular}

The central and the peripheral area of muscle fibers were: used for morphometric analyses.

* significant difference between control and stimulated LD muscle with $\mathrm{P}<0.05$.
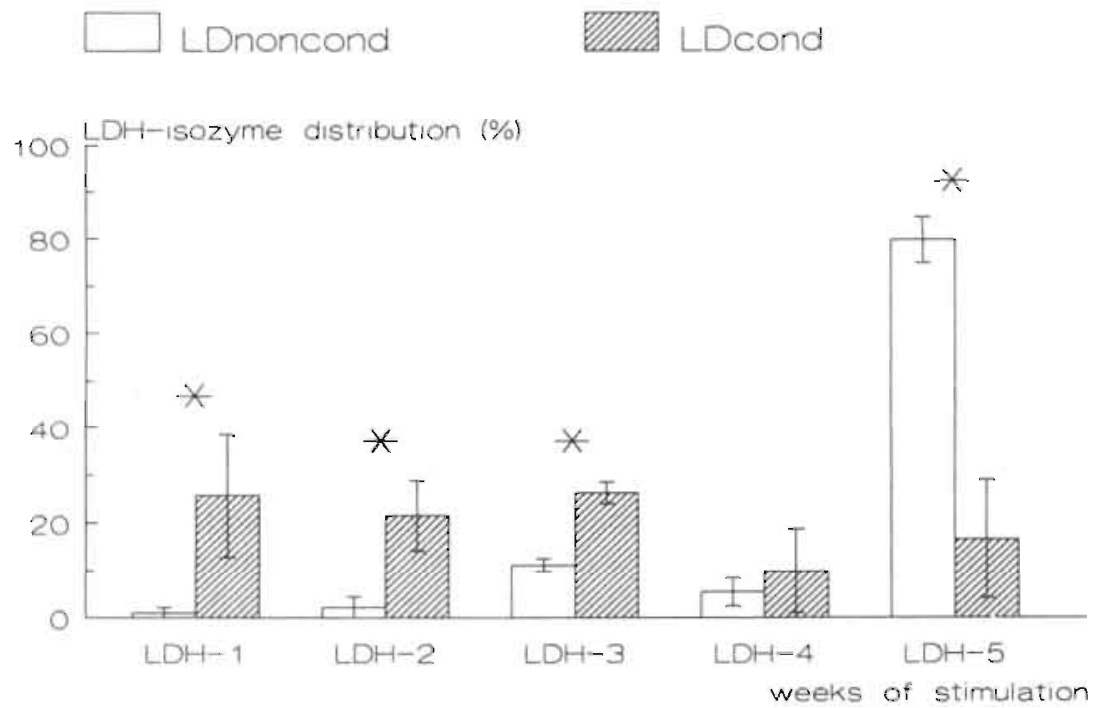

FIGURE 6.

LDH-isozyme distribution presented as the percentage of total LDH activity from conditioned and non conditioned LD muscles. $n=6$ : * Significant difference between both muscles, $P<0.05$.

\section{Enzyme activity measurements}

Stimulation of the LD muscle induced a significant decline in total LDH-activity to $91 \pm 27 \mathrm{U} / \mathrm{gr}$ ww compared to $375 \pm 55 \mathrm{U} / \mathrm{gr}$ ww $(\mathrm{P}<0.001)$ in the non-stimulated muscle. Also CK-activity demonstrated a marked difference between stimulated (1855 $\pm 603 \mathrm{U} / \mathrm{gr}$ ww) and non-stimulated LD muscle (3884 $\pm 574 \mathrm{U} / \mathrm{gr} w \mathrm{w}, \mathrm{P}<0.001)$.

Concomitantly, LDH-isozyme distribution showed an obvious shift from a predominance of LDH type 5 to a significant increase of LDH type 1,2 and 3 (figure 6). 


\section{DISCUSSION}

So far it has been difficult to obtain reliable information about mechanical, structural and biochemical changes in the wrapped LD muscle, following transposition into the thoracic cavity during a cardiomyoplasty procedure. Therefore we used goats in which the LD muscle was stimulated in situ during 12 weeks. Furthermore, in situ stimulation allowed us to compare the mechanical changes which occurred during this period, due to the application of 2 different stimulation protocols.

In previous publications $(14,16,17)$, we presented a model for evaluation of adaptations to conditioning of the LD muscle in the dog. Since then we have used goats instead of dogs for our experiments. Our decision to change to the goat were based upon the greater similarity in blood supply of the human and goat latissimus dorsi muscle as compared to the dog, and the fact that biochemical measurements showed limited adaptations of canine LD muscle to continuous stimulation $(18,19)$, despite concomitant histological changes $(14,20$. ). Few reports about changes in goat LD muscle due to electrical stimulation are available $(3,21)$, in contrast to results obtained in dogs $(19,22-27)$.

In animal experiments, either burst or pulse stimulation is given to the selected skeletal muscle. In clinical practice pacing of the LD muscle in patients is started 2 weeks after surgery with 1 pulse stimulation for a maximum of 50 pulses per minute. In patients, there have been two major reasons to use such a stimulation protocol. First; to prevent early ischemia in the partly dissected LD muscle. Second; a forceful tetanic contraction of the wrapped LD muscle, early after operation, could possibly detach the muscle from the heart. At least two weeks were presumed to be necessary to get optimal adhesion formation between the two muscular layers (3). As demonstrated by our group, it is possible to stimulate the wrapped muscle with two pulses starting already one day after a cardiomyoplasty procedure, if detachment of the muscle from the heart is prevented by the application of fibrin glue $(28,29)$. Therefore, we wanted to compare the currently used clinical protocol with a protocol, immediately beginning with electrical stimulation using a burst of 3 pulses. Our results show that in the goat the clinically used protocol (A) did not lead to the same marked mechanical changes within the first 4 weeks of conditioning as in the 'burst' protocol (B). These differences can be explained by the lesser total number of pulses in protocol A during the early pacing period. Especially a greater increase in fatigue-resistance was obtained after 4 weeks of using protocol B. Because a burst of 3 pulses results in a short tetanic contraction, more muscle force will be produced compared to 1 pulse stimulation. Our observations may be clinically relevant as these patients are in an urgent need of cardiac support after the cardiomyoplasty procedure. Immediate application of a short burst yields more muscle force to assist the failing heart and also results in earlier improvement of fatigue-resistance.

After 12 weeks of stimulation, a significant leftward shift in force-frequency relation was seen, which appeared more pronounced in comparison to the 


\section{8}

results obtained in dogs $(14,17)$. Chachques also reported the important decrease in fusion frequency as a result of electrical stimulation of the LD muscle in goats (2). By programming a burst of $10 \mathrm{~Hz}$ in a trained goat $\mathrm{LD}$ muscle, already $79 \pm 8 \%$ of relative maximal force was attained. In view of these results, it seems sufficient to program a low burst frequency for the wrapped LD muscle in goats after the conditioning period, to get nearly maximal production of force.

Both the literature $(16,18,22,30)$ and our own experience $(14,16)$ indicate that a period of 12 weeks is sufficient to transform the muscle completely to a fatigue-resistant one, mainly composed of type I fibres. In our goats almost complete conversion to type I fibres was seen, comparable to the results of Mannion and coworkers (21).

As demonstrated, a marked increase in fatigue-resistance was obtained after the 12 weeks period of progressive electrical stimulation. According to Bigland-Ritchie, fatigue-resistance depends on the force of the muscular contraction, the duration of contraction and the period in between separate contractions (31). Several factors seem to play a role here. Firstly. the rise in the capillary-to-fibre ratio allows the anatomical substrate sufficient nutritional supply and removal of metabolites in the period in between contractions, as capillary flow is not possible during contraction of the skeletal muscle (32).

Secondly, the oxidative capacity seems to adapt considering the concomitant increase in mitochondrial volume. This finding is underlined by a study from Clark and coworkers. who described an improved metabolic capacity of conditioned canine LD muscle as measured with 31 P-NMR (24). Thirdly, contraction of a slow-twitch muscle, composed of type I fibres is more efficient compared to a fast-twitch muscle mainly composed of type II fibres (33).

Consequently, the stimulated LD muscle appears adapted to perform longterm aerobic work $(25,26)$, for which no extensive glycolytic capacity is needed. The decrease in glycolytic capacity. considering LDH- and CK-activity seems in line with this assumption. Other species like the rabbit and dog demonstrated the same phenomenon $(34,19,14,35,36)$.

The shift in isozyme-pattern of LDH results in a pattern, comparable to the one seen in myocardial muscle fibres, which is in accordance with their need to perform continuous aerobic exercise.

This shift was also observed in other species, following electrical stimulation of skeletal muscle $(14,34,35)$.

Also detrimental effects of continuous electrical stimulation of the goat LD muscle occurred. Although reports have been published which demonstrated a better preservation of skeletal muscle force and muscle fibre diameter, using either burst stimulation $(24,37,38,39,40)$ or a more prudent stimulation protocol (20), we could not demonstrate this in these goat LD muscles. The significant decrease in force, concomitant with the decline in fibre area, could not be prevented by starting muscle stimulation with either 1 or 3 pulses and ending with burst stimulation. Increased stretch of the stimulated muscle, as an additional mechanism to prevent 
muscular atrophy (41), was not tested in these experiments.

As shown, in spite of the achievement of an improved fatigue-resistance, as indicated by better preservation of force during the fatigue test after conditioning of the LD muscle, significant decreases in contraction - (positive $\mathrm{dF} / \mathrm{dt}$ ) and relaxation speed (negative $\mathrm{dF} / \mathrm{dt}$ ) still occurred during the early part of the 8 minutes test. Most investigators consider prolonged preservation of force as a proof of sufficient conditioning of the LD muscle. $(20,22$ 26). As demonstrated by De Haan and coworkers (42), the decrease in velocity of shortening and relaxation rate indicates that fatigue in the conditioned LD muscle obviously still occurs. Particularly relaxation speed appeared seriously affected during the fatigue test. A possible explanation could be the decreased capacity of uptake of $\mathrm{Ca}^{2+}$ by the sarcoplasmic reticulum in a transformed muscle. It is well known that long-term electrical stimulation of a skeletal muscle results in both a pronounced loss in sarcoplasmic reticular volume and a decrease in enzyme-capacity responsible for this transport $(19,36,43,44)$.

Nowadays in clinical use, the fully conditioned wrapped LD muscle is programmed to contract with a maximum of 100 times per minute. Considering the results of our fatigue tests a negative effect, especially on cardiac filling, seems likely when such a high number of contractions is applied. One of our goats even showed severe muscle damage after 12 weeks of continuous stimulation with a maximum of 80 contractions per minute. These observations stress the necessity of more research into the upper rate limit of stimulation of the skeletal muscle used in dynamic cardiomyoplasty. Another possible adverse effect when using skeletal muscle for a dynamic cardiomyoplasty could be the increase in endomyseal connective tissue following electrical stimulation. This phenomenon has been described before in other stimulation experiments $(43,45)$ and can be prevented by stretch of the muscle (46). The increase in connective tissue could result in an increased stiffness of the muscle, as described by Koranen et al (47). They observed this phenomenon in skeletal muscle of humans, participating in endurance training.

\section{In conclusion}

Goat LD muscle does improve fatigue-resistance within 12 weeks of continuous electrical stimulation because of almost complete conversion to type I muscle fibres, an increase in capillary-to-muscle fibre ratio, and a rise in mitochondrial volume. These changes are accompanied by a decrease in fibre area and loss of maximum tetanic force. Also, there is a decrease in glycolytic capacity as shown by a decrease in LDH- and CK-activity. Starting conditioning of the muscle with a short burst of 3 pulses, results in earlier adaptation of the muscle, especially concerning fatigue-resistance. Consequently, application of this stimulation protocol to patients with an LD muscle wrapped around their failing heart, can result in earlier support of cardiac contractile function after cardiomyoplasty.

Our results also suggest the necessity to search for a different stimulation 
60

protocol for the fully conditioned wrapped LD muscle as is used nowadays. Especially the lengthening of the relaxation period of the muscle requires careful determination of the upper rate limit of LD muscle stimulation. 


\section{REFERENCES}

1. Carpentier A. Chachques JC: The use of stimulated skeletal muscle to replace diseased human heart muscle. In: Chiu RC-J (Ed.). Biomechanical cardiac assist: Cardiomyoplasty and muscle-powered devices. Futura Publishing Company. Inc., Mount Kisco, NY, 1986;85-102.

2. Chachques JC, Grandjean PA. Carpentier A: Dynamic Cardiomyoplasty: Experimental Cardiac Wall Replacement with a stimulated skeletal muscle. In: Chiu RC-J (Ed.). Biomechanical cardiac assist: Cardiomyoplasty and musclepowered devices. Futura Publishing Company, Inc. Mount Kisco, NY. 1986;5984.

3. Chachques JC, Grandjean P. Schwartz K, Mihaileanu S, Fardeau M. Swynghedauw B. Fontaliran F, Romero N, Wisnewsky C, Perrier P, Chauvaud S, Bourgeois I, Carpentier A: Effect of Latissimus Dorsi dynamic cardiomyoplasty on ventricular function. Circulation 1988:78 (suppl III):203-216.

4. Magovern G, Heckler F. Park SB, Christlieb IY, Burkholder J, Maher T, Benckart D. Magovern Jr GJ, Kao R: Paced skeletal muscle for dynamic cardiomyoplasty. Ann Thorac Surg 1988;45:614-619.

5. Magovern GJ. Park SB. Kao RL, Christlieb IY, Magovern Jr GJ: Dynamic cardiomyoplasty in patients. J Heart Transplant 1990:9:258-263.

6. National Academy of Sciences: Guide for the Care and Use of Laboratory Animals. DHHS publication No. NIH 85-23. revised 1985.

7. Havenith MG, Visser R. Schrijvers - van Schendel JM, Bosman FT: Muscle fiber typing in routinely processed skeletal muscle with monoclonal antibodies. Histochemistry 1990;93:497-499.

8. Romanul FCA. Bannister R: Localized areas of high alkaline phosphatase activity in the terminal arterial tree. J Cell Biol 1962:15:73-84.

9. Khaw BA. Mattis JA. Melincoff G. Straus HW, Gold HK, Haber E: Monoclonal antibody to cardiac myosin: imaging of experimental myocardial infarction. Hybridoma 1984:3:11-23.

10. Billeter R. Heizmann CW. Howald H, Jenny E: Analysis of myosin light and heavy chain types in single human skeletal muscle fibers. Eur $\mathrm{J}$ Biochem 1981: 1 16:389-395.

11. Staron RS. Pette D: Nonuniform myosin expression along single fibers of chronically stimulated and contralateral rabbit tibialis anterior muscles. Pflügers Arch 1987:409:67-73.

12. Junquira LCU. Montes GS, Sanchez EM: The influence of tissue section thickness on the study of collagen by the picrosirius-Polarization method. Histochemistry 1982:74: 153-156.

13. Dolber PC. Spach MS: Picrosirius red staining of cardiac muscle following phosphomolybdic acid treatment. Stain Technol 1987:62:23-26.

14. Lucas CM. Havenith MG. Van der Veen FH, Habets J. Van der Nagel T, Schrijvers - van Schendel JM. Penn OC. Wellens HJ: Changes in canine latissimus dorsi muscle during 24 weeks of continuous electrical stimulation. J Appl Physiol $1992 ; 72: 828-835$.

15. Dubowitz V: Muscle Biopsy. A practical approach. Second Edition. Balliere Tindall. London. 1982;89. 
16. Havenith MG, Van der Veen FH, Glatz JFC, Lucas C, Schrijvers-van Schendel JMC, Penn OCKM, Wellens HJJ: Monitoring of muscle: fiber type of canine latissimus dorsi muscle during chronic electrical stimulation by enzyme- and immunohistochemistry. In: Chiu RC-J. Bourgeois IM (Eds.). Transformed muscle for cardiac assist and repair. Futura Publishing Company, Inc., Mount Risco, New-York, 1989;53-62.

17. Lucas CMHB, Van der Veen FH, Grandjean PA, Penn OCKM, Wellens HJJ: What is the ideal pulse frequency for skeletal muscle stimulation after cardiomyoplasty. PACE 1991;14: 778-782.

18. Glatz JFC, Van der Vusse GJ, Havenith MG, Van der Veen FH, Lucas CMHB, Penn OCKM, Wellens HJJ: Canine-specific adaptation of energy metabolism of latissimus dorsi muscle in response to chronic electrical stimulation. J Cardiac Surg 1991:6(suppl):265-269.

19. Ianuzzo CD, Hamilton N, O'Brien PJ, Desrosiers C, Chiu R: Biochemical transformation of canine skeletal muscle for use in cardiac-assist devices. J Appl Physiol 1990;68:1481-1485.

20. Kao RL, Trumble DR, Magovern JA, Christlieb IY, Magovern GJ: Fatigue resistant muscle with preserved force and mass for cardiac assist. J Cardiac Surg 1991;6(suppl):210-217.

21. Mannion JD, Shannon J, Chen W, Brown WE, Gale DR: Skeletal muscle-powered assistance for the heart: Assessment of a goat model. In: Chiu RC-J, Bourgeois IM (Eds.). Transformed muscle for cardiac assist and repair. Futura Publishing Company, Inc. Mount Kisco. NY. 1990;117-128.

22. Acker MA, Anderson WA. Hamnond RL, DiMeo F, McCullum J, Staum M, Velchik M, Brown WE. Gale D, Salmons S. Stephenson LW: Oxygen consumption of chronically stimulated skeletal muscle. J Thorac Cardiovasc Surg 1987:94:702709.

23. Chiu RJ-C, Walsh GL, Dewar ML, de Simon J-H, Khalafalla AS, Ianuzzo D: Implantable extra-aortic balloon assist powered by transformed fatigue-resistant skeletal muscle. J Thorac Cardiovasc Surg 1987:94:694-701.

24. Clark BJ, Acker MA, McCully K, Subramanian HV, Hammond RL, Salmons S, Chance B. Stephenson LW: In vivo : 1 P-NMR spectroscopy of chronically stimulated canine skeletal muscle. Am J Physiol 1988;254:C258-C266.

25. Leirner AA, Moreira LFP. Chagas ACP, Cestari LA, Oshiro MS, Nakayama E. da Luz PL. Stolf AG, Jatene AD: Biomechanical circulatory assistance: Importance of aerobic capacity of normal and conditioned skeletal muscle. Trans Am Soc Artif Int Organs 1988;34:716-720.

26. Mannion JD, Bitto T, Hammond RL, Rubinstein NA. Stephenson LW: Histochemical and fatigue characteristics of conditioned canine latissimus dorsi muscle. Cire Res 1987:58:298-304.

27. Walsh GL, Dewar ML. Khalafalla AS, Neilson IR, De Simon J-H, Chiu RC-J: Characteristics of transformed fatigue - resistant skeletal muscle for long-term cardiac assistance by extra-aortic balloon counterpulsation. Surg Forum 1986; 37:205-207.

28. Lorusso R, Lucas C, Van $\operatorname{der}$ Veen F. Habets J. Van der Nagel T, Havenith M, Wellens HJ, Penn OC: Early Latissimus Dorsi stimulation after cardiomyoplasty procedure: A preliminary study. BAM 1991:1:83-88.

29. Lorusso R. Lucas C, Van der Veen F, Habets J, Van der Nagel T, Havenith M. Wellens HJ. Penn OC: Fibrin sealant application during dynamic cardiomyoplasty. In: DAllessandro LC (Ed.). Heart Surgery 1991. Casa Editrice Scientifica Internationale. 1991:579-584. 
30. Acker MA. Hammond RL. Mannion JD, Salmons, S, Stephenson LW: An autologous biologic pump motor. J Thorac Cardiovasc: Surg 1986;92:733-74.6.

31. Bigland-Ritchie B, Cafarelli E, Vollestad NK: Fatigue of submaximal contractions. Acta Physiol Scand 128:(suppl556): 137-148.

32. Quensel W. Kramer K: Untersuchungen uber den Muskelstoffwechsel des Warmbluters. II. Die Sauerstoffaufnahme des Muskels wahrend der tetanische Kontraction. Pflügers Arch ges Physiol 1939;241:698-716.

33. Crow MT, Kushmerick MW: Chemical energetics of slow- and fast-twitch muscles of the mouse. J Gen Physiol 1982;79:147-166.

34. Henriksson J, Chi M-Y, Hintz CS, Young DA, Kaiser KK, Salmons S, Lowry OH: Chronic stimulation of mammalian muscle: Changes in enzymes of six metabolic pathways. Am J Physiol 1986:251:C614-C632.

35. Pette D. Smith ME, Staudte HW, Vrbova G: Effects of long-term electrical stimulation on some contractile and metabolic characteristics of fast rabbit muscles. Pflügers Arch 1973:338:257-272.

36. Reichmann H. Hoppeler H, Mathieu-Costello O, von Bergen F, Pette D: Biochemical and ultrastructural changes of skeletal muscle mitochondria after chronic electrical stimulation in rabbits. Pflügers Arch 1985;404:1-9.

37. Brown MD, Cotter MA, Hudlicka O, Vrbova G: The effects of different patterns of muscle activity on capillary density, mechanical properties and structure of slow and fast rabbit muscles. Pflügers Arch 1976;361:241-250.

38. Eerbeek O, Kernell D. Verhey B: Effects of fast and slow patterns of tonic long-term stimulation on contractile properties of fast muscle in the cat. J Physiol 1984:352:73-90.

39. Ferguson AS. Stone HE. Roessmann U, Burke M, Tisdale E, Mortimer JT: Muscle plasticity: Comparison of a $30-\mathrm{H} /$ burst with $10-\mathrm{Hz}$ continuous stimulation. J Appl Physiol 1989:66:1143-1151.

40. Jarvis JC, Mayne CN. Salmons S: Basic studies on skeletal muscle for cardiac assistance. J Cardiac Surg 1991;6(suppl):204-209.

41. Goldspink DF. Easton J. Winterburn SK. Williams PE. Goldspink GE: The role of passive stretch and repetitive electrical stimulation in preventing skeletal muscle atrophy while reprogramming gene expression to improve fatigue resistance. J Cardiac Surg 1991;6(suppl):218-224.

42. De Haan A. Jones DA. Sargeant AJ: Changes in velocity of shortening, power output and relaxation rate during fatigue of rat medial gastrocnemius muscle. Pflügers Arch 1989:413:422-428.

43. Eisenberg BR, Salmons S: The reorganization of subcellular structure in muscle undergoing fast-to-slow type transformation. Cell Tiss Res 1984;220:22 1-230.

44. Klug G, Wiehrer W. Reichmann H. Leberer E, Pette D: Relationships between early alterations in parvalbumins, sarcoplasmatic reticulum and metabolic enzymes in chronically stimulated fast twitch muscle. Pflügers Arch 1983; 399:280-284.

45. Mannion JD. Velchik M. Hammond R, Alavi A, Mackler T, Ducketı S, Staum M, Hurwitz S, Brown W, Stephenson LW: Effects of collateral blood vessel ligation and electrical conditioning on blood flow in dog Latissimus Dorsi muscle. J Surg Res 1989;47:332-340.

46. Williams PE, Catanese T, Lucey EG: The importance of stretch and contractility in preventing connective tissue accumulation in muscle. J Anat 1988;158:109. 114.

47. Koranen V. Suominen H. Heikkinen E: Mechanical properties of fast and slow skeletal muscle with special reference to collagen and endurance training. J Biochem 1984; 17:725-735. 
hapter 4

\section{Imipramine induced heart failure in the dog:}

\section{A model to study the effect of cardiac assist devices}

Caroline MHB Lucas

Emile C Cheriex

Frederik $H$ van der Veen

Jo Habets

Theo van der Nagel

Olaf C Penn

Hein $\mathrm{J}$ Wellens

Abstract published in The FASEB Journal 1990:4:757A.

Accepted for publication in Cardiovasc Res. 


\title{
Imipramine induced heart failure in the dog:
}

\section{A model to study the effect of cardiac assist devices}

\begin{abstract}
The value of intravenous imipramine administration to create a reversible model of short-term heart failure was evaluated in anesthetized dogs. Acute effects of imipramine were studied in 11 dogs using invasive hemodynamic pressure measurements and two-dimensional echo evaluation. After 30 minutes of imipramine in a dosage of $7.5 \mathrm{mg} / \mathrm{kg} /$ hour. positive left ventricular $\mathrm{dP} / \mathrm{dtmax}$ decreased from $1368 \pm 108 \mathrm{mmHg} /$ $\mathrm{sec}$ (mean $\pm \mathrm{SE}$ ) to $909 \pm 119 \mathrm{mmHg} / \mathrm{sec}(\mathrm{P}<0.05)$, left ventricular end-diastolic pressure increased from $8 \pm 1 \mathrm{mmHg}$ to $12 \pm 2 \mathrm{mmHg}(\mathrm{P}<$ 0.05 ), while left ventricular pressure decreased from $106 \pm 4 \mathrm{mmHg}$ to $87 \pm 6 \mathrm{mmHg}(P<0.05)$. Cessation of imipramine administration resulted within 60 minutes in partial restoration of cardiac function. This deterioration and subsequent recovery was also demonstrated with echocardiographic measurements demonstrating a decrease in ejection fraction from $54 \pm 3$ to $28 \pm 2 \%(P<0.05)$. During administration of imipramine neither significant electrophysiological changes nor supraventricular and ventricular arrhythmias were seen. Repeated infusions of imipramine in three anesthetized dogs with an interval of two weeks showed the reproducibility of the hemodynamic effects and the recovery of ventricular function. Because we developed our model with the intention to evaluate the use of cardiomyoplasty in heart failure, the effect of imipramine was also evaluated on contractile force of the latissimus dorsi muscle. Force measurements of the left latissimus dorsi muscle were performed by connecting the left forefoot to a Force Displacement Transducer. It was found that administration of imipramine did not affect skeletal muscle force development at the dosage used to create heart failure. Our results suggest the use of this model
\end{abstract}


to produce short-term reversible heart failure in anesthetized animals to test the efficacy of supportive interventions like dynamic cardiomyoplasty, intra-aortic balloon pumping and mechanical cardiac assist devices.

\section{INTRODUCTION}

To test the effectiveness of new pharmacological or mechanical modes of therapy to help patients with heart failure, one would like to have a reliable failing heart model in a large animal. However, experimental induction of a failing heart in large animals is frequently complicated by a high incidence of cardiac death or chronic injury to other organs (1). Furthermore, because we wanted to study the effect of biomechanical interventions, like the use of skeletal muscle as in dynamic cardiomyoplasty or muscle-powered devices in patients with severe chronic cardiac failure (2-9), a model is required without adverse effects on skeletal muscle contractility.

In this article we would like to report on our experience with the intravenous use of imipramine, a tricyclic antidepressive drug, to create reversible short-term cardiac failure in the anesthetized dog. This model appears to be a promising new way to repeatedly study the effect of non-pharmacological interventions to treat cardiac failure.

\section{MATERIALS AND METHODS}

\section{Animal preparation}

The experiments were done in 11 mongrel dogs of either sex, weighing 25 to $38 \mathrm{~kg}$. All experiments were performed in accordance with the 'Guide for the care and Use of Laboratory Animals' prepared by the National Academy of Sciences (10). The animals were premedicated with a drug mixture $(0.2$ $\mathrm{ml} / \mathrm{kg}$ ) intramuscularly. One cc of this mixture consisted of $10 \mathrm{mg}$ oxycodon HCL, $1 \mathrm{mg}$ acepromazine and $0.5 \mathrm{mg}$ atropinesulphate. Anesthesia was induced by giving sodium pentobarbital (Narcovet) intravenously in a dose of $10 \mathrm{mg} / \mathrm{kg}$. Following endotracheal intubation, anesthesia was maintained with oxygen/nitrous oxide and Fluothane $(0.5 \%-1 \%)$. The lungs were ventilated with a positive pressure respirator (Pulmomat). During the experiments, body temperature was kept constant with a heating blanket. Arterial blood gasses were measured routinely to insure adequate anesthesia and ventilation. Preparation was performed under sterile conditions and antibiotics were given immediately preoperatively and for 2 days post-operatively (Amfipen $1000 \mathrm{mg}$ iv and im). A braunule was inserted in the right brachial vein for continuous infusion of $0.9 \%$ saline at a rate of $3 \mathrm{cc}$ per minute. The electrocardiogram was recorded using the limb leads lleads I, II and III). Left ventricular systolic pressure measurements were obtained with a Millar catheter-tip micromanometer (7F Millar, PC470) introduced by way of the left brachial artery. Left ventricular end-diastolic pressure was 
separately recorded by clipping the left ventricular pressure at $20 \mathrm{mmHg}$ and subsequently amplifying the signal on the recorder. The first derivative (positive and negative left ventricular $\mathrm{dP} / \mathrm{dtmax}$ ) was determined as described by Schaper et al (11). Right ventricular systolic pressure was recorded by a polyethylene catheter connected to an Ailtech pressure transducer, introduced in the right ventricle by way of the right jugular vein. The catheters were kept patent by flushing them repeatedly with saline and heparin. All pressure transducers were calibrated before infusion of imipramine and subsequently every 15 minutes during the experiment. Echocardiographic measurements of the effect of imipramine infusion were made on a Hewlett-Packard $77020 \mathrm{~A}(\mathrm{Cl})$ echocardiograph, using a $5 \mathrm{Mhz}$ medium focused phased array transducer. A right parasternal approach was used during the investigation. This allows a classical short axis view of the left ventricle. Both two dimensional and M-mode echocardiographic recordings were made. Left ventricular dimensions were measured using the ASE recommendations (12). Left ventricular volumes were calculated according to the formula of Teichholtz (13).

\section{Induction of cardiac failure}

Thirty minutes after the insertion of the pressure catheters, with the dog in a hemodynamically stable condition, the intravenous imipramine infusion was started. Electrocardiogram, right ventricular systolic pressure, left ventricular systolic pressure, left ventricular end-diastolic pressure, and positive $\mathrm{dP} / \mathrm{dtmax}$ of the left ventricle were continuously monitored thereafter. Imipramine (Tofranil, Ciba-Geigy, AG, Basel, Switzerland) was infused in a dosage of $7.5 \mathrm{mg} / \mathrm{kg} /$ hour through the braunule in the right brachial vein. Every 10 minutes echocardiographic views were obtained. During the infusion the ECG was continuously observed for rhythm disturbances, widening of the QRS-complex, changes in QT-interval and repolarization disturbances because these changes have been reported after an overdose of a tricyclic antidepressive drug (14-16,17,37). The infusion was stopped when either the positive left ventricular $\mathrm{dP} / \mathrm{dtmax}$ had decreased to less than $600 \mathrm{mmHg} / \mathrm{sec}$ or left ventricular end-diastolic pressure had increased above $20 \mathrm{mmHg}$. If these values were not reached, the infusion was stopped after one hour. All above mentioned variables were continuously monitored during one hour after the cessation of the imipramine infusion. Effects of discontinuation of imipramine was also evaluated by echocardiography. In 3 dogs the same protocol was repeated twice with an interval of 2 weeks in between the infusions, to study the reproducibility and possible long-term effects of imipramine. In one of these dogs, echocardiographic measurements were repeated three times.

\section{Skeletal muscle preparation}

In 3 dogs, prior to imipramine infusion and in a separate operation, 2 intramuscular electrodes (Medtronic SP5528) were implanted in the proximal part of the left latissimus dorsi (LD) muscle, perpendicular to the main branches of the nervus thoracodorsalis, and subsequently connected to an 
Itrel pacemaker (Medtronic SP7420). These dogs were positioned on their right side and connected with their left forefoot to a force displacement transducer (Grass FT 10, Grass Instruments, Quincy, Mass. USA), to measure isometric force of the left $\mathrm{LD}$ muscle as previously described by our group (18). Thereafter the infusion of innipramine, as described above, was performed. The LD muscle force in these dogs was measured by stimulating the LD muscle with a $0.25 \mathrm{sec}$ burst of pulses with a burst frequency of 30 $\mathrm{Hz}$ and an individual pulse width of $210 \mu \mathrm{sec}$. Ten bursts at a supramaximal voltage of 5 volt were recorded with a pause between two bursts of $10 \mathrm{sec}$. Skeletal muscle stimulation was performed at the beginning and at the end of the imipramine infusion.

All measurements were recorded on a multichannel Schwarzer recorder continuously at a paper speed of $1.25 \mathrm{~mm} / \mathrm{sec}$ and every 5 minutes at 25 and $200 \mathrm{~mm} / \mathrm{sec}$.

\section{Statistical analysis}

The data were compared statistically using Wilcoxon's test for paired samples. Differences were interpreted as being statistically significant at $P$ $<0.05$. Values are presented as mean $\pm \mathrm{SE}$.

\section{RESULTS}

\section{Hemodynamic effects}

In all 11 dogs a steady decline in left ventricular pressure and left ventricular $\mathrm{dP} / \mathrm{dtmax}$ was observed starting immediately after the start of imipramine infusion (figures $1 \mathrm{a}$ and $\mathrm{lb}$ ). This resulted in a left ventricular pressure of $87 \pm 6 \mathrm{mmHg}$ and a left ventricular $\mathrm{dP} / \mathrm{dt}$ of $909 \pm 119$ $\mathrm{mmHg} / \mathrm{sec}$ after 30 minutes of intravenous administration. During this time period left ventricular end-diastolic pressure increased from $8 \pm 1$ to $12 \pm 2$ $\mathrm{mmHg}$ (figure $1 \mathrm{c}$ ). Concomitantly, right ventricular pressure demonstrated a decrease in pressure from $24 \pm 2$ to $20 \pm 1 \mathrm{mmHg}$.

Using the criteria described in the method section, imipramine infusion was terminated after 30 to 60 minutes with a mean duration of $50 \pm 3$ minutes.

Figure 1 also illustrates the effect of cessation of imipramine infusion on left ventricular pressure, positive left ventricular $\mathrm{dP} / \mathrm{d} t \max$ and left ventricular end-diastolic pressure. Both left ventricular pressure (figure la) and positive left ventricular $\mathrm{dP} / \mathrm{dtmax}$ (figure $\mathrm{lb}$ ) showed significant restoration of values after 15 minutes. Left ventricular end-diastolic pressure however, did not improve significantly before 60 minutes after the end of the imi-pramine infusion (figure 1c). In Table 1 the results of all pressure measurements are summarized. Although start of restoration of these values was observed following cessation of imipramine. complete recovery did not occur within 60 minutes after termination of imipramine administration. This is understandable in view of the 'half life' of elimination ( \pm 35 hours) (19). The effect 
LVP

a

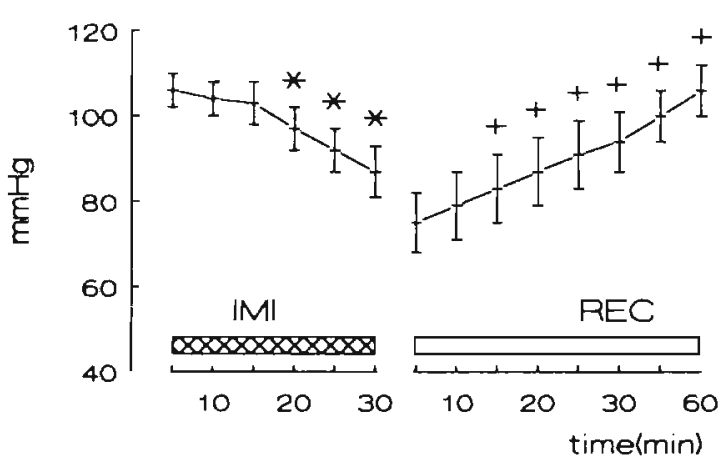

$\llcorner\vee d P / d t m a x$

b

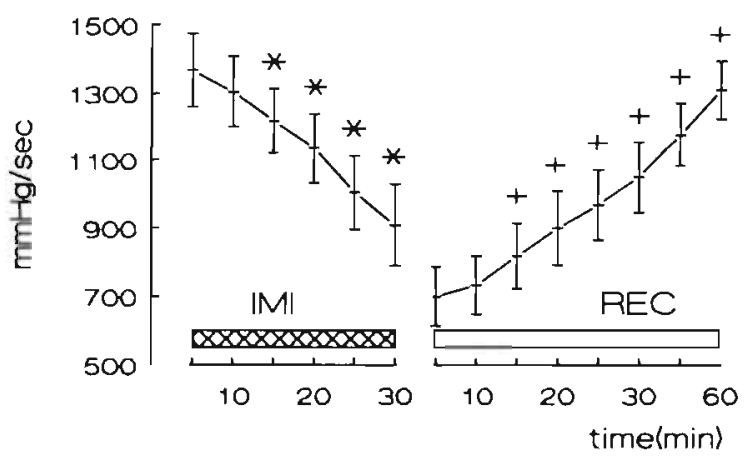

LVEdP

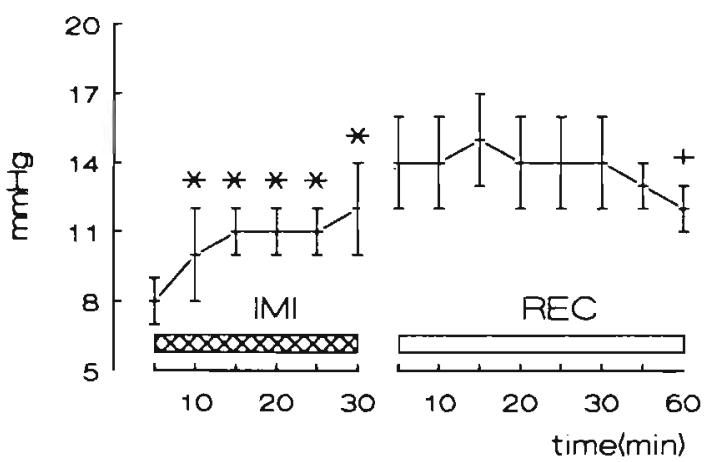

FIGURE 1.

Left ventricular systolic pressure (LVP. la), left ventricular $d P / d t \max (L V d P / d t m a x, l b)$ and left ventricular end-diastolic. pressure (LVEdP. IC) measured during the first 30 minutes of imipramine infusion and during 60 minutes after its cessation. IMI: period of imipramine infusion. $R E C$ : period of recovenj after imipramine infusion values are presented as the mean $\pm S E$ of 11 dogs $(*: P<0.05$ comparing imipramine with control values. $+: P<0.05$ comparing data after cessation of imipramine with values at end of infusion). 
TABLE 1.

Invasive hemodynamic measurements before, at the end of umipramine infusion and one hour later.

\begin{tabular}{llll}
\hline & $\begin{array}{l}\text { Before } \\
\text { imipramine }\end{array}$ & End of infusion & one hour recovery \\
\hline LVP (mmHg) & $106 \pm 4$ & $87 \pm 6^{*}$ & $103 \pm 6^{+}$ \\
LVdP/dtmax (mmHg/sec) & $1368 \pm 108$ & $909 \pm 119^{*}$ & $1298 \pm 92^{*}$ \\
LVEdP (mmHg) & $8 \pm 1$ & $12 \pm 2^{*}$ & $12 \pm 1^{+*}$ \\
\hline
\end{tabular}

Abbreviations: $L V P=$ Left ventricular pressure: $L V d P / d t m a x=L e f t$ ventricular $\mathrm{dP} / \mathrm{dt}$ max: LVEdP = Left ventricular end-diastolic pressure. Values are presented as the mean $\pm \mathrm{SE}$ : *:P $<0.05$ comparing imipramine with control values, $+: \mathrm{P}<0.05$ comparing data at the end of the imipramine infusion and one hour later.

TABLE 2.

Echocardiographic measurements before, at the end of imipramine infusion and one hour later.

\begin{tabular}{llll}
\hline & Before imipramine & End of infusion & one hour recovery \\
\hline LVEDD (mm) & $39 \pm 3$ & $48 \pm 0.4^{*}$ & $45 \pm 1.5$ \\
LVESD (mm) & $29 \pm 3$ & $42 \pm 0.5^{*}$ & $32 \pm 2.5^{+}$ \\
LVEDV (ml) \# & $68 \pm 10$ & $108 \pm 2^{*}$ & $92 \pm 7^{+}$ \\
LVESV (ml)\# & $32 \pm 8$ & $78 \pm 2^{*}$ & $42 \pm 7^{+}$ \\
LVEF (\%) & $54 \pm 3$ & $28 \pm 2^{*}$ & $55 \pm 5^{+}$ \\
\hline
\end{tabular}

Abbreviations: $L V E D D=$ Left ventricular end-diastolic dimension: LVESD $=$ Left ventricular end-systolic dimension; LVEDV = Left ventricular end-diastolic volume: LVESV = Left ventricular end-systolic volume: $L V E F=$ Left ventricular ejection fraction. Values are presented as the mean $\pm \mathrm{SE}:{ }^{*}: \mathrm{P}<0.05$ comparing impramine with control values, $+: P<0.05$ comparing data at the end of the imipramine infusion and one: hour later. \#: Calculated according to the formula of Teichholtz (13).

of imipramine infusion and subsequent withdrawal on cardiac function is also demonstrated by the echocardiographic data shown in Table 2. As shown infusion of this drug resulted in pronounced increases both in left ventricular end-systolic and end-diastolic volume, concomitant with a decrease in left ventricular ejection fraction. Withdrawal of the drug also demonstrated partial recovery of these values within one hour.

The hemodynamic effect of repeated doses of imipramine with an interval of two weeks is shown in figure 2 , in which the mean values of three dogs are presented during 3 consecutive infusions. During all doses reproducible decreases in left ventricular pressure and positive left ventricular dP/ dtmax, concomitant with increases in left ventricular end-diastolic pressure were seen within 30 minutes. Discontinuation of imipramine infusion resulted in marked improvement of cardiac contractility as reflected by increases in left ventricular pressure and positive left ventricular $\mathrm{dP} / \mathrm{dtmax}$ within 60 minutes during all three sessions (figures $2 a$ and $2 b$ ). The left ventricular end-diastolic pressure behaved less consistent (figure $2 \mathrm{c}$ ). To 


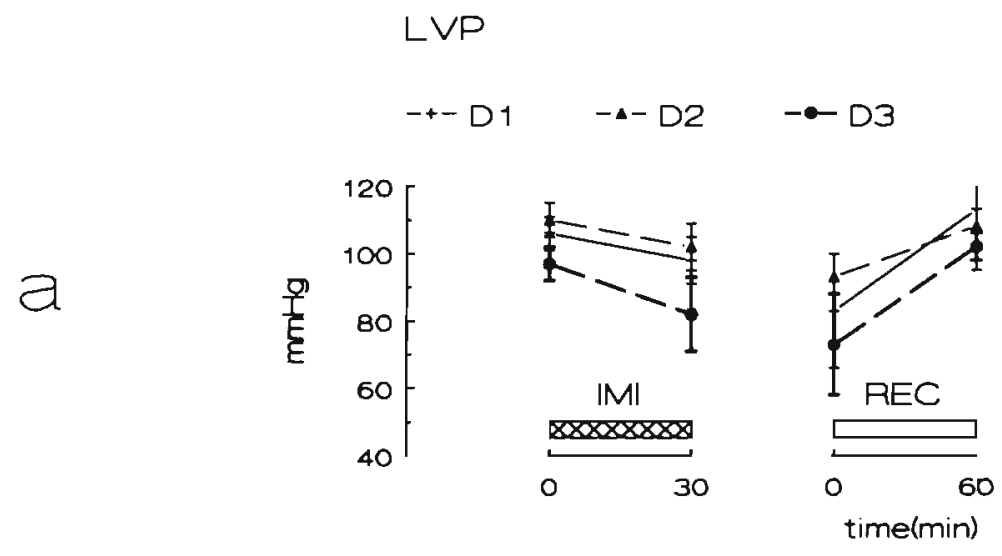

\section{LVdP/dtmax}

0

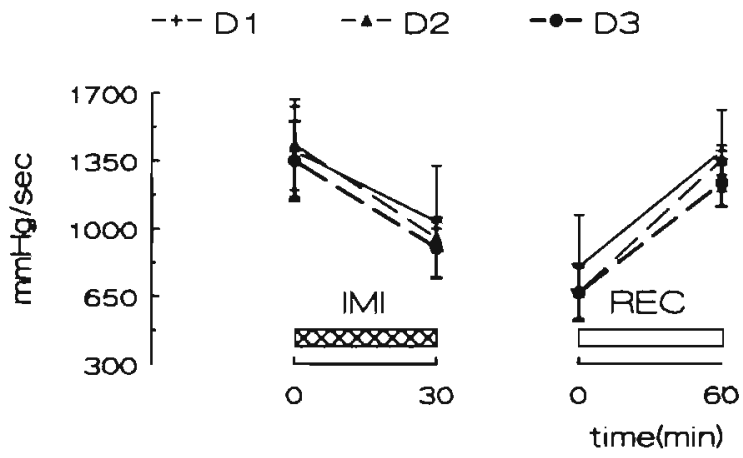

LVEdP

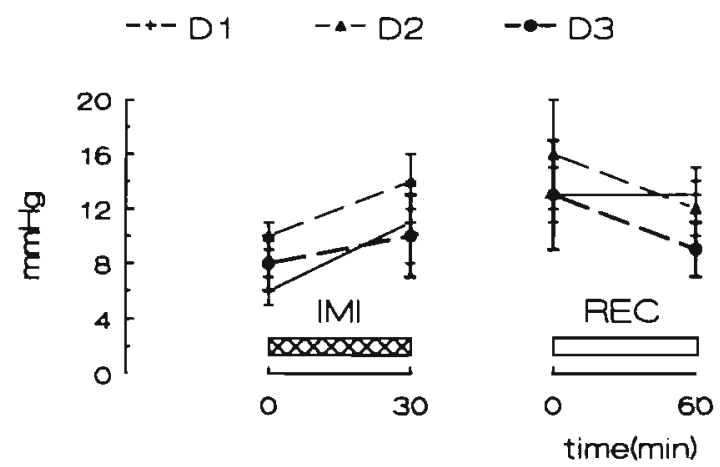

FIGURE 2.

Left ventricular pressure (2a). left ventricular positive $d P / d t m a x(2 b)$ and left ventricular end-diastolic pressure (2c) represented as mean values of three dogs during three repeated episodes of imipramine administration at two weeks intervals. A similar way of presentation of data is selected as in figure 1. D1, D2 and D3 describe the consecutive doses. 


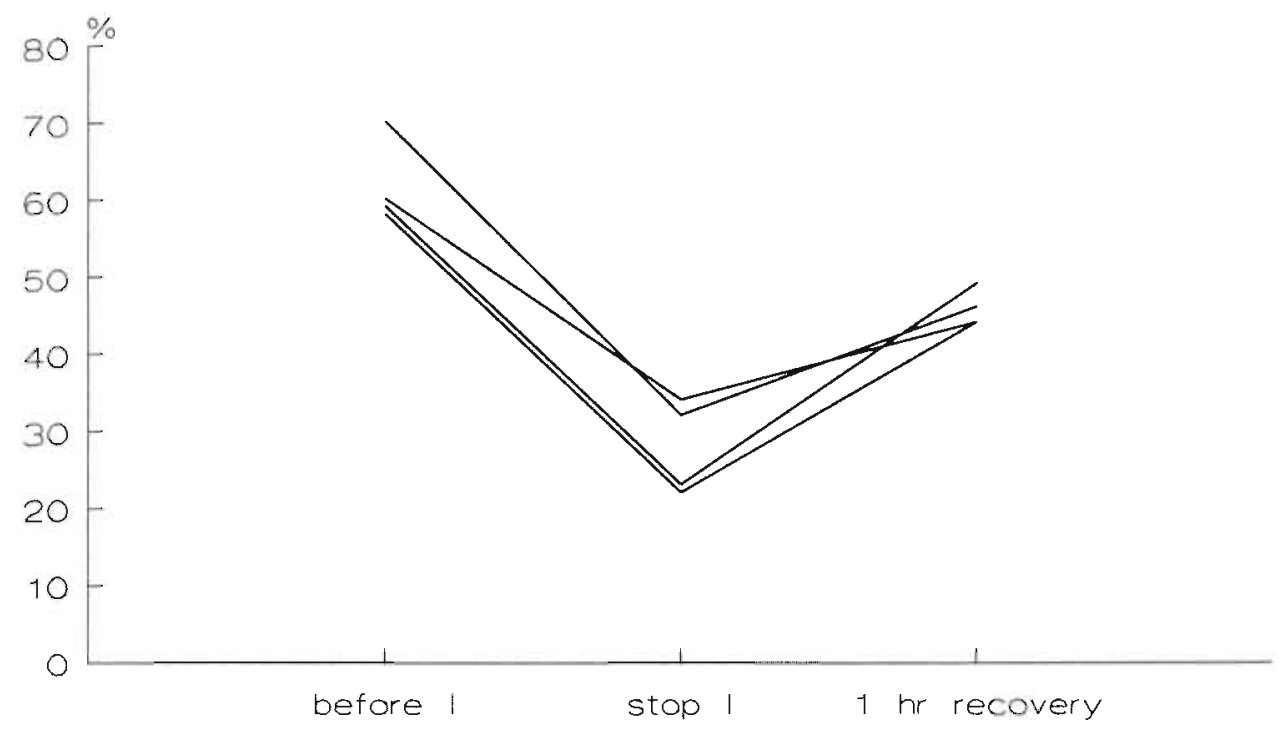

FIGURE 3.

Left ventricular ejection fraction before, during and one hour after the cessation of imipramine administration during four repeated doses in one dog.

demonstrate the complete recovery and the reproducibility of this model the behavior of left ventricular ejection fraction is pictured in figure 3 during 4 repeated doses of imipramine in one dog.

Electrophysiologic effects

Both before, during and after the period of infusion of imipramine a sinustachycardia was observed in all dogs without significant changes in heart rate ( $133 \pm 6$ at the beginning vs $126 \pm 7$ beats per minute at the end of the infusion). No supraventricular nor ventricular arrhythmias were observed. A small but significant increase in width of the QRS-complex was observed from $44 \pm 2 \mathrm{msec}$ to $48 \pm 2 \mathrm{msec}(\mathrm{P}<0.05)$ concomitant with a non-significant increase in $\mathrm{QTc}$ from $310 \pm 8 \mathrm{msec}$ to $314 \pm 3 \mathrm{msec}$. Furthermore, some dogs showed a reversible $\mathrm{T}$-wave inversion.

Skeletal muscle force

In the dogs with the LD muscle preparation no effect was seen on muscle force, both during infusion and subsequent withdrawal of the drug. To underline this phenomenon, an example of LD muscle force measurements concomitant with pressure measurements during infusion of imipramine is pictured in figure 4.

All animals were vital and eating normally the day after the experimental protocol was completed and no complications were observed during a follow-up period of at least one month in all dogs. 

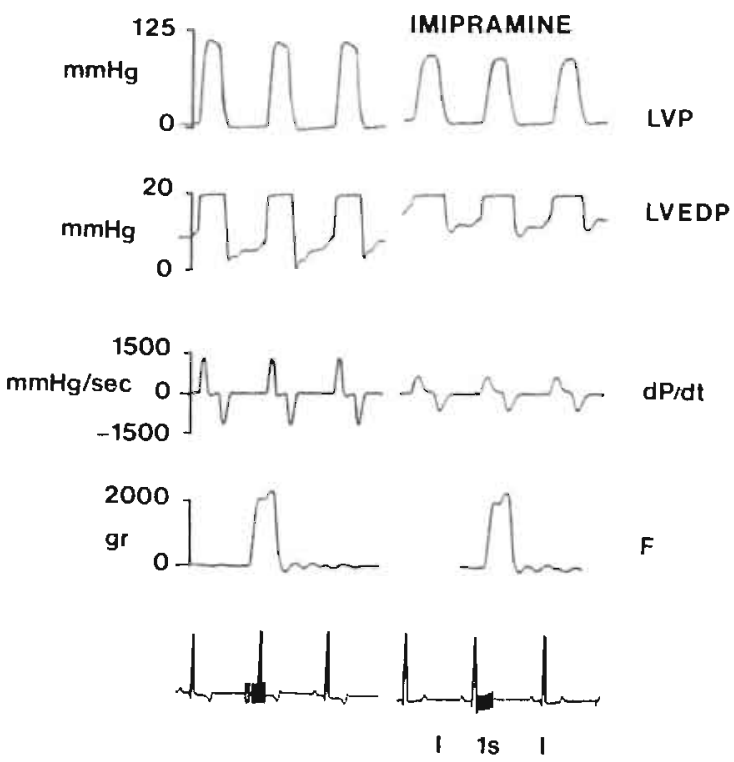

\section{FIGURE 4.}

Recordings showing hemodynamic measurements together with force measurements of the left $L D$ muscle before lleft panel) and after (right panel) imipramine infusion. (LVP = left ventricular pressure, $L V E d P=$ left ventricular end-diastolic pressure. $d P / d t=d P / d t$ of the left ventricle, $F=$ force of the left $L D$ muscle). The electrocardiogram-tracing demorustrates a train of spikes reflecting electrical actication of the $L D$ muscle. In contrast to the effect on cardiac contractility imipramine does not affect force of the left $L D$ muscle.

\section{DISCUSSION}

Several methods to create a failing heart model have been presented in the literature, including a discussion on their numerous limitations (1). Induction of a failing heart following coronary artery occlusion using a diversity of methods has been reported. However, this rarely resulted in stable cardiac failure. because dogs either compensated for the reduction of viable myocardial tissue or died due to rhythm disturbances or cardiogenic shock (20-24). In particular the large variations in collateral coronary circulation (25) made it difficult to obtain reproducible and stable cardiac failure. The use of cardiotoxic drugs such as adriamycine (26) or substances as cobalt (27) to induce a failing heart, resulted in severe multi-organ injury. In order to study cardiac assist after cardiomyoplasty, a reproducible failing heart model with low morbidity and mortality is required. Moreover, in these animals skeletal muscle contractility should not be affected. 
Van de Ree and coworkers $(14,28)$ reported on the detrimental effect of imipramine, a tricyclic antidepressive drug, on cardiac function. These investigators studied the possible modes of action on cardiac contractility using relatively high dosages. Partially successful reversal of the effects of intoxication of imipramine by dopamine and isoproterenol administration were described by Sangster et al (29).

These observations prompted us to examine the use of imipramine to produce a failing heart in anesthetized dogs with a high degree of reproducibility and a low risk of death, both acutely and long-term. In addition, in vivo information on the effect of imipramine infusion on latissimus dorsi muscle contractility was required in order to decide whether this falling heart model could be used to study the efficacy of skeletal muscle assistance as, for example, in cardiomyoplasty in animals.

Our findings confirm previous experiments by Van de Ree et al $(14,28)$ on the cardiodepressive effect of imipramine. The exact mechanism by which this drug exerts its detrimental effect on cardiac function still remains to be elucidated. So far several different pharmacological effects have been described (16-18). Imipramine; 1) Has an anticholinergic action; 2) Blocks re-uptake of norepinephrine at the side of the adrenergic neurons; 3) Exerts a frequency- and voltage-dependent inhibition of fast sodium channels in the myocardium resulting in a decrease in Vmax. (class Ib-antiarrhythmic action) (30.31) 4) Influences the $\mathrm{Ca}^{2+}$-handling during the action potential in myocardial cells as demonstrated by a reduction of inward $\mathrm{Ca}^{2+}$-current during phase $2\left(\mathrm{ICa}^{2+}\right.$ ) (31). This effect is also underlined by the beneficial anti-arrhythmic effect of this drug during ouabain intoxication which results in a $\mathrm{Ca}^{2+}$ overload in myocytes (32). In particular this decrease in $\mathrm{ICa}^{2+}$ could be responsible for the loss of cardiac contractility as suggested by Isenberg et al (31); 5) Blocks the peripheral $\alpha$-adrenergic receptors. All our dogs responded with a decrease in cardiac contractility as reflected in the positive left ventricular $\mathrm{dP} / \mathrm{dtmax}$ and left ventricular ejection fraction values, although there was some variation in time interval until this decrease reached its maximal value. These variables can be used as reliable indices of contractility (33), because both no significant change in heart rate occurred during the infusion of imipramine and no significant change in peripheral resistance as demonstrated by Van de Ree et al (14) and Nattel and coworkers (34). Moreover, peripheral reflexes are maintained adequately (14) which is not the case with for example an overdose of barbiturate (35). A pronounced depression of left ventricular function can be obtained up to a value of a positive left ventricular $\mathrm{dP} / \mathrm{dtmax}$ of $\pm 600 \mathrm{mmHg} / \mathrm{sec}$ without, significant changes in the QRS-complex or QT-interval as shown in our experiments. Also, no arrhythmias were observed. Continuous monitoring of the ECG should be performed however, because a relationship between the width of the GRS-complex and the appearance of arrhythmias has been described, especially if this value exceeds $100 \mathrm{msec}(15-17)$. Nattel and coworkers also demonstrated that a pronounced increase in QRS-width occurred immediately before the development of ventricular tachyarrhythmias (36). In our opinion this value seems to be useful as a marker to stop 
administration of imipramine during the induction of a failing heart. This is underlined by the fact that cessation of drug infusion results in rapid and complete restoration of myocardial function thus indicating the safety of this model. Also, the reversibility of effects after repeated doses in the same dog indicate the possibility to use this model repeatedly to test different models of cardiac assistance. Unfortunately, this drug is only suitable to create a failing heart in anesthetized animals as serious symptoms of central nervous system toxicity have been described in awake dogs [36].

To summarize: Several factors favor the use of imipramine to obtain a short-term failing heart model in anesthetized animals. First, it is an easily controllable, reproducible and safe method with no risk of acute or longterm mortality. Also, the negative action of imipramine on cardiac contractility is visible within a short period of time and its administration can be repeated several times without any unpleasant side effects for the dogs. The animals do not need special care in the period in between the infusions of repeated doses of imipramine, as is the case with the use of cardiotoxic drugs or a diet deficient in an essential element $(26,37)$. Although this model will not be appropriate to study the effect of positive inotropic support of drugs on depressed myocardial function, it is very well suited to test the effect of intra-aortic devices, mechanical devices or skeletal muscle to assist the failing heart, especially because skeletal muscle force is not affected by imipramine. 


\section{REFERENCES}

1. Smith HJ, Nuttall A: Experimental Models of heart failure. Cardiovasc Res $1985 ; 19: 181-186$.

2. Anderson DR, Pochettino A, Hammond RL, Hohenhaus E. Spanta AD, Bridges CR, Lavine S, Bhan RJ, Colson M, Stephenson LW: Autogenously lined skeletal muscle ventricles in circulation: Up to nine months' experience. J Thorac Cardiovasc Surg 1991;101:661-670.

3. Kao RL, Christlieb IY, Magovern GJ, Park SB, Magovern Jr GJ: The importance of skeletal muscle fiber orientation for dynamic cardiomyoplasty. J Thorac Cardiovasc Surg 1990;99:134-140.

4. Chachques JC. Grandjean P. Schwartz K: Effect of latissimus dorsi dynamic cardiomyoplasty on ventricular function. Circulation 1988;78(suppIII):203-16.

5. Anderson WA, Andersen JS. Acker MA: Skeletal muscle grafts applied to the heart: A word of caution. Circulation 1988;78(suppIII):180-190.

6. Li CM, Hill A, Colson M. Desrosiers C, Chiu RC-J: Implantable rate-responsive counterpulsation assist system. Ann Thorac Surg 1990;49:356-362.

7. Nilnami H, Hooper TL, Hammond RL: A new configuration for right ventricular assist with skeletal muscle ventricle: Short-term studies. Circulation 1991; 84:2470-2475.

8. Kochamba G, Desrosiers C, Dewar M, Chiu RC-J: The muscle-powered dualchamber counterpulsator: Rheologically superior implantable cardiac assist device. Ann Thorac Surg 1988:45:620-625.

9. Anderson WA, Bridges CR, Chin AJ: Long-term neurostimulation of skeletal muscle: Its potential for a tether-free biologic cardiac assist device. PACE $1988 ; 11 ; 2128-2134$.

10. National Academy of Sciences. Guide for the care and use of laboratory animals. DHHS publication No NIH 85-23, revised 1985.

11. Schaper W, Lewi P. Jageneau AHM: The determination of the rate of change of the left ventricular pressure (dP/dt). Arch Kreislaufforsch 1965;46:27-41.

12. Sahn DJ, DeMaria A, Kisslo J, Weyman A: Recommendations regarding quantitation in M-mode echocardiography; Results of a survey of echocardiographic measurements. Circulation 1978:58:1072-1083.

13. Teichholtz LE, Kreulen P. Herman MC, Gorlin R: Problems in echocardiographic volume determination: Echocardiographic - angiographic correlations in the presence or absence of asynergy. Am J Cardiol 1976;37:7-11.

14. Van de Ree JK, Zimmerman ANE, Van Heijst ANP: Intoxication by tricyclic antidepressant drugs. Neth J Med 1977;20:149-156.

I5. Biggs JT, Spiker DG, Petit JM, Zlegler VE: Tricyclic antidepressant overdose: Incidence of symptoms. JAMA 1977;238:135-141.

16. Thörstrand C: Clinical features in poisoning by tricyclic antidepressants with special reference to the ECG. Acta Med Scand 1976; 199:377-385.

17. Pellinen TJ, Färkkilä M. Keikkilä J, Luomanmäkj K: Electrocardiographic and clinical features of tricyclic antidepressant intoxication. Ann Clin Res 1987; 19:12-17.

18. Lucas CM, Havenith MG, Van der Veen FH: Changes in canine latissimus dorsi muscle during 24 weeks of continuous electrical stimulation. J Appl Physiol $1992 ; 72: 828-835$.

19. Marshall JD. Focker AD: Cardiovascular effects of tricyclic antidepressant drugs: Therapeutic usage, overdose and management of complications. Am Heart $\mathrm{J}$ 1982; 103:401-414. 
78

20. Kittleson MD, Knowlen GG, Johnson LE: Early and late global and regional left ventricular function after experimental transmural myocardial infarction: Relationships of regional wall motion, wall thickening and global performance. Am Heart J 1987; 1 14:70-78.

21. Smiseth OA, Lindal S, Mjös OD, Vik-mo H. Jörgensen L: Progression of myocardial damage following coronary microembolization in dogs. Acta path microbiol immunol Scand 1983;91:115-124.

22. Hood WD, McCarthy B, Lown B: Aortic pressure loading in dogs with myocardial infarction. Am Heart $J$ 1969:77:55-62.

23. Ellis P'R, Boulis NJ, Viskos JD, Wong SH, Hyland JW: Experimental heart failure in dogs. Arch Surg 1964:89:299-306.

24. Smiseth OA, Mjös OD: A reproducible and stable model of acute ischaemic left ventricular failure in dogs. Clin Phys 1982:2:225-239.

25. Gibbons EF. Hogan RD. Franklin TD, Nolting M, Weyman AE: The natural history of regional dysfunction in a canine preparation of chronic infarction. Circulation 1985:71:394-402.

26. Mettler FP, Young DM. Ward JM: Adriamycine-induced cardiotoxicity in rats. Cancer Res 1977;37:2705-2711.

27. Unverferth DV, Croskery RW, Leier CV, Altshud R, Pipers FS, Thomas J: Canine cobalt cardiomyopathy: A model for the study of heart failure. Am $J$ Vet Res 1983:44:989-995.

28. Van de Ree JK: Cardiovascular effects of intoxication with tricyclic antidepressant drugs; an experimental study. Thesis Utrecht 1971.

29. Sangster B, De Groot G, Borst C, De Wildt D: Dopamine and isoproterenol in Imipramine intoxication in the dog. Clin Toxicol 1985;23:407-420.

30. Delpon E. Valenzuela C. Tamargo J: Tonic and frequency-dependent Vmax block induced by imipramine in guinea pig ventricular muscle fibers. J Cardiovasc Pharmacol 1990; 15:414-420.

31. G Isenberg, $J$ Tamargo: Efiect of imipramine on calcium and potassium currents in isolated bovine ventricular myocytes. Eur J Pharmacol 1985: 108:121-131.

32. Wilkerson RD: Antiarrhythmic effects of tricyclic antidepressant drugs in ouabain-induced arrhythmias in the dog. J Pharmacol Exp Ther 1978;205:666-674.

33. Parmley WW: Mechanics of ventricular muscle. In: Levine HJ, Gaasch WH, eds. The ventricle: Basic and clinical aspects. The Hague, Martinus Nijhoff publishing 1985;41-63.

34. Nattel S, Mittleman M: Treatment of ventricular tachyarrhythmias resulting from amitriptyline toxicity in dogs. J Pharmacol Exp Ther 1984;231:431-435.

35. Shibata S, Norton TR, Izumi T, Matsuo T, Katsuki S: A polypeptide (AP-A) from sea anemone (anthropleura xanthogrammica) with potent positive inotropic action. J Pharmacol Exp Ther 1976:199:298-309.

36. Nattel S. Keable H. Sasyniuk BI: Experimental amitriptyline intoxication: Electrophysiologic manifestations and management. J Cardiovasc Pharmacol 1984: 6:83-89.

37. Fedelesora M, Sulakhe PV, Yates JC, Dhalla NS: Biochemical basis of heart function. Energy metabolism and calcium transport in hearts of vitamin $E$ deficient rats. Can J Physiol thannacol 1971:49:909-918. 
hapter 5

Hemodynamic study following cardiomyoplasty:

\section{Comparison of different stimulation protocols}

Caroline MHB Lucas

Roberto C Lorusso

Marie-Louise Dubelaar

Frederik $\mathrm{H}$ van der Veen

Marc A Vos

Jo Habets

Theo Van der Nagel

Olaf C Penn

Hein JJ Wellens

Submitted for publication 


\title{
Hemodynamic study following cardiomyoplasty:
}

\section{Comparison of different stimulation protocols}

\begin{abstract}
Augmentation of cardiac function has been demonstrated by using the left latissimus dorsi (LD) muscle wrapped around the heart. We studied the effect of different modes of skeletal muscle stimulation both before and after depression of myocardial contractility with imipramine using a versatile pacing device.

Methods: In acute experiments the left LD muscle was dissected distally and wrapped counterclockwisely around the heart in 12 goats. This muscle was stimulated subsequently using an increasing number of pulses ( 1 to 6) with an interpulse interval of $30 \mathrm{msec}$. Pacing was started 20 or 90 msec after the $R$ wave of the $\mathrm{gRS}$.

Results: Summation of outcome of all pacing protocols showed significant increases in left ventricular pressure (LVP), aortic pressure (AoP), left ventricular $\mathrm{dP} / \mathrm{dt}$ ( $\mathrm{LVdP} / \mathrm{dt}$ ), stroke volume of the left ventricle, right ventricular pressure (RVP) and pressure measured in between the left ventricle and the contracting LD muscle (PLD). No significant differences were seen using a delay of $20 \mathrm{msec}$ or $90 \mathrm{msec}$, except for the $\mathrm{LVdP} / \mathrm{dt}$, which did not improve using the longer delay. Infusion of imipramine negatively affected all measured parameters of cardiac contractility. LVP, AoP, RVP and LVdP/dt improved significantly better with a contracting LD muscle after depression of cardiac function compared to the non-failing state, in spite of a significantly less pronounced increase in intermuscular pressure. Before and after imipramine, no effect on left ventricular end-diastolic pressure (LVEdP) was seen demonstrating that ventricular filling was not hampered.

Stimulation of the wrapped LD muscle using an increasing number of pulses progressively improved cardiac function, indicating the need to
\end{abstract}


pace the skeletal muscle with a train of pulses. Conclusion: Contraction of the left latissimus dorsi wrapped around the myocardium improves cardiac output in the acute setting, both before and after depression of cardiac function. While the importance of stimulation of the LD muscle with a train of pulses was demonstrated, no differences were found when using different delays between cardiac and LD muscle contraction.

\section{INTRODUCTION}

For a number of decades, there has been increasing interest in the use of skeletal muscle to assist the failing heart. The first report, using the major pectoral muscle, was published in 1933 by Leriche (1). Termet (2) was the first investigator who described the use of the latissimus dorsi muscle (LD) in 1966. The increasing number of patients suffering from severe cardiac failure with their poor prognosis (3) has further stimulated the use of skeletal muscle wrapped around the heart, as evidenced by a number of recent symposia (4-7).

Essential for the application of skeletal muscle for cardiac assist is transformation of this muscle into a fatigue-resistant muscle by prolonged electrical stimulation. After that period the tissue metabolism of this transformed muscle resembles that of cardiac muscle (8-15).

By using a pulse-train muscle stimulator synchronous to cardiac contraction, a sustained skeletal muscle contraction can be obtained supporting myocardial contraction (16-22).

The aim of this study was to measure the acute hemodynamic effect of contraction of the LD muscle following wrapping around the heart. This was tested both in the normal heart and in an acute failing heart model. Different pacing modes of the LD muscle were evaluated. In particular the effect of skeletal muscle pacing using an increasing number of pulses leading to a tetanic contraction, because of summation of the individual twitches, was studied. We also examined the effect of skeletal muscle stimulation using two different time delays to the depolarization wave of the ventricle.

\section{MATERIALS AND METHODS}

\section{Animal preparation}

Twelve female goats were used with body weights ranging from 28 to $55 \mathrm{~kg}$ $(41 \pm 9 \mathrm{~kg}$ ). All experiments were performed according to the 'Guide for the Care and Use of Laboratory Animals' (23).

Anesthesia was induced by sodium thiopental (Nesdonal) with $15 \mathrm{mg}$ per $\mathrm{kg}$ body weight iv. After endotracheal intubation, anesthesia was maintained with oxygen/ nitrous oxide $(1: 2)$ and $1.5 \%$ Fluothane. During the experiments, the lungs were ventilated with a positive pressure respirator (Pulmomat) and body temperature was maintained constant with a heating blanket. Arterial blood gasses were measured routinely using a blood gas 


\section{2}

analyzer (Radiometer ABL3). Arterial blood oxygen saturation was kept at at least $158 \mathrm{~mm} \mathrm{Hg}$ with supplemental oxygen. Monitoring of arterial $\mathrm{pH}$ and Standard Base excess was performed and if necessary corrected with $4.2 \%$ bicarbonate to maintain the $\mathrm{pH}$ in between 7.35 and 7.45. An indwelling catheter was placed in the right brachial vein for continuous infusion of $5 \%$ Ringer's lactate during the preparation procedure and the experimental protocol. ECG leads I, II and III were recorded simultaneously. Aortic blood pressure (AoP) was measured with a Millar catheter-tip micromanometer (7F Millar PC470), positioned into the aortic arch through the femoral artery. Left ventricular pressure (LVP) measurements were obtained with a Millar catheter-tip micromanometer (7F Millar PC470), inserted through the left brachial artery. Left ventricular end-diastolic pressure (LVEdP) was separately recorded by clipping the left ventricular pressure at $20 \mathrm{mmHg}$ and subsequently amplifying the signal on the recorder. The first derivative (positive and negative LVdP/dtmax) was determined as described by Schaper et al (24). Right ventricular pressure (RVP) was measured using a Millar catheter-tip micromanometer, inserted into the right ventricle via the left jugular vein. All catheters were flushed repeatedly with saline.

\section{Pedicle preparation}

A leftsided midaxillary incision was performed and all collateral blood vessels to the distal part of the LD muscle were coagulated. All attachments of the muscle were disconnected except the axillary pedicle to keep the thoracodorsal artery, vein and nerve intact. Two intramuscular electrodes (Medtronic SP5528) were implanted in the upper part of the LD muscle flap, perpendicular to the main branches of the thoracodorsal nerve, as described by Chachques and coworkers (25).

Threshold value of stimulation was measured during temporary connection of these electrodes to a stimulator (Medtronic SP3028).

\section{Wrapping procedure}

A partial resection of the anterior arch of the second rib was performed to transfer the muscle and pacing leads into the thorax. The thoracic cavity was opened at the fifth left intercostal space and the pericardium removed. A sensing electrode (Medtronic SP5548) was implanted in the left ventricular wall. Subsequently, the left LD muscle was wrapped in a counterclockwise fashion around both ventricles. The muscle was first positioned around the right ventricle and fixed near the atrioventricular groove at the base of the heart with interrupted sutures, followed by wrapping the remaining part of the muscle around the left ventricle. This distal portion was sutured to the proximal part of the muscle, as described by Anderson et al (26). In the animals used, the skeletal muscle surface was larger than the surface of both ventricles and therefore, the most distal part of the LD muscle was sutured on top of the first layer above the anteroseptal wall of both ventricles.

During the wrapping procedure, a flat Silastic balloon $(2 \times 2 \mathrm{~cm})$ as used by Smiseth and coworkers $(27,28)$, was fixed with sutures between the apex of 
the left ventricle and the left LD muscle. The balloon was filled with $3 \mathrm{ml}$ of saline and connected to an Ailtech pressure transducer. Calibration of the balloon was performed by inserting the balloon in a closed bottle filled with air. The pressure inside the bottle was measured with an Ailtech pressure transducer and equilibration of both pressures was performed by changing the pressure inside the bottle. The balloon was calibrated from $\mathrm{O} \mathrm{mm} \mathrm{Hg}$ to maximally $100 \mathrm{mmHg}$. This balloon was used to measure the pressure in between the heart and the wrapped LD muscle (PLD).

An electromagnetic flow probe (Skalar) was connected to a sine wave electromagnetic flowmeter (Transflow 600) and positioned around the ascending aorta to measure aortic flow. (All pressure transducers were calibrated prior to each set of measurements).

Variables were recorded continuously on a multichannel Schwarzer recorder at a speed of $1.25 \mathrm{~mm} / \mathrm{sec}$. and, intermittently, at a speed of 25 $\mathrm{mm} / \mathrm{sec}$ during electrical stimulation of the LD muscle.

\section{Experimental protocol}

The goats recovered from the wrapping procedure during at least half an hour before the LD muscle was stimulated. Three goats appeared to be unsuitable for further evaluation because of atrial fibrillation ( 2 goats) or inability to stimulate the wrapped muscle (one goat). In the latter goat the LD muscle appeared to be severely ischemic, due to accidental interruption of the arterial blood supply.

In the remaining 9 goats the intramuscular electrodes and the sensing electrode were connected to a pacing device (Prometheus, Medtronic) to stimulate the muscle. This system allows selection of pacing parameters like synchronization delay, strength, burst duration, interstimulus interval(s) and number of stimuli, and also has Holter capabilities (29). Stimulation of the wrapped LD muscle was performed using pulses, having a pulse width of $180 \mu$ seconds and an amplitude of 5 volt. The number of pulses was sequentially increased from 1 to 6 . The interpulse interval was programmed at $30 \mathrm{msec}$. During the longest pulse train $(n=6)$. effect of both interpulse intervals of 20 and $30 \mathrm{msec}$ were studied. All pace protocols used a delay from the sensed R-wave to the first stimulus of $20 \mathrm{msec}$ or $90 \mathrm{msec}$. Table 1 shows the content and sequence of the stimulation protocol. The synchronization ratio was programmed in an 1:8 mode (muscle to heart contraction). This low synchronization rate was selected to avoid the occurrence of fatigue of the unconditioned LD muscle. During each stimulation protocol, the wrapped LD muscle was stimulated 5 times. In between the different protocols there was a pause of 1 minute.

After completion of the whole pacing protocol, depression of cardiac contractility was created with continuous infusion of imipramine (Tofranil ${ }^{R}$, CibaGeigy, Basel, Switserland) at a dosage of $7.5 \mathrm{mg} / \mathrm{kg}$ body weight $[30,31$ ). Following 30 minutes of imipramine infusion, the LD muscle was again paced according to the protocol, shown in Table 1. Again, a total of 50 contractions were recorded. 
84

TABLE 1.

Successive stages of the stimulation protocol

\begin{tabular}{llll}
\hline protocol & $\begin{array}{l}\text { number of pulses in } \\
\text { a burst }\end{array}$ & $\begin{array}{l}\text { interpulse interval } \\
\text { (msec) }\end{array}$ & $\begin{array}{l}\text { sensing delay } \\
\text { (msec) }\end{array}$ \\
\hline A & 1 & & 20 \\
A1 & 1 & & 90 \\
B & 2 & 30 & 20 \\
B1 & 2 & 30 & 90 \\
C & 3 & 30 & 20 \\
C1 & 3 & 30 & 90 \\
D & 6 & 30 & 20 \\
D1 & 6 & 30 & 90 \\
E & 6 & 20 & 20 \\
E1 & 6 & 20 & 90 \\
\hline
\end{tabular}

At the end of the experiment, the goats were sacrificed with an overdose of sodium pentobarbitone.

\section{Data analysis}

During each stage of the pacing protocol, 10 non-assisted and all 5 assisted beats were measured during continuous respiration, because respiratory arrest lead to both an immediate rise in right ventricular pressure and the induction of extra beats. The control beats were measured immediately before the first assisted beat, equally divided between inspiration and expiration. The 10 non-stimulated and 5 stimulated beats during every stage of the stimulation protocol were averaged per protocol for each goat. For each parameter, the average of these 10 protocols in 9 goats $(n=90)$ was determined.

Stroke volume of the left ventricle (SV) was measured, using the aortic flow curve. The surface area under the curve was determined, using a digitizer [MOP videoplan Kontron] with a cross hair cursor. Calibration was performed by measuring the area of 20 aortic flow curves, obtained before the start of LD muscle stimulation, relative to the integrated aortic flow per minute. Measurements, made during the execution of the pacing protocol were related to the mean value of these 20 beats.

\section{Statistical analysis}

One way analysis of variance was used to compare the non-assisted beats during all pacing protocols for 9 goats, both before and after induction of a failing heart. Assisted and non-assisted beats were compared, using Student's t test for unpaired events. One way analysis of variance followed by Student's t test for unpaired events was also used to evaluate the effect of the different pacing protocols. Linear regression and correlation coefficient were calculated, to compare the absolute increase in intermuscular pres- 
TABLE 2.

Hemodynamic results of assisted versus unassisted beats ( $X \pm S E M, n=90$ )

\begin{tabular}{llllllll}
\hline & $\begin{array}{l}\text { SV } \\
(\mathrm{ml})\end{array}$ & $\begin{array}{l}\text { RVP } \\
(\mathrm{mmHg})\end{array}$ & $\begin{array}{l}\text { PLD } \\
(\mathrm{mmHg})\end{array}$ & $\begin{array}{l}\text { LVP } \\
(\mathrm{mmHg})\end{array}$ & $\begin{array}{l}\text { AoP } \\
(\mathrm{mmHg})\end{array}$ & $\begin{array}{l}\text { LVdP/dt } \\
(\mathrm{mmHg} / \mathrm{sec})\end{array}$ & $\begin{array}{l}\text { LVEdP } \\
(\mathrm{mmHg})\end{array}$ \\
\hline $\mathrm{C}$ & $14.4 \pm 0.5$ & $25.5 \pm 0.5$ & $13.7 \pm 0.6$ & $78.0 \pm 2.3$ & $79.0 \pm 2.4$ & $1115 \pm 38$ & $9.6 \pm 0.4$ \\
$\mathrm{~S}$ & $18.4 \pm 0.6$ & $39.0 \pm 1.2$ & $37.6 \pm 2.1$ & $90.0 \pm 2.7$ & $88.0 \pm 2.8$ & $1240 \pm 42$ & $9.4 \pm 0.4$ \\
$\mathrm{p}$ & $<0.001$ & $<0.001$ & $<0.001$ & $<0.001$ & 0.015 & 0.03 & N.S. \\
\hline
\end{tabular}

Abbreviations: AoP $=$ aortic pressure, $\mathrm{C}=$ unassisted beats, LVEdP $=$ left ventricular end-diastolic pressure, $\mathrm{LVdP} / \mathrm{dt}=$ pos. $\mathrm{dP} / \mathrm{dt}$ of the left ventricle, LVP $=$ left ventricular pressure. $\mathrm{PLD}=$ intermuscular pressure. $\mathrm{RVP}=$ right ventricular pressure, $\mathrm{S}=$ assisted beats. $\mathrm{SV}=$ stroke volume of the left ventricle.

sure during stimulation with AoP, LVdP/dt, RVP. and SV of the left ventricle. Differences were accepted to be statistically significant at $p<0.05$. Values are presented as mean \pm 1 standard error of the mean.

\section{RESULTS}

Before induction of cardiac failure

During all 10 pacing protocols, no significant changes in control hemodynamic values were seen. Therefore, it was considered legitimate to assess the mean of the non-assisted beats of all 10 pacing protocols in 9 goats ( $\mathrm{n}$ $=90$ ) as the control value.

Stimulation of the LD muscle had a significant positive effect on right- and leftsided heart function (RVP, LVP, LVdP/dt, AoP, SV of the left ventricle) and intermuscular pressure (PLD). This is summarized in Table 2 (A, A1, B, B 1, C, C1, D, D1, E, E 1: n = 90). Especially, the effect on RVP appeared very pronounced. Positive nor negative effects were seen on LVEdP.

In figure 1. the pooled results of pacing with the short $20 \mathrm{msec}$ (stimulation protocols $\mathrm{A}, \mathrm{B}, \mathrm{C}, \mathrm{D}, \mathrm{E} ; \mathrm{n}=45$ ) versus the long $90 \mathrm{msec}$ (stimulation protocols Al, B l, Cl, D 1, E 1; n = 45) sensing delay are shown. A consistent significant improvement was observed for all hemodynamic parameters, irrespective of the stimulation delay, except for the LVdP/dt. With a delay of $90 \mathrm{msec}$, there was no effect of latissimus dorsi stimulation on this parameter.

The individual effects of pacing protocols (Table 1) with an increasing number of pulses (A. B, C, D) and a burst with a short interpulse interval (E; $20 \mathrm{msec}$ ) are shown in figure 2 . As anticipated, a train of pulses resulted in an increased assistance of cardiac function. Stroke volume of the left ventricle, RVP, LVP and AoP demonstrated a significant increase using 6 pulses as compared to 1 pulse stimulation (protocol A) with a $30 \mathrm{msec}$ and a $20 \mathrm{msec}$ interpulse interval (protocol $D+E$ ). On using single pulse stimulation (protocol A), a pronounced increase of $14 \pm 2.5 \%$ in $\mathrm{LVdP} / \mathrm{dt}$ was observed. Although a tendency to improvement was seen using more pulses, 
86

LVP

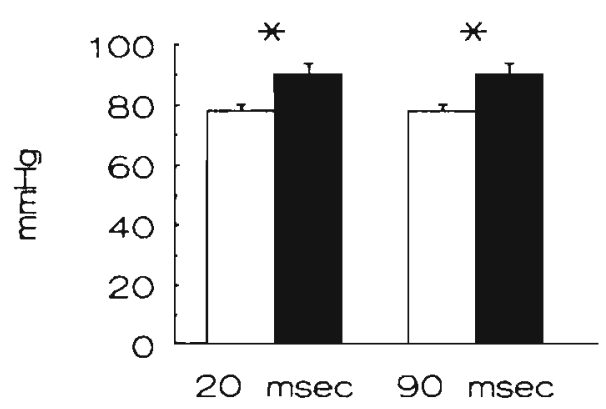

$\mathrm{AOP}$

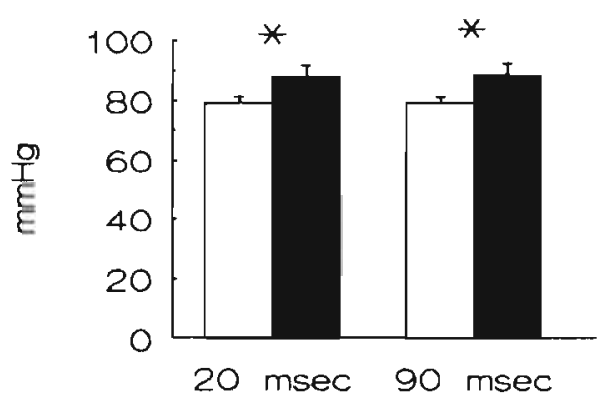

$L \vee d P / d t$

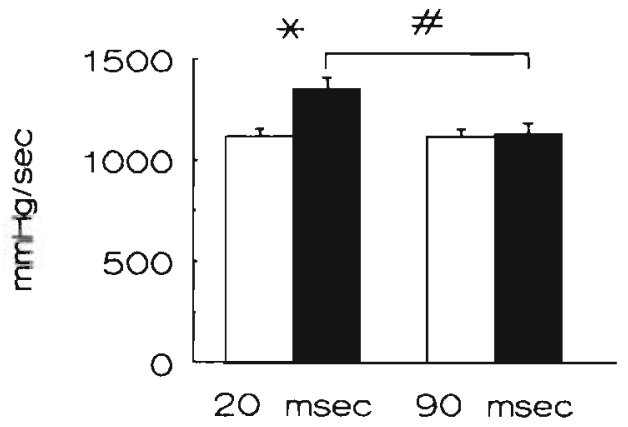

SV

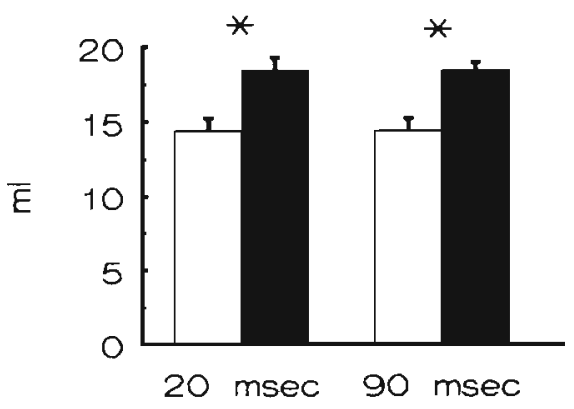

RVP

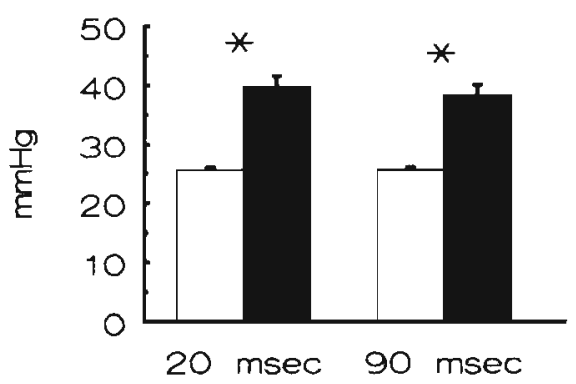

PLD

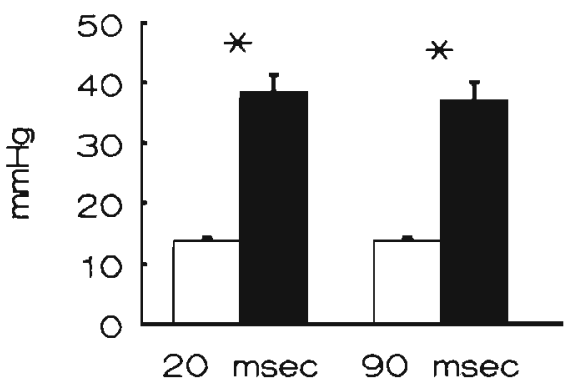

\section{FIGURE 1.}

The effect of pacing the $L D$ muscle with a short $(20 \mathrm{msec}$ ) and a long delay (90 msec) in the absence of cardiac failure. AoP $=$ aortic pressure, $L V d P / d t=$ positive left ventricular $d P / d t . L V P$ $=$ left ventricular pressure. $P L D=$ intermuscular pressure. $R V P=$ right ventricular pressure, $S V$ = stroke volume of the left ventricle. White bars represent non-assisted beats, dark bars represent assisted beats. Values are presented as mean \pm SEM $(n=45) . *$ significant difference between non-assisted and assisted beats $(p<0.05)$. \#: significant difference between assisted beats using a short or a long delay $(p<0.05)$. 

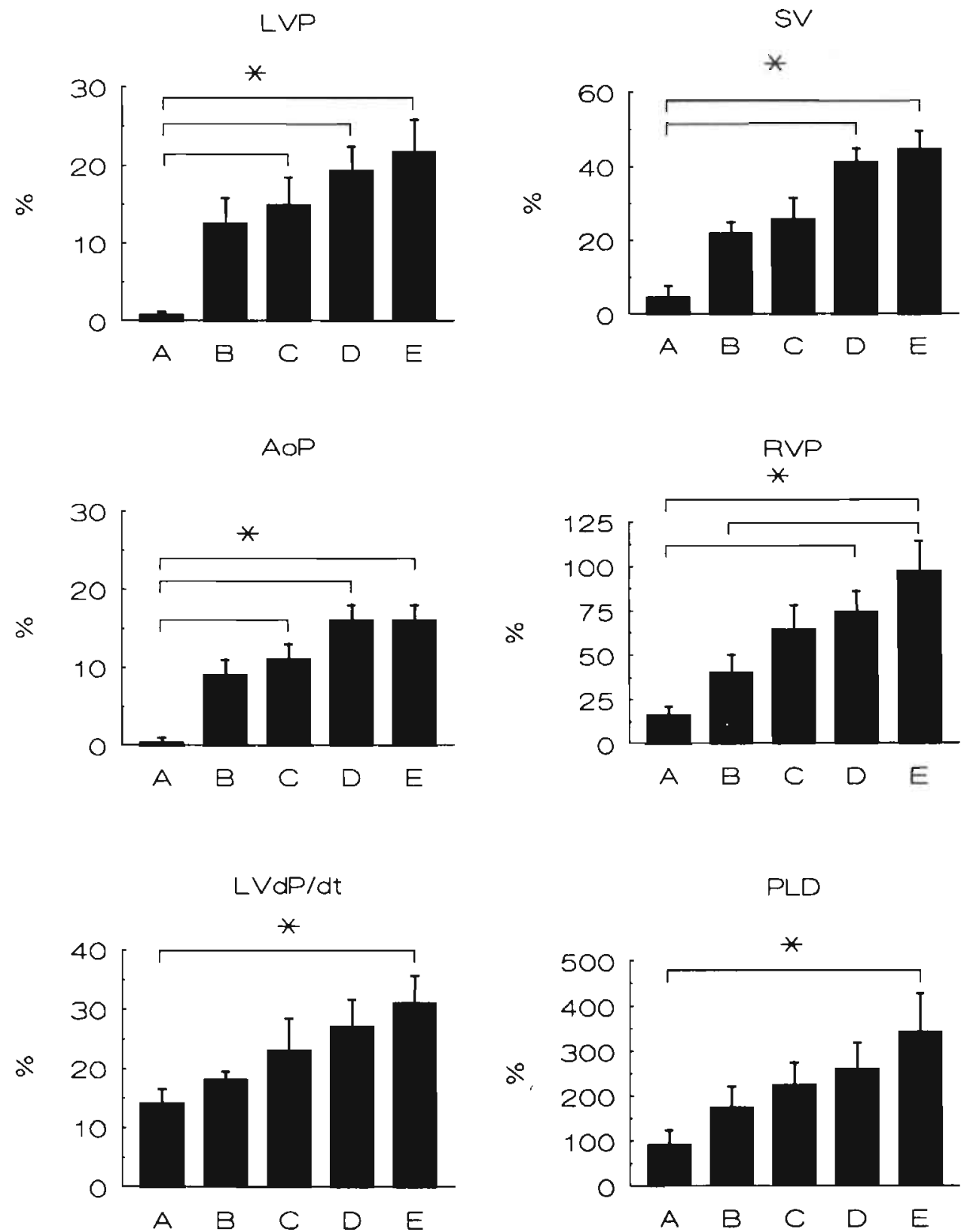

FIGURE 2.

Percentage of increase in aortic pressure (AoP), left ventricular dP/dt (LVdP/dt), left ventricular pressure (LVP), intermuscular pressure (PLD), right ventricular pressure (RVP) and stroke volume. (SV) using the different stimulation protocols A, B, C, D and E as compared to unassisted beats. Values are presented as mean \pm SEM $(n=45)$. *: significant difference between two stimulation protocols $(p<0.05)$. 
TABLE 3.

Relation between the absolute increase in PLD with SV. RVP. LVP. AOP and LVdP/dt both before and during cardicac depression with imipramine.

\begin{tabular}{|c|c|c|c|c|c|}
\hline & $\begin{array}{l}\text { SV } \\
\text { (ml) }\end{array}$ & $\begin{array}{l}\text { RVP } \\
\text { (mmHg) }\end{array}$ & $\begin{array}{l}\text { LVP } \\
\text { (mmHg) }\end{array}$ & $\begin{array}{l}\text { AoP } \\
(\mathrm{mmHg})\end{array}$ & $\begin{array}{l}\mathrm{LVdP} / \mathrm{dt} \\
(\mathrm{mmHg} / \mathrm{sec})\end{array}$ \\
\hline \multicolumn{6}{|c|}{ Before imipramine $(n=90)$; } \\
\hline r & 0.479 & 0.740 & 0.793 & 0.710 & 0.750 \\
\hline $\mathrm{p}$ & $<0.001$ & $<0.001$ & $<0.001$ & $<0.001$ & $<0.001$ \\
\hline \multicolumn{6}{|c|}{ After imipramine ( $n=60$ ): } \\
\hline r & 0.729 & 0.882 & 0.846 & 0.844 & 0.697 \\
\hline $\mathrm{p}$ & $<0.001$ & $<0.001$ & $<0.001$ & $<0.001$ & $<0.001$ \\
\hline
\end{tabular}

Abbreviations: See legends to Table 2. For LVdP/dt only results of measurements with the short delay to the R-wave are used.

a significant change was observed using a burst of 6 pulses with an interpulse interval of $20 \mathrm{msec}$ (protocol E). Single pulse stimulation resulted in an increase in PLD of $90 \pm 34 \%$. Due to the large variation in increase in intermuscular pressure, only protocol $E$ resulted in a significant difference as compared to protocol $A$. The relative increase in AoP and LVP, however, was already significantly higher than 1 pulse stimulation, when only 3 pulses (protocol C) were applied. RVP also demonstrated differences between 2 pulses (protocol B) and 6 pulses (protocols D and E) stimulation. No significant difference was obtained between a burst of 6 pulses with either an interpulse interval of $20 \mathrm{msec}$ (protocol E) or of $30 \mathrm{msec}$ (protocol D). Correlation between the absolute increase in PLD during LD muscle stimulation and the hemodynamic variables was measured, to obtain information about the role of increased intermuscular pressure to improve cardiac function. Data are shown in Table 3, using a compilation of the results of all protocols (A-E). Obviously a clear correlation was present will all variables.

\section{After cardiac failure}

The results from 6 of the 9 goats could be used for evaluation of LD muscle contraction following the infusion of imipramine. Three animals developed intractable atrial fibrillation, because of mechanical irritation of the right atrial wall by the right ventricular catheter.

Figure 3 demonstrates both the influence of imipramine on the measured parameters and the effect of pacing of the wrapped LD muscle on hemodymamic function. Again, results of all pacing protocols of 6 goats are compiled. Following 30 minutes of imipramine infusion, significant decreases in all hemodynamic values were seen, except for the intermuscular pressure. There were no differences in values between successive beats. indicating stability of the failing heart model. Concomitantly, cardiac frequency decreased from $119 \pm 6$ to $98 \pm 7$ beats $/ \mathrm{min}$ ( $p=0.04$ ). LVEdP 
showed a small increase from $9.6 \pm 0.4$ to $11.9 \pm 0.3(\mathrm{p}<0.001)$. As demonstrated, also after depression of cardiac contractility, significant improvements in LVP, AoP, LVdP/dt, RVP and SV of the left ventricle were obtained, when LD pacing was performed. Again no influence was seen on the LVEdP (11.9 \pm 3.1 with stimulation).

The difference in percentage hemodynamic support of the contracting LD muscle, before and after depression of cardiac contractility is demonstrated in figure 4. This shows a significant increase in relative improvement of RVP, LVP. AoP and LVdP/dt after imipramine infusion. These increases were obtained, despite the fact that the percentage in increase in intermuscular pressure was significantly less after the administration of imipramine (120 $\pm 15 \%$ compared to $154 \pm 18 \%$ before imipramine). No significant difference in percentage in increase in SV of the left ventricle was seen.

Correlation between the absolute increases in PLD with AoP. RVP and SV appeared to be better after the induction of cardiac failure as demonstrated in Table 3. 


\section{0}
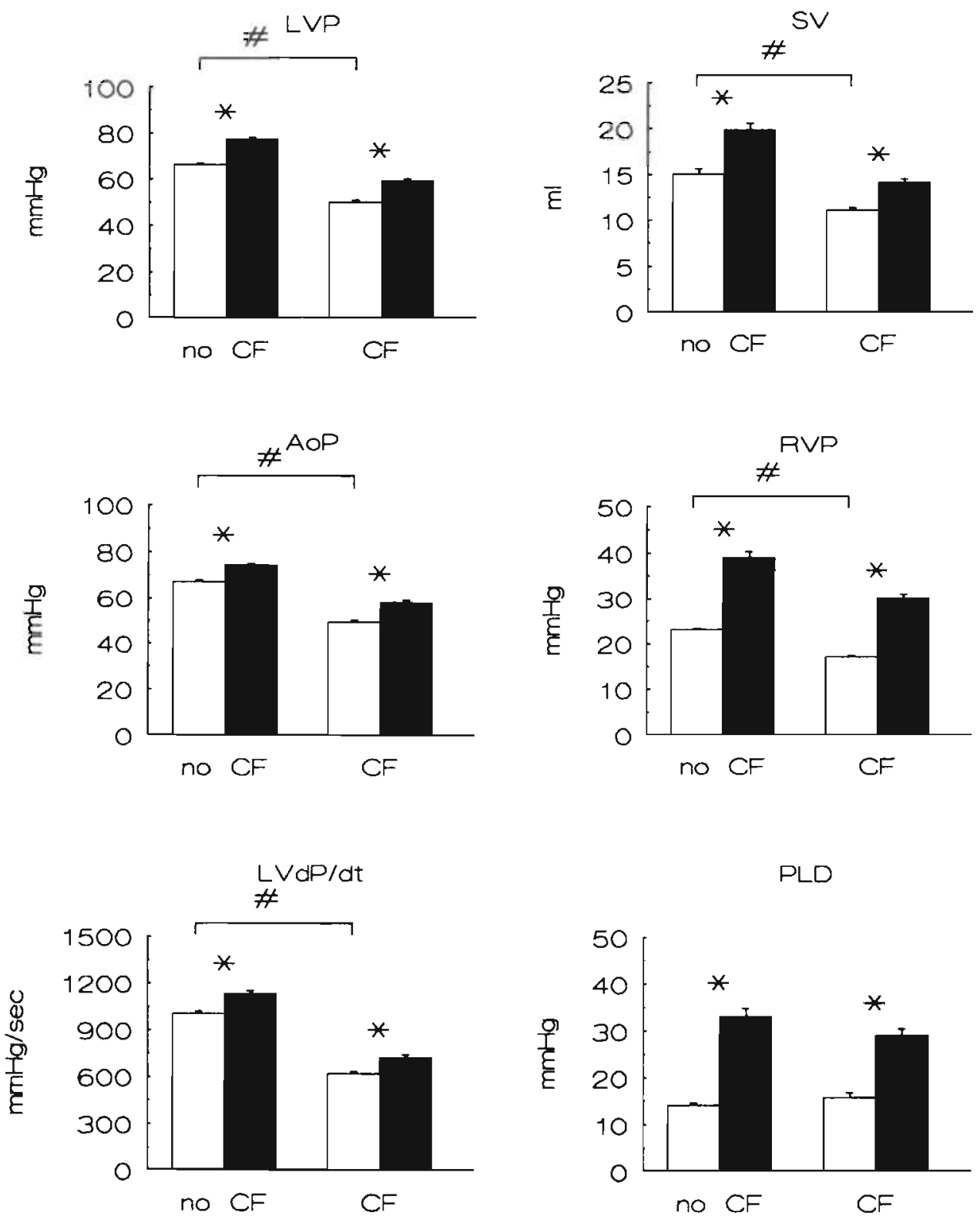

FIGURE 3.

Effect of stimulating the $L D$ muscle before (no $C F$ ) and during cardiac failure (CF). Values are presented as mean \pm SEM $(n=60$ before and after cardiac failure). For the labeling of hemodynamic variables. see legends to figure 1. ": significant difference between assisted and unassisted beats $(p<0.05)$. \#: significant difference between the unassisted beats without (no $C F)$ and with cardiac failure (CF) $(p<0.05)$. 

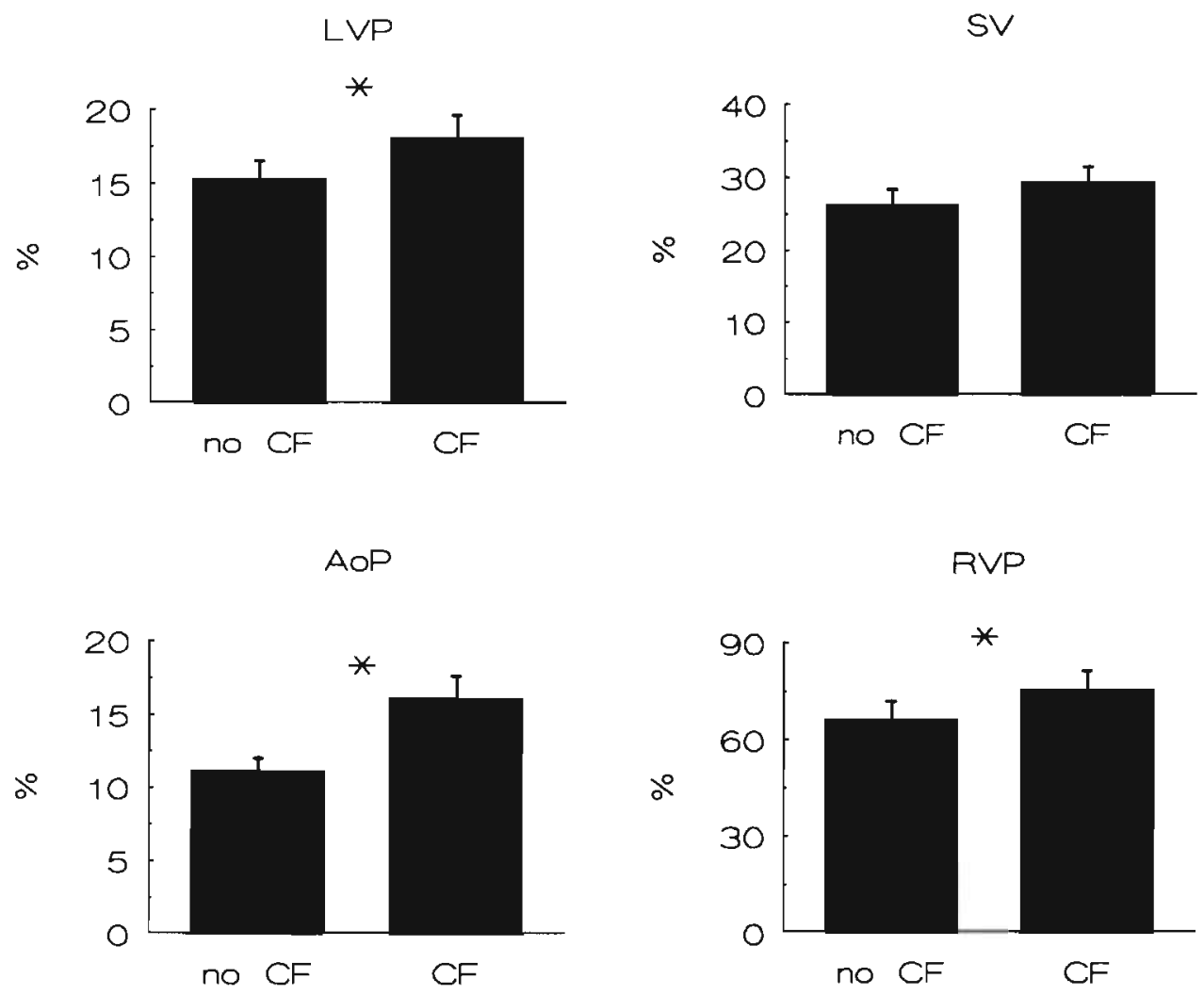

$L V d P / d t$
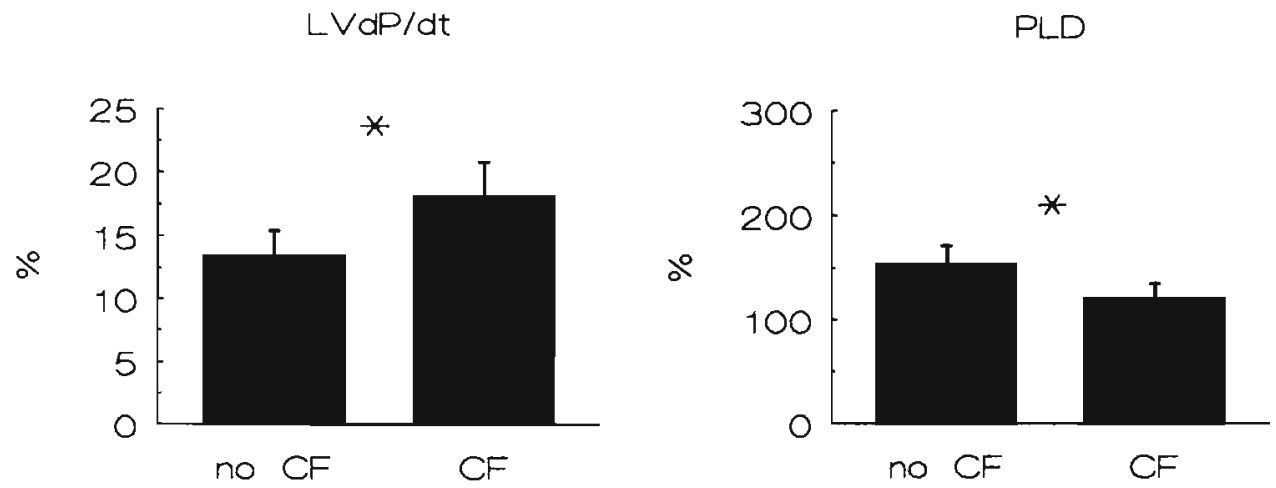

FIGURE 4.

Changes in AoP, LVdP/dt, LVP. PLD. RVP and SV in percentage during assisted beats relatwe to unassisted beats, before (no $C F$ ) and during cardiac failure (CF). Values are presented as mean $\pm S E M$ ( $n=60$ before and after cardiac failure). See legends to figure 3 . *: significant difference in percantage in of improvement without and with cardiac fallure $(p<0.05)$. 


\section{DISCUSSION}

The present study shows that contraction of the LD muscle, wrapped around both ventricles, can improve cardiac function in the acute setting, and can partly abolish the negative inotropic effect of imipramine on cardiac contractility. Adherence of the wrapped LD muscle to the myocardium was evaluated, using an intermuscular balloon, which demonstrated in all goats a 'pressure wave', due to recurrent filling and subsequent emptying of the heart. Tracings from this balloon also demonstrated the increase in intermuscular pressure during contraction of the LD muscle (figure 5).

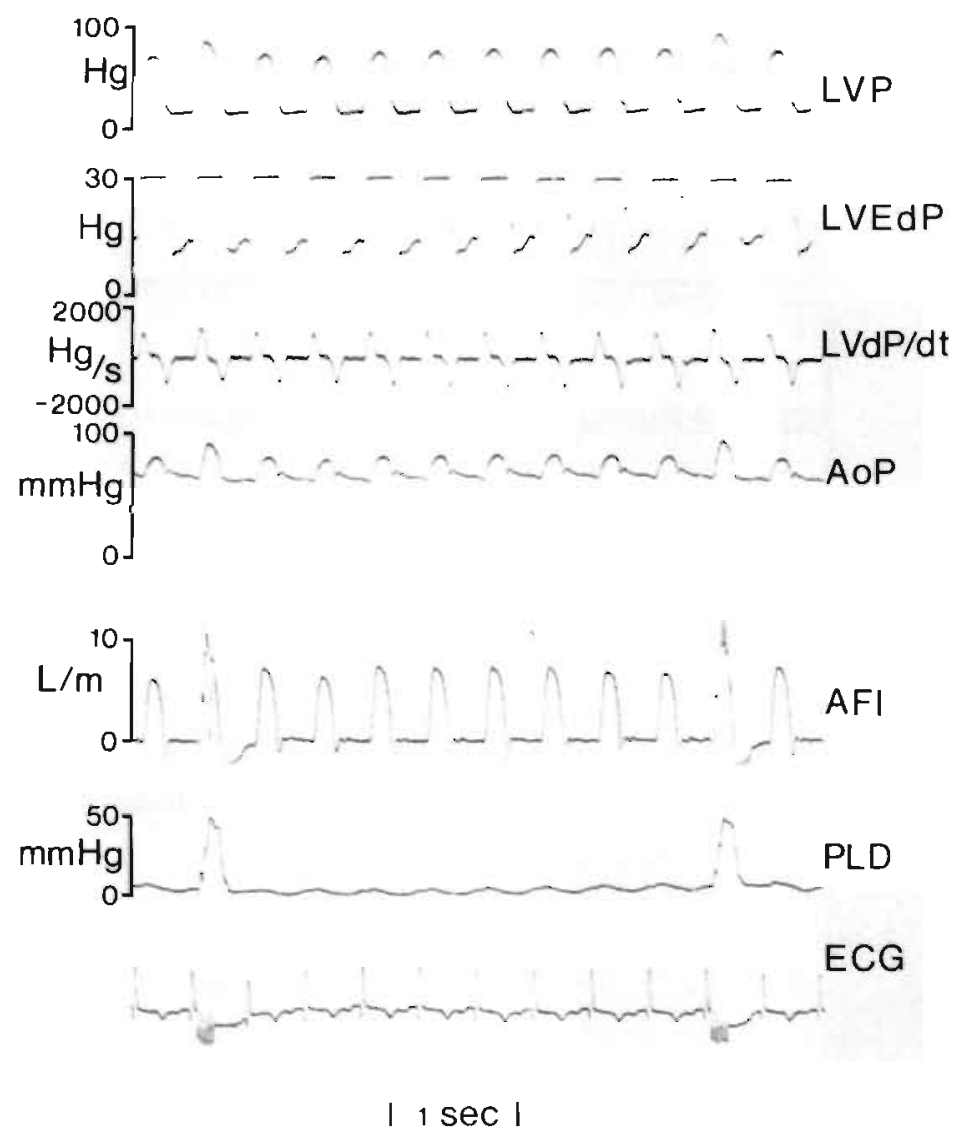

\section{FIGURE 5.}

Registration showing the effect of synchronous burst pacing of the wrapped LD on different parameters of cardiac function. Abbreviations: $A \circ P=$ Aortic pressure, $A O F=$ Aortic flow, $d P / d t=$ positive $d P / d t$ of the left ventricle, $L V E d P=$ left ventricular end-diastolic pressure. $L V P=l e f t$ ventricular pressure. $L / m=$ litres per min. $P L D=$ pressure in between the left ventricle and the wrapped LD muscle. 
No obstruction of ventricular filling was observed, since the left ventricular end-diastolic pressure did not change, due to the wrapped and contracting LD muscle.

Our findings show that in particular the right ventricle can benefit from the assistance of a wrapped skeletal muscle, which is in accordance with the literature $(32,33)$. This is not surprising, because right ventricular pressure is significantly lower than left ventricular pressure during the ejection phase.

Although the optimal sensing delay from the R-wave to the pacing stimulus of the wrapped muscle is still a matter of debate $(34,35,36)$, a sensing delay of $4 \mathrm{msec}$ is commonly used (25). In our studies, a delay of $20 \mathrm{msec}$ was compared with a delay of $90 \mathrm{msec}$. No significant differences were seen for all parameters, except for the left ventricular dP/dt. Pacing the wrapped LD muscle with a longer delay of $90 \mathrm{msec}$ results in assistance of the myocardium, following the steep part of the left ventricular pressure curve. Therefore, no effect on the left ventricular $\mathrm{dP} / \mathrm{dt}$ is observed, which makes this variable only valid to measure myocardial assist (37) when a short delay is used.

This is the first study in which the differences in assistance of the heart after a dynamic cardiomyoplasty procedure are measured, when using an increasing number of pacing pulses. Although significant improvement in myocardial function already occurs using only l pulse stimulation (34), it appears essential to pace the wrapped muscle with a train of pulses. This will result in a tetanic contraction of the skeletal muscle, due to temporal summation. In the present study, however, LVdP/dt demonstrated a significant improvement with only 1 pulse stimulation, without important additional improvement when more pulses were added. This can be explained by the fact that the first pulse occurs during the isovolumic contraction phase, that is during the steep part of the left ventricular pressure curve, as was mentioned during the discussion on the importance of the sensing delay. In our protocol, a train of maximally 6 pulses was used. resulting in a burst duration of maximally $160 \mathrm{msec}$. It was necessary to select this relatively short burst duration, because the same burst was also tested with a delay of $90 \mathrm{msec}$. Therefore, the final pulse of this burst occurs 250 msec after sensing the R-wave, which is at the end of the systolic ejection period.

No significant differences were observed between the bursts with interpulse intervals of $20 \mathrm{msec}$ and $30 \mathrm{msec}$. A reason for this might be that the burst duration with interpulse intervals of $20 \mathrm{msec}$ totals only $110 \mathrm{msec}$, compared to $160 \mathrm{msec}$ when interpulse intervals of $30 \mathrm{msec}$ are used.

Infusion of imipramine significantly impaired myocardial contractility, as has been described earlier $(30,31)$. In that situation, improvement in cardiac function appeared even more pronounced, considering the percentage of increase in right ventricular pressure, left ventricular pressure, aortic pressure and left ventricular $\mathrm{dP} / \mathrm{dt}$, indicating the suitability of the stimulated wrapped LD muscle to support a failing heart. This was obtained despite the fact that the percentage of increase in intermuscular pressure 
was less, pointing to a reduced contractile force of the $\mathrm{LD}$ muscle. As movement of the balloon was not likely, since adequate fixation was obtained and no negative effect of imipramine on contractile force was seen in experiments with in situ stimulated LD muscle (31), the main reason for a reduced force production could be the negative effect of repeatedly stimulating the unconditioned muscle, immediately after the wrapping procedure (19, 20, 38). Moreover, reduced contractile force of the LD muscle after imipramine could account for the observation that no relative increase in stroke volume of the left ventricle was seen after imipramine, compared to the non-falling state.

The large variation in rise in intermuscular pressure during contraction of the wrapped LD muscle points to the difficulty to obtain uniform wrapping. This is supported by the observation that the correlation between the increase in PLD and measured parameters improved after induction of cardiac failure, due to dilatation of the heart. Another reason could be the torsion of the heart during skeletal muscle contraction which could have resulted in partial obstruction of left ventricular outflow. This change in orientation of the heart, during contraction of the LD muscle in the opened thorax preparation was also reported by Anderson et al (26). (It is probably less important in the closed chest animal preparation).

In conclusion; the left latissimus dorsi muscle wrapped around the heart in a counterclockwise direction, significantly improves cardiac function both before and after the creation of a failing heart. Maximal improvement was obtained by using a train of pulses, resulting in a tetanic contraction of the skeletal muscle. On using a maximal burstduration of $150 \mathrm{msec}$, no differences were observed in the measured pressure and flow parameters. when a short or long sensing delay to the R-wave was programmed, with the exception of the left ventricular $\mathrm{dP} / \mathrm{dt}$. 


\section{REFERENCES}

1. Leriche R.: Essai experimentale de traitment de certains infarctus du myocarde et de l'aneurysme du coeur par une greffe de muscle strié. Biull Soc. Nat Chir 1933:59:229-234.

2. Termet H, Chalencon JL, Estour F: Transplantation sur le myocarde d'un muscle strié excité par pacemaker. Ann Chir Thorac Cardio 1966;5:270-276.

3. Franciosa JA. Wilen M, Ziesche S, Cohn JN: Survival in men with severe chronic left ventricular failure due to either coronary heart disease or idiopathic dilated cardiomyopathy. Am J Cardiol 1983;51:831-836.

4. Biomechanical cardiac assist: Cardiomyoplasty and muscle-powered devices. Chiu RC-J (Ed.). Futura Publishing Company Inc.. Mount Kisco, NY 1986.

5. Transformed muscle for cardiac assist and repair. Chiu RC-J, Bourgeois IM (Eds.). Futura Publishing Company Inc., Mount Kisco, NY 1990.

6. Cardiomyoplasty. Carpentier A, Chachques JC. Grandjean P (Eds.). Futura Publishing Company Inc., Mount Kisco, NY 1991.

7. J Cardiac Surg 1991;6(suppl). Stephenson LW (Ed.). Futura Publishing Company Inc., Mount Kisco, NY.

8. Acker MA. Mannion JD. Brown WE, Salmons S, Henriksson J, Bitto T, Gale DR. Hammond R, Stephenson LW: Canine diaphragm muscle after I yr of continuous electrical stimulation: its potential as a myocardial substitute. J Appl Physiol 1987;62: 1264-1270.

9. Macoviak JA, Stephenson LW, Amenti F. Kelly AM. Alavi A. Mackler T, Cox J, Palatianos G, Edmunds LH: Electrical conditioning of in situ skeletal muscle for replacement of myocardium. J Surg Res 1982:32:429-438.

10. Mannion JD. Bitto T. Hammond RL, Rubinstein NA, Stephenson LW: Histochemical and fatigue characteristics of conditioned canine latissimus dorsi muscle. Circ Res 1986:58:298-304.

11. Ianuzzo CD, Hamilton N, O'Brien PJ, Desrosiers C. Chiu R: Biochemical transformation of canine skeletal muscle for use in cardiac assist devices. J Appl Physiol 1990;68:1481-1485.

12. Salmons S, Hendriksson J: The adaptive response of skeletal muscle to increased use. Muscle \& Nerve 1981:4:94-105.

13. Brown MD, Cotter MA. Hudlicka O. Vrbova G: The effects of different patterns of muscle activity on capillary density, mechanical properties and structure of slow and fast rabbit muscles. Pflügers Arch 1976:361:241-250.

14. Pette D: Activity-induced fast to slow transitions in mammalian muscle. Med Sci Sports Exc 1984; 16:517-528.

15. Lucas CM, Havenith MG, Van der Veen FH, Habets J, Van der Nagel T, Schrijvers - van Schendel JM. Penn OC, Wellens HJ. Changes in canine latissimus dorsi muscle during 24 weeks of continuous electrical stimulation. J Appl Physiol 1992: 92;828-835.

16. Drinkwater DC, Chiu RCJ. Modry D: Cardiac assist and myocardial repair with synchronously stimulated skeletal muscle. Surg Forum 1980:31:271-273.

17. Dewar ML. Chiu RCJ: Cardiomyoplasty and the pulse-train stimulator. In Chiu RC-J (Ed.): Biomechanical Cardiac Assist: Cardiomyoplasty and Muscle-Powered Devices. Futura Publishing Company, Inc., Mount Kisco, NY 1986;43-58.

18. Dewar ML. Drinkwater DC. Wittnich C, Chiu RCJ: Synchronously stimulated skeletal muscle graft for myocardial repair. J Thorac Cardiovasc Surg 1984: 87:325-331.

19. Acker MA, Hammond RL, Mannion JD, Salmons S. Stephenson LW: An autologous biologic pump motor. J Thorac Cardiovasc Surg 1986;92:733-746. 
96

20. Mannion. JD, Acker MA, Hammond RL, Faltemeyer W, Duckett S, Stephenson LW: Power output of skeletal muscle ventricles in circulation: short-term studies. Circulation 1987:76:155-162.

21. Kochamba G. Desrosiers C, Dewar M, Chiu RCJ: The muscle-powered dualchamber counterpulsator: Rheologically superior implantable cardiac assist device. Ann Thorac Surg 1988;45:620-625.

22. Anderson WA, Bridges CR, Chin AJ, Andersen JS, Acker MA, Hammond RL, Dimeo F, Calahan PT, Gale DR, Brown WE. Stephenson LW: Long-term neurostimulation of skeletal muscle: Its potential for a tether-free biologic cardiac assist device. PACE 1988;11:2128-2134.

23. National Academy of Sciences: Guide for the Care and Use of Laboratory Animals. DHHS publication No. NIH 85-23, revised 1985.

24. Schaper W, Lewi P. Jageneau AHM: The determination of the rate of change of the left ventricular pressure [dP/dt]. Arch Kreislaufforsch 1965:46:27-41.

25. Chachques JC, Grandjean P, Schwartz K, Mihaileanu S, Fardeau F, Swyngedauw B. Fontaliran F, Romero N, Wisnewsky C. Perier P. Chauvaud S, Bourgeois I. Carpentier A: Effect of latissimus dorsi dynamic cardiomyoplasty on ventricular function. Circulation 1988;78(suppl III):203-216.

26. Anderson WA, Andersen JS. Acker MA, Hammond RL, Chin AJ, Douglas PS, Khalafalla AS, Salmons S. Stephenson LW: Skeletal muscle grafts applied to the heart: A word of caution. Circulation 1988;78(suppliII): 180-190.

27. Smiseth OA, Scott-Douglas NW, Traboulsi M. Stone JA, Smith ER, Tyberg JV: The role of the pericardium: Experimental aspects. Heart Failure 1987;3:6-12.

28. Smiseth OA, Frais MA, Kingma I, Smith ER, Tyberg JV: Assessment of pericardial constraint in dogs. Circulation 1985:71:158-164.

29. Grandjean PA, Bakels N, Berne E, Leinders R, Siekmeyer G, Urban R, Bourgeois IM. Pulse generator for biomechanical cardiac assistance by counterpulsation technique. In: Chiu RC-J, Bourgeois IM (Eds.). Transformed muscle for cardiac assist and repair. Futura Publishing Company Inc., Mount Kisco, NY 1990:281290.

30. Vd Ree JK. Zimmerman ANE. V Heijst ANP: Intoxication by tricyclic antidepressant drugs. Neth J Med 1977:20;149-153.

31. Lucas CM. Cheriex E. Van der Veen FH, Habets J, Van der Nagel T. Penn OC, Wellens HJ: Imipramine induced heart failure in the dog: A model to study the effect of cardiac assist devices. Cardiovasc Res (in press).

32. Macoviak JA. Stenson EB, Starkey TD, Hansen DE, Cahill PD, Miller DC, Shumway NE: Myoventriculoplasty and neoventricle myograft: cardiac augmentation to establish pulmonary blood flow. J Thorac Cardiovasc Surg 1987; 93:212-220.

33. Macoviak JA, Stephenson LW, Spielman S, Greenspan A, Likoff M, St John Sutton M, Reichek N, Rashkind WJ, Edmunds LW: Replacement of ventricular myocardium with diaphragmatic skeletal muscle: short-term studies. J Thorac Cardiovasc Surg 1981;81:519-527.

34. Molteni L, Almada HE, Ferreira RF, Ortega D: Assessment of the optimal time interval between $\mathrm{QRS}$ and single-pulse stimulation in dynamic cardiomyoplasty. In: Chiu RC-J. Bourgeois IM (Eds.). Transformed muscle for cardiac assist and repair. Futura Publishing Company. Inc. Mount Kisco. NY, 1990;189-196.

35. Almada H. Molteni L, Ferreira R, Ortega D, Cianciulli T, Llanos S, Prezioso H: The value of the Echo-Doppler in cardiomyoplasty procedures. J Cardiac Surg 1991:6(suppl): 113-118. 
36. Levin HR, Tsitlik JE, Halperin HR: Optimization of the timing of skeletal to cardiac muscle contraction during dynamic cardiomyoplasty: Analysis using a mathematical model. J Cardiac Surg 1991;6(suppl):236-244.

37. Sobermann MS, Wornom III IL, Justicz AG, Coleman III JJ, Austin GE, Alazraki NP. Sink JD: Latissimus dorsi dynamic cardiomyoplasty of the right ventricle. $J$ Thorac Cardiovasc Surg 1990;99:817-827.

38. Mannion JD, Velchik M, Hammond R, Alavi A, Mackler T, Duckett S, Staum M, Hurwitz S. Brown W, Stephenson LW: Effects of collateral blood vessel ligation and electrical conditioning on blood flow in dog Latissimus Dorsi muscle. J Surg Res 1989;47:332-340. 
hapter 6

\section{Long-term follow-up after dynamic cardiomyoplasty in goats}

Caroline MHB Lucas

Elisa N Kloosterman - Castro-Ravelo

Frederik $\mathrm{H}$ van der Veen

Emile C Cheriex

Miek G Havenith

Roberto C Lorusso

Jo Habets

Theo van der Nagel

Olaf CKM Penn

Hein JJ Wellens

Submitted for publication. 


\title{
Long-term follow-up after dynamic cardiomyoplasty in goats
}

\begin{abstract}
Dynamic cardiomyoplasty has been introduced as a new method to treat patients with severe cardiac failure. To obtain information on long-term effects on hemodynamics and muscle histology, goats were studied after a dynamic cardiomyoplasty procedure using the left latissimus dorsi (LD) muscle.

Methods and results: Twentyfour goats were studied. Sixteen goats were available for invasive and transesophageal echo doppler hemodynamic mealsurements and histological study at least 12 weeks after the procedure. At that time, only two goats showed significant improvement in left ventricular function both before and after imipramine induced depression of cardiac function. Histological investigation in these 2 goats revealed preservation of muscle structure and almost complete transformation to type I fibres of the wrapped muscle. The remaining 14 goats showed extensive lipomatosis of the LD muscle. Severe intimal hyperplasia and proliferation of smooth muscle cells in the arterial walls was present without increase in capillary-to-fibre ratio. Acetylcholinesterase enzyme staining demonstrated destruction of motor-endplates. An increase in endoneural and endomyseal connective tissue was observed with some goats showing destroyed nerve branches near the electrodes. At the ultrastructural level important distortion of the myofibrillar bundles within the individual muscle cells was seen, together with signs of damage to the mitochondria. These findings differed from those observed following long-term electrical stimulation of the goat latissimus dorsi muscle in situ.

Conclusions: Dynamic cardiomyoplasty is of use in the treatment of severe heart failure, if the histological structure of the wrapped latissimus dorsi muscle remains intact. The current approach used in dynamic cardiomyoplasty may lead to deterioration of the wrapped muscle long-term. Future research should be directed to optimal preservation of $\mathrm{LD}$ muscle structure, in spite of wrapping and continuous
\end{abstract}


electrical stimulation. Areas to be investigated include the most optimal fibre orientation of the wrapped LD muscle and a more prudent stimulation protocol to prevent ischemic damage to the muscle and direct trauma to the nerve branches.

\section{INTRODUCTION}

In the last decade, dynamic cardiomyoplasty has been introduced as a now surgical technique to treat patients with severe heart failure. During this procedure the latissimus dorsi (LD) muscle is wrapped around the heart and electrically stimulated synchronously with cardiac contraction.

Since the first clinical application in 1985 (1), a number of reports have appeared on acute and long-term effects of dynamic cardiomyoplasty in animal experiments and patients (2-13).

This article describes our experience during long-term follow-up in a large number of goats. Hemodynamic results from dynamic cardiomyoplasty will be presented followed by the histological findings in the wrapped and chronically stimulated latissimus dorsi muscle. To study the effect of dissection of the LD muscle with subsequent wrapping and electrical stimulation on the morphological aspects of the muscle, the histopathological lindings will be compared with those obtained from in situ stimulated goat. LD muscles.

\section{MATERIALS AND METHODS}

The experiments were performed according to the 'Guide for the Care and Use of Laboratory Animals' (14).

\section{I: $\quad$ Dynamic Cardiomyoplasty procedure}

Twentyfour goats were used with body weights varying from 28 to $55 \mathrm{~kg}$. Anesthesia was induced intravenously with $15 \mathrm{mg}$ per $\mathrm{kg}$ body weight sodium thiopental (Nesdonal) and, after endotracheal intubation, maintained with oxygen/ nitrous oxide $(1: 2)$ and $1.5 \%$ Fluothane. During the experiments the lungs were ventilated with a positive pressure respirator (Pulmomat) and body temperature was maintained constant with a heating blanket. During the cardiomyoplasty procedure, a continuous infusion of $5 \%$ Ringer's lactate was administered through a peripheral vein. Standard ECG leads 1, Il and III were recorded simultaneously. The whole procedure was performed under sterile conditions and antibiotic cover using Amfipen $1000 \mathrm{mg}$ iv pre-operatively.

\section{Pedicle preparation}

A leftsided midaxillary incision was performed, and the collateral blood vessels to the distal part of the LD muscle were coagulated. All attachments of the muscle were disconnected, except the axillary pedicle to keep the 


\section{2}

thoracodorsal artery, vein and nerve intact. Two intramuscular electrodes (Medtronic SP5528) were implanted in the upper part of the LD muscle flap, perpendicular to the main branches of the thoracodorsal nerve.

Threshold value of stimulation was measured during temporary connection of these electrodes to a programmer (Medtronic SP3028).

\section{Wrapping procedure}

A partial resection of the anterior arch of the second rib was performed to transfer the muscle and pacing leads into the thorax. The thoracic cavity was opened at the fifth left intercostal space and the pericardium removed. A sensing electrode (Medtronic SP5548) was implanted in the left ventricular wall. Subsequently, the left LD muscle was wrapped in a counterclockwise fashion around both ventricles. The muscle was first positioned around the right ventricle and fixed with its lateral side near the atrioventricular groove at the base of the heart with interrupted sutures. This was followed by wrapping the remaining distal part of the muscle around the left ventricle. After wrapping this part, it was sutured to its proximal portion. Because in healthy goats the surface of the LD muscle can cover more than the circumference of both ventricles, the most distal part of the LD muscle was sutured in a double layer on top of the first layer over the anterior wall of both ventricles. All three electrodes were connected to a cardiomyostimulator (SPl005 Medtronic), which was subsequently positioned in the interscapular region.

All goats received Amfipen (1000 $\mathrm{mg}$ im daily) for 5 days as antibiotic treatment and buprenorfine (Temgesic $0.6 \mathrm{mg}$ im daily) for analgesia.

Two weeks after the wrapping procedure electrical stimulation was started using the protocol of Chachques (8). Every two weeks the number of stimuli to the LD muscle was progressively increased. After 10 weeks the stimulation mode consisted of burst pacing (individual pulse width $210 \mu$ seconds, burst duration $185 \mathrm{msec}$, burst frequency $30 \mathrm{~Hz}$ ) with a voltage of 4 volt. One pacing burst was synchronized to every heart beat with an upper limit of 100 LD contractions per minute.

Hemodynamic studies were performed after a follow-up period of at least 12 weeks (the end of the conditioning period of the wrapped LD muscle).

\section{II: In situ latissimus dorsi stimulation}

For this purpose 6 healthy goats were used with body weights ranging from 29 to $54 \mathrm{~kg}$.

The animals were anesthetized initially with sodium thiopental (Nesdonal 15 $\mathrm{mg} / \mathrm{kg}$ body weight) into the jugular vein, intubated and ventilated with oxygen/nitrous oxide (1:2) and $1.5 \%$ Fluothane. A $15 \mathrm{~cm}$ longitudinal skin incision was made in the left midaxillary line under sterile conditions and pre-operative antibiotic cover (Amfipen $1000 \mathrm{mg}$ iv). Subsequently, two intramuscular electrodes (Medtronic SP5528) were attached approximately $6 \mathrm{~cm}$ apart into the proximal part of the LD muscle, perpendicular to the main branches of the left thoracodorsal nerve. An Itrel pulse generator (Medtronic SP7420) was connected with these electrodes and positioned in 
a subcutaneous pocket in the interscapular region. Buprenorfine (Temgesic $0.6 \mathrm{mg} \mathrm{im}$ ) was given for 2 days for analgesia and $1000 \mathrm{mg}$ Amfipen im as antibiotic treatment.

Stimulation of the left LD muscle was started 2 weeks after this operation according to the protocol shown in Table 1, which is comparable to the one commonly used after a dynamic cardiomyoplasty procedure. All individual electrical pulses had a duration of $210 \mu$ seconds. The voltage, which was adjusted to produce an easily palpable and visible contraction of the entire LD muscle without causing apparent discomfort to the animal, measured $2.6 \pm 0.4 \mathrm{~V}$. The muscle was stimulated continuously during 24 hours a day for a period of 12 weeks.

TABLE 1.

Stimulation protocol of in situ LD muscles

\begin{tabular}{lllll}
\hline & week & $\begin{array}{l}\text { number of } \\
\text { pulses }\end{array}$ & $\begin{array}{l}\text { interpulse } \\
\text { interval (msec) }\end{array}$ & bursts/min \\
\hline A: $\mathrm{n}=3$ & $1-2$ & $\mathrm{l}$ & & 50 \\
& $3-4$ & 2 & 100 & 50 \\
& $5-6$ & 3 & 67 & 50 \\
& $7-8$ & 6 & 33 & 50 \\
& $9-10$ & 6 & 33 & 60 \\
& $11-12$ & 6 & 33 & 80 \\
\hline $\mathrm{B}: \mathrm{n}=3$ & $1-2$ & 3 & 33 & 30 \\
& $3-4$ & 3 & 33 & 40 \\
& $5-6$ & 3 & 33 & 50 \\
& $7-8$ & 6 & 33 & 50 \\
& $9-10$ & 6 & 33 & 60 \\
& $11-12$ & 6 & 33 & 80 \\
\hline
\end{tabular}

After this period biopsies were taken from the midportion of stimulated and nonstimulated LD muscles. One biopsy from both sides was quickly frozen in isopentane. A second one was stored in $4 \%$ paraformaldehyde. Also small biopsies for electronmicroscopic evaluation were taken from both muscles. After the 12 week period, all 6 goats were sacrificed using an overdose of thiopental.

\section{A: Hemodynamic Evaluation}

The hemodynamic effects of dynamic cardiomyoplasty were investigated. using both invasive and echo-Doppler measurements. Measurements were made both in the normal heart and after the induction of cardiac failure by imipramine.

Anesthesia, ventilation, infusion and ECG-recordings were performed as described for the wrapping procedure. Aortic blood pressure (AoP) was 


\section{4}

measured with a Millar catheter-tip micromanometer (7F Millar PC470) positioned into the aortic arch through the femoral artery. Left ventricular pressure (LVP) measurements were obtained with a Millar catheter-tip micromanometer (7F Millar PC470), inserted through the right brachial artery into the left ventricle. Left ventricular end-diastolic pressure (LVEdP) was separately recorded by clipping the left ventricular pressure at 20 $\mathrm{mmHg}$ and subsequently amplifying the signal on the recorder. The first derivative (positive and negative LVdP/dtmax) was determined as described by Schaper et al (15). Right ventricular pressure (RVP) was measured using a Millar catheter-tip micromanometer inserted into the right ventricle via the left jugular vein. All catheters were flushed repeatedly with saline.

Aortic blood flow velocity recordings were made using a transesophageal approach. A 64 elements $5 \mathrm{MHz}$ phased-array transducer (HP, Massachusetts) was positioned at the level of the ascending aorta perpendicular to the aortic valvular plane, allowing registration of the blood flow just above the valve using pulsed Doppler. The flow integrals were recorded using a stripchart recorder with a paper speed of $50 \mathrm{~mm} / \mathrm{sec}$ and traced off line on a digitizing tablet (Summasketch $\mathrm{II}^{\mathrm{R}}$ ), using a scientific measurement program (Sigmascan ${ }^{R}$ ).

To stimulate the wrapped muscle, different modes of stimulation were used in the order given in Table 2. Contraction of the LD muscle was synchronized to every third heart beat as long as cardiac frequency remained above 100 beats per minute. When the heart rate was below this value the cardiomyostimulator switched automatically to a synchronization ratio of one LD muscle contraction to every second cardiac contraction. Voltage of stimulation was programmed at 5 volt and subsequently increased to 8 volt when no effect was seen on hemodynamic variables. All pacing modes were applied during at least 2 minutes. Stimulation free intervals of approximately 30 seconds were programmed in between the different protocols.

After finishing this part of the protocol, depression of cardiac function was induced by continuous infusion of imipramine (Tofranil ${ }^{R}$, Ciba-Geigy, AG, Basel. Switzerland) in a dosage of $7.5 \mathrm{mg} / \mathrm{kg}$ bodyweight per hour. Thereafter, the stimulation protocol of the LD muscle was repeated.

\section{Data analysis}

In each goat 10 non-assisted beats were measured, immediately before the start of every mode of the stimulation protocol, and 5 assisted beats thereafter. This was done during continuous artificial respiration as respiratory arrest frequently resulted in ventricular extrasystoles. The same approach was followed after the induction of cardiac depression with imipramine.

\section{Statistical analysis}

The 10 non-stimulated and 5 stimulated beats during every mode of stimulation were averaged for every goat. The mean of the non-assisted beats were subsequently averaged for every stimulation mode (12 in total) and compared using repeated-measures one way analysis of variance. The mean of 
TABLE 2.

Successive stages of the stimulation protocol

\begin{tabular}{lccc}
\hline protocol & $\begin{array}{l}\text { number of pulses } \\
\text { In a burst }\end{array}$ & $\begin{array}{l}\text { interpulse interval } \\
(\mathrm{msec}) / \text { burst frequency } \\
(\mathrm{Hz})\end{array}$ & $\begin{array}{l}\text { sensing delay to the } \\
\text { R-wave } \\
\text { (msec) }\end{array}$ \\
\hline I & 1 & & 4 \\
II & 2 & $100 / 10$ & 4 \\
III & 3 & $67 / 15$ & 4 \\
IV & 6 & $33 / 30$ & 4 \\
V & 10 & $20 / 50$ & 4 \\
VI & 16 & $12 / 85$ & 4 \\
VII & 1 & & 100 \\
VIII & 2 & $100 / 10$ & 100 \\
IX & 3 & $67 / 15$ & 100 \\
X & 6 & $33 / 30$ & 100 \\
XI & 10 & $20 / 50$ & 100 \\
XII & 16 & $12 / 85$ & 100 \\
\hline
\end{tabular}

all control beats was compared with the mean of all stimulated beats using Student's $t$ test for unpaired samples. The same procedure was performed after depression of cardiac function by imipramine. The effect of imipramine was evaluated by comparing the pooled control beats of all stimulation protocols before and after administration of this cardiodepressive drug using Student's t test for unpaired samples. Differences were accepted to be significant at $\mathrm{P}<0.05$. All data are expressed as mean $\pm \mathrm{SD}$.

\section{B: Histopathological Evaluation}

I: $\quad$ Following dynamic cardiomyoplasty

After completion of the last hemodynamic measurements, a transmural biopsy was taken from the midportion of the right nonstimulated LD muscle for histopathological investigation. This specimen was immediately frozen in isopentane, quenched in dry ice and stored at $-70^{\circ} \mathrm{C}$.

Subsequently, the goats were sacrificed with an overdose of sodium thiopental. After opening the thoracic cavity, the heart with the wrapped left LD muscle was carefully excised. Adherent pulmonary tissue, and fatty tissue were removed. Four biopsies from the LD muscle were taken at different locations, starting at the pedicle and descending to a part of the muscle next to the apex of the heart (corresponding to locations A, B, C and D as will be described later). These biopsies were snap frozen in isopentane and stored at $-70^{\circ} \mathrm{C}$ for further analysis. All pieces of tissue were carefully positioned for transverse sectioning. 


\section{6}

Small parts $(1 \times 1 \times 1 \mathrm{~mm})$ from these biopsies were taken and fixed in $2.5 \%$ glutaraldehyde in $0.01 \mathrm{M}$ phosphate buffer at $\mathrm{pH} 7.3$. The osmolality of the solution was adjusted to 330 mosmole by the addition of sucrose for subsequent electronmicroscopic investigation.

Finally, the remaining part of the heart and the wrapped LD muscle were stored in $4 \%$ paraformaldehyde pending subsequent analysis.

\section{Histological analysis}

All specimen frozen in isopentane were used for fibre-typing, capillary counts and the measurement of fibre diameter of type I and type II fibres. This was done by combining immunohistochemical fibre typing with the monoclonal antibody R11D10 and enzyme-histochemical capillary staining using alkaline phosphatase. For this purpose $8 \mu \mathrm{m}$ cryostat sections were submerged in cold acetone $\left(-4^{\circ} \mathrm{C}\right)$ for 5 minutes. Incubation of all sections was performed for 60 minutes in a medium containing nitroblue tetrazolium, 5-bromo-4 chloro-3-indolyl phosphate. After washing these sections 3 times 5 minutes in tris(hydroxymethyl) amino methane buffer saline (TBS), they were incubated at room temperature with the mouse monoclonal antibody R1 1D10 (Centocor Europe), 1:10000 diluted in TBS with 1\% BSA for 45 minutes. This antibody was raised against human left ventricular myosin heavy chain (16) and demonstrates immunoreactivity to type I muscle fibers in human, canine (17) and goat (unpublished observation) skeletal muscle. Sections were washed again in TBS for 3 times 5 minutes, followed by application of 1:200 diluted (in TBS with 1\% BSA) peroxidase labeled rabbit anti-mouse antiserum for 1 hour at room temperature. The peroxidase activity was visualized by diaminobenzidine as chromogen. Finally all sections were stained for 5 minutes using diluted eosine.

Threehunderd muscle fibres were identified immunohistochemically in at random distributed fascicles in all specimen. A Zeiss microscope in connection with a Hitachi FP- 10 camera and an interactive image analysis system (MOP Videoplan Kontron) were used to count the percentage of type I and type II fibres. Furthermore, the cross-sectional area of these individual muscle fibres was measured and expressed as the diameter of the circle of an equivalent area. Also, the number of capillaries per muscle fiber which were adjacent to the muscle fibres used for fibre typing and fibre area measurements were counted.

Slices were also stained for acetylcholinesterase enzyme activity to identify the motor end-plates.

All hearts with wrapped LD muscles, temporarily stored in $4 \%$ paraformaldehyde, were cut in four slices perpendicular to the baso-apical axis, starting at the base of the heart near the atrioventricular groove and ending at the apex. From every slice at least 3 specimen, composed of myocardium with adjacent LD muscle, were taken at different locations. Also from all 24 goats specimen were taken from the tissue around both intramuscular electrodes. All specimen were embedded in paraffin and cut in $3 \mu \mathrm{m}$ sections. For staining hematoxylin-eosin, elastin-von Giesson, Picrosirius 
Red and, the monoclonal antibody R11D10 were used for immunohistochemical staining. Furthermore a number of specimen was stained using periodic acid-Schiff (PAS).

The Picrosirius Red-Polarization method, staining collagen type I and III, was used to demonstrate the amount of endomyseal connective tissue within the left and right LD muscle (18). For this purpose $3 \mu$ meter sections were incubated for 5 minutes in aqueous phosphomolybdic acid (19), followed by staining in $0.1 \%$ sirius red F3BA (C.I. 35780, Polysciences, Norhampton, UK), in satured aqueous picric acid (90 minutes). Subsequently, the sections were washed with $0.01 \mathrm{~N} \mathrm{HCl}$, cleared and mounted. Imaging of the amount of sirius red staining skeletal muscle tissue was performed using an image analyzer (CAS 200, Inc, Becton and Dickinson) with its Quantitative Nuclear Antigen program. Two masks are used in this program. One to define a threshold for the total area of skeletal muscle tissue and another to define the threshold for the measurement of the amount of sirius red staining area, which is expressed as the percentage of the total measured area. Five skeletal muscle fascicles were chosen at random from one specimen at all four slices. The amount of endomyseal connective tissue was measured at 5 different sides within each selected fascicle.

\section{Evaluation of histological slides}

All specimen were evaluated by two individual observers. First, the percentage of type I and type II fibres was calculated, counting 300 fibres per specimen. The amount of connective tissue around the electrodes was checked by measuring the width of the collagen sheath at 10 different sides around every intramuscular electrode in all goats, using the MOP Videoplan Kontron. Subsequently the amount of fatty tissue within the separate muscle fascicles was scored by comparing the specimen stained with elastin-Von Giesson from the wrapped LD muscle with the non-stimulated right LD muscle. The area occupied by fat cells in the stimulated LD muscle was scored semiquantitatively; $1+(0-25 \%) .2+(25-75 \%)$ or $3+(>75 \%)$.

\section{Electron microscopic investigation}

Transverse sections of both left and right LD muscle were stained with lead citrate and uranyl acetate and examined using a Philips 300 electron microscope. To assess the structure of individual LD muscle fibres, 6 areas were selected at the peripheral and 6 at the central part of each muscle fibre and photographed at a final magnification of $14.000 x$.

\section{II: $\quad$ In situ LD stimulation}

As mentioned before, in all 6 goats biopsies were taken from the midportion of left and right LD muscles distal to the intramuscular pacing electrodes. These specimen were handled and evaluated as described for the cardiomyoplasty group. 


\section{8}

\section{Statistical analysis}

Results from left and right LD muscles, both from wrapped goats and in situ stimulated goats, were compared using repeated-measures analysis of variance followed by Student's t test for unpaired samples. Differences were accepted to be significant at $\mathrm{P}<0.05$. Values are presented as mean $\pm \mathrm{SD}$.

\section{RESULTS}

According to the protocol, all goats with the in situ stimulated LD muscles were sacrificed after 12 weeks. In the cardiomyoplasty group, a follow-up of 12 to maximally 35 weeks was reached in 16 goats. Eight goats either died spontaneously or were sacrificed because of infection before the end of the 12 th week, which is the end of the LD muscle conditioning period. Two goats from this group succumbed even before the start of the stimulation protocol.

\section{A: Hemodynamic data}

A complete hemodynamic evaluation was obtained in 13 of the 16 goats which were sacrificed after 12 weeks. Three goats died during the transvenous insertion of the right ventricular catheter, before the stimulation protocol of the wrapped LD muscle was started. As demonstrated in Table 3 , pooling of the data of all stimulation protocols resulted in a small but significant increase in right ventricular pressure, both before and after the infusion of imipramine. The time velocity integrate of aortic flow showed a small significant increase only before the induction of cardiac depression by imipramine. No significant effect was seen on all the other measured hemodynamic parameters. Imipramine itself caused severe depression of left ventricular pressure, right ventricular pressure, aortic pressure and left ventricular $\mathrm{dP} / \mathrm{dt}$, concomitant with a decrease in cardiac frequency from $117 \pm 22$ beats $/ \mathrm{min}$ to $102 \pm 17$ beats/min. The decrease in time velocity integrate of aortic flow appeared less pronounced. Two goats from the group of 13 goats, both with a follow-up of 18 weeks, demonstrated significant increases in pressures and aortic flow before and after the administration of imipramine. Representative tracings from one of these goats are presented in figures 1 and 2 . As will be discussed later in the histopathology section, these excellent hemodynamic results were accompanied by a good preservation of skeletal muscle structure.

\section{B: $\quad$ Histopathological data}

I: $\quad$ The dynamic cardiomyoplasty group

In Table 4 all histological data are presented from the 16 goats, having a follow-up of more than 12 weeks. Electrical stimulation resulted in pronounced transformation to the fatigue-resistant type I fibres in all examined LD muscles. This was accompanied not only by a significant decrease in fibre diameter of type I fibres but also by a large variability in fibre size. 
TABLE 3.

Hemodynarnic results of assisted versus non-assisted beats both before and after the administration of imipramine $(n=13)$.

\begin{tabular}{lllllll}
\hline & $\begin{array}{l}\text { TVI } \\
(\mathrm{cm})\end{array}$ & $\begin{array}{l}\text { RVP } \\
(\mathrm{mmHg})\end{array}$ & $\begin{array}{l}\text { LVEdP } \\
(\mathrm{mmHg})\end{array}$ & $\begin{array}{l}\text { LVP } \\
(\mathrm{mmHg})\end{array}$ & $\begin{array}{l}\text { AoP } \\
(\mathrm{mmHg})\end{array}$ & $\begin{array}{l}\text { LVdP/dlt } \\
\text { (mmHg/sec) }\end{array}$ \\
\hline C & $7.66 \pm 1.51$ & $27 \pm 8$ & $14 \pm 6$ & $110 \pm 27$ & $111 \pm 26$ & $1300 \pm 411$ \\
S & $8.33 \pm 2.07^{*} 29 \pm 9^{*}$ & $14 \pm 7$ & $112 \pm 27$ & $112 \pm 26$ & $1326 \pm 411$ \\
C Imi & $6.50 \pm 1.68^{+}$ & $19 \pm 6^{+}$ & $16 \pm 3^{+}$ & $62 \pm 21^{+}$ & $63 \pm 21$ & $554 \pm 124^{+}$ \\
S Imi & $7.19 \pm 2.44^{*}$ & $21 \pm 7^{*}$ & $16 \pm 3$ & $63 \pm 21$ & $64 \pm 22$ & $576 \pm 146$ \\
\hline
\end{tabular}

Abbreviations: $A O P=$ aortic pressure, $\mathrm{C}=$ non-assisted beats, $\mathrm{C}$ Imi $=$ non-assisted beats after imipramine. LVdP/dt = left ventricular $\mathrm{dP} / \mathrm{dt}, \mathrm{LVP}=$ left ventricular pressure, $\mathrm{RVP}=$ right ventricular pressure, $\mathrm{S}=$ assisted beats, $\mathrm{S}$ Imi = assisted beats after imipramine, TVl = time velocity integrate flow of the ascending aorta. *: significant difference between non-assisted and assisted beats ( $P<0.05),+$ : significant difference between non-assisted beats before and after the infusion of imipramine $(P<0.05)$. All data are expressed as $X \pm$ SD.
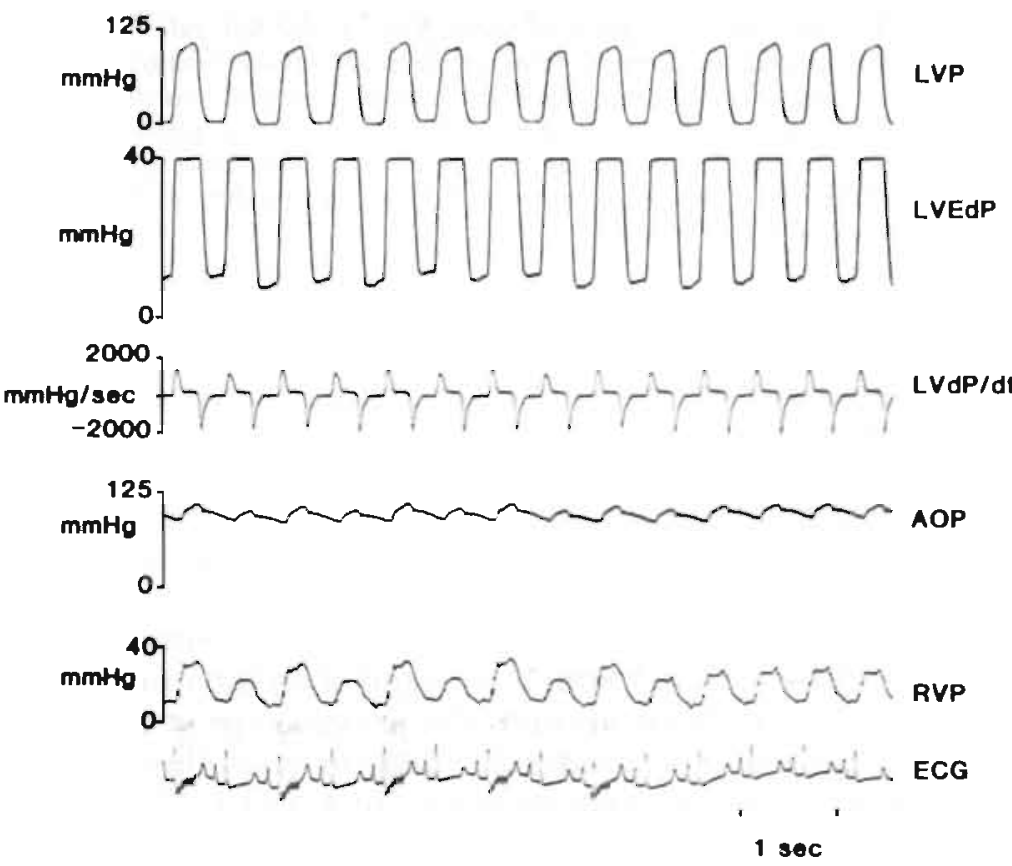

FIGURE 1 .

Registration showing the effect of synchronous burst pacing of the urapped $L D$ muscle on different parameters of cardiac function. Pacing spikes of the stimulator are visualized in the ECG-tracing at the bottom left side of the figure, demonstrating burst stimulation of the wrapped $L D$ muscle synchronized to every other heart beat. Abbreviations: $A O P=$ Aortic pressure. $E C G$. = electrocardiogram, LVP = left ventricular pressure, $L V E d P=$ left ventricular end diastolic: pressure. $L V d P / d t=$ positive and negative $d P / d t$ of the left ventricle. $R V P=$ Right ventriculas pressure. 


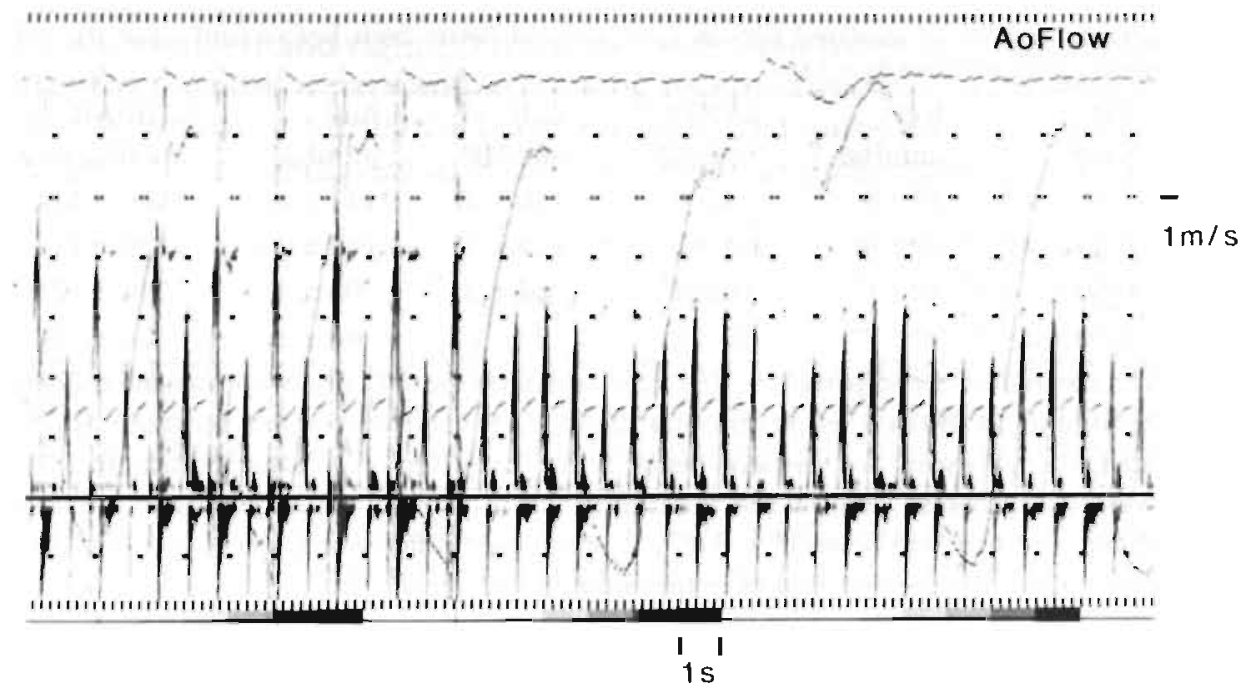

FIGURE 2.

Transesophageal doppler echocardiogram of aortic flow on the left side, demonstrating the increase in aortic flow during stimulation of the wrapped LD muscle, using a burst of 6 pulses at a synchronization ratio of one $L D$ muscle contraction to every other cardiac contraction On the right side, the basal nonassisted aortic flow is displayed. A left ventricular pressure curve is shown concomitant with the flow registration. Respiration is seen as a third curve within the tracing. The electrocardiogram shows the individual pacing spikes during electrical stimulation of the LD muscle.

The percentage of endomyseal connective tissue increased to a maximum of $17.5 \pm 9.0 \%$ in that part of the LD muscle adjacent to the apex of the heart (location D). Although there was a tendency to a greater increase in endomyseal connective tissue, compared to the in situ stimulated muscle, this reached statistical significance only at location $C$ of the wrapped LD muscle. Although this was not quantitatively measured, a clear increase in perimyseal connective tissue was also seen at all locations.

The width of the connective tissue sheath around the intramuscular electrodes measured less than $0.5 \mathrm{~mm}$, if no signs of an inflammatory reaction were visible $(0.37 \pm 0.12 \mathrm{~mm}$ around the proximal $(\mathrm{n}=21)$ and $0.34 \pm$ $0.09 \mathrm{~mm}$ around the distal electrodes $(n=16))$. In case of inflammation, the width of the surrounding collagen increased to $1.53 \pm 0.76 \mathrm{~mm}$ around the proximal $(n=3)$ and $2.67 \pm 0.2 \mathrm{~mm}$ around the distal electrode $(n=8)$.

Contrary to the results of in situ stimulated LD muscles (Table 5) no increase in capillary-to-fibre ratio was found in the wrapped muscles. Moreover, severe intimal hyperplasia and proliferation of smooth muscle cells was seen in the larger arteries in a large number of specimen. A representative example is given in figure 3.

As demonstrated in the fatty tissue score, wrapping and electrical stimulation resulted in 14 of the 16 goats in a marked increase in fat cells both in 
TABLE 4.

Histological data concerning changes in urapped LD muscles stimulated for more than 12 weeks.

\begin{tabular}{llllll}
\hline & right LD & left A & left B & left C & left D \\
\hline \% type I & $27 \pm 7 \#$ & $89 \pm 16^{*}$ & $92 \pm 13^{*}$ & $92 \pm 13^{*}$ & $96 \pm 5^{*}$ \\
Dcir I $(\mu \mathrm{m})$ & $57.8 \pm 6.2$ & $41.8 \pm 10.5^{*} \#$ & $42.1 \pm 9.9^{*} \#$ & $43.7 \pm 9.1^{*}$ & $45.8 \pm 7.1^{*}$ \\
Dcir II $(\mu \mathrm{m})$ & $58.6 \pm 8.2$ & $50.3 \pm 15.1$ & $46.4 \pm 18.7$ & $50.8 \pm 12.9$ & $43.1 \pm 4.2^{*}$ \\
$\%$ area endomys. & $3.7 \pm 1.3$ & $13.3 \pm 5.2^{*}$ & $12.8 \pm 3.5^{*}$ & $15.0 \pm 5.1^{* \#}$ & $17.5 \pm 9.0^{*}$ \\
con.tiss. & & & & & \\
cap/fibre ratio & $1.2 \pm 0.2 \#$ & $1.3 \pm 0.6$ & $1.1 \pm 0.4 \#$ & $0.9 \pm 0.3^{*} \#$ & $1.1 \pm 0.2^{*}$ \\
fatty tissue score (+) & $0 \pm 0$ & $1.8 \pm 1.0^{*} \#$ & $1.7 \pm 1.2^{*} \#$ & $1.6 \pm 1.1^{* \#}$ & $1.6 \pm 1.1^{* \#}$ \\
\hline
\end{tabular}

Left LD A, B. C and D are measurements from biopsies taken at different locations in the wrapped LD muscle starting from the base to the apex of the heart respectively. All data are expressed as mean \pm S.D. $(n=16) ;{ }^{*}$ : significant difference between right and left $L D(P<$ 0.05): \# : significant difference between the in situ stimulated LD group and the cardiomyoplasty group of goats ( $\mathrm{P}<0.05$ )(table 5); Abbreviations: \% type $\mathrm{I}=$ Percentage of type $\mathrm{I}$ muscle fibres. Dcir I = Diameter of a circle equivalent to the measured area of type I muscle fibres, Dcir II = Diameter of a circle equivalent to the measured area of type II muscle fibres. $\%$ area endomys. con. tiss. = Percentage of the LD muscle area occupied by endomyseal connective tissue, cap/fbre ratio = Capillary-to-fibre ratio.

the interfascicular space and in particular within the individual muscle fascicles (figure 4). This increase in adipocytes appeared to be the result of both progressive destruction of myofibrillar bundles followed by replacement with fat and the invasion of fat cells from the outside of the muscle fascicles. This was observed in particular in those LD muscles, which mainly consisted of type 1 muscle fibres. In most of the specimen a great variability in fibre size was seen together with necrotic fibres, whorled fibres, coiled fibres and signs of phagocytosis. Only two goats, both having a follow-up of 18 weeks, showed excellent preservation of LD muscle structure with complete transformation to type I fibres at all biopsies locations, without significant increase in fatty tissue.

In 6 animals electrical stimulation was started after cardiomyoplasty but death occurred at a mean of $6.3 \pm 2.0$ weeks after operation. Biopsies from these animals demonstrated less marked signs of muscle cell destruction and increase in adipocytes as judged by a maximal fatty tissue score of 1.0 \pm 0.8 in location C. An example of one goat of this group is shown in figure 5 , demonstrating an ongoing proces of fibre transformation with a mixture of type I, type II and intermediate fibres. In the 2 goats that died within two weeks after the cardiomyoplasty procedure, no important signs of damage were visible in specimen from the wrapped muscle.

PAS staining showed in a number of biopsies isolated individual fibres with a strong staining reaction as a sign of muscle fibre regeneration.

In the nonstimulated LD muscle, acetylcholinesterase enzyme staining demonstrated clearly defined motor end-plates, restricted to a small area of 


\section{2}

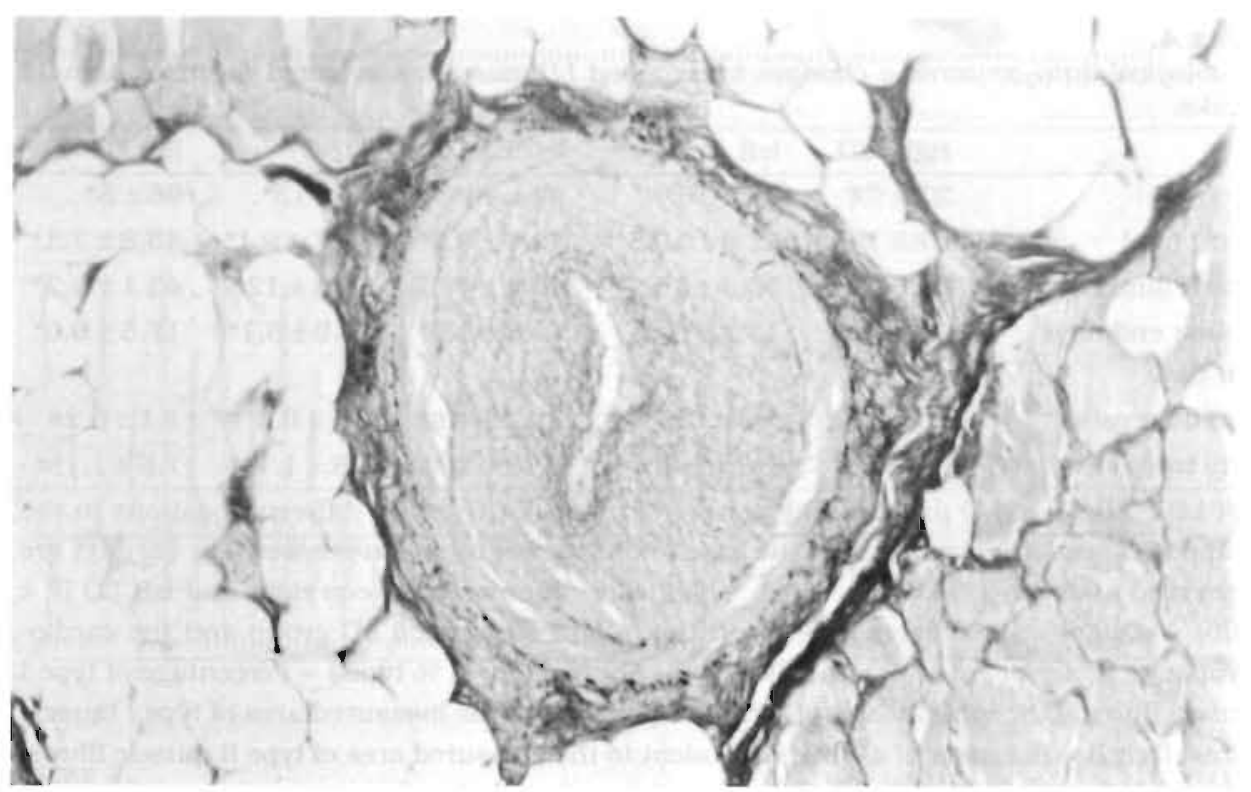

FIGURE 3.

Photograph of a wrapped and long-term stimulated $L D$ muscle demonstrating severe intimal hyperplasia and proliferation of smooth muscle: cells in the arterial wall (Elastin-von Giesson staining).

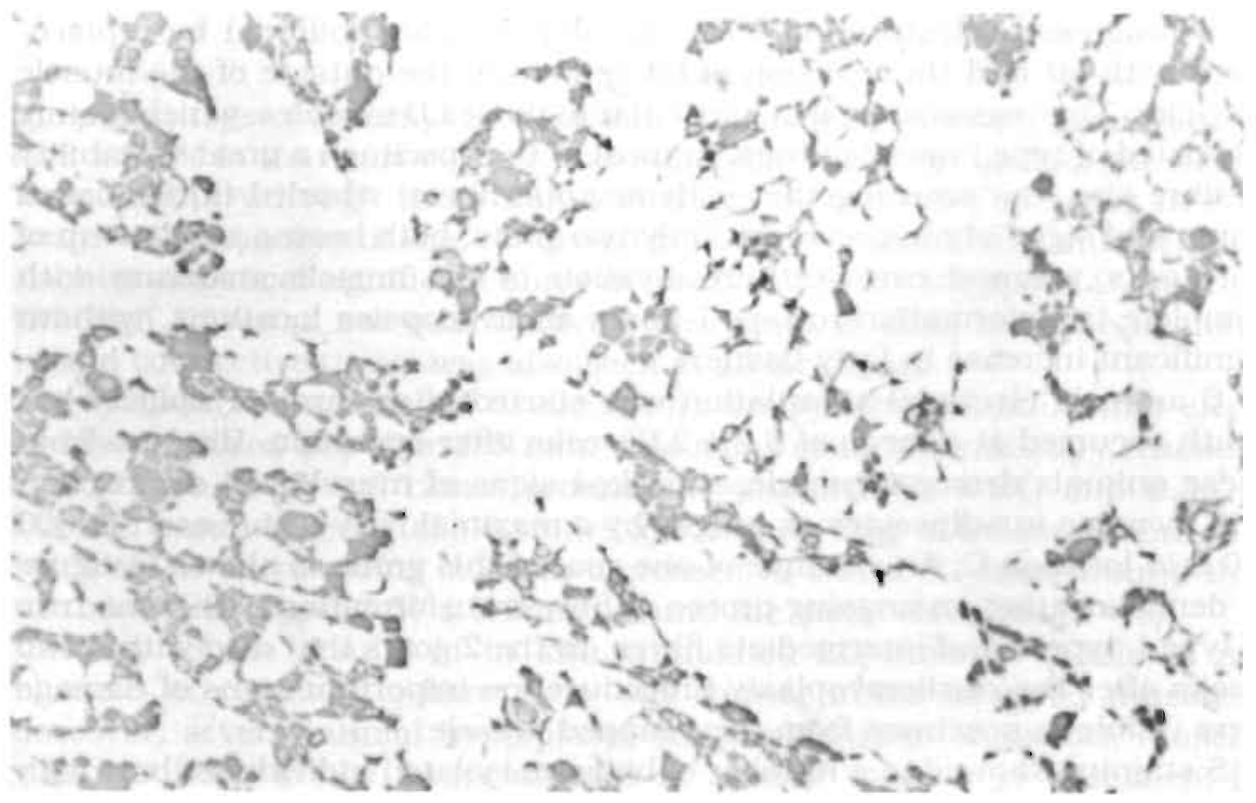

FIGURE 4.

Photograph of a wrapped and long-term stimulated LD muscle demonstrating severe infiltration with and replacement of muscle cells (dark staining) by adipocytes (RIIDIO monoclonal antibody staining). 


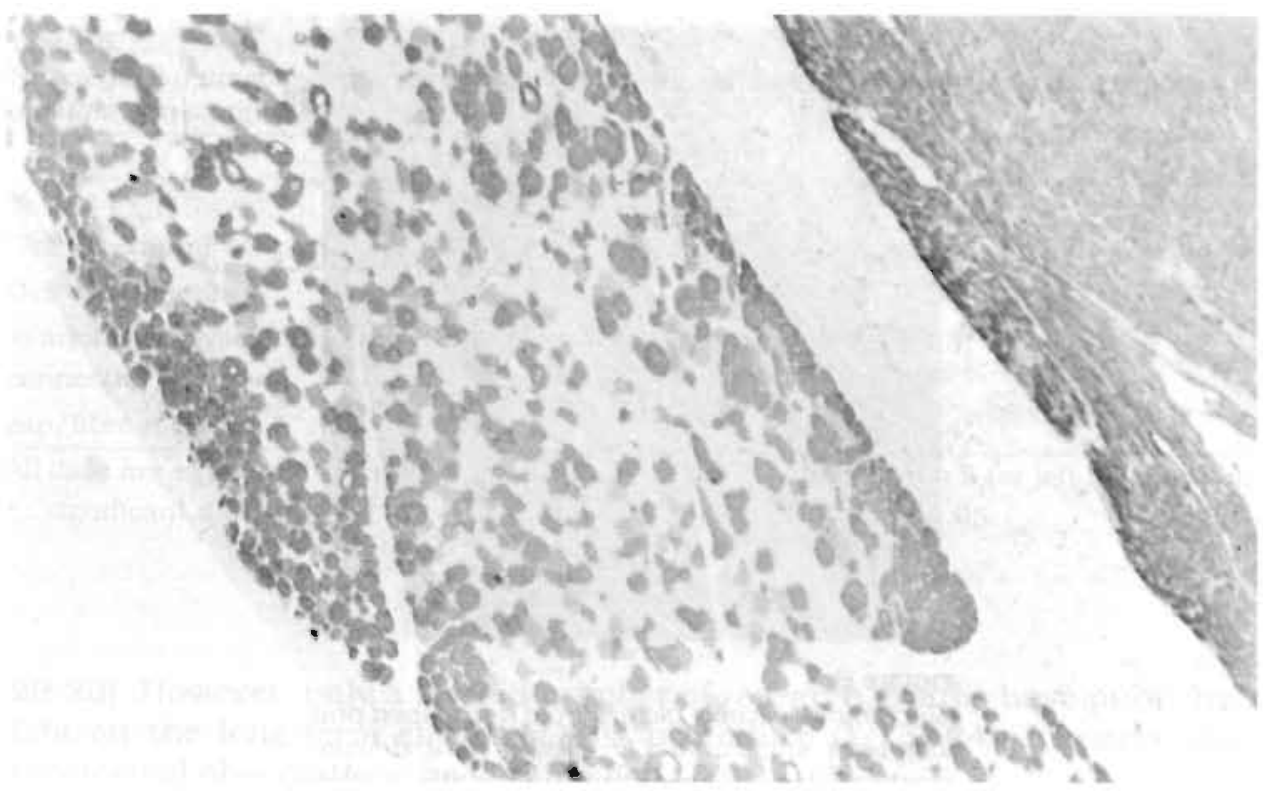

FIGURE 5.

Tissue specimen from a wrapped LD muscle stimulated for 4 weeks demonstrating an ongoing process of muscle fibre transformation from white staining type II fibres to dark staining type I fibres (RI $1 D 10$ monoclonal antibody staining). No signs of skeletal muscle cell destruction are seen.

the muscle cell membrane. However, in the wrapped and stimulated LD muscle, a number of slides demonstrated muscle cells with a diffuse activiy of the enzyme acetylcholinesterase around the entire cell membrane. Also. in the majority of the larger nerves a pronounced increase in endoneural connective tissue was apparent, compared to the nerves in the right nonstimulated LD muscle. In several biopsies signs of damage were seen in the large nerve branches in the vicinity of the intramuscular electrodes.

The contact between myocardium and wrapped LD muscle appeared very heterogeneous. At several sides the muscle was firmly attached to the heart with a small rim of collagen, containing a number of small vessels. At other biopsies sides however the LD muscle was connected very loosely to the heart.

The LD muscles, stimulated for more than 12 weeks demonstrated ultrastructurally severe destruction of myofibrillar bundles, in the majority of muscle fibres with concomitant loss of their original orientation (figure 6). The amount and distribution of mitochondria was very variable and a large number of these mitochondria appeared severely damaged.

\section{II: $\quad$ In situ LD stimulation}

Five out of six in situ stimulated LD muscle demonstrated a histologically normal, vital LD muscle structure mainly composed of type I fibres as 


\section{4}

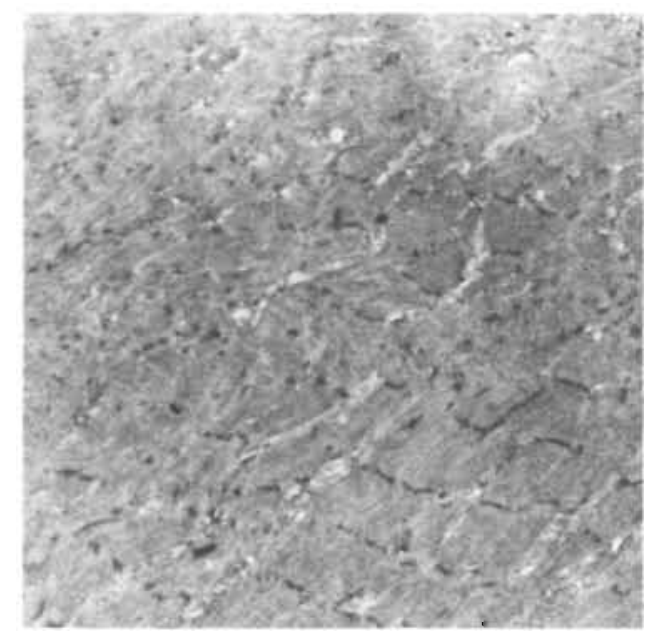

\section{FIGURE 6.}

Electronmicruscopic picture from a wrapped and long-term stimulated $L D$ muscle fibre demonstrating severe disorganization and destruction of the indiridual myofibrillar bundles (magnification $\times 14.000$ ).

demonstrated in Table 5. In the sixth goat a mixture of atrophic fibres next to giant, whorled fibres was seen. All stimulated LD muscles showed a small increase in fatty tissue, which was confined to the interfascicular space.

Stimulation resulted in a significant decrease in fibre diameter of type I fibres and an increase in intrafascicular connective tissue (Table 5). Furthermore, a significant increase in capillary-to-fibre ratio was noticed. No abnormalities were seen in the larger arteries of these muscles. Acetylcholinesterase enzyme staining showed intact motor end-plates in both muscles.

Ultrastructurally well preserved, and well oriented myofibrillar bundles with a significant increase in the number of normal mitochondria per muscle fibre were present.

\section{DISCUSSION}

Dynamic cardiomyoplasty, as a new surgical method to increase depressed cardiac output, has been applied to treat patients in severe cardiac failure. However, as demonstrated by our combined hemodynamic and histopathological data, satisfactory results are only obtained when the wrapped LD muscle remains vital, after dissection and continuous electrical stimulation.

A positive effect of dynamic cardiomyoplasty on hemodynamic function of both healthy and failing hearts has been reported in acute experiments 
TABLE 5.

Histological data concerning changes of the in situ stimulated left LD muscle as compared to the right non-stimulated LD muscle.

\begin{tabular}{lll}
\hline & Right $\mathrm{LD}$ & Left LD \\
\hline \% type I & $19 \pm 4$ & $98 \pm 3^{*}$ \\
Dcir type I $(\mu \mathrm{m})$ & $64.1 \pm 7.9$ & $52.6 \pm 9.5$ \\
Dcir type $\mathrm{II}(\mu \mathrm{m})$ & $63.3 \pm 10.8$ & - \\
\% area endomyseal & $3.2 \pm 2$ & $9.0 \pm 4.3^{*}$ \\
connective tissue & & \\
cap/fibre ratio & $1.0 \pm 0.1$ & $1.8 \pm 0.4^{*}$ \\
\hline
\end{tabular}

All data are expressed as mean $\pm \mathrm{SD}$. ( $n=6$ for right LD muscle. $n=5$ for left LD muscle).

* : significant difference between right and left $L D$ muscle with $P<0.05$.

(20-23). However, only a limited number of research groups have published data on the long-term effects of this procedure (1-13, 24). In particular. histological observations are scarce $(2,3,7,8,10)$.

The positive hemodynamic results in two of our goats demonstrate that, as long as the LD muscle structure is preserved, improvement of cardiac function is possible, both in the normal and failing heart.

Unfortunately, however, our experiments demonstrate severe degenerative changes in LD muscle structure in the majority of our animals, following wrapping and subsequent continuous electrical stimulation. The muscle changes in these animals look similar to those of patients with muscular dystrophy and animal models simulating this disease $(25,26)$. These changes were not seen in the in situ stimulated LD muscles. Although, both the wrapped and in situ stimulated LD muscles showed almost complete transformation to fatigue-resistant type I fibres, which are capable of long-term continuous contraction, these fibres were progressively replaced by fat cells in the majority of the wrapped LD muscles. Moreover, an increase in endomyseal connective tissue was seen, which tended to be more pronounced compared to the in situ stimulated muscle.

As reviewed by Salmons and Henriksson, in situ stimulation of the LD muscle can be performed without detrimental effect on muscle structure (27). This is underlined by our data. Recently Mayne and coworkers (28) showed that the canine LD muscle showed no damage, even after one year of continuous electrical stimulation in situ. This suggests that deterioration of our goat LD muscle structure after a cardiomyoplasty procedure probably results from the distal dissection, followed by the wrapping around the heart. Subsequent electrical stimulation may have played an additive role in the progressive destruction of these LD muscle fibres. Comparable negative effects on skeletal muscle structure have also been described in case of the application of transposed and electrically stimulated canine sartorius muscle, used as a neosphincter around an intestinal loop (29). 


\section{6}

One can only speculate on possible causes for the observed destruction of LD muscle structure.

Firstly, the possibility of direct injury to the muscle during the surgical procedure. In that case however, one would expect signs of destruction of muscle fibres at an earlier phase during the conditioning period, possibly followed by regeneration of muscle cells. Our histological data from a small number of goats, sacrificed soon after the wrapping procedure do not support that mechanism. There were signs of regeneration of individual muscle fibres, as shown by the PAS-staining in our goats but this appeared not to be sufficient to keep a larger part of the muscle vital. Guelinckx et al (30) demonstrated that handling of a transplanted rabbit rectus femoris muscle was the cause for damage to muscle parts next to the repaired tendons. If direct trauma is responsible for damage in the transpositioned LD muscle, one would suspect it to be more pronounced in the distal part of the LD muscle, as this is more intensely handled and distorted during the operation compared to the pedicle. This was not the case in our goats. Also no damage was seen in the in situ LD muscle at the site of the inserted intramuscular electrodes, in spite of the fact that this part of the muscle is handled during electrode implantation.

Secondly, ischemia of the LD muscle could be responsible for the deterioration of muscle structure. As demonstrated no increase in capillary-to-fibre ratio was seen in the wrapped and stimulated LD muscles in contrast to a significant rise in the in situ stimulated muscles. Hudlicka (31) also demonstrated a significant increase in capillary-to-fibre ratio in normally perfused in situ stimulated tibialis anterior and extensor digitorum muscle in rats. Muscles with limited blood supply however, failed to demonstrate an increase in capillary-to-fibre ratio, together with a significantly lesser increase in blood flow during stimulation. Severe loss of muscle mass and atrophy of the muscle fibres was seen in these animals, together with partial necrosis of the central part of the muscles. Based on these observations, they considered the increased blood flow in chronically stimulated muscles as the main factor responsible for the increase in capillary-to-fibre ratio. Thus blood flow in the majority of the wrapped LD muscles seemed to become impaired. This is supported by the observation of severe abnormalities in a large number of larger arteries in the LD muscles. Again, one can only speculate about possible causes for this. Although precaution was taken to create a window in the left thoracic wall by resecting a part of the second rib seemingly sufficiently large for the muscle pedicle, kinking of the thoracodorsal artery at the entrance of the left LD muscle in the thorax may have occurred, leading to a reduction in blood supply. Furthermore, a direct effect from the electrical current on the vessel wall of the larger arteries can not be excluded. Already in 1979, Betz et al described the proliferation of smooth muscle cells in the arterial wall following electrical stimulation (32). That these vessel abnormalities were not seen in the in situ stimulated group could have been due to the lower voltage used in this group. Another possibility for the negative effect on blood supply could be the stimulation protocol which is used in fully transformed LD muscles after the condition- 
ing period of 12 weeks. According to this protocol, pacing of the muscles is finally done with a burst of $185 \mathrm{~ms}$ and a burst frequency of $30 \mathrm{~Hz}$ which is synchronized to every heart beat. The maximal number of LD muscle contractions is programmed at $100 / \mathrm{min}$. We have found (see Chapter 8 ) that this number of LD contractions per minute is to high to allow sufficient time for relaxation, thus possibly hampering LD muscle blood flow. This in particular holds true for the fully transformed LD muscle, which is almost entirely composed of slowly contracting and relaxing type I muscle fibres. Geddes et al also described that there is an optimum stimulation rate for maximal muscle blood flow in a paced canine $\mathrm{LD}$ and rectus abdominis muscle (33). Pacing the muscle above this rate resulted in a decrease in muscle blood flow. The publications by Barclay (34) and Gorman et al (35) stress the necessity of paying attention to maximal blood supply to the LD muscle, because of the effect of blood flow on fatigue and force and the occurrence of severe skeletal muscle 'stunning' after 30 minutes of hypoperfusion. The possibility that electrical stimulation of the wrapped LD muscle may add to muscle ischemia is underlined by Hanzlikova and Gutmann (36). They describe complete restoration of contractile and histochemical properties of rat soleus muscle after a thirty days period of complete interruption of main arterial and collateral blood supply. These muscles were not subjected to electrical stimulation as is the case in dynamic cardiomyoplasty. Guelinckx also demonstrated that transposition without electrical stimulation of the rabbit $\mathrm{LD}$ muscle to the thoracic wall with an intact neurovascular pedicle was possible without detrimental effects to the LD muscle structure (37).

Ischemia could also have been responsible for the destruction of nerve branches resulting in a diffuse spread of acetylcholinesterase enzyme-activity along the whole cell membrane. Subsequent denervation could have led to the decay of a large number of muscle fibers with subsequent replacement by fat cells, as described by Schmalbruch (26).

Another possible explanation for the appearance of signs of denervation could be direct trauma to the large nerve branches in the pedicle near the intramuscular electrodes, concomitant with an increase in endoneural connective tissue. Again the absence of these signs of damage in the in situ stimulated LD muscles could be attributed to the lower voltage of pacing. As a fourth cause of LD muscle degeneration one has to consider the transposition of the distal part of the muscle around the heart. This manoeuver results in an orientation of the individual muscle fibres which is quite different from the original orientation. Our electronmicroscopic photographs underline this point as they demonstrate complete loss of orientation, even of individual myofibrils within the separate muscle cells. This loss of orientation could also account for the histological differences between our LD muscles wrapped around the heart and LD muscles used in the so-called skeletal muscle ventricle (38). In the latter application the original direction of the muscle fibres is preserved in a more optimal way. A better preserved fibre direction and thus preservation of LD muscle structure could also explain the contrasting long-term hemodynamic results obtained by Kao et 


\section{8}

al with canine LD muscles wrapped in two different ways around the heart (17). Fortunately, in patients the LD muscle appears better orientated along its original fibre direction after wrapping.

Finally, loss of original stretch of the muscle when transposed around the heart can play a role in the loss of muscle vitality. This results in an increase in connective tissue (39) which can be prevented by electrical stimulation according to Goldspink et al (40). Unfortunately, this preventive effect was not seen in our wrapped goat LD muscles. Rademecker et al demonstrated that loss of stretch of in situ stimulated goat LD muscle results in infiltration of histiocytic cells and fat substitution of myofibrillar bundles (41). Stretch of a skeletal muscle results first of all in hypertrophy of the fibres and an increase in mass (42). This is beneficial as continuous electrical stimulation results in a decrease in those parameters. Stretch also produces a positive effect on muscle blood flow (43). However, 'to much' stretch of the wrapped LD muscle could lead to impairment of cardiac filling. The optimal amount of stretch still remains one of the problems that has to be solved in cardiomyoplasty.

\section{Conclusions}

Our observations on dynamic cardiomyoplasty in goats with a follow-up of more than 12 weeks show that support of both normal and depressed cardiac function can be obtained as long as the wrapped and electrically stimulated LD muscle remains vital. These observations stress the necessity for further research to elucidate and possibly eliminate the factors responsible for the progressive deterioration of the transposed muscle. Such research should, in our opinion, among others concentrate on the optimal surgical technique as to stretch and fibre orientation of the transposed muscle, and the best stimulation protocol after wrapping. Finally damage to the nerve branches should be avoided. 


\section{REFERENCES}

1. Carpentier A. Chachques JC: Myocardial substitution with a stimulated skeletal muscle: First successful clinical case (Letter) Lancet 1985:1:1267.

2. Rossi MA. Braile DM. Souza DRS, Santos JLV, Thevenard RS. Pinto GH, Suzigan S. Grecco O: Dynamic cardiomyoplasty in chronic Chagas' heart disease: Clinicopathological data. Ann Thorac Surg 1991:51:649-651.

3. Magovern GJ, Heckler FR, Park SB, Christlieb IY, Liebler GA, Burkholder JA, Maher TD, Benckart DH, Magovern Jr GJ, Kao RL: Paced skeletal muscle for dynamic cardiomyoplasty. Ann Thorac Surg 1988:45:614-619.

4. Molteni L, Almada H, Ferreira R: Synchronously stimulated skeletal muscle graft for left ventricular assistance. J Thorac Cardiovasc Surg 1989;97:439-446.

5. Lange R, Sack FU, Saggau W, De Simone R. Hagl S: Influence of cardiac function on the performance of the heart/skeletal muscle complex in dynamic cardiomyoplasty. In: D’Alessandro LC (Ed). Heart Surgery 1991. Casa Editrice Scientifica Internationale 1991;559-572.

6. Moreira LFP, Stolf NAG, Bocchi EA. Pereira-Barretto AC. Meneghetti JC. Giorgi MCP. Moraes AV, Leite JJ, da Luz PL, Jatene AD: Latissimus dorsi cardiomyoplasty in the treatment of patients with dilated cardiomyopathy. Circulation 1990;82 (Suppl IV):257-263.

7. Kao RL. Christlieb IY, Magovern GJ. Park SB. Magovern Jr GJ: The importance of skeletal muscle fiber orientation for dynamic cardiomyoplasty. J Thor:ir Cardiovasc Surg 1990;99: 134-140.

8. Chachques JC, Grandjean P. Schwartz K. Mihaileanu S, Fardeau M, Swynghedauw B, Fontaliran F, Romero N, Wisnewsky C. Perier P, Chauvaud S. Bourgeois I, Carpentier A: Effect of latissimus dorsi dynamic cardiomyoplasty on ventricular function. Circulation 1988;78 (Suppl III):203-216.

9. Carpentier A, Chachques JC: Latissimus dorsi cardiomyoplasty to increase cardiac output. In Rabago G, Cooley DA (Eds). Heart valve replacement: Current status and future trends. Futura Publishing Company, Inc., Mount Kisco, NY 1987:473-486.

10. Anderson WA. Andersen JS. Acker MA. Hammond RL, Chin AJ, Douglas PS. Khalafalla AS, Salmons S, Stephenson LW: Skeletal muscle grafts applied to the heart: A word of caution. Circulation 1988:78 (Suppl III): 180-190.

11. Chagas ACP, Moreira LFP, da Luz PL, Camarano GP, Leirner A. Stolf NAG, Jatene AD: Stimulated preconditioned skeletal muscle cardiomyoplasty: An effective means of cardiac assist. Circulation 1989;80 (Suppl IIl):202-208.

12. Moreira LFP, Seferian P, Edimar A: Survival improvement with dynamic cardiomyoplasty in patients with dilated cardiomyopathy. Circulation 1990:82 (suppl III):713.

13. Almada H, Molteni L, Ferreira R, Ortega D, Cianciulli T, Llanos S. Prezioso H: The value of the Echo-Doppler in cardiomyoplasty procedures. J Cardiac Surg 1991;6 (Suppl);113-118.

14. National Academy of Sciences. Guide for the care and use of laboratory animals. DHHS publication No NIH 85-23, revised 1985.

15. Schaper W, Lewi $P$, Jageneau AHM: The determination of the rate of change of the left ventricular pressure (dP/dt). Arch Kreislaufforsch 1965:46:27-41.

16. Khaw BA, Mattis JA. Melincoff G, Straus HW, Gold HK. Haber E: Monoclonal antibody to cardiac myosin: Imaging of experimental myocardial infarction. Hybridoma 1984;3:11-23. 


\section{0}

17. Havenith MG, Visser R, Schrijvers - van Schendel JM, Bosman FT: Muscle fiber typing in routinely processed skeletal muscle with monoclonal antibodies. Histochemistry 1990;93:497-499.

18. Junquira LCU, Montes GS. Sanchez EM: The influence of tissue section thickness on the study of collagen by the picrosirius-Polarization method. Histochemistry 1982;74:153-156.

19. Dolber PC. Spach MS: Picrosirius red staining of cardiac muscle following phosphomolybdic acid treatment. Stain Technol 1987;62:23-26.

20. Biomechanical cardiac assist: Cardiomyoplasty and muscle-powered devices. Chiu RC-J (Ed.). Futura Publishing Company, Inc. Mount Kisco. NY 1986.

21. Transformed muscle for cardlac assist and repair. Chiu RC-J, Bourgeois IM (Eds.). Futura Publishing Company, Inc. Mount Kisco, NY 1990.

22. Cardiomyoplasty. Carpentier A, Chachques JC, Grandjean P (Eds.). Futura Publishing Company Inc., Mount Kisco, NY 1991.

23. J Cardiac Surg 6 (suppl); 1991, Stephenson LW (Ed.), Futura Publishing Company Inc., Mount Kisco, NY.

24. Jatene AD. Moreira LFP. Stolf NAG. Bocchi EA. Seferian P. Fernandes PMP. Abensur $\mathrm{H}$ : Left ventricular function changes after cardiomyoplasty in patients with dilated cardiomyoplasty. J Thorac Cardiovasc Surg 1991;102;132-139.

25. Dubowitz V. Muscle Biopsy: A practical approach. Second edition 1985. Bailliere Tindall, WB Saunders. 289-404.

26. Schmalbruch $\mathrm{H}$ : The effect of peripheral nerve injury on immature motor and sensory neurons and on muscle fibres. Rev Neurol 1988; 144;721-729.

27. Salmons S, Henriksson J: The adaptive response of skeletal muscle to increased use. Muscle \& Nerve 1981:4:94-105.

28. Mayne CN. Anderson WA. Hammond RL. Eisenberg BR, Stephenson LW, Salmons S: Correlates of fatigue resistance in canine skeletal muscle stimulated electrically for up to one year. Am J Physiol 1991;261:C259-C270.

29. Hallan RI, Williams NS, Hutton MRE, Scott M, Pilot MA, Swash M, Koeze TH, Watkins ES: Electrically stimulated sartorius neosphincter: Canine model of activation and skeletal muscle transformation. Br J Surg 1990;77:208-213.

30. Guelinckx PJ, Faulkner JA, Essig DA: Neurovascular-anastomosed muscle grafts in rabbits: Functional deficits result from tendon repair. Muscle \& Nerve 1988; $11: 745-751$.

31. Hudlicka O. Price S: The role of blood flow and/or muscle hypoxia in capillary growth in chronically stimulated fast muscles. Pflügers Arch 1990;417:67-72.

32. Betz $\mathrm{E}$. Schlote $\mathrm{W}$ : Responses of vessel walls to chronically applied electrical stimuli. Basic Res Cardiol 1979;74;10-20.

33. Geddes LA, Badylak SF, Wessale JL, Janas W, Bourland JD, Tacker WA, Stevens L: The use of electrically stimulated skeletal muscle to pump blood. PACE 1990:13:344-362.

34. Barclay JK: A delivery-independent blood flow effect on skeletal muscle fatigue. J Appl Physiol 1986;61:1084-1090.

35. Gorman MW. Barclay JK. Sparks HV: Effects of ischemia on $\mathrm{VO}_{2}$, tension, and vascular resistance in contracting canine skeletal muscle. J Appl Physiol 1988; 65:1075-1081.

36. Hanzlikova V, Gutmann E: Effect of ischemia on contractile and histochemical properties of the rat soleus muscle. Pflügers Arch 1979;379:209-214.

37. Guelinckx PJ: Free microneurovascular muscle grafts: Deficits resulting from transplantation and speculations for myocardial repair. J Cardiac Surg 1991;6 (suppl); 190-194. 
38. Anderson DR, Pochettino A, Hammond RL, Hohenhaus E, Spanta AD, Bridges CR, Lavine S, Bhan RD, Colson M, Stephenson LW: Autogenously lined skeletal muscle ventricles in circulation. J Thorac Cardiovasc Surg 1991;101:661-670.

39. Williams PE. Goldspink G: Connective tissue changes in immobilized muscle. J Anat 1984:138:343-350.

40. Wulliams PE. Catanese T, Lucey EG, Goldspink G: The Importance of stretch and contractile activity in the prevention of connective tissue accumulation. J Anat 1988; 158:109-114.

41. Radermecker MA, Sluse FE, Focant B, Reznik M, Fourny J, Limet R: Influence of tension reduction and peripheral dissection on histologic. biochernical and bioenergetic profiles, and kinetics of skeletal muscle fast-to-slow transformation. J Cardiac Surg 1991;6 (suppl): 195-203.

42. DF Goldspink, Easton J, Winterburn SK, Williams PE, Goldspink GE: The role of passive stretch and repetitive electrical stimulation in preventing skeletal muscle atrophy while reprogramming gene expression to improve fatigue resistance. J Cardiac Surg 1991;6 (suppl);2 18-224.

43. Leighton WD, Russell RC. Feller AM, Eriksson E, Mathur A, Zook EG: Experimental pretransfer expansion of free-flap donor sites: II Physiology, histology and clinical correlation. Plast Reconstr Surg 1988;82:76-82. 
hapter 7

\section{What is the ideal pulse frequency for skeletal muscle stimulation after cardiomyoplasty?}

Caroline MHB Lucas

Frederik $\mathrm{H}$ van der Veen

Pierre A Grandjean

Olaf CKM Penn

Hein JJ Wellens

Published in PACE 1991;14:778-782. 


\title{
What is the ideal pulse frequency for skeletal muscle stimulation after cardiomyoplasty?
}

\begin{abstract}
Routinely, the latissimus dorsi (LD) muscle is stimulated with bursts of pulses at 30 pulses/sec after cardiomyoplasty, to assist the failing heart. At a lower pulse frequency the contractile force decreases and at higher frequencies. the energy demand of the pacemaker increases rapidly. We investigated the effect of the stimulus frequency variation on contractile force in untrained LD muscles and in muscles after 12 weeks of continuous cyclic electrical stimulation. In $6 \mathrm{dogs}$, two electrodes (Medtronic SP 5528) were implanted in the left LD muscle, together with an Itrel muscle stimulator. The LD muscle was stimulated with 30 pulses/sec in bursts to deliver initially 30 and. after 10 weeks, 80 contractions per minute. Both before and after training of the LD muscle, maximum force was observed by stimulating with a frequency of 36 to 130 pulses/sec in a burst. However, training induced a shift in the steep part of the force-frequency relation to lower frequencies. In particular at 15 pulses $/ \mathrm{sec}$, already $60 \%$ of the maximal force was obtained in contrast to $40 \%$ before training. A fatigue test of 8 minutes duration was performed specified by 100 bursts/minute and a burst duration of $0.25 \mathrm{sec}$ at pulse frequencies of $30,36,50$ and 85 pulses/sec. The contractile force decreased significantly during the course of the test at all frequencies. However, the force obtained with 30 pulses/sec stimulation was lower during the initial phase and approximately $10 \%$ higher at the end of the fatigue test, as compared to 36. 50 and 85 pulses/sec stimulation. We conclude that, when the LD muscle is demanded to assist a failing heart by contracting 100 times a minute, pulse trains with a frequency of 30 pulses/sec deliver a near maximal contractile force $(80 \%)$ and are significantly less fatiguing in
\end{abstract}


comparison to pulse trains at higher frequencies. This indicates that LD muscle stimulation after cardiomyoplasty should be performed at 30 pulses/sec.

\section{INTRODUCTION}

In patients in whom the latissimus dorsi (LD) muscle is used for cardiomyoplasty, the muscle is usually stimulated at 30 pulses/sec (1). Stimulation at a lower frequency is less strenuous to the LD muscle, increases longevity of the pacemaker but decreases contractile force. Although 30 pulses/sec stimulation in $0.25 \mathrm{sec}$. bursts will generate a near maximal Force, the quantitative effect of varying the stimulation frequency is not well documented. Ferguson and coworkers (2) measured muscle tension of the tibialis anterior muscle in cats and observed a $32 \%$ increase at 30 pulses/sec stimulation as compared to $10 \mathrm{pulses} / \mathrm{sec}$ stimulation. Anderson and colleagues (3) reported a higher blood pressure when a LD muscle ventricle in the dog was stimulated at 85 pulses/sec, as compared to 25 pulses/sec. These findings indicate submaximal muscle contraction at frequencies of 10 pulses/sec and 25 pulses/sec, which is in line with the report of Hennig and Lomo (4). They observed force summation above 30 pulses/sec in a relatively fast skeletal muscle.

Earlier reports have shown that training gradually changes the LD muscle into a fatigue-resistant, slow muscle $(1,5,6)$. Whether the transformed muscle should also be stimulated at 30 pulses/sec depends largely on contractile force at lower frequencies (7). Long-term stimulation of a fast muscle in cats revealed that the force (percentage of maximum force) nearly doubled at 30 pulses/sec (8). These experiments further showed that a clear decline in contractile force was observed below 20 pulses/sec, which suggests that there is a lower frequency limit in the trained muscle. The lifetime of implantable pulse train stimulators is mostly affected by: a) number of bursts per minute, b) pulse amplitude (V), c) pulse duration, d) lead impedance, and e) number of pulses in a burst (set by the stimulation [requency and the burst duration). Among others, both pulse amplitude and stimulation frequency have a linear relationship with longevity: as voltage and number of pulses increase, the electronic current drain increases. resulting in decreased device longevity.

We therefore studied the eflect of frequency variation on the contractile force and fatigue-resistance of the LD muscle (in situ) in dogs, before and after a training period of 12 weeks.

\section{MATERIALS AND METHODS}

Two stimulation electrodes (Medtronic Model SP5528) were implanted into the left LD muscle and connected to an Itrel muscle stimulator (Medtronic Model 7420) in 6 dogs. About 2 weeks later muscle stimulation was started. 


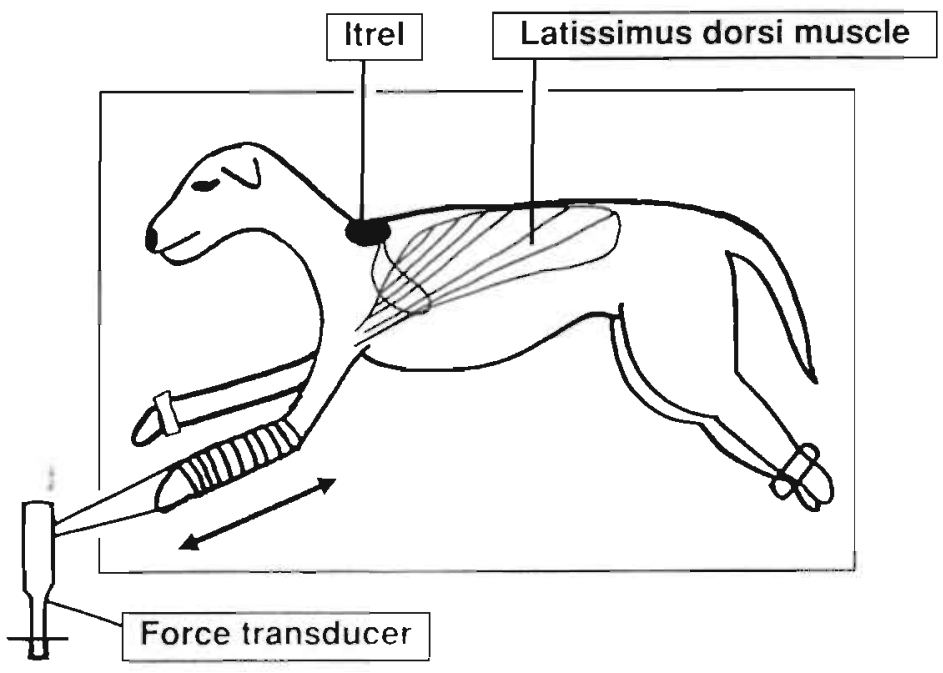

FIGURE 1.

Position of the dog during in situ contraction measurements to determine contractile force and fatigue-resistance of the left $L D$ muscle.

using bursts of stimuli (burst duration $0.25 \mathrm{sec} ; 30$ pulses/sec) with pulses of $210 \mu$ seconds resulting in 8 stimuli per burst. Initially, the interval between two bursts was set at 1.75 seconds and this interval was shortened every 2 weeks to a minimum of 0.52 seconds. This resulted in $30,40,50$, 60,70 and 80 contractions per minute respectively. The LD muscle was stimulated 24 hours a day.

\section{Mechanical tests}

LD muscle contractile force and fatigue resistance were tested prior to and at the end of the training period of 12 weeks. Dogs were premedicated with $0.15 \mathrm{ml} / \mathrm{kg}$ of a mixture containing $10 \mathrm{mg}$ oxycodon $\mathrm{HCl}, 1 \mathrm{mg}$ of acepromazine and $0.5 \mathrm{mg}$ of atropine sulphate per $\mathrm{ml}$, and pentobarbital $15-7$ $\mathrm{mg} / \mathrm{kg}$ iv). Figure 1 shows a dog placed on the right side and the left front leg connected to a pre-calibrated force displacement transducer (Grass FT 10) as reported earlier (9). Subsequently, the muscle was stimulated during one second with rest intervals of 8 seconds at a frequency range from 2 to 130 pulses/sec, to determine contractile force.

A fatigue test was performed during 8 minutes at 100 contractions per minute with a burst duration of 0.25 seconds at $30,36,50$ and 85 pulses/sec. The contractile force was measured every 30 seconds.

\section{LD biopsies and histology}

The animals were anesthetized routinely and the left LD muscle was exposed through a small skin incision.

Transmural biopsies weighing about $0.5 \mathrm{~g}$ were gently removed from the 
muscle and cut in both transverse and longitudinal sections. Biopsies were obtained every 4 weeks and fibre typing was performed using monoclonal antibodies, as described by Havenith and coworkers (10).

\section{RESULTS}

During LD muscle training the fibre composition gradually changed from initially $23 \%$ to $69 \%$ type I fibres after 12 weeks (table 1 ). The force-frequency relation of the untrained muscle showed peak forces at about 85 pulses/sec (figure 2). Using 2 pulses/sec stimulation only $30 \%$ of the peak force was obtained. However, at 30 pulses/sec nearly $80 \%$ of the peak force was observed during contraction. Contractile force of the trained muscle was significantly increased during stimulation at 15 and 20 pulses/sec, as compared to the untrained muscle.

During the 30 pulses/sec fatigue test, contractile force gradually decreased to $79 \%$ of the initial force after 8 minutes (figure 3 ).

Fatigue tests at higher frequencies showed a significant higher force of contraction at the start of the test $\{118 \pm 7 \%$ of contractile force at 36 pulses/sec; mean $\pm \mathrm{SE}$ ). However, contractile force was lower at the end of the fatigue-test, when the muscle was stimulated at 36,50 and 85 pulses/sec, as compared to 30 pulses/sec. The extent of the fatigue during this test is demonstrated in Table 2 , showing the decrease in force at different frequencies as a percentage of the initial force.

\section{TABLE 1 .}

Percentage of type I fibres in latissimus dorsi muscle biopsy spectmens as determined by monoclonal antibodies $(n=6$; mean $\pm S E$ ).

\begin{tabular}{ll}
\hline week nr. & \% type I fibres \\
\hline 0 & $23 \pm 1$ \\
4 & $45 \pm 4$ \\
8 & $61 \pm 6$ \\
12 & $69 \pm 6$ \\
\hline
\end{tabular}

TABLE 2.

Percentage decrease in force at the end of an 8 minutes fatigue test.

\begin{tabular}{lllll}
\hline Pulses $/ \mathrm{sec}$ & 30 & 36 & 50 & 85 \\
$\%$ & $21 \pm 2$ & $35 \pm 9$ & $39 \pm 8$ & $36 \pm 7$ \\
\hline
\end{tabular}

Values are expressed as mean $\pm \mathrm{SE}$. 
128

-+ pre-

- - post-

conditioning

conditioning

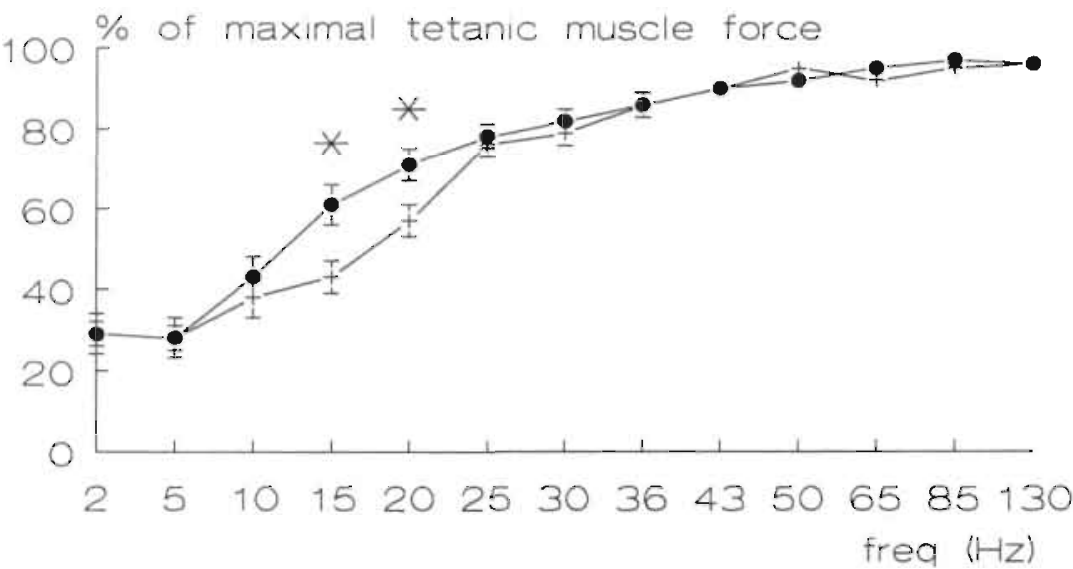

Figure 2.

Force of contractlon during test stimulation with bursts of one second at increasing frequencies (2-130 pulses/sec), before and after 12 weeks of stimulation (values are mean \pm SE). * significant difference of the force (\%) between the conditioned and the unconditioned latissimus dorsi muscle at 15 and 20 pulses/sec.

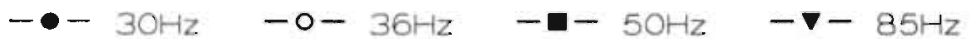

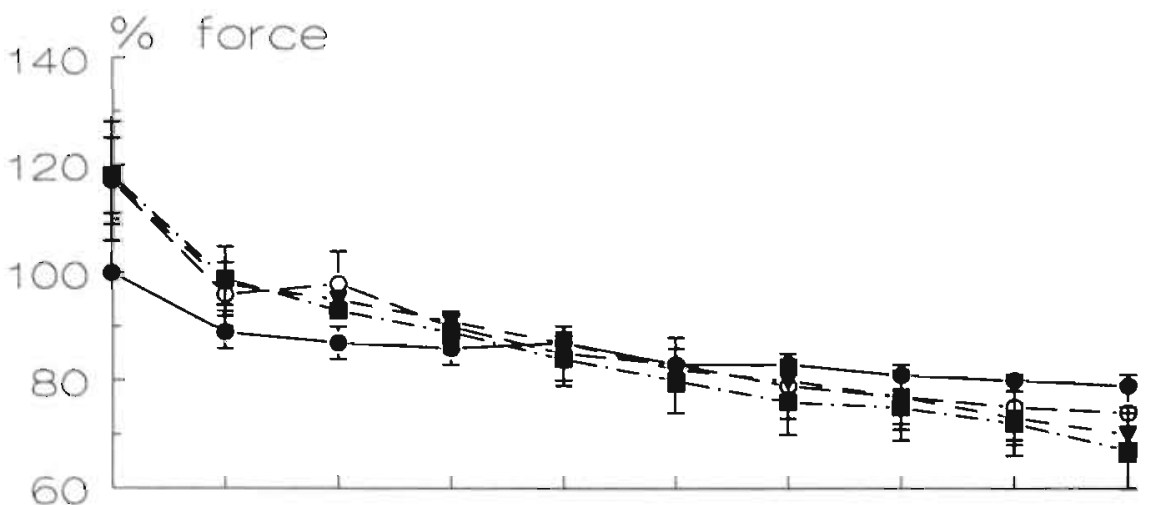

$30 \quad 60 \quad 120 \quad 180 \quad 240 \quad 300 \quad 360 \quad 420 \quad 480$ time (sec)

Figure 3.

Force of contraction during fatigue testing at 30,36,50 and 85 pulses/sec in the trained muscle. The initial mean contractile force at 30 pulses/sec is presented as $100 \%$ (values are mean \pm SE). 


\section{DISCUSSION}

Carpentier and Chachques advocate skeletal muscle stimulation at 30 pulses/sec after cardiomyoplasty and they reported significant improvement of cardiac function at this frequency, both in patients and in experimental animals $(1,11)$.

Nowadays, patients are stimulated at 30 pulses/sec, and incidentally muscle transformation has been provoked at 65 pulses/sec (12), although the effect of frequency variation has not been demonstrated.

Chronic skeletal muscle stimulation at 10 pulses/sec has shown appropriate fibre transformation (13) but contractile force appeared to be significantly lower as compared to 30 pulses/sec stimulation (2).

The present study shows that stimulation at frequencies of 5 up to 30 pulses/sec results in gradual increased contractile force, reaching about 80 $\%$ of maximal force at 30 pulses $/ \mathrm{sec}$. These findings are in line with the results of Mannion and coworkers (14) who stimulated the LD muscle in situ in dogs. Blood flow through the muscle was significantly higher at 25 pulses $/ \mathrm{sec}$ as compared to 12 pulses $/ \mathrm{sec}(0.55$ versus $0.40 \mathrm{ml} / \mathrm{min} / \mathrm{gm}$ ).

The untrained LD muscle can be classified as a fast muscle with a concomitant firing pattern of twitches from the central nervous system to the individual motor units. Extrapolation of the findings from experiments performed in rats indicates that the natural frequency is about 60 pul$\mathrm{ses} / \mathrm{sec}$ (4). Our results show that indeed near maximal force was obtained at 65 pulses $/ \mathrm{sec}$. However, when used for chronic cardiac assist, fatigue will occur in these muscles at this high frequency. In the slow (predominantly type I fibre) soleus muscle the natural frequency is about 22 pulses/sec (4). If these properties are extrapolated to the trained LD muscle, having $80 \%$ type I fibres, stimulation at about 22 pulses/sec would generate a near maximal force. Our findings are in good agreement with this assumption and show that at 20 pulses/sec already $70 \%$ of the maximal force is obtained. Besides, the fatigue test at 30 pulses/sec stimulation demonstrates significantly less strain to this trained muscle, if compared to 36 pulses/sec stimulation.

In summary, there is no effect of training of the LD muscle on the frequency range at which maximal force is being delivered. However, when the muscle is demanded to assist a failing heart by contracting up to 100 times a minute, pulse trains with a frequency of 30 pulses/sec deliver a near maximal contractile force $(80 \%)$. Muscle load at lower frequencies is significantly less in comparison to pulse trains at higher frequencies.

This indicates that LD muscle stimulation after cardiomyoplasty should preferentially be performed at 30 pulses/sec. 


\section{REFERENCES}

1. Chachques JC, Grandjean P, Schwartz K, Mihaileanu S, Fardeau M. Swynghedauw B. Fontaliran F. Romero N. Wisnewsky C. Perrier P. Chauvaud S, Bourgeois I, Carpentier A: Effect of Latissimus dorsi dynamic cardiomyoplasty on ventricular function. Circulation 1988;78(suppl III):203-216.

2. Ferguson AS, Stone HE, Roessmann U. Burke M. Tisdale E, Mortimer JT: Muscle plasticity: comparison of a 30 pulses/sec burst with 10 pulses/sec continuous stimulation. J Appl Physiol 1989;66:1 143-1151.

3. Anderson WA, Bridges CR, Chin AJ, Andersen JS, Acker MA, Hammond RL, Dimeo F. Calahan PT, Gale DR, Brown WE. Stephenson LW: Long-term neurostimulation of skeletal muscle: its potential for a tether-free biologic cardiac assist device. Pace 1988;11:2128-2134.

4. Hennig $R$, Lomo $T$ : Firing patterns of motor units in normal rats. Nature 1985:314:164-166.

5. Kochamba G, Desrosiers C, Dewar M, Chiu RC-J: The muscle-powered dualchamber counter pulsator: rheologically superior implantable cardiac assist device. Ann Thorac Surg 1988;45:620-625.

6. Havenith MG, Van der Veen FH, Glatz JFC, Lucas C, Schrijvers-van Schendel JMC, Penn OCKM, Wellens HJJ: Monitoring of muscle fibre type of canine latissimus dorsi muscle during chronic electrical stimulation by enzyme- and immunohistochemistry. In: Chiu RC-J, Bourgeois IM (Eds.). Transformed muscle for cardiac assist and repair. Futura Publishing Company Inc., Mount Kisco, NY 1990:53-61.

7. Brown MD, Cotter MA, Hudlicka O. Vrbova: The effects of different patterns of muscle activity on capillary density, mechanical properties and structure of slow and fast rabbit muscles. Pflügers Arch 1976:361:241-250.

8. Eerbeek, O, Kernell, D. Verhey BA: Effects of fast and slow patterns of tonic long-term stimulation on contractile properties of fast muscle in the cat. J Physiol 1984:352:73-90.

9. Van der Veen FH. Dassen WRM. Havenith MG, Lucas C, Van der Nagel T, Penn OCKM. Wellens HJJ: In vivo contraction analysis as a measure of canine latissimus dorsi muscle adaptation following chronic electrical stimulation. In: Chiu RC-J. Bourgeois IM (Eds.). Transformed muscle for cardiac assist and repair. Futura Publishing Company Inc., Mount Kisco, NY 1990:105-115.

10. Havenith MG. Visser R, Schrijvers-van Schendel JMC, Bosman FT: Muscle fiber typing in routinely processed skeletal muscle with monoclonal antibodies. Histochemistry 1990:93:497-499.

11. Carpentier A, Chachques JC. The use of stimulated skeletal muscle to replace diseased human heart muscle. In: Chiu RC-J (Ed.). Biochemical cardiac assist. Futura Publishing Company Inc., Mount Kisco, NY 1986;85- 102.

12. Molteñi L. Almada H, Ferreira R. Synchronously stimulated skeletal muscle graft for left ventricular assistance. J Thorac Cardiovasc Surg 1989;97:439-446.

13. Carraro H. Catani C. Saggin L: Isomyosin changes after functional electrostimulation of denervated sheep muscle. Muscle \& Nerve 1988;11:1016-1028.

14. Mannion JD. Velchik M. Hammond R, Alavi A, Mackler T, Duckett S, Staum M. Hurwitz S, Brown W, Stephenson LW: Effect of collateral blood vessel ligation and electrical conditioning on blood flow in dog latissimus dorsi muscle. J Surg Res 1989:47:332-340. 
hapter 8

\section{The importance of muscle relaxation in dynamic cardiomyoplasty}

Caroline MHB Lucas

Frederik $\mathrm{H}$ van der Veen

Emile C Cheriex

Vincent van Ommen

Olaf CKM Penn

Hein JJ Wellens

Accepted for publication in PACE 


\title{
The importance of muscle relaxation in dynamic cardiomyoplasty
}

\begin{abstract}
During the last decade, dynamic cardiomyoplasty has been introduced as a new method to treat patients with severe heart failure. This procedure consists of the wrapping of the latissimus dorsi (LD) muscle around the heart with electrical stimulation of the muscle, synchronous to cardiac contraction. The optimal pacing mode of the muscle, both during the conditioning and working period of the LD muscle, is still unclear. The pace protocol, currently used worldwide, has a maximal number of muscle tetanic contractions of 100 per minute. Data are presented on the LD muscle contraction characteristics using that protocol. Both force measurements from 6 in situ stimulated goat LD muscles and X-ray evaluation of the movement of metallic clips on wrapped LD muscles in 2 patients were used. Results demonstrate that LD muscle force is well maintained at the maximal rate of 100 contractions per minute but relaxation is severely hampered. This may lead to diminished support of the failing heart and damage of the wrapped muscle. A pacing protocol is proposed using a lower maximal stimulation rate.
\end{abstract}




\section{INTRODUCTION}

Chronic irreversible heart failure is an important cause of mortality and morbidity. Current treatment for these patients consists of drug therapy or heart transplantation and is frequently ineffective, expensive and donor hearts are scarce. Dynamic cardiomyoplasty has been introduced as a new method for permanent ventricular support in patients with heart failure (1-6).

During the cardiomyoplasty procedure a skeletal muscle, usually the left latissimus dorsi (LD) muscle, is transposed into the thoracic cavity with intact neurovascular supply. The distal, free muscle flap is then wrapped around both ventricles and fixed to the heart with multiple sutures $(4,5)$. Additional fixation of the muscle to the epicardial layer can be accomplished by using fibrin glue spray (7). Prior to the wrapping procedure two pacing wires are placed inside the LD muscle and connected with a pulse generator. Synchronization between skeletal muscle contraction and ventricular contraction is obtained through a sensing electrode on the myocardium which is connected to the pulse generator.

The main advantages of this procedure include $[8,9)$ :

1. No need for donor organs with lifelong immunosuppressive drug therapy.

2. Full implantation of the required wires and pacing device minimizing the risk of infection, which accompanies the use of external devices. 3. No risk of thromboembolic complications as in artificial support devices and therefore no need for anticoagulant therapy.

Except for a vital left LD muscle, the only mechanical devices necessary are 2 intramuscular pacing wires, a cardiac sensing lead and a specially constructed electrical pulse generator, to stimulate the skeletal muscle when wrapped around the myocardium.

Two major developments have acted as important stimuli for skeletal muscle application as a cardiac assist. Firstly, the demonstration of the feasibility to convert skeletal muscle, consisting of a mixture of type I and type II skeletal muscle fibres, into a muscle predominantly composed of slowly contracting, fatigue-resistant type I muscle fibres (10-14).

Secondly, the development of a synchronous pulse-train muscle stimulator as reported by Dewar et al (15) in 1984. Thereby, a tetanic contraction of skeletal muscle can be produced, resulting in a contraction pattern similar to ventricular systole.

Since the first clinical application of the dynamic cardiomyoplasty procedure in 1985 (16), a pulse-train generator has been used to stimulate the wrapped LD muscle in more than 100 patients (4). With this pacemaker the LD muscle is conditioned to contract during 24 hours a day, using a progressive stimulation protocol, thus enabling a continuous support for the heart.

Currently the stimulation protocol of the wrapped LD muscle is programmed as described by Chachques (5).

Although assessment of hemodynamic function has been reported in some of the patients, using this stimulation protocol, quantitative improvement of 


\section{4}

ventricular support has been described only incidentally $(4,6,17)$. Inability to demonstrate long term improvement of cardiac function in clinical and experimental studies could be related to unwanted changes in properties of skeletal muscle during chronic electrical stimulation.

We will present experimental findings supporting this hypothesis, underlining the necessity to evaluate a different stimulation protocol for the longterm use of the trained LD muscle.

\section{MATERIALS AND METHODS}

\section{Animal experiments}

All experiments were performed in accordance with the 'Guide for the Care and Use of Laboratory Animals' (18).

In 6 anesthetized goats. 2 intramuscular electrodes were positioned into the proximal part of the left LD muscle, perpendicular to the neurovascular bundle and connected to an Itrel pulse generator (19). The muscle was stimulated 24 hours a day during 12 weeks, using a progressively increasing number of pulses per minute with an individual pulse width of $210 \mu$ seconds (Table 1).

TABLE 1.

Conditioning protocol for in situ stimulation of the left latissimus dorsi muscle $(n=6)$.

\begin{tabular}{llll}
\hline week & pulses/burst & $\begin{array}{l}\text { interpulse interval } \\
\text { (msec) }\end{array}$ & bursts/min \\
\hline $1-2$ & 1 & & 50 \\
$3-4$ & 2 & 100 & 50 \\
$5-6$ & 3 & 67 & 50 \\
$7-8$ & 6 & 33 & 50 \\
$9-10$ & 6 & 33 & 60 \\
$11-12$ & 6 & 33 & 80 \\
\hline
\end{tabular}

After this conditioning period, fibre transformation was evaluated by taking biopsies from the nonstimulated right and the stimulated left LD muscle (19). Eight $\mu \mathrm{m}$ cryostat sections were stained using RllDlo (Centocor Europe), which is a mouse monoclonal antibody against the myosin heavy chain from the type I fibre (20).

After the conditioning period, the LD muscle isometric force was assessed in vivo with a force transducer, as has been described before by our group (19). Therefore, all goats were anesthetized with intravenous pentobarbital (15 mg/kg) and artificially respirated with $\mathrm{O}_{2}$ and $\mathrm{N}_{2} \mathrm{O}(1: 2)$ and $1.5 \%$ Fluothane. Two questions in particular were addressed during these measurements. 1. Are force characteristics affected when the number of bursts per minute is progressively increased from $50 / \mathrm{min}$ to $100 / \mathrm{min}$ (burst 
frequency $30 \mathrm{~Hz}$, burst duration $185 \mathrm{msec}$ or $240 \mathrm{msec}$ ). 2. Can a conditioned, transformed muscle be stimulated at a high workload ( 100 bursts per minute, burst duration $185 \mathrm{msec}$, burst frequency $30 \mathrm{~Hz}$ ) during 8 minutes without signs of fatigue?

All measurements were written on a multichannel Schwarzer (frequency response $300 \mathrm{~Hz}$ measured for $\pm 3 \mathrm{~dB}$ points) at a paper speed of $25 \mathrm{~mm} / \mathrm{sec}$.

\section{Patient evaluation}

To assess contractile properties of the $\mathrm{LD}$ muscle after a dynamic cardiomyoplasty procedure during follow-up in patients, a new method was developed (21). At the time of surgery radiopaque markers (Auto SutureTitanium, United States Surgical Corp., Norwalk, CT) were placed along the longitudinal axis of the wrapped LD muscle. Analysis of the movement of the clips during LD muscle contraction was done using biplane X-ray images recorded on videotape. Measurements were made on at least $3 \mathrm{LD}$ muscle contractions. Skeletal muscle shortening was calculated both between the stimulation electrodes and the pairs of clips, sutured on the proximal, middle and distal part of the LD muscle.

Informed consent was obtained from two patients who were analyzed after a dynamic cardiomyoplasty procedure (5), following a LD muscle conditioning period of at least 6 months. Movement of the clips was measured during stimulation of the muscle using a burst of pulses (pulse width $210 \mu \mathrm{sec}$ ) with a duration of $185 \mathrm{~ms}$, a burst frequency of $30 \mathrm{~Hz}$ and a voltage of $5 \mathrm{~V}$. Synchronization of heart to LD muscle contraction was programmed both in a 2:1 and a 1:1 mode with an upper rate of $100 \mathrm{LD}$ muscle contractions per minute.

\section{RESUTLTS}

\section{Animal data}

After 12 weeks of continuous electrical stimulation, nearly all fibres showed reactivity with the monoclonal antibody RIIDIO specific to type I myosinheavy chain ( $98 \pm 3 \%$; $\mathrm{X} \pm \mathrm{SD}$ as compared to $19 \pm 4 \%$ in the nonstimulated, contralateral LD muscle).

The force measurements, performed after the period of conditioning, demonstrated a detrimental effect on skeletal muscle function when the number of bursts was progressively increased from 50 bursts to 100 bursts per minute, both using a burst duration of 185 and $240 \mathrm{msec}$ (figures 1 and 2). Skeletal muscle force was well maintained during this increase in number of bursts. However, because the interval between two bursts did diminish progressively, it resulted in insufficient relaxation of the muscle. Especially a burst duration of $240 \mathrm{msec}$ appeared to hamper relaxation.

Figure 3 shows the results of conditioned LD muscle stimulation during the fatigue test of 8 minutes, using 100 muscle contractions/min of $185 \mathrm{msec}$ duration. Although skeletal muscle force is well preserved during this 


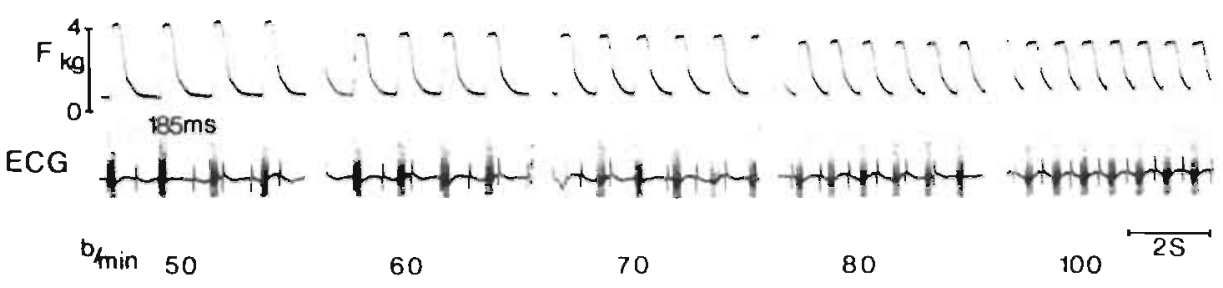

FIGURE 1 .

Isometric force registrations (F) of in situ stimulated conditioned goat $L D$ muscle. Burst duration $185 \mathrm{msec}$, burst frequency $30 \mathrm{~Hz}$. As shown an increasing number of pacing bursts per minute is given. Pacing spikes are visualized in the ECG - registration asynchronous to the R-wave because in situ stimulation of the $L D$ muscle is performed. As demonstrated increasing the number of bursts per minute results in insufficient relaxation of the LD muscle.

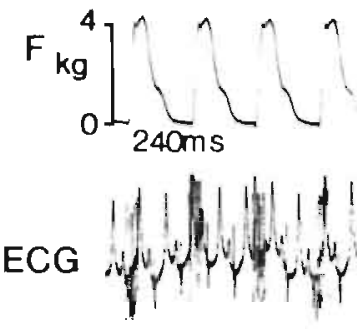

$\mathrm{b} / \mathrm{min}$
50

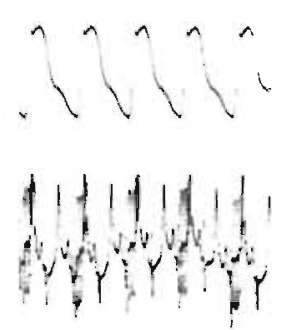

60
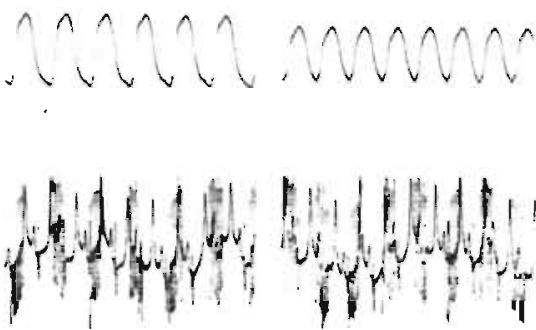

80

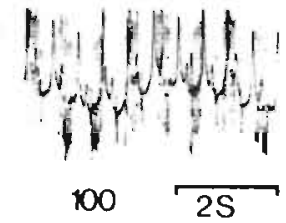

FIGURE 2.

Similar registration as figure 1 using a burst duration of $240 \mathrm{msec}$.

period, due to transformation of the muscle to a fatigue resistant muscle, a pronounced decrease in contraction speed $(\mathrm{dF} / \mathrm{dt}+)$ and particularly in relaxation speed ( $\mathrm{dF} / \mathrm{dt}-)$ is found.

\section{Patient data}

One patient, who underwent cardiomyoplasty one year earlier, showed LD muscle shortening during electrical stimulation of $23 \%$ when LD stimulation was synchronized to every other heart beat (Figure 4). The heart rate was approximately 100 beats/min. thus the LD muscle contracted 50 times per minute. When the synchronization ratio was programmed to $1: 1(100$ 

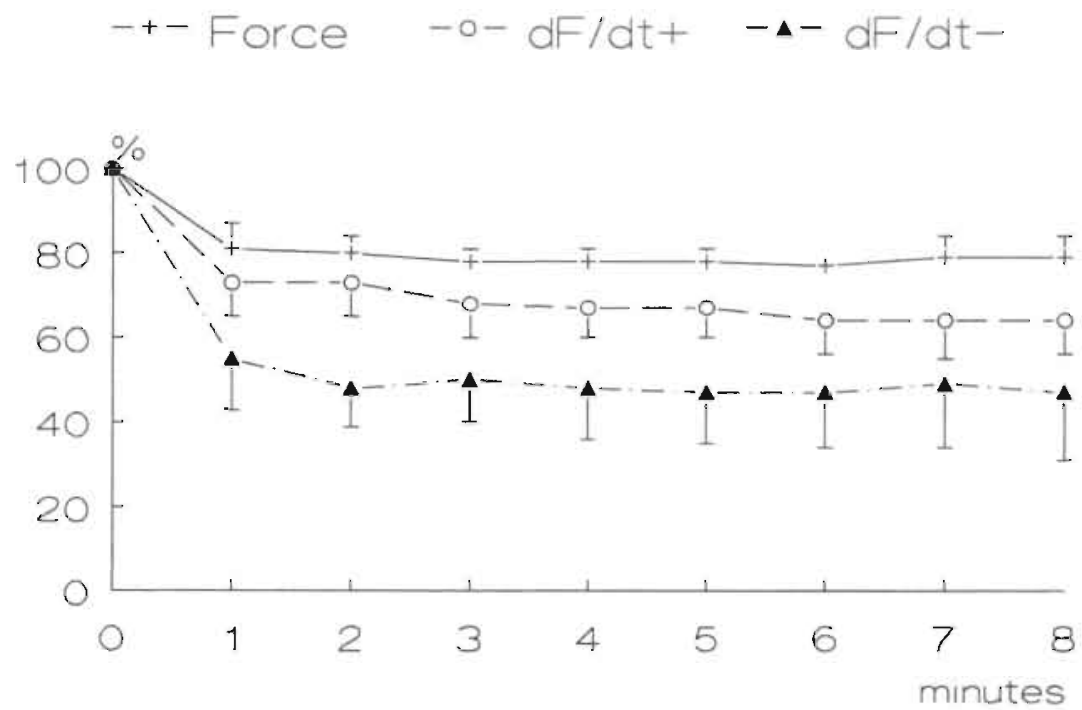

FIGURE 3.

Change in Force, contraction speed ( $d F / d t+)$ and relaxation speed (dF/dt-) during an 8 minutes fatigue test (burst duration $=185 \mathrm{~ms}$, burst frequency $=30 \mathrm{~Hz} .100 \mathrm{bursts} / \mathrm{min}$ ) as obtained during in situ isometric force measurements, using conditioned goat LD muscle ( $n=6, X \pm S D$ ).

\section{L.D. Shortening}

- 5 volt $1: 1 \quad--5$ volt $2: 1$

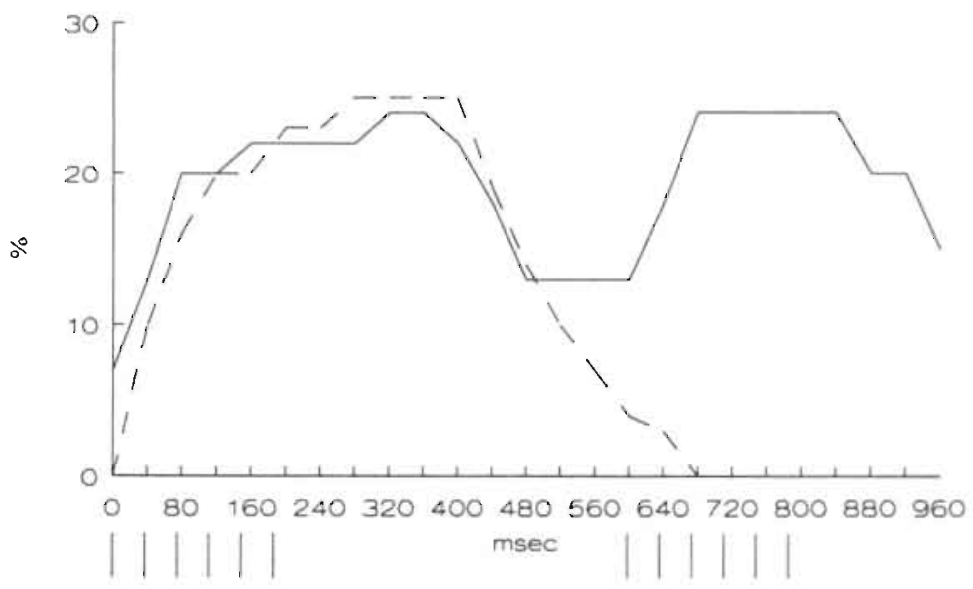

FIGURE 4.

LD muscle shortening as measured in a patient using metallic clips on the muscle. Shown are the contraction cycles during both $1: 2$ and $1: 1 \mathrm{LD}$ muscle contraction - cardiac contraction synchronization. The heart rate of the pattent was approximately 100 beats $/ \mathrm{min}$. Below the time scale the individual spikes of the pulse generator are visualized. 


\section{8}

LD muscle contractions per minute). LD shortening diminished to $11 \%$ with the muscle appearing to remain in a contractile state. Reconstruction of the contraction cycle of the LD muscle showed a contraction period of $180 \mathrm{msec}$, a tetanic phase of $200 \mathrm{msec}$ and a relaxation period of $280 \mathrm{msec}$. Therefore, one skeletal muscle contraction cycle had a total duration of about 680 msec. The same phenomenon was observed in the second patient who was studied two years after the cardiomyoplasty procedure. Apparently, above a certain stimulation rate, the slowly contracting latissimus dorsi muscle is not only still in contracture during diastole, but even at the start of the next systole.

\section{DISCUSSION}

Skeletal muscle shows important changes following continuous electrical stimulation $(10,13,19)$. It has been demonstrated that, after a certain stimulation period, almost complete transformation occurs from predominantly fast-twitch type II fibres to slow-twitch type I fibres. This is accompanied by an increase in number of capillaries and mitochondrial volume $(22,23)$, an increase in oxidative enzymes (24) and a decrease in glycolvtic enzymes. Moreover, a decrease in sarcoplasmic volume with a decrease in $\mathrm{Ca}^{2+}$-transport ATPase and $\mathrm{Ca}^{2+}$-binding proteins has been shown (22). These changes result in an improved fatigue-resistancy of the conditioned muscle with significant preservation of force during continuous contraction of this muscle $(13,25)$. We found that this is seen even when 100 bursts of $185 \mathrm{msec}$ are applied to the LD muscle in one minute.

However, the transformation of skeletal muscle after long-term stimulation has negative effects on skeletal muscle function, which are of importance when used for cardiac assist $(26,27)$. The marked decrease in speed of contraction and relaxation which is related to the decrease in sarcoplasmic volume, the decrease in $\mathrm{Ca}^{2+}$-transporting enzymes and the slower rate of crossbridge cycling in the type I muscle fibres, compared to the fast-twitch type II fibres, may not be beneficial for this long-term application of the LD muscle. Our results demonstrate that the increased slowness of the conditioned LD is hampering LD muscle function, in particular when a high number of burst contractions/min is used. We found that the programming of a high number of bursts $(100 / \mathrm{min})$, as is routinely used in patients after cardiomyoplasty, resulted in a prolonged contraction and insufficient relaxation speed of the wrapped muscle. Because LD skeletal muscle blood flow is reduced during contraction of the muscle $(8,28)$, adequate length of the relaxation phase is crucial. These periods of insufficient blood flow could have unwanted long-term effects on skeletal muscle function as described by Gorman et al, who found severe skeletal muscle 'stunning' after ischemia (29). Furthermore diastolic filling of the heart may be obstructed by the prolonged contractile state of the wrapped muscle. Also insufficient relaxation of the latissimus dorsi muscle may impair coronary blood flow leading to myocardial ischemia. These phenomena could have been responsible for 
the diffuse subepicardial microscopic foci of myocardial cell necrosis which Rossi et al found in a patient who died 4 months after a dynamic cardiomyoplasty procedure (30).

A negative contractile effect during high rate pacing has also been noted by Anderson et al (31), using the LD muscle in a skeletal muscle ventricle. In this model the muscle is used for diastolic counterpulsation. A significant decrease in the diastolic augmentation, as produced by this skeletal muscle ventricle, was observed when the synchronization ratio was switched after 12 weeks of stimulation from a $1: 2$ to a $1: 1$ synchronization ratio. Resetting the pacemaker again to a $1: 2$ synchronization ratio did not restore the original augmentation completely.

Using a mathematical model to evaluate the optimal timing of LD muscle contraction during cardiac systole, Levin et al (32) also speculated about the detrimental effect of approximately 100 pacing bursts/min of $185 \mathrm{~ms}$ on cardiac filling, even with an unconditioned fast contracting LD muscle.

In conclusion, we recommend not to pace the wrapped LD muscle in a $1: 1$ synchronization mode to avoid rates of 100 skeletal muscle contractions per minute. Both the optimal burst duration and the number of bursts/min have to be evaluated individually in relation to the decrease in contraction and relaxation speed after the conditioning period of the wrapped LD muscle. Our proposal for a new conditioning protocol for the LD muscle and the mode of stimulation after full transformation of this wrapped muscle is shown in Table 2 (see also chapter 9). It shows a marked reduction in burst duration and the maximal number of bursts/min, compared to the one currently in clinical use.

TABLE 2.

New stimulation protocol for the wrapped latissimus dorsi muscle after a cardiomyoplasty procedure.

\begin{tabular}{llcc}
$\begin{array}{l}\text { weeks after } \\
\text { operation }\end{array}$ & pulses/burst & $\begin{array}{l}\text { interpulse interval } \\
\text { (msec) }\end{array}$ & bursts/min \\
\hline $1-2$ & 0 & & 0 \\
$3-4$ & 2 & 100 & 50 \\
$5-6$ & 3 & 50 & 50 \\
$7-8$ & 4 & 30 & 50 \\
$9-10$ & 5 & 23 & 50 \\
$11-$ & 6 & 20 & 60 \\
\hline
\end{tabular}




\section{REFERENCES}

1. Moreira LPG, Stolf NAG, Jatene AD: Latissimus dorsi cardiomyoplasty in the treatment of patients with dilated cardiomyopathy. Circulation 1990;82(suppl IV): 257-263.

2. Magovem GJ, Heckler F, Park SB, Christlieb IY, Burkholder J, Maher T, Benckart D. Magovern Jr GJ, Kao R: Paced skeletal muscle for dynamic cardiomyoplasty. Ann Thorac Surg 1988;45:614-619.

3. Molteni L, Almada H, Ferreira R: Synchronously stimulated skeletal muscle graft for left ventricular assistance. J Thorac Cardiovasc Surg 1989;97:439-443.

4. Grandjean PA. Austin L, Chan S. Bourgeois IM: Dynamic cardiomyoplasty: Clinical follow-up results. J Cardiac Surg 1991;6(suppl):80-88.

5. Chachques JC, Grandjean P, Schwartz K, Mihaileanu S, Fardeau M, Swynghedauw B, Fontaliran F, Romero N, Wisnewsky C, Perier P, Chauvaud S, Bourgeols I. Carpentier A: Effect of Latissimus dorsi dynamic cardiomyoplasty on ventricular function. Circulation 1988;78(suppl III);203-216.

6. LFP Moreira, NAG Stolf, AD Jatene: Benefits of cardiomyoplasty for dilated cardiomyopathy. Sem Thorac Cardiovasc Surg 1991;3(2);140-144.

7. Lorusso R. Lucas C, Van der Veen F, Habets J, van der Nagel T, Havenith H, Wellens HJ, Penn OC: Fibrin sealant application during dynamic cardiomyoplasty. In: DAllessandro LC (Ed.): Heart Surgery 1991. Casa Editrice Scientifica Internationale. 1991:579-584.

8. Geddes LA, Badylak SF, Wessale JL. Janes W, Bourland JD. Tacker WA, Stevens L: The use of electrically stimulated skeletal muscle to pump blood. PACE 1990:13:344-362.

9. Mannion JD. Stephenson LW: Potential uses of skeletal muscle for myocardial assistance. Surg Clin North Am 1985;65:679-687.

10. Salmons S, Henriksson J: The adaptive response of skeletal muscle to increased use. Muscle \& Nerve 1981:4:94-105.

11. Pette D: Activity-induced fast to slow transition in mammalian muscle. Med Sci Sports Exc 1984:16:517-528.

12. Ianuzzo CD. Hamilton N, O'Brien PJ, Desrosiers C, Chiu RC-J: Biochemical transformation of canine skeletal muscle for use in cardiac assist. J Appl Physiol 1990;68(4): $1481-1485$.

13. Clark BJ, Acker MA, McCully K, Subramanian HV, Hammond RL, Salmons S, Chance B, Stephenson LW: In vivo $31 \mathrm{P}-\mathrm{NMR}$ spectroscopy of chronically stimulated canine skeletal muscle. Am J Physiol 1988;254:C258-266.

14. Acker MA. Hammond RL, Mannion JD, Salmons S, Stephenson LW: An autologous biologic pump motor. J Thorac Cardiovasc Surg 1986:92:733-746.

15. Dewar ML. Drinkwater DC, Wittnich C. Chiu RC-J: Synchronously stimulated skeletal muscle graft for myocardial repalr. J Thorac Cardiovasc Surg 1984; 87:325-331.

16. Carpentier A. Chachques JC: Myocardial substitution with a stimulated skeletal muscle: First successful clinical case. Lancet 1985;1:1267-1270.

17. Molteni L. Almada HE, de Palma C, Ferreira RF: Dynamic cardiomyoplasty: Clinical experience. In: Chiu RC-J. Bourgeois IM (Eds.): Transformed muscle for cardiac assist and repair. Futura Publishing Company Inc., Mount Kisco, NY 1990; 197-198.

18. National Academy of Sciences: Guide for the Care and Use of Laboratory Animals. DHHS publication No. NIH 85-23, revised 1985. 
19. Lucas CM, Havenith MG, Van der Veen FH, Habets J, van der Nagel $T$, Schrijvers-van Schendel JM, Penn OC. Wellens HJ: Changes in canine latissimus dorsi muscle during 24 weeks of continuous electrical stimulation. J Appl Physiol 1992;72:828-835.

20. Havenith MG, Visser R, Schrijvers-van Schendel JM, Bosman FT: Muscle fibre typing in routinely processed skeletal muscle with monoclonal antibodies. Histochemistry 1990;93:497-499.

21. Van der Veen FH, Lucas C, Lorusso R, van der Nagel T, Penn OCKM, Wellens HJJ: A new method to select stimulus strength after cardiomyoplasty. J Cardiac Surgery 1991;6(suppl):259-264.

22. Reichmann H, Hoppeler H. Mathieu-Costello O, von Bergen F, Pette D: Biochemical and ultrastructural changes of skeletal muscle mitochondria after chronic electrical stimulation in rabbits. Pflügers Arch 1985;404:1-9.

23. Eisenberg BR, Salmons S: The reorganization of subcellular structure in muscle undergoing fast-to-slow type transformation. Cell Tiss Res 1984;220:22 1-230.

24. Henriksson J, Chi M-Y, Hintz CS, Young DA, Kaiser KK, Salmons S, Lowry OH: Chronic stimulation of mammalian muscle: Changes in enzymes of six metabolic pathways. Am J Physiol 1986;251:C614-C632.

25. Leirner AA, Moreira LFP, Chagas ACP. Cestari LA, Oshiro MS, Nakayama E, da Luz PL, Stolf AG, Jatene AD: Biomechanical circulatory assistance: Importance of aerobic capacity of normal and conditioned skeletal muscle. Trans Am Soc Artif Int Organs 1988;34:716-720.

26. Salmons S, Jarvis JC: The working capacity of skeletal muscle transformed for use in a cardiac assist role. In: Chiu RC-J, Bourgeois IM (Eds.). Transformed muscle for cardiac assist and repair. Futura Publishing Company Inc., Mount Kisco, NY 1990:89-104.

27. Salmons S, Jarvis JC: Cardiomyoplasty: A look at the fundamentals. In: Carpentier A, Chachques JC, Grandjean P [Eds.]. Cardiomyoplasty. Futura Publishing Company, Inc., Mount Kisco, NY 1991:3-17.

28. Tacker WA, Geddes LA, Janas W, Babbs JF, Badylak SF: Comparison of canine skeletal muscle power from twitches and tetanic contractions in untrained muscle: A preliminary report. J Cardiac Surg 1991:6(suppl):245-251 .

29. Gorman MW, Barclay JK, Sparks HV: Effects of ischemia on $\mathrm{VO}_{2}$, tension, and vascular resistance in contractıng canine skeletal muscle. J Appl Physiol 1988; 65(3); 1075-1081.

30. Rossi MA, Braile DM, Souza DRS, Santoz JLV, Thevenard RS, Pinto GH, Suzigan S, Grecco O: Dynamic cardiomyoplasty in chronic Chagas' heart disease: Clinicopathological data. Ann Thorac Surg 1991:51:649-651.

31. Anderson DR, Pochettino A. Hammond RL, Hohenhaus E, Spanta AD, Bridges CR. Lavine S, Bhan RD, Colson M, Stephenson LW: Autogenously lined skeletal muscle ventricles in circulation: $U p$ to nine months experience. J Thorac Cardiovase Surg 1991;101:661-670.

32. Levin HR, Tsitlik JE, Halperin HR: Optimization of the timing of skeletal to cardiac muscle contraction during dynamic cardiomyoplasty: Analysis using a mathematical model. J Cardiac Surg 1991;6(suppl):236-244. 
hapter 9

\section{A new stimulation protocol for cardiac assist using the latissimus dorsi muscle}

Caroline MHB Lucas

Marie-Louise Dubelaar

Frederik $H$ van der Veen

Elisa N Kloosterman - Castro-Ravelo

Miek G Havenith

Jo Habets

Theo van der Nagel

Hein JJ Wellens

Presented at the North American Society of Pacing and Electrophysiology, PACE 1992:15;561.

Submitted for publication. 


\title{
A new stimulation protocol for cardiac assist using the latissimus dorsi muscle
}

\begin{abstract}
When treating severe cardiac failure with dynamic cardiomyoplasty, knowledge about the optimal way of stimulating the latissimus dorsi (LD) muscle is of obvious importance. We evaluated a new stimulation protocol in 4 goats, using in situ electrical stimulation of the left LD muscle. Stimulation was started using a burst of 2 pulses with an interpulse interval of $100 \mathrm{~m}$ sec for 50 bursts $/ \mathrm{min}$. The number of pulses was increased every 2 weeks concomitant with a decrease in interpulse interval. This resulted after 12 weeks in 60 bursts/min, using bursts of 6 pulses with an interpulse interval of $20 \mathrm{msec}$. Force measurements. which were done every 2 weeks, showed an early decrease in contraction- and relaxation speed, as reflected in the ripple (= interstimulus amplitude/peak force amplitude measured at $10 \mathrm{~Hz}$ ). Fatigue-resistance increased significantly within 4 weeks of conditioning, as indicated by preservation of force, positive $\mathrm{dF} / \mathrm{dt}$ and negative $\mathrm{dF} / \mathrm{dt}$. Full preservation of these variables was seen even during a 1 hour fatigue test at the end of the conditioning period. Skeletal muscle enzyme-activity. as indicator of muscle damage, showed a significant, rise in creatine kinase enzyme-activity, only on the first day following the start of LD stimulation. LD muscle biopsies revealed almost complete transformation to type I muscle fibres with a significant increase in capillaryto-fibre ratio when compared to the non-stimulated LD muscle. Some biopsies, in particular near the electrodes however, did show some signs of skeletal muscle damage. Contraction characteristics of the fully transformed LD muscles were tested by increasing the number of bursts of 6 pulses from $50 / \mathrm{min}$ to $100 / \mathrm{min}$. Interpulse intervals of 20 and 33 msec were used. These tests revealed that maximal force, positive $\mathrm{dF} / \mathrm{dt}$ and negative $\mathrm{dF} / \mathrm{dt}$ was reached with 50 bursts/min using a 6 pulse burst with interpulse intervals of $20 \mathrm{msec}$.
\end{abstract}




\section{INTRODUCTION}

In the last decade, an increasing number of centers are evaluating dynamic cardiomyoplasty as a new therapy to treat patients with severe heart failure (1-3). During this procedure the latissimus dorsi (LD) muscle is wrapped around the heart and electrically stimulated, synchronously with the heart beat. It has been shown that the LD muscle can be conditioned to contract continuously during 24 hours a day $(4,5)$. An important question is the optimal way of stimulating the skeletal muscle, both during and after the conditioning period. In this article we present an alternative conditioning protocol of the LD muscle and the application of this protocol in fully trained LD muscles. Contraction characteristics of these transformed muscles will be compared with results obtained with the currently applied clinical protocol (6). Goats were used in whom the left LD muscle was paced in situ. The effects of electrical stimulation were evaluated by performing in situ force measurements, measuring the release of skeletal muscle enzymes during the conditioning period and by examining LD muscle biopsies.

\section{MATERIALS AND METHODS}

Four goats were used with body weights ranging from 41 to $66 \mathrm{~kg}$. All experiments were performed in accordance with the 'Guide for the Care and Use of Laboratory Animals' (7).

Preparation of the goats

The animals were anesthetized initially with sodium thlopental (Nesdonal 15 $\mathrm{mg} / \mathrm{kg}$ body weight) into the jugular vein, intubated and ventilated with oxygen/nitrous oxide $(1: 2)$ and $1.5 \%$ Fluothane. Under sterile conditions and pre-operative antibiotic cover (Amfipen $1000 \mathrm{mg}$ iv), a $15 \mathrm{~cm}$ longitudinal skin incision was made in the left midaxillary line to expose the pedicle of the LD muscle. Two intramuscular electrodes (Medtronic SP5528) were inserted into the proximal part of the left latissimus dorsi muscle, perpendicular to the neurovascular pedicle, and connected to an Itrel pulse generator (Medtronic SP7420). The pulse generator was positioned in a subcutaneous pocket in the interscapular region. All goats received buprenorfine (Temgesic $0.6 \mathrm{mg} \mathrm{im}$ ) for two days for analgesia and one dosage of Amfipen $1000 \mathrm{mg}$ im as antibiotic treatment.

\section{LD muscle conditioning}

Stimulation of the LD muscle was started two weeks after operation with a short burst consisting of 2 pulses with an individual pulse width of 210 $\mu$ seconds. Interpulse interval was programmed at $100 \mathrm{msec}$. Every two weeks the number of pulses in a burst was increased concomitant with a decrease in interpulse intervals. This resulted in a stimulation pattern 10 weeks after operation using 60 bursts per minute consisting of 6 pulses with interpulse intervals of $20 \mathrm{msec}$ (Table 1). At the start of the LD muscle 
146

TABle 1.

Conditioning protocol of the left LD muscle.

\begin{tabular}{llcc}
$\begin{array}{l}\text { weeks after } \\
\text { operation }\end{array}$ & $\begin{array}{l}\text { number of } \\
\text { pulses/burst }\end{array}$ & $\begin{array}{l}\text { interpulse interval } \\
\text { (msec) }\end{array}$ & $\begin{array}{l}\text { number of } \\
\text { bursts/min }\end{array}$ \\
\hline $1-2$ & 0 & & 0 \\
$3-4$ & 2 & 100 & 50 \\
$5-6$ & 3 & 50 & 50 \\
$7-8$ & 4 & 30 & 50 \\
$9-10$ & 5 & 23 & 50 \\
$11-12$ & 6 & 20 & 60 \\
\hline
\end{tabular}

conditioning period, a voltage of $4 \mathrm{~V}$ was selected, producing a visible and palpable contraction of the muscle without causing apparent discomfort to the animal. This voltage was kept constant during the entire stimulation period.

In vivo force measurements of the $L D$ muscle

Measurements of contractile force of the left LD muscle was performed at 0 . $2,4,6,8$, and 10 weeks of electrical stimulation.

For these measurements all goats were anesthetized with thiopental (Nesdonal; $15 \mathrm{mg} / \mathrm{kg}$ iv], intubated and artificially ventilated with oxygen $/ \mathrm{ni}^{-}$ trous oxide and $1.5 \%$ Fluothane. Subsequently, they were rigidly fixed with straps on their right side on the operation table to avoid movements during contraction of the left $L D$ muscle. The left forefoot was connected to a force displacement transducer (Grass FT 10, Grass Instruments, Quincy. Mass. USA), with the left paw and left LD muscle in a position in line with the transducer, to measure the force of the stimulated skeletal muscle. Isometric twitch contractions were recorded at varying length of the LD muscle to obtain the optimal length which yielded maximal force to use for further testing. Subsequently, the ltrel pacemaker was programmed with the following parameters: Individual pulse width of $210 \mu$ seconds, a burst duration of 1 second and an interburst interval of 10 seconds. The same voltage, as programmed for conditioning of the LD muscle, was used for these measurements. Interpulse intervals from 500 to $4 \mathrm{msec}$ were used. Following these measurements a fatigue test of 8 minutes duration was performed. A burst of 6 pulses was programmed with interpulse intervals of $20 \mathrm{msec}$. Concomitantly the number of bursts per minute was programmed at 60 per minute. Voltage and pulse width were not changed. At the final force measurements, 12 weeks after operation, fatigue-resistance of the LD muscle was tested by extending the time period of this test to approximately 1 hour. Also, the effect of an increase in number of bursts from 50 to maximally 100 bursts per minute with these bursts of 6 pulses (interpulse intervals $20 \mathrm{msec}$ ) was evaluated and subsequently compared with an increase in number of bursts from $50 / \mathrm{min}$ to $100 / \mathrm{min}$, using a burst of 6 
pulses with interpulse intervals of $33 \mathrm{msec}$. This was done by measuring a total of 20 contractions of every burst protocol. Force, positive $\mathrm{dF} / \mathrm{dt}$ and negative $\mathrm{dF} / \mathrm{dt}$ of the LD muscle were recorded on a multichannel Schwarzer recorder (frequency response $300 \mathrm{~Hz}$ ) at a paper speed of $25 \mathrm{~mm} / \mathrm{sec}$ and $200 \mathrm{~mm} / \mathrm{sec}$.

Evaluation of plasma skeletal muscle enzyme levels

Four cc blood samples were taken from the anesthetized goats before and immediately after every force measurement. Twentyfour hours after these tests another sample was obtained from the jugular vein. All samples were immediately centrifuged for 10 minutes at $1.500 \mathrm{~g}$. The supernatant plasma was collected and stored at $-50^{\circ} \mathrm{C}$ for analysis of plasma activity of creatine kinase (CK), aspartate aminotransferase (AST) and lactate dehydrogenase (LDH). Activities of CK, AST and LDH were determined spectrophotometrically using commercially available test kits (NAC-activated CK test from Merck Diagnostica, LDH and AST test from Boehringer) and a centrifugal analyzer (Cobas Bio System, Hoffman-La Roche). Activities were determined in duplicate at $25^{\circ} \mathrm{C}$ and expressed in micromoles of substrate converted per minute (U).

\section{LD muscle biopsies}

Following the final force measurements 12 weeks after the start of the study, biopsies were taken from left and right LD muscles. Both muscles were exposed completely to get an optimal vew. From each side a total of 3 biopsies were taken starting at the pedicle (biopsy A), then one from the middle (biopsy B) and lastly one from the distal part (biopsy C) of both LD muscles. Also specimen were obtained from tissue surrounding both intramuscular electrodes. All biopsies were stained with hematoxylin eosin and elastin-von Giesson. Muscle fibre-typing was performed, as described before, using R11D10, a monoclonal antibody aimed at myosin heavy chain from type I muscle fibres (5), combined with alkaline phosphatase staining to demonstrate the capillaries (8).

\section{Statistical analysis}

For all force and enzyme measurements repeated-measures one way analysis of variance was used followed by a Student's $t$ test for unpaired samples. Differences were accepted to be significant at $P<0.05$. Values are presented as mean $\pm \mathrm{SD}$.

\section{RESULTS}

All goats tolerated the period of continuous LD contraction well without signs of discomfort.

The ripple, which is defined as the ratio between interstimulus amplitude divided by peak force amplitude measured with a 1 second burst of $10 \mathrm{~Hz}$. demonstrated a decrease which appeared significant within 4 weeks of 


\section{8}

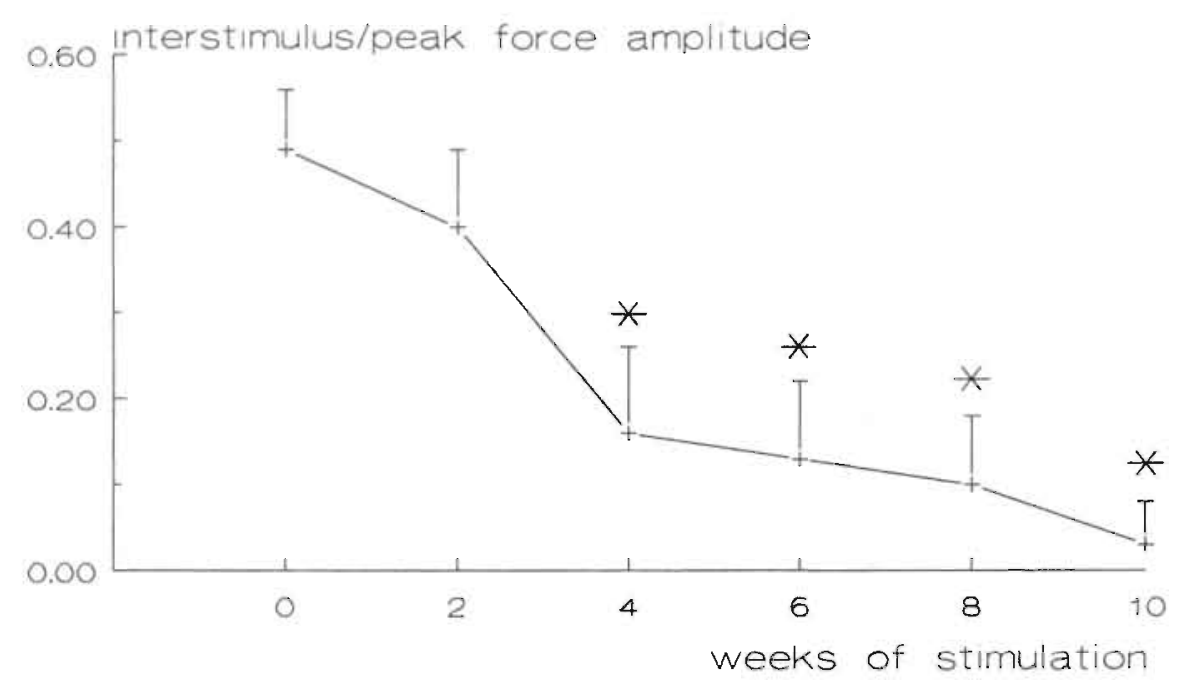

FIGURE 1 .

Change in ripple. defined as interstimulus amplitude divided by peak force amplitude, measured with a burst with an interpulse interval of $100 \mathrm{msec}$. ": significant change in ripple compared to pre-conditioning value, $P<0.05$.

stimulation. During the remaining period of LD muscle pacing a further decrease was observed finally resulting in a ratio of $0.03 \pm 0.05$ after 10 weeks of stimulation (figure 1).

This decrease in ripple enclosed both an increase in time-to-peak from 58 \pm 4 to $86 \pm 8 \mathrm{msec}(\mathrm{P}<0.001)$ and an increase in half-relaxation time from $41 \pm 7$ to $100 \pm 14 \mathrm{msec}(\mathrm{P}<0.001)$ after 10 weeks.

Force-frequency relation demonstrated a significant leftward shift after this conditioning period, resulting in the achievement of a higher maximal force at lower frequencies. This difference was significant at interpulse intervals of 100,65 and $50 \mathrm{msec}$ (figure 2).

The results of all fatigue tests are visualized in figure 3 which shows the force, positive $\mathrm{dF} / \mathrm{dt}$ and negative $\mathrm{dF} / \mathrm{dt}$ at the end of the test, relative to the values at the start. This figure demonstrates already after 2 weeks of stimulation an important increase in preservation of force and positive $\mathrm{dF} / \mathrm{dt}$ as an index of contraction speed. It took another two weeks for the negative $\mathrm{dF} / \mathrm{dt}$. as a parameter of relaxation speed, to gain significant improvement. Already after 4 weeks of stimulation, there was preservation of force, positive $\mathrm{dF} / \mathrm{dt}$ and negative $\mathrm{dF} / \mathrm{dt}$ during the test period of 8 minutes. After completion of the conditioning period of the LD muscles no important decrease in measured parameters was seen, even during the fatigue test of one hour duration. In figure 3 the bars at 10 weeks of stimulation indicate results after 1 hour of fatigue testing.

Evaluation of contraction characteristics of the fully conditioned LD muscles is shown in figure 4 . Force (figure $4 \mathrm{a}$ ), positive $\mathrm{dF} / \mathrm{dt}$ (figure $4 \mathrm{~b}$ ) and negative $\mathrm{dF} / \mathrm{dt}$ (figure $4 \mathrm{c}$ ) is displayed relative to the maximal values 

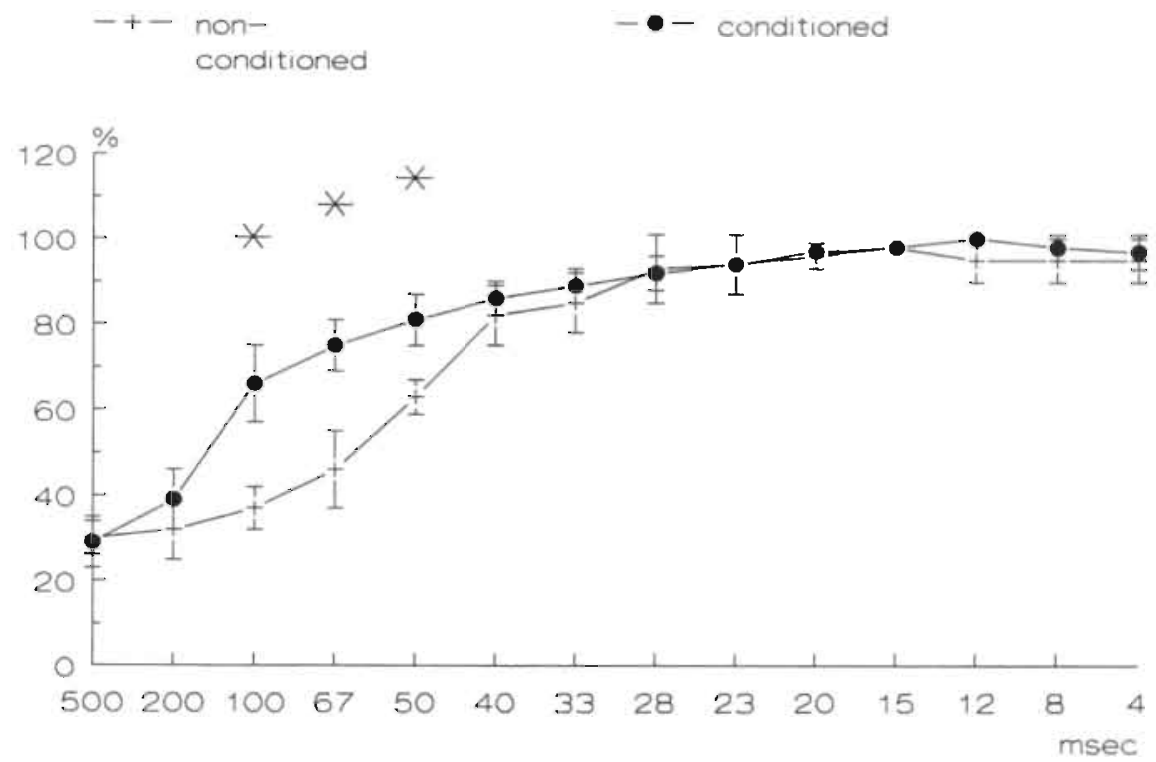

FIGURE 2.

Muscle force nonmalized to maximum tetanic tension, measured during stimulation using bursts with interpulse intervals from 500 to $4 \mathrm{msec}$ both before and after a conditioning period of 12 u'seks (": significantly different from the pre-conditioning value, $P<0.05$ ).

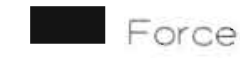

\section{प्या/I Pos. $d F / d t$ WIA Neg. $d F / d t$}

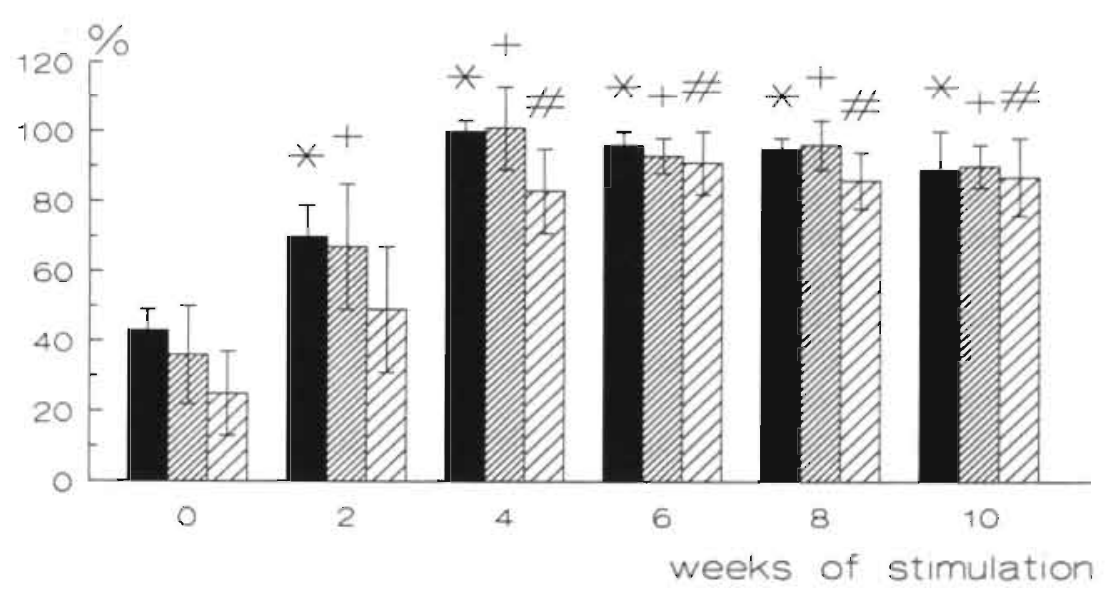

FIGURE 3

Remaining force. positive $d F / d t$ and negative $d F / d t$ after an 8 minutes fatigue test, relative to the starting values. Weeks of conditioning are indicated on the horizontal axis. At 10 weeks of stimulation. relative remaining values after 1 hour testing are demanstrated. *, +, ; $_{\text {; significant }}$ increase in relative remaining force, positive $d F / d t$ and negative $d F / d t$ respectively compared to the pre-conditioning value with $P<0.05$. 


\section{0}

TABLE 2.

Relative decrease in force, pos. $d F / d t$ and negative $d F / d t$ comparing the application of 100 bursts/min to 50 bursts/min. Results of bursts with interpulse intervals of $33 \mathrm{msec}$ and 20 msec are demonstrated $(n=4, X \pm S D)$.

\begin{tabular}{lll}
\hline & $33 \mathrm{msec}$ & $20 \mathrm{msec}$ \\
\hline Force & $22 \% \pm 8 \%$ & $19 \% \pm 7 \%$ \\
Pos. dF $/ \mathrm{dt}$ & $25 \% \pm 7 \%$ & $23 \% \pm 9 \%$ \\
Neg. dF $/ \mathrm{dt}$ & $36 \% \pm 19 \%$ & $33 \% \pm 12 \%$ \\
\hline
\end{tabular}

obtained with 50 bursts/min using bursts of 6 pulses with interpulse intervals of $33 \mathrm{msec}$. As shown, increasing the number of bursts resulted in a decrease in force, positive $\mathrm{dF} / \mathrm{dt}$ and negative $\mathrm{dF} / \mathrm{dt}$ both with bursts with 33 and with 20 msec interpulse intervals. Application of 50 and 60 bursts/ min using bursts of 6 pulses with an interpulse interval of $20 \mathrm{msec}$ lead to a significantly higher relative force compared to the burst with the interpulse interval of $33 \mathrm{msec}$. Positive $\mathrm{dF} / \mathrm{dt}$ was significantly higher using the $20 \mathrm{msec}$ interpulse interval for 50 to $100 \mathrm{bursts} / \mathrm{min}$. However, the relative decrease in force, positive $\mathrm{dF} / \mathrm{dt}$ and negative $\mathrm{dF} / \mathrm{dt}$, when comparing 100 bursts/min to 50 bursts/min, was not significantly different between bursts with 33 or $20 \mathrm{msec}$ interpulse intervals (Table 2). No significant differences in negative $\mathrm{dF} / \mathrm{dt}$ were seen when comparing interpulse intervals of $20 \mathrm{msec}$ and 33 msec (figure $4 \mathrm{c}$ ).

Plasma skeletal muscle enzyme levels

As shown in figure $5 \mathrm{CK}-$. LDH- and AST-enzyme levels appeared very stable during the whole conditioning period. Only on the day following the first force measurements and the start of the continuous electrical stimulation a significant rise was seen in CK (figure 5a), and a tendency to a rise in LDH (figure 5b). However, those values had normalized again at the start of the second force measurements after two weeks of stimulation. During the remaining conditioning period, no significant changes in enzyme levels could be detected, although $\mathrm{CK}$ demonstrated a tendency to an increase after the last force measurements, which included the 1 hour fatigue test, after 10 weeks of conditioning.

\section{LD muscle biopsies}

Fibre-typing demonstrated almost complete transformation from the fastfatiguable type II fibres to the non-fatiguable type I fibres at all locations (Table 3). Also, significant increases in capillary-to-fibre ratio were visible. All fibres had a rounded appearance after this stimulation period. In one goat signs of some LD muscle fibre damage were visible, as evidenced by the presence of atrophic fibres invaded by inflammatory cells and target fibres next to whorled fibres. Also central nuclei were present. Interfascicular fatty tissue had increased in the majority of biopsies of the stimulated LD muscles. Near the electrodes some destruction of muscle cells, nerve branches and vessels was seen. 
Force

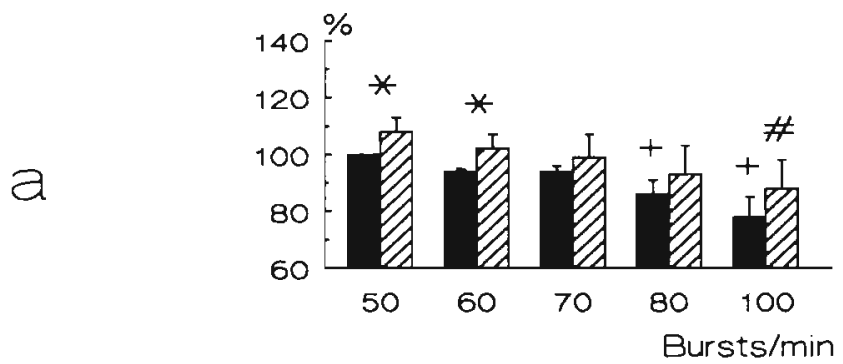

pos.dF/dt

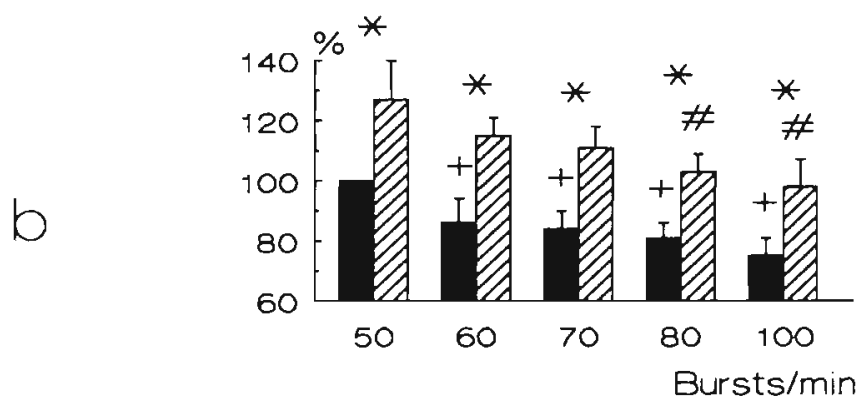

neg.dF/dt

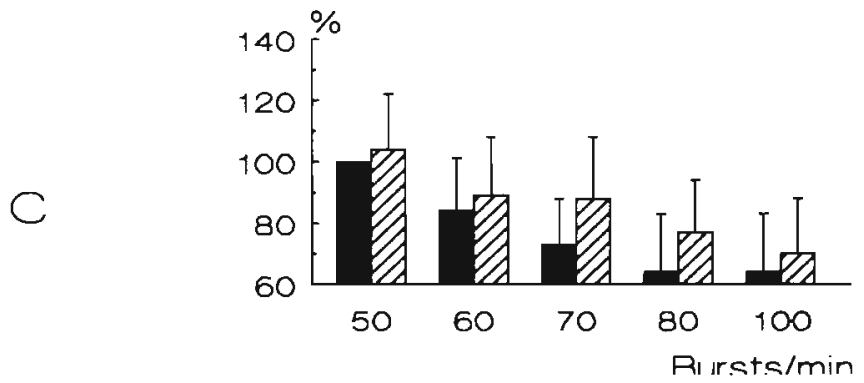

FIGURE 4

Force (a). positive $d F / d t$ (b) and negative $d F / d t$ (c) as measured using two different burst frequencies (interpulse interval $33 \mathrm{msec}=$ dark bar and $20 \mathrm{msec}=$ hatched bar) and an increasing number of bursts per minute. Values are displayed relative to the value as obtained using 50 bursts/min with interpulse' intervals of $33 \mathrm{msec}$. ": significant difference between interpulse interval of $33 \mathrm{msec}$ and $20 \mathrm{msec}$ with $P<0.05$. +: significant decrease in force and positive $d F / d t$, using an interpulse interval of $33 \mathrm{msec}$, comparing an increasing number of bursts to 50 bursts/min. \#: significant decrease in force and positive $d F / d t$ using an interpulse interval of 20 msec. comparing an increasing number of bursts to 50 bursts/min. 


\section{2}

TABLE 3.

Percentage of type I muscle fibres and capillary/fibre ratio in right and left $L D$ muscles at three different locations (A, B and C).

\begin{tabular}{lllll}
\hline Location & $\begin{array}{l}\text { right LD } \\
\text { type I (\%) }\end{array}$ & $\begin{array}{l}\text { right LD } \\
\text { cap/fibre }\end{array}$ & $\begin{array}{l}\text { left LD } \\
\text { type I (\%) }\end{array}$ & $\begin{array}{l}\text { left LD } \\
\text { cap/fibre }\end{array}$ \\
\hline A & $24 \pm 4$ & $1.12 \pm 0.10$ & $96 \pm 6^{*}$ & $1.35 \pm 0.06^{*}$ \\
B & $21 \pm 3$ & $1.05 \pm 0.12$ & $99 \pm 1^{*}$ & $1.45 \pm 0.08^{*}$ \\
C & $20 \pm 6$ & $1.07 \pm 0.06$ & $96 \pm 8^{*}$ & $1.41 \pm 0.10^{*}$ \\
\hline
\end{tabular}

Data are presented as mean \pm S.D. ${ }^{*}$; significant difference between right and left LD muscles $(p<0.05)$.

\section{DISCUSSION}

The ultimate goal of a dynamic cardiomyoplasty procedure is to obtain maximal assistance of the failing heart by the contracting wrapped LD muscle.

Optimal electrical stimulation of this LD muscle plays an important role in attaining this target. This holds true both for the conditioning period of the LD muscle and for the period thereafter when the muscle has been transformed to a muscle almost fully composed of non-fatiguable type I fibres (4, 5]. This transformation, however, not only leads to an important fatigue resistance but is also accompanied by a significant decrease in contraction and relaxation speed $(4-6,9)$. These phenomenons are unwanted side effects of the conditioning process.

First of all, it results in a contraction cycle of the transformed LD muscle which appears to be to long (10, see Chapter 8 ) to be able to allow the wrapped LD muscle sufficient relaxation time. Therefore, the currently used clinical protocol as described by Chachques et al (6) should be adapted.

Secondly, speed of contraction of the muscle could become to slow and consequently loose its power to effectively assist the heart after the wrapping procedure (11-13).

Considering these points, we wanted to test a conditioning protocol that uses a short burst with an increasing number of pulses, together with a restricted number of maximal bursts/min. As demonstrated, also this protocol resulted in comparable significant decreases in contraction and relaxation speed considering the changes in ripple (5). In particular, the increase in $1 / 2$ relaxation time of the LD muscle appeared pronounced. With this protocol important fatigue-resistance was already reached after 4 weeks. At this time only a slight decrease in negative $\mathrm{dF} / \mathrm{dt}$, as a sign of fatigue $(14,15)$, was seen after the 8 minutes test, which had disappeared after 6 weeks of stimulation. After completion of the full conditioning period of 10 weeks, no significant decrease in force, positive $\mathrm{dF} / \mathrm{dt}$ and negative $\mathrm{dF} / \mathrm{dt}$ was observed during the test of 1 hour. This is in contrast to results we obtained in conditioned goat LD muscles, using the protocol as described by Chachques et al (6). We found that application of a burst of 6 pulses with interpulse intervals of $33 \mathrm{msec}$ and a burst duration of $185 \mathrm{msec}$ during 100 
bursts/min resulted in significant decreases in positive and negative $\mathrm{dF} / \mathrm{dt}$, despite good preservation of force during the 8 minute test (see Chapter 3 ). Therefore, in our opinion, it is important to evaluate not only the preservation of force after a conditioning period of the $\mathrm{LD}$ muscle but also the positive and negative $\mathrm{dF} / \mathrm{dt}$. A decline in these parameters during continuous burst stimulation could negatively affect cardiac function when used in dynamic cardiomyoplasty.

Our measurements also demonstrated that increasing the number of bursts/min from 50 burst/min upward decreased force, positive $\mathrm{dF} / \mathrm{dt}$ and negative $\mathrm{dF} / \mathrm{dt}$ development. This holds true for bursts with both $33 \mathrm{msec}$ and $20 \mathrm{msec}$ interpulse intervals. Considering the shift in force-frequency relation after LD muscle transformation, programming of a burst with interpulse intervals of $33 \mathrm{msec}$ appeared sufficient to obtain maximal force. However, our results with bursts with interpulse intervals of $20 \mathrm{msec}$ show that in this way an additional increase in positive $\mathrm{dF} / \mathrm{dt}$ is obtained. In 1965 Buller and Lewis already described that the rate of isometric tension development (16) is enhanced by further increasing the stimulus frequency above that frequency at which maximal skeletal muscle force is obtained. In this way. it seems possible to compensate partially for the loss of power which occurs during the transformation from a fast muscle to a slowly contracting LD muscle (17). In conditioned canine LD muscle we already described the occurrence of greater fatigue using bursts with interpulse intervals shorter than $33 \mathrm{msec}$. However, in that experimental set-up we used bursts with a duration of $250 \mathrm{msec}$ for 100 bursts/min (18). Also Bridges and coworkers described significantly greater fatigue of canine LD muscles using bursts with an interpulse interval of 12 msec compared with an interpulse interval of $40 \mathrm{msec}$ (19). They ascribed these findings to either failure of the excitation-contraction coupling or a reduction in action potential amplitude. If compared to our current goat protocol, They used a longer burst duration . concomitant with a higher number of bursts/min. However, considering the lack of decline in force, positive $\mathrm{dF} / \mathrm{dt}$ and negative $\mathrm{dF} / \mathrm{dt}$ in our goat LD muscles after 10 weeks of stimulation, bursts with an interpulse interval of $20 \mathrm{msec}$ appeared safe. One should be reluctant not to stress the LD muscle in particular at the start of the conditioning protocol. This is underlined by the increase in CK-enzyme level, one day after the first force measurements and the start of electrical stimulation. Most probably damage has been caused by the first fatigue test which appeared to strenuous for the unconditioned LD muscle, considering the pronounced decrease in force during this test. The small rise in CK (Fig 5: VI; S3) at the end of the conditioning period probably can be ascribed to the isometrically measured muscle force during the 1 hour test, in contrast to the more isotonic contraction all animals had apart from these force measurements. The LD muscle damage seen in one goat indicates that not all muscles tolerate the 24 hours presence of electrical stimulation, despite the fact that no continuous elevation of skeletal muscle enzymes was seen. In recent years, pacing protocols of canine and goat $L D$ muscles have been reported using burst stimulation with a maximum of 120 bursts/min (20, 
154
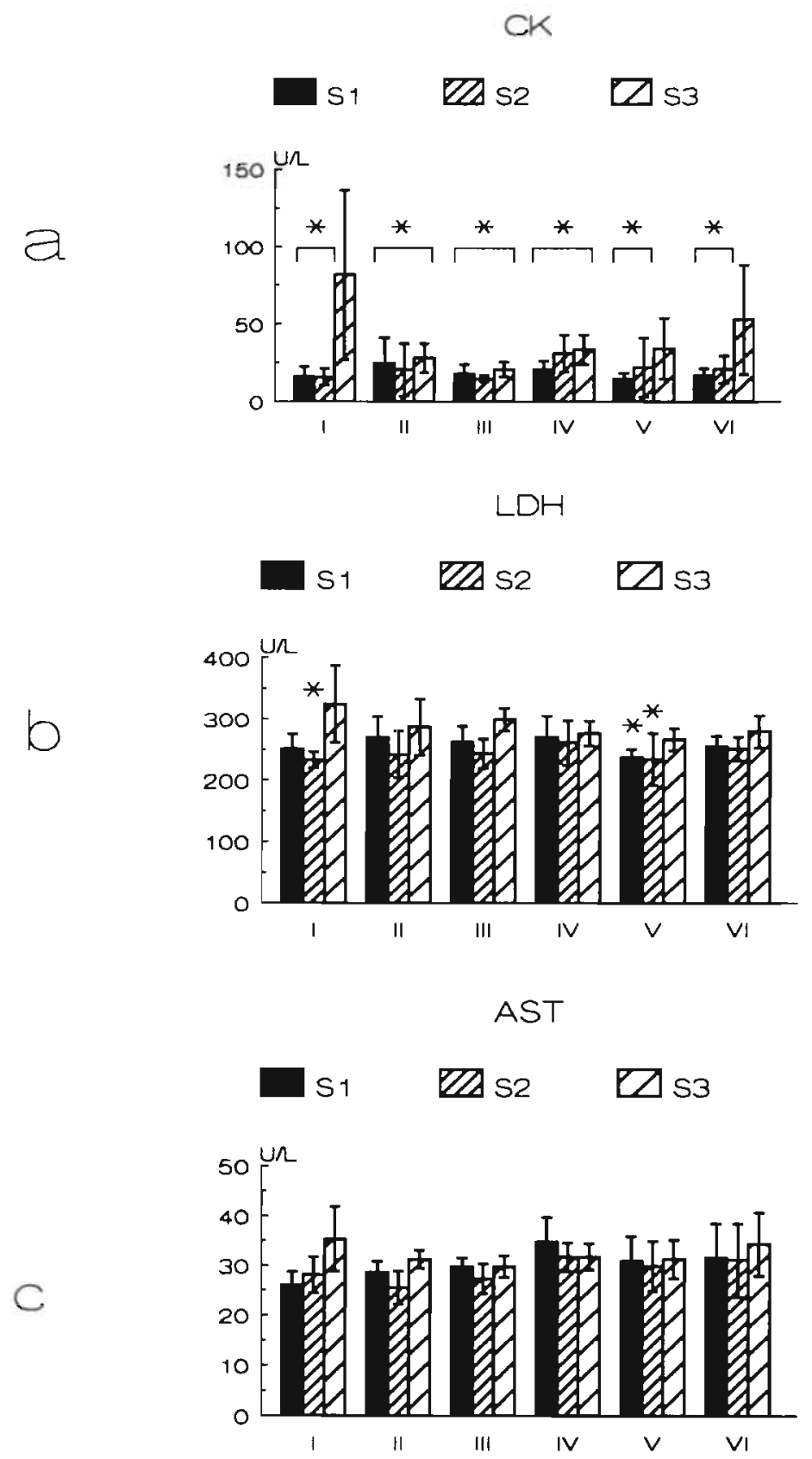

FIGURE 5.

Creatine kinase - (CK) (a), lactate dehydrogenase - (LDH) (b) and aspartate aminotransferase (AST) (b) enzyme-activities, measured at 6 consecutive force measurements done every 2 weeks, (I-VI). S1 means the sample taken just before, S2 the sample immediately after the force measurements. S3 indicates the sample taken 1 day after the measurements. "significantly different from the sample taken 1 day after the first force mecosurements (I;S3), $P<0.05$. 
21). Our data suggest that the upper limit of number of contractions per minute should be below $100 / \mathrm{min}(22$, see Chapter 8$)$. "The exact rate limit probably varies individually and should be based upon measurements of contraction and relaxation speed of the wrapped muscle. It might also be better to use stimulation protocols in which the number of stimulation hours per day is gradually increased. In this way the LD muscle has the opportunity to recover in between the contraction periods at the start of muscle conditioning (21). Furthermore it seems better to search for the lowest possible voltage needed to obtain full recruitment of the stimulated muscle, considering the damage to the tissue surrounding the electrodes. This phenomenon has not been reported in the studies mentioned before, using nerve electrodes with a considerable lower stimulation voltage $(20$, 21).

In conclusion

Application of a stimulation protocol of goat LD muscle, using a short burst with 6 pulses and interpulse intervals of $20 \mathrm{msec}$ during maximally 60 bursts/min, results in a rapid and complete transformation to a fatigueresistant muscle consisting of slowly contracting type I muscle fibres. As long as a limited number of bursts per minute is used, higher positive $\mathrm{dF} / \mathrm{dt}$ can be obtained with bursts with interpulse intervals of $20 \mathrm{msec}$, thereby partially compensating for the increase in contraction- and relaxation time of the transformed LD muscle. 


\section{REFERENCES}

1. Chiu RC-J, Bourgeois IM (Eds.). Transformed muscle for cardiac assist and repair. Futura Publishing Company. Inc. Mount Kisco, NY 1990.

2. Carpentier A, Chachques JC, Grandjean P (Eds.). Cardiomyoplasty. Futura Publishing Company. Inc. Mount Kisco, NY 1991.

3. Stephenson LW (Ed.). J Cardiac Surgery 199 1:6 (suppl).

4. Mayne CN, Anderson WA. Hammond RL, Eisenberg BR. Stephenson LW, Salmons S: Correlates of fatigue resistance in canine skeletal muscle stimulated electrically for up to one year. Am J Physiol 1991;261:C259-C270.

5. Lucas CM. Haventth MG, Van der Veen FH, Habets J, Van der Nagel T, Schrijvers - van Schendel JM, Penn OC. Wellens HJ: Changes in canine latissimus dorsi muscle during 24 weeks of continuous electrical stimulation. J Appl Physiol 1992: 72:828-835.

6. Chachques JC. Grandjean P, Schwartz K. Mihaileanu S. Fardeau M. Swynghedauw B, Fontaliran F. Romero N, Wisnewsky C. Perier P. Chauvaud S. Bourgeois I. Carpentier A: Effect of latissimus dorsi dynamic cardiomyoplasty on ventricular function. Circulation 1988;78 [suppl III):203-216.

7. National Academy of Sciences. Guide for the care and use of laboratory animals. DHHS publication No NIH 85-23, revised 1985.

8. Romanul FCA, Bannister R: Localized areas of high alkaline phosphatase activity in the terminal arterial tree. J Cell Biol 1962;15:73-84.

9. Salmons S. Henriksson J: The adaptive response of skeletal muscle to increased use. Muscle \& Nerve 1991:4:94-105.

10. Levin HR. Tsitlik JE, Halperin HR: Optimization of the timing of skeletal to cardiac muscle contraction during dynamic cardiomyoplasty: Analysis using a mathematical model. J Cardiac Surg 1991;6(suppl):236-244.

11. Salmons S. Jarvis JC: Cardiomyoplasty: A look at the fundamentals. In Carpentier A. Chachques JC. Grandjean P. (Eds). Cardiomyoplasty. Futura Publishing Company Inc.. Mount Kisco, NY 1991:3-17.

12. Salmons $\mathrm{S}$, Jarvis $\mathrm{J}$ : The working capacity of skeletal muscle transformed for use in a cardiac assist role. In: Chiu RC-J. Bourgeois IM (Eds.). Transformed muscle for cardiac assist and repair. Futura Publishing Company Inc., Mount Kisco, NY 1990;89-104.

13. Faulkner JA. Brooks SV: Skeletal muscle fatigue: Implications for circulatoryassist pumps. Sem Thorac Cardiovasc Surg. 1991:3:149-153.

14. De Haan A. Jones DA, Sargeant AJ: Changes in velocity of shortening, power output and relaxation rate during fatigue of rat medial gastrocnemius muscle. Pflügers Arch 1989;413:422-428.

15. Westerblad $\mathrm{H}$, Lannergren $\mathrm{J}$ : Slowing of relaxation during fatigue in single mouse muscle fibres. J Physiol 1991;434:323-336.

16. Buller AJ. Lewis DM: The rate of tension development in isometric tetanic contractions of mammalian fast and slow skeletal muscle. J Physiol 1965: 176:337-354.

17. Brooks SV, Faulkner JA: Forces and powers of slow and fast skeletal muscles in mice during repeated contractions. J Physiol 1991;436:701-710.

18. Lucas CMHB, Van der Veen FH, Grandjean PA, Penn OCKM, Wellens HJJ: What is the ideal pulse frequency for skeletal muscle stimulation after cardiomyoplasty. PACE 1991;14:778-782.

19. Bridges Jr CR, Clark III BJ. Hammond RL, Stephenson LW: Skeletal muscle bioenergetics during frequency-dependent fatigue. Am J Physiol 1991;260: c643-C651. 
20. Chiu RC-J, Walsh GL, Dewar ML, De Simon J-H, Khalafalla AS, Ianuzzo D: Implantable extra-aortic balloon assist powered by transformed fatigue-resistant skeletal muscle. J Thorac Cardiovasc Surg 1987:94:694-701.

21. Kao RL, Trumble DR, Magovern JA, Christlieb IY, Magovern GJ: Fatigue resistant muscle with preserved force and mass for cardiac assist. J Cardiac Surg 1991;6 (suppl):210-217. 


\section{SUMMARY}

Chronic heart failure will be an increasing cause of morbidity and mortality in the coming years. Unfortunately our present methods of treatment are disappointing.

In this thesis a new treatment mode, which is called dynamic cardiomyoplasty, is evaluated. During the procedure a large skeletal muscle, the latissimus dorsi muscle (LD), is used to assist the failing heart. This is done by wrapping the muscle around the heart, followed by electrical stimulation of the muscle synchronous to cardiac contraction. Studies were done in dogs and goats to evaluate the effectiveness of this therapy.

We first of all looked at the changes in the latissimus dorsi muscle that occur during continuous electrical burst stimulation. In Chapter 2 and 3 it is shown that, in both animal species, important changes occur in the muscle, making it more suitable for a continuously active function to assist the heart. A shift was observed from fast-fatiguable type II muscle fibers to fatigue-resistant type I muscle fibers with increased mitochondrial volume and an increase in capillary-to-fibre ratio. This change was accompanied by a decrease in contraction- and relaxation speed, resulting in a decrease in energy costs needed for contraction. Furthermore a decrease in CK- and LDH-activity was noticed. In goats, we also evaluated the increase in connective tissue due to the electrical stimulation.

To be informed about the capacity of the LD muscle to assist a falling heart. a model of acute heart failure was developed, using the tricyclic antidepressive drug imipramine. As shown in Chapter 4, this drug appeared very effective in creating a safe, reversible, well controllable failing heart model without affecting LD muscle force.

In Chapter 5, we demonstrated in acute experiments the capacity of goat LD muscle to increase cardiac pressures and output, using an unconditioned left LD muscle. This was done immediately following counterclockwise wrapping of the muscle around the heart. The importance was shown of stimulating the muscle with a train of pulses to obtain a tetanic contraction. Assistance of the heart was seen both in the normal and failing heart, induced by imipramine.

In Chapter 6, the long-term results are described of 24 goats after a dynamic cardiomyoplasty procedure. As demonstrated, improvement of cardiac function can be obtained as long as the muscle remains vital. The preservation of vitality however, appears to be the main problem. As de- 


\section{SUMMARY}

Chronic heart failure will be an increasing cause of morbidity and mortality in the coming years. Unfortunately our present methods of treatment are disappointing.

In this thesis a new treatment mode, which is called dynamic cardiomyoplasty, is evaluated. During the procedure a large skeletal muscle, the latissimus dorsi muscle (LD), is used to assist the failing heart. This is done by wrapping the muscle around the heart, followed by electrical stimulation of the muscle synchronous to cardiac contraction. Studies were done in dogs and goats to evaluate the effectiveness of this therapy.

We first of all looked at the changes in the latissimus dorsi muscle that occur during continuous electrical burst stimulation. In Chapter 2 and 3 it is shown that, in both animal species, important changes occur in the muscle, making it more suitable for a continuously active function to assist the heart. A shift was observed from fast-fatiguable type II muscle fibers to fatigue-resistant type I muscle fibers with increased mitochondrial volume and an increase in capillary-to-fibre ratio. This change was accompanied by a decrease in contraction- and relaxation speed, resulting in a decrease in energy costs needed for contraction. Furthermore a decrease in CK- and LDH-activity was noticed. In goats, we also evaluated the increase in connective tissue due to the electrical stimulation.

To be informed about the capacity of the LD muscle to assist a falling heart. a model of acute heart failure was developed, using the tricyclic antidepressive drug imipramine. As shown in Chapter 4, this drug appeared very effective in creating a safe, reversible, well controllable failing heart model without affecting LD muscle force.

In Chapter 5, we demonstrated in acute experiments the capacity of goat LD muscle to increase cardiac pressures and output, using an unconditioned left LD muscle. This was done immediately following counterclockwise wrapping of the muscle around the heart. The importance was shown of stimulating the muscle with a train of pulses to obtain a tetanic contraction. Assistance of the heart was seen both in the normal and failing heart, induced by imipramine.

In Chapter 6, the long-term results are described of 24 goats after a dynamic cardiomyoplasty procedure. As demonstrated, improvement of cardiac function can be obtained as long as the muscle remains vital. The preservation of vitality however, appears to be the main problem. As de- 
160

monstrated, destruction of skeletal muscle cells and replacement by fatty cells occurs after a certain period of electrical stimulation.

We can only speculate about possible causes for the loss of inuscle fibre vitality like: 1) Marked changes in the original orientation of the muscle fibers: 2) Insufficient arterial blood supply as demonstrated by the absence of an increase in capillary-to-fibre ratio, in contrast to the in situ stimulated LD muscle; 3) A more pronounced increase in connective tissue; 4) Destruction of nerve branches; 5) The use of a stimulation protocol which is to strenuous for the LD muscle.

Different ways of stimulating the conditioned, transformed LD muscle are evaluated in in situ stimulated canine LD muscles in Chapter 7. Using 100 contractions/minute, we tested bursts of electrical pulses using different interpulse intervals. An interval of $33 \mathrm{msec}$ appeared to result in the most optimal preservation of force.

Data presented in Chapter 8 demonstrate the danger of pacing the latis simus dorsi muscle with a protocol, which appears to strenuous for this muscle. In that chapter we demonstrated, both in goats and in two patients, that the currently clinically used stimulation protocol may have a long-term detrimental effect on LD muscle function. Using 100 bursts/minute, consisting of bursts of either $185 \mathrm{msec}$ or 240 msec burst duration with interpulse intervals of $33 \mathrm{msec}$, resulted particularly in insufficient relaxation of the LD muscle. Insufficient relaxation probably leads to chronic skeletal muscle ischemia and loss of function.

Thereíre, as described in Chapter 9, we tested in the goat a more prudent stimulation protocol. As discussed, this was evaluated using the in situ stimulated LD muscle. Pacing was performed using a shorter burst duration and a maximum number of burst contractions of $60 /$ minute. This protocol induced changes in contraction characteristics during the transformation process of the LD muscle which appeared to be more favorable.

This protocol has to be tested in experiments with long-term wrapped LD muscles.

\section{In conclusion}

Our findings indicate that, by using electrical stimulation, a fast-twitch muscle can change its properties and behave as a slow-twitch muscle, which is in line with from other investigators. When wrapped around the heart such a muscle can support and improve cardiac pump function.

A major problem, which still has to be solved, is the identification of the best stimulation program for the wrapped muscle. First of all, the goal is to have optimal conditioning and performance of the muscle in time and, secondly, a long-term pacing protocol allowing optimal contraction and relaxation of the wrapped muscle without the induction of degenerative changes. 


\section{SAMENVATTING}

Daling van de sterfte aan het hartinfarct en toenemende vergrijzing van de bevolking maken dat in de komende jaren chronisch hartfalen een steeds belangrijkere oorzaak zal worden van chronische ziekte en sterfte. Helaas zijn de huidige medicamenteuze behandelingsmogelijkheden teleurstellend en andere behandelingsvormen als harttransplantatie schaars en duur.

In dit proefschrift worden de ervaringen beschreven met een nieuwe vorm van therapie, geheten de dynamische cardiomyoplastie. Hierbij wordt een van de schouderspieren, de linker latissimus dorsi, in de borstkas gebracht en om het hart gewikkeld. Vervolgens wordt deze tot samentrekking gebracht, tegelijk met het hart, door de spier te stimuleren met electrische pulsen. Deze nieuwe methodiek werd bestudeerd zowel bij honden als geiten. In Hoofdstuk 2 worden de veranderingen beschreven die optreden in de latissimus dorsi spier (LD) van de hond, wanneer deze, ter plaatse, geduren= de een periode van maximaal 24 weken, voortdurend electrisch was geprikkeld. Hierbij werd gevonden dat de spier zich aanpast aan de continue arbeid door de contractie- en relaxatiesnelheid van de spier te verminderen, waardoor de benodigde energie afneemt. Daarnaast is er een toename in de verhouding bloedvaatjes/spiervezels, een toename in het mitochondriaal volume en een transformatie van de snel vermoeibare type II spiervezel naar de vermoeidheidsresistente type I vezel. Ook was er een afname in glycolytische capaciteit zichtbaar.

In Hoofdstuk 3 wordt beschreven dat deze veranderingen ook optreden bij de latissimus dorsi spier van de geit. Daarnaast hebben wij bij de geit ook een duidelijke toename van bindweefsel in de spierbundels gezien.

Om een juist beeld te verkrijgen omtrent de mogelijkheden orn met behulp van de gewikkelde latissimus dorsi een falend hart te ondersteunen, hebben wij gebruik gemaakt van een falend hartmodel wat veroorzaakt werd door het tricyclische antidepressivum imipramine. Dit wordt beschreven in Hoofdstuk 4. Hierin wordt aangetoond dat het met imipramine mogelijk is snel reversibel hartfalen te induceren zonder dat het proefdier overlijdt en zonder negatief effect op de skeletspierkracht.

In Hoofdstuk 5 wordt de chirurgische techniek van het wikkelen van de spier beschreven. Tevens wordt aangetoond dat de ongetrainde spier, onmiddelijk na het wikkelen, in staat is de functie van zowel het normale als het falende hart te verbeteren. Daarbij blijkt dat een reeks van electrische prikkels, een zgn. burst, die leidt tot een tetanische contractie van de 


\section{2}

gewikkelde spier, effectiever is dan enkele pulsen.

In Hoofdstuk 6 worden de ervaringen beschreven met 24 geiten waarbij gedurende vele: weken de cardiomyoplastie methodiek was toegepast. Het bleek dat inderdaad verbetering van de cardiale functie mogelijk was, indien de gewikkelde en gestimuleerde musculus latissimus dorsi vitaal blijft. Dit laatste is echter een groot probleem. Vaak werd gevonden dat delen van het skeletspierweefsel verloren waren geraakt met aansluitend vervanging door vetweefsel. Mogelijke oorzaken voor dit spierverlies zijn; 1] Belangrijke veranderingen in de vezelrichting van de musculus latissimus dorsi na het wikkelen; 2) Het uitblijven van een toename in capillair/vezel ratio in tegenstelling tot ter plaatse gestimuleerde spieren; 3) Beschadiging van zenuwtakken; 4) Toename van bindweefsel in de gestimuleerde spier; 5) Het gebruiken van een mogelijk te intensief stimulatieprotocol van de gewikkelde spier.

De beste manier van electrisch prikkelen van de geconditioneerde latissimus dorsi spier is een probleem omdat er belangrijke veranderingen in mechanische karakteristieken van de latissimus dorsi spier plaatsvinden tijdens continue stimulatie. In Hoofdstuk 7 wordt beschreven dat een stimulatieprogramma wat gebruik maakt van 100 burst-contracties/minuut en een interpulse interval van 33 msec leidt tot het beste behoud van de spierkracht. Bewijs voor een mogelijk te intensief stimulatieprotocol, zoals dat tot nu toe toegepast werd, wordt geleverd in Hoofdstuk 8. Hierin wordt, zowel bij geiten als bij patiënten, aangetoond dat bij het stimuleren van de spier met 100 burstcontracties/minuut, met een burstduur van zowel 185 als $240 \mathrm{msec}$, onvoldoende relaxatie van de spier optreedt. Dit zou kunnen leiden tot ischemie van de spier en verlies van spierfunctie.

Vandaar dat in Hoofdstuk 9 een eerste aanzet is gegeven tot het gebruik van een nieuw stimulatieprotocol voor de ter plaatse geconditioneerde latissimus dorsi spier, waarbij een kortere burstduur wordt gebruikt met een maximum van 60 burstcontracties/minuut. Het bleek dat hiermee de negatieve effecten van de transformatie van de spier, d.w.z. afname in contractie- en relaxatiesnelheid grotendeels kunnen worden opgevangen. Dit protocol zal in de toekomst verder moeten worden uitgetest bij de chronisch gewikkelde latissimus dorsi spier.

Simmenvattend laat ons onderzoek, evenals dat van anderen, zien dat het goed mogelijk is om met behulp van electrische prikkeling de eigenschappen van een iich snel samentrekkende spier te veranderen in die van een zich langzamer samentrekkende onvermoeibare spier. Wanneer dan de spier rond het hart wordt gewikkeld is zij in staat de hartfunktie te ondersteunen en te verbeteren. Een groot problecm, wat nog niet is opgelost, blijft het vinden van het beste stimulatieprogramma voor de wikkelspier. Het uiteindelijke doel is tweeërlei. Ten eerste het bereiken van een zo goed mogelijke en snelle verandering in spiereigenschappen van de wikkelspier, zodat chronisch werkzaamheden mogelijk worden zonder vermoeidheidsverschijnselen. Ten tweede moet het prikkelprogramma zodanig zijn, dat de wikkelspier tijdens chronische arbeid voldoende tijd krijgt voor optimale spiersamentrekking en spierontspanning, en dat er geen degeneratieve veranderingen optreden. 


\section{PUBLICATIONS}

\section{ABSTRACTS AND PROCEEDINGS}

$\infty$ 'Monitoring of muscle fiber type of canine Latissimus Dorsi muscle during chronic electrical stimulation by enzyme- and immunohistochemistry'. MG Havenith, FH van der Veen, JFC Glatz, CMHB Lucas, JMC Schrijvers- van Schendel. OCKM Penn, HJJ Wellens.

In: Proceedings of Third International symposium on transformed skeletal muscle for cardiac assist and repair. 1988; abstract 4.

\% In vivo contraction analysis as a measure of Latissimus Dorsi muscle adaptation following chronic electrical stimulation:. FH van der Veen, MG Dassen. MG Havenith, C Lucas, Th van der Nagell, OCKM Penn, HJJ Wellens.

In: Proceedings of Third International symposium on transformed muscle for cardiac assist and repair. 1988;abstract 9.

'The acute effect of L-Carnitine on skeletal muscle fatiguetests in the dog'. CMHB Lucas, FH van der Veen, M-L Dubelaar, C Koks, T van der Nagel, WC Hülsmann, HJJ Wellens.

In: Proccedings of 2 nd International Symposium on Lipid Metabolism in the Normoxic and Ischemic Heart. 1988;50.

$\infty$ 'Canine Latissimus Dorsi muscle stimulation after transformation'. CMHB Lucas. MG Havenith, OCKM Penn, FH van der Veen, HJJ Wellens.

J Physiol 1989:C98.

$\hookrightarrow$ 'Evidence of Latissimus Dorsi muscle adaptation before cardiomyoplasty'. FH van der Veen, MG Havenith. P Grandjean, CMHB Lucas, W Dassen. JACC 1989;13(2):214A.

$\leftrightarrow$ 'Pulse frequency selection for skeletal muscle stimulation'. FH van der Veen. CMHB Lucas, P Grandjean, J Habets, T van der Nagel, OCKM Penn, HJJ Wellens.

In: Proceedings of First International Workshop on Cardiomyoplasty. 1989; 5. 


\section{4}

$\infty$ 'Effect of farmacological agents on the trained Latissimus Dorsi muscle'. MJW Smets, CMHB Lucas, FH van der Veen, OCKM Penn, S de Lange, HJJ Wellens.

In: Proceedings of First International Workshop on Cardiomyoplasty. 1989; 14.

$\rightarrow$ 'Assistance of an acute failing heart with the left Latissimus Dorsi muscle in dogs'. CMHB Lucas, OCKM Penn, FH van der Veen, J Habets, T van der Nagel, B Terpstra, MJW Smets, HJJ Wellens.

In: Proceedings of First International Workshop on Cardiomyoplasty. 1989; 20.

$\Leftrightarrow$ 'Cardiac assist with the left Latissimus Dorsi muscle in dogs'. OCKM Penn, CMHB Lucas, FH van der Veen, J Habets, T van der Nagel, B Terpstra, MJW Smets, HJJ Wellens.

In: Proceedings of the 3rd Annual meeting of The Europian Association for Cardio-thoracic Surgery. 1989.

$\infty$ 'A reversible failing heart model in dogs by infusion of imipramine'. C Lucas, F van der Veen, E Cheriex, J Habets, T van der Nagel. H Wellens, F Prinzen. The FASEB Journal 1990;4(3):A757.

$\rightarrow$ 'Conditioning of canine Latissimus Dorsi muscle during 24 weeks before cardiomyoplasty'. C Lucas, F van der Veen, M Havenith. J Habets, T van der Nagel, H Wellens.

PACE 1990; 13:531.

$\Leftrightarrow$ 'Cardiomyoplasty improves cardiac function in the failing heart of the dog'. C Lucas, O Penn, F van der Veen, J Habets, T van der Nagel, B Terpstra, H Wellens.

PACE 1990;13:555.

$\infty$ 'Improvement of cardiac function after cardiomyoplasty in the dog'. FH van der Veen. C Lucas. OCKM Penn, HJJ Wellens. J Mol Cell Cardiol 22 (suppl III) $1990 ; 90$.

$\omega$ 'The acute effect of L-Carnitine on skeletal muscle force tests in the dog'. M-L Dubelaar, CMHB Lucas, FH van der Veen, WC Hülsmann. Pflügers Arch 1990:416;3.

observations during early muscle stimulation following dynamic cardiomyoplasty.' R Lorusso, C Lucas, F van der Veen, T van der Nagel, J Habets. M-L Dubelaar, H Wellens, O Penn. PACE 1991; 14(4):669.

$\leftrightarrow$ 'A comparison of two conditioning protocols on goat latissimus dorsi muscle'. C Lucas, M-L Dubelaar, F van der Veen, B Terpstra, H Wellens. PACE 1991;14(4):691. 
$\Leftrightarrow$ 'Optimal stimulation of the goat latissimus dorsi muscle using the 'Prometheus' pacemaker system'. C Lucas, M-L Dubelaar, F van der Veen, B Terpstra, H Wellens.

PACE 1991;14(4):735.

$\Leftrightarrow$ 'Selecting stimulus strength after cardiomyoplasty'.

FH van der Veen, C Lucas, R Lorusso, T van der Nagel, OC Penn, HJJ Wellens.

JACC 1991;17(2):272A.

$\hookrightarrow$ 'Successful anticipated latissimus dorsi muscle stimulation following dynamic cardiomyoplasty procedure'. R Lorusso, C Lucas, FH van der Veen, $\mathrm{T}$ van der Nagel, M Havenith, OC Penn. Eur Heart $J$ 1991: 12(suppl);abstract. 2030.

$\infty$ 'The dog as a model for skeletal muscle assist'. FH van der Veen, C Lucas, OCKM Penn, HJJ Wellens. In: Harnessing skeletal muscle power for cardiac assistance. Eds: Salmons and Jarvis; Proceedings of the expert meeting, Liverpool, 1991.

$\leftrightarrow$ Evaluation of a prudent conditioning protocol of the latissimus dorsi muscle in goats'. C Lucas, M Dubelaar, $F$ van der Veen, $J$ Habets, $T$ van der Nagel, H Wellens. PACE 1992: 15;561.

\section{CHAPTERS IN BOOKS}

$\Leftrightarrow$ 'Monitoring of muscle fiber type of canine Latissimus Dorsi muscle during chronic electrical stimulation by enzyme- and immunohistochemistry'. MG Havenith, FH van der Veen, JFC Glatz, CMHB Lucas, JMC Schrijvers- van Schendel, OCKM Penn, HJJ Wellens.

In: Transformed muscle for cardiac assist and repair. Chiu RC-J, Bourgeols IM (Eds.), Futura Publishing Company Inc., Mount Kisco, NY 1990;53-61.

$\leftrightarrow$ 'In vivo contraction analysis as a measure of Latissimus Dorsi muscle adaptation following chronic electrical stimulation'. Van der Veen FH, Dassen WRM, Havenith MG. Lucas C, Van der Nagel Th, Penn OCKM, Wellens HJJ.

In: Transformed muscle for cardiac assist and repair. Chiu RC-J, Bourgeois IM (Eds.). Futura Publishing Company Inc., Mount Kisco, NY 1990; 105115.

$\leadsto$ 'Effect of farmacological agents on the trained Latissimus Dorsi muscle'. MJW Smets, CMHB Lucas, FH van der Veen, OCKM Penn, S de Lange, HJJ Wellens.

In: Cardiomyoplasty. Carpentier A, Chachques JC, Grandjean P (Eds.), Futura Publishing Company Inc., Mount Kisco, NY 1991;209-215. 


\section{6}

$\hookrightarrow$ 'Early cardiac assist following dynamic cardiomyoplasty: An experimental study'. R Lorusso, C Lucas, F van der Veen, T van der Nagel, J Habets, H Wellens, O Penn.

In: Heart Surgery 1991; 4th International symposium on cardiac surgery. D'Allesandro LC (Ed.). Casa Editrice Scientifica Internationale 1991;573578.

$\hookrightarrow$ 'Fibrin sealant application during dynamic cardiomyoplasty'. R Lorusso, C Lucas, F van der Veen. J Habets, T van der Nagel, M Havenith, HJ Wellens, OC Penn.

In: Heart Surgery 1991; 4th International symposium on cardiac surgery. D'Allesandro LC (ed.). Casa Editrice Scientifica Internationale 1991;579584.

\section{FULL PAPERS}

$\rightarrow$ 'Het Wikkelhart: een nieuw alternatief bij chronisch hartfalen'. GVA van Ommen. OCKM Penn, JBRM de Swart, MJW Smets, CMHB Lucas, HJJ Wellens.

Ned Tijdschr Geneesk 1990;134:1748-1750.

What is the ideal pulse frequency for skeletal muscle stimulation after cardiomyoplasty'. CMHB Lucas, FH Van der Veen, PA Grandjean, OCKM Penn, HJJ Wellens. PACE 1991;14:778-782.

c) 'Acute effect of L-Carnitine on skeletal muscle force tests in dogs'. M-L Dubelaar, CMHB Lucas, WC Hülsmann.

Am J Physiol 1991:260:E189-E193.

$\hookrightarrow$ 'A new method to select stimulus strength after cardiomyoplasty' FH van der Veen, C Lucas, R Lorusso, T van der Nagel, OCKM Penn, HJJ Wellens. J Cardiac Surg 1991:6(1)suppl:259-264.

$\infty$ 'Canine-specific adaptation of energy metabolism of Latissimus Dorsi muscle in response to chronic electrical stimulation'. JFC Glatz, GJ van der Vusse, MG Havenith, FH van der Veen, CMHB Lucas, OCKM Penn, HJJ Wellens.

J Cardiac Surg 1991:6(1)suppl:265-269.

$\rightarrow$ The effect of L-Carnitine on force development of the Latissimus Dorsi muscle in dogs'. M-L Dubelaar, CMHB Lucas. WC Hulsmann. J Cardiac Surg 1991;6(1):270-275.

$\infty$ 'Early latissimus dorsi stimulation after cardiomyoplasty procedure: A preliminary study'. R Lorusso, C Lucas, F van der Veen, J Habets, T van der Nagel, M Havenith, HJ Wellens, OC Penn.

Basic Appl Myol 1991;1(1):83-87. 
$\infty$ 'On the mechanism of fat accumulation in wrapped latissimus dorsi muscle (cardiomyoplasty) and the effect of chronic L-carnitine administration'. Basic Appl Myol 1991;1:305-310.

$\Leftrightarrow$ Adaptation of energy metabolism of canine latissimus dorsi muscle in response to chronic electrical stimulation'. JFC Glatz, GJ van der Vusse, MG Havenith, FH van der Veen, CMHB Lucas, OCKM Penn, HJJ Wellens. Pflügers Arch 1992;420:1-8.

$\rightarrow$ 'Changes in canine latissimus dorsi muscle during 24 weeks of continuous electrical stimulation'. CM Lucas, MG Havenith, FH van der Veen, J Habets, T van der Nagel, LM Schrijvers - van Schendel, OC Penn, HJ Wellens. J Appl Physiol 1992;72:828-835.

$\Leftrightarrow$ Imipramine induced heart failure in the dog: A model to study the effect of cardiac assist devices'. CMHB Lucas, EC Cheriex, FH van der Veen, J Habets, $T$ van der Nagel, OC Penn, HJJ Wellens. Cardiovasc Res. In press.

$\Leftrightarrow$ 'The importance of muscle relaxation in dynamic cardiomyoplasty'. CM Lucas, $F$ van der Veen. EC Cheriex. V van Ommen, OC Penn, HJ Wellens. PACE. In press.

$\Leftrightarrow$ 'Differences in metabolic response of dog and goat latissimus dorsi muscle to chronic electrical stimulation'. JFC Glatz, YF de Jong, WA Coumans, CM Lucas. FH van der Veen, GJ van der Vusse. Am J Physiol. In press.

$\hookrightarrow$ 'Hemodynamic study following cardiomyoplasty: Comparison of different stimulation protocols'. CM Lucas, RC Lorusso, ML Dubelaar, FH van der Veen, MA Vos, J Habets. Th van der Nagel, OC Penn, HJ Wellens. Presented for publication.

$\Leftrightarrow$ 'Adaptations of goat latissimus dorsi muscle using two different protocols of continuous electrical stimulation'. CM Lucas, M-L Dubelaar, FH van der Veen, EN Kloostemnan - Castro-Ravelo, MG Havenith, J Habets, Th van der Nagel, OC Penn, HJ Wellens. Presented for publication.

$\leftrightarrow$ 'Long-term follow-up after dynamic cardiomyoplasty in goats'. CMHB Lucas. FH van der Veen, EC Cheriex, R Lorusso, M Havenith, OCKM Penn, HJJ Wellens. Presented for publication.

$\Leftrightarrow$ 'A new stimulation protocol for cardiac assist using the latissimus dorst muscle'. CM Lucas, ML Dubelaar, FH van der Veen, E Kloosterman Castro-Ravelo, M Havenith, J Habets, Theo van der Nagel, OC Penn, HJ Wellens. Presented for publication. 


\section{NAWOORD}

Een groot aantal mensen hebben mij gedurende de afgelopen jaren bijgestaan, zowel in het kader van het onderzoek als ook daarbuiten.

Allereerst wil ik Prof. Dr. Hein Wellens bedanken voor zijn functie als coördinator en sturende kracht binnen dit onderzoek. Helaas heeft dit onderzoek op dit moment nog niet geresulteerd in effectieve klinische toepassing van deze therapie ter behandeling van chronisch hartfalen. Echter, na verder onderzoek o.a. naar optimale latissimus dorsi stimulatie zal mogelijk deze methodiek toch op uitgebreidere schaal zijn ingang vinden binnen de afdeling Cardiologie/Cardiochirurgie Maastricht.

Dr. Ir. Erik van der Veen was (mede)-verantwoordelijk voor de eerste opzet van dit onderzoek. Daarnaast fungeerde hij als begeleider en coördinator van de praktische aspecten van dit onderzoek. Hiervoor ben ik hem zeer erkentelijk.

Gaarne wil ik Prof. Dr. Olaf Penn bedanken voor de uurtjes die hij wist vrij te maken voor het 'wikkelen' van de honden en de geiten. Voor overname van een deel van dit werk was Drs. Roberto Lorusso verantwoordelijk.

De leden van de beoordelingscommissie: Prof. Dr. R.S. Reneman (voorzitter), Prof. Dr. G. Elzinga, Prof. Dr. W Hülsmann. Prof. Dr. J.W. Arends en Prof. Dr. S. Salmons wil ik bedanken voor de tijd en energie gestoken in het beoordelen van het manuscript.

Drs. Emile Cheriex wist steeds weer een gaatje te vinden in zijn druk bezette programma om Echo-Dopplermetingen bij de proefdieren te komen doen.

De afdeling Pathologie heeft eveneens een belangrijke rol gespeeld in het verkrijgen van resultaten binnen dit onderzoek. Dr. Miek Havenith als hoofdverantwoordelijke voor het bekijken en beoordelen van de histologische preparaten. Daamaast Drs. Rob Visser die deze taak tijdelijk van hem heeft overgenomen. Mevrouw Anita Schrijvers-van Schendel en de heer Ruud Aarts zorgden voor het vervaardigen van de histologische coupes en mevrouw Judith Stouten voor het prepareren van de electronenmicroscopische foto's. Tevens wil ik de mensen op het laboratorium Pathologie bedanken voor de gezelligheid en de hulp o.a. wanneer er weer eens een extra pot formaline versierd moest worden voor een wikkelhart. Mevrouw Marie-Louise MullersBoumans wil ik bedanken voor het doen van de enzymatische bepalingen. Binnen de vakgroep Fysiologie zijn een aantal mensen die mij met raad en daad hebben bijgestaan. Hiervan wil ik noemen Dr. Jan Glatz. Dr. Theo Arts 
en Dr. Harm Kuipers. Mevrouw Jet Beekman, de heer Theo van der Nagel en de heer Ruud Kruger waren medeverantwoordelijk, niet alleen voor de gang van zaken tijdens de dierexperimenten, maar ook voor de altijd aanwezige koffie en kletskoek in de vroege ochtenduren. Van de mensen van de Centrale Proefdier Voorziening wil ik de de heer Peter Franssen en de heer Frans Slangen bedanken voor de verzorging van de proefdieren en Drs. Ton van den Boogaard voor zijn supervisie over dit geheel. De heer Rob Mulleneers en de heer Berto Bleylevens hebben gezorgd voor het oplossen van vele computertechnische 'plaatjes' problemen. De heer Ruud Leliveld is verantwoordelijk voor het vervaardigen van de lay-out en het drukken van dit proefschrift.

Op 'pacemakergebied' heb ik nooit tevergeefs om hulp gevraagd van de heren Pierre-Andre Grandjean, Ben Terpstra, Francois Chaussende en Chester Struble van de firma Medtronic [Bakken Research Center Maastricht]. Deze firma leverde tevens het benodigde electrische materiaal voor de experimenten.

Tenslotte wil ik nog een aantal mensen noemen die een belangrijke en beslissende rol hebben gespeeld in dit onderzoek. Drs. Jan van Oppen vanwege de hulp bij vele kleine en grote praktische zaken en vanwege de inspirerende 'debatten' over onderzoek in het algemeen. Dr. Marc Vos. die me op het juiste moment uit het beruchte onderzoeksdal heeft getrokken en me gewezen heeft op de top die komt. Mevrouw Elisa Kloosterman CastroRavelo, die met niet-aflatende inzet tot in de avonduren honderden histologische coupes heeft vervaardigd en bekeken. Een belangrijk deel van het beschreven onderzoek is op deze data gebaseerd. De heer Jo Habets, die niet alleen grotendeels verantwoordelijk was voor de assistentie bij de experimenten en de nauwkeurige zorg voor de proefdieren, maar me ook een inspirerende woon- en werkomgeving verschaft heeft in het hoogste kerkdorp van Nederland. Marie-Louise Dubelaar, met wie ik een groot deel van de praktische uitvoering en verwerking van het onderzoek gedaan heb (naast de dagelijkse boodschappen bij AH). Mede daarom ben ik verheugd dat het toch gelukt is onze promoties op dezelfde dag te plannen.

Tenslotte wil ik mijn ouders bedanken op wie ik nooit tevergeefs een beroep heb gedaan en die me ten alle tijden in woord en daad terzijde hebben gestaan. 


\section{CURRICULUM VITAE}

1960

Geboren op 27 september te Etten-Leur.

1960-1978

Opgegroeid te Etten-Leur.

$1972-1978$

Gymnasium $\beta$ aan het Norbertuscollege te Roosendaal.

1978-1979

Studie aan de Interfakulteit Lichamelijke Opvoeding, Vrije Universiteit te Amsterdam.

1979-1987

Studie Geneeskunde aan de Vrije Universiteit te Amsterdam.

1988-januari 1992

Toegevoegd onderzoekster binnen het project 'Cardiomyoplastie', uitgevoerd onder leiding van Prof. Dr. HJJ Wellens, afdeling Cardiologie, Academisch Ziekenhuis Maastricht, Rijksuniversiteit Limburg te Maastricht.

v.a. januari 1992

Werkzaam als arts-assistente op de afdeling Cardiologie in het Academisch Ziekenhuis Maastricht. 
